\title{
Cleanup Verification Package for the 100-K-55:1 and 100-K-56:1 Pipelines and the 116-KW-4 and 116-KE-5 Heat Recovery Stations
}

\author{
Prepared for the U.S. Department of Energy \\ by Washington Closure Hanford
}




\section{EXECUTIVE SUMMARY}

This cleanup verification package documents completion of remedial action for the 100-K-55:1 and 100-K-56:1 reactor cooling effluent underground pipelines, referred to herein as the 100-K-55:1 and 100-K-56:1 sites, as well as for the 116-KW-4 and 116-KE-5 heat recovery stations, referred to herein as the 116-KW-4 and 116-KE-5 sites. The 116-KW-4 and 116-KE-5 heat recovery stations were co-located and remediated with the 100-K-55:1 and 100-K-56:1 pipelines, respectively. These sites are located in the 100-KR-2 Operable Unit in the 100-K Area of the Hanford Site in southeastern Washington State. The 100-K-55 and 100-K-56 sites consisted of those process effluent pipelines that serviced the 105-KW and 105-KE Reactors, respectively. Both of these sites have been administratively divided into subunits based on the current extent of remediation. Portions of the pipelines remaining within the reactor security fencing and in proximity to active utility features have been delineated as the 100-K-55:2 and 100-K-56:2 pipelines, with the portions of the pipelines excluded from these boundaries identified as the 100-K-55:1 and 100-K-56:1 pipelines. This cleanup verification package addresses only the 100-K-55:1 and 100-K-56:1 subunits; the 100-K-55:2 and 100-K-56:2 subunits will be addressed within a separate cleanup verification package.

Site excavation and waste disposal are complete, and the exposed surfaces have been sampled and analyzed to verify attainment of the remedial action goals. Results of the sampling, laboratory analyses, and data evaluations for the 100-K-55:1, 100-K-56:1, 116-KW-4, and 116-KE-5 sites indicate that all remedial action objectives and goals for direct exposure, protection of groundwater, and protection of the Columbia River have been met (see Table ES-1).

The sites meet cleanup standards and have been reclassified as "interim closed out" in accordance with the Hanford Federal Facility Agreement and Consent Order (Ecology et al. 1989) and the Waste Site Reclassification Guideline TPA-MP-14 (RL-TPA-90-0001) (DOE-RL 1998). Copies of the waste site reclassification forms for each site are included as Attachments ES-1 through ES-4. 


\section{Table ES-1. Summary of Cleanup Verification Results for the 100-K-55:1, 100-K-56:1, 116-KW-4, and 116-KE-5 Sites. (2 Pages)}

\begin{tabular}{|c|c|c|c|c|}
\hline $\begin{array}{l}\text { Regulatory } \\
\text { Requirement }\end{array}$ & Remedial Action Goals & Results & $\begin{array}{l}\text { Remedial } \\
\text { Action } \\
\text { Objectives } \\
\text { Attained? }\end{array}$ & Ref. \\
\hline $\begin{array}{l}\text { Direct Exposure - } \\
\text { Radionuclides }\end{array}$ & $\begin{array}{l}\text { 1. Attain } 15 \mathrm{mrem} / \mathrm{yr} \text { dose rate } \\
\text { above background over } \\
1,000 \text { years. }\end{array}$ & $\begin{array}{l}\text { 1. Maximum dose rate calculated by } \\
\text { RESRAD for the } 100-\mathrm{K}-55: 1 \text { site is } \\
4.59 \mathrm{mrem} / \mathrm{yr} \text { (not accounting for } \\
\text { backfill). Maximum dose rate } \\
\text { calculated by RESRAD for the } \\
100-\mathrm{K}-56: 1 \text { site is } 9.95 \mathrm{mrem} / \mathrm{yr} \text { (not } \\
\text { accounting for backfill). No } \\
\text { radionuclide COCs were identified } \\
\text { for the } 116-\mathrm{KW}-4 \text { or } 116-\mathrm{KE}-5 \text { sites. }\end{array}$ & Yes & $a, b$ \\
\hline $\begin{array}{l}\text { Direct Exposure - } \\
\text { Nonradionuclides }\end{array}$ & 1. Attain individual COC RAGs. & $\begin{array}{l}\text { 1. No nonradionuclide COCs were } \\
\text { identified for the 100-K-55:1 or } \\
100-\mathrm{K}-56: 1 \text { sites. Individual } \\
\text { concentrations of COPCs are below } \\
\text { the RAGs at the } 116-\mathrm{KW}-4 \text { and } \\
116-\mathrm{KE}-5 \text { sites. }\end{array}$ & Yes & \\
\hline \multirow[t]{4}{*}{$\begin{array}{l}\text { Meet } \\
\text { Nonradionuclide Risk } \\
\text { Requirements }\end{array}$} & $\begin{array}{l}\text { 1. Hazard quotient of }<1 \text { for } \\
\text { noncarcinogens. }\end{array}$ & $\begin{array}{l}\text { 1. No nonradionuclide COCs were } \\
\text { identified for the } 100-\mathrm{K}-55: 1 \text { and } \\
100-\mathrm{K}-56: 1 \text { sites. Individual hazard } \\
\text { quotient values for the } 116-\mathrm{KW}-4 \\
\text { and } 116-\mathrm{KE}-5 \text { sites are less than } 1 .\end{array}$ & \multirow{4}{*}{ Yes } & c \\
\hline & $\begin{array}{l}\text { 2. Cumulative hazard quotient of } \\
<1 \text { for noncarcinogens. }\end{array}$ & $\begin{array}{l}\text { 2. No nonradionuclide COCs were } \\
\text { identified for the } 100-\mathrm{K}-55: 1 \text { and } \\
\text { 100-K-56:1 sites. Cumulative } \\
\text { hazard quotients for the } 116-\mathrm{KW}-4 \\
\text { and } 116-\mathrm{KE}-5 \text { sites are } 1.0 \times 10^{-3} \\
\text { and } 3.4 \times 10^{-2} \text {, respectively. }\end{array}$ & & c \\
\hline & $\begin{array}{l}\text { 3. Excess cancer risk of }<1 \times 10^{-6} \text { for } \\
\text { individual carcinogens. }\end{array}$ & $\begin{array}{l}\text { 3. No nonradionuclide COCs were } \\
\text { identified for the } 100-\mathrm{K}-55: 1 \text { and } \\
100-\mathrm{K}-56: 1 \text { sites. Excess cancer } \\
\text { risk values for residual hexavalent } \\
\text { chromium (the sole carcinogenic } \\
\mathrm{COPC} \text { ) at the } 116-\mathrm{KW}-4 \text { and } \\
116-\mathrm{KE}-5 \text { sites are } 1.1 \times 10^{-7} \text { and } \\
1.6 \times 10^{-7} \text {, respectively. }\end{array}$ & & c \\
\hline & $\begin{array}{l}\text { 4. Attain a total excess cancer risk of } \\
<1 \times 10^{-5} \text { for carcinogens. }\end{array}$ & $\begin{array}{l}\text { 4. No nonradionuclide COCs were } \\
\text { identified for the } 100-\mathrm{K}-55: 1 \text { and } \\
\text { 100-K-56:1 sites. Total excess } \\
\text { cancer risk values for the } 116-\mathrm{KW}-4 \\
\text { and } 116-\mathrm{KE}-5 \text { sites are } 1.1 \times 10^{-7} \\
\text { and } 1.6 \times 10^{-7} \text {, respectively. }\end{array}$ & & c \\
\hline \multirow{2}{*}{$\begin{array}{l}\text { Groundwater/River } \\
\text { Protection - } \\
\text { Radionuclides }\end{array}$} & $\begin{array}{l}\text { 1. Attain single-COC groundwater } \\
\text { and river protection RAGs. }\end{array}$ & $\begin{array}{l}\text { 1. All single-COC groundwater and } \\
\text { river RAGs have been attained. }\end{array}$ & \multirow[t]{2}{*}{ Yes } & $d, e$ \\
\hline & $\begin{array}{l}\text { 2. Attain National Primary Drinking } \\
\text { Water Standards: } 4 \mathrm{mrem} / \mathrm{yr} \\
\text { (beta/gamma) dose rate to target } \\
\text { receptor/organs. }\end{array}$ & $\begin{array}{l}\text { 2. All organ-specific dose rates are } \\
\text { below the } 4 \mathrm{mrem} / \mathrm{yr} \text { dose rate limit. }\end{array}$ & & $d, e$ \\
\hline
\end{tabular}




\section{Table ES-1. Summary of Cleanup Verification Results for the 100-K-55:1, 100-K-56:1, 116-KW-4, and 116-KE-5 Sites. (2 Pages)}

\begin{tabular}{|c|c|c|c|c|}
\hline $\begin{array}{l}\text { Regulatory } \\
\text { Requirement }\end{array}$ & Remedial Action Goals & Results & $\begin{array}{l}\text { Remedial } \\
\text { Action } \\
\text { Objectives } \\
\text { Attained? }\end{array}$ & Ref. \\
\hline & $\begin{array}{l}\text { 3. Meet drinking water standards for } \\
\text { alpha emitters: the more stringent } \\
\text { of the } 15 \mathrm{pCi} / \mathrm{L} \mathrm{MCL} \text { or } 1 / 25 \text { th of } \\
\text { the derived concentration guide } \\
\text { per DOE Order } 5400.5 \text {. }\end{array}$ & $\begin{array}{l}\text { 3. No alpha-emitting COCs were } \\
\text { identified for the } 100-\mathrm{K}-55: 1 \text {, } \\
100-\mathrm{K}-56: 1,116-\mathrm{KW}-4 \text {, or } 116-\mathrm{KE}-5 \\
\text { sites. }\end{array}$ & & \\
\hline & $\begin{array}{l}\text { 4. Meet total uranium standard of } \\
21.2 \mathrm{pCi} / \mathrm{L} \text {. }\end{array}$ & $\begin{array}{l}\text { 4. Uranium was not identified as a } \\
\text { COC for the } 100-\mathrm{K}-55: 1,100-\mathrm{K}-56: 1 \text {, } \\
116-\mathrm{KW}-4 \text {, or } 116-\mathrm{KE}-5 \text { sites. }\end{array}$ & & \\
\hline $\begin{array}{l}\text { Groundwater/River } \\
\text { Protection - } \\
\text { Nonradionuclides }\end{array}$ & $\begin{array}{l}\text { 1. Attain individual nonradionuclide } \\
\text { groundwater and river cleanup } \\
\text { requirements. }\end{array}$ & $\begin{array}{l}\text { 1. All the groundwater and river RAGs } \\
\text { have been attained. }\end{array}$ & Yes & \\
\hline \multirow{6}{*}{$\begin{array}{l}\text { Other supporting } \\
\text { Information }\end{array}$} & \multicolumn{3}{|c|}{ 1. 100-K-55:1 and overburden $95 \%$ UCL calculation (Appendix C). } & $g$ \\
\hline & \multicolumn{3}{|c|}{ 2. 100-K-56:1 95\% UCL calculation (Appendix C). } & h \\
\hline & \multicolumn{3}{|c|}{ 3. 100-K-55:1 and overburden sample variance calculation (Appendix C). } & $\mathrm{i}$ \\
\hline & \multicolumn{3}{|c|}{ 4. 100-K-56:1 sample variance calculation (Appendix C). } & j \\
\hline & \multicolumn{3}{|c|}{ 5. 100-K-55:1 and overburden verification sample location design (Appendix C). } & $\mathrm{k}$ \\
\hline & \multicolumn{3}{|c|}{ 6. 100-K-56:1 verification sample location design (Appendix C). } & $L$ \\
\hline
\end{tabular}

"100-K-55:1 Pipeline RESRAD Calculation, 0100K-CA-V0046, Rev. 0, Bechtel Hanford, Inc., Richland, Washington.

b 100-K-56:1 Pipeline RESRAD Calculation, 0100K-CA-V0050, Rev. 0, Bechtel Hanford, Inc., Richland, Washington.

c 116-KW-4 and 116-KE-5 Heat Recovery Stations Hazard Quotient and Carcinogenic Risk Calculations, 0100K-CA-V0054, Rev. 0, Bechtel Hanford, Inc., Richland, Washington.

¿ 100-K-55:1 Comparison to Drinking Water Standards (MCL) Calculation Brief, 0100K-CA-V0047, Rev. 0, Bechtel Hanford, Inc., Richland, Washington.

e 100-K-56:1 Comparison to Drinking Water Standards (MCL) Calculation, 0100K-CA-V0051, Rev. 0, Bechtel Hanford, Inc., Richland, Washington.

'Uranium limits selected in the Amendment to the Interim Action Record of Decision for the 100-BC-1, 100-DR-1, and 100-HR-1 Operable Units, Hanford Site, Benton County, Washington (ROD) (EPA 1997) and Remedial Design Report/Remedial Action Work Plan for the 100 Area (DOE-RL 2005b) were based on 1/25th of the derived concentration guidelines from DOE Order 5400.5 . Since the time of ROD signature, the U.S. Environmental Protection Agency has promulgated a more restrictive $\mathrm{MCL}$ of $30 \mu \mathrm{g} / \mathrm{L}$ for total uranium (65 Federal Register 76708). Based on the isotopic distribution of uranium in the 100 Areas, the $30 \mu \mathrm{g} / \mathrm{L} \mathrm{MCL}$ corresponds to $21.2 \mathrm{pCi} / \mathrm{L}$. Concentration-to-activity calculations are documented in Calculation of Total Uranium Activity Corresponding to a Maximum Contaminant Level for Total Uranium of 30 Micrograms per Liter in Groundwater, 0100X-CA-V0038 (BHI 2001).

'100-K-55:1 Pipeline Cleanup Verification 95\% UCL Calculation, 0100K-CA-V0045, Rev. 0, Bechtel Hanford, Inc., Richland, Washington.

h 100-K-56:1 Pipeline Cleanup Verification 95\% UCL Calculation, 0100K-CA-V0049, Rev. 0, Bechtel Hanford, Inc., Richland, Washington.

' 100-K-55 Pipeline and Overburden Variance Calculation, 0100K-CA-V0041, Rev. 0, Bechtel Hanford, Inc., Richland, Washington.

100-K-56:1 Pipeline Variance Calculation, 0100K-CA-V0052, Rev. 0, Bechtel Hanford, Inc., Richland, Washington.

k 100-KW-55 Pipelines Shallow, Deep, and Overburden Zone Sampling Plan, 0100K-CA-V0039, Rev. 0, Bechtel Hanford, Inc., Richland, Washington.

L 100-K-56:1 Pipelines Shallow and Deep Zone Sampling Plan, 0100K-CA-V0053, Rev. 0, Bechtel Hanford, Inc., Richland, Washington. $\mathrm{COC}=$ contaminant of concern

$\mathrm{COPC}=$ contaminant of potential concern

RAG = remedial action goal

MCL = maximum contaminant level (drinking water standard)

RESRAD = RESidual RADioactivity (dose model)

$\mathrm{UCL}=$ upper confidence limit 
CVP-2005-00006

Rev. 0

ES-4 
CVP-2005-00006

Rev. 0

\section{Attachment ES-1 \\ Waste Site Reclassification Form}

\begin{tabular}{|c|c|c|}
\hline $\begin{array}{l}\text { Date Submitted: } \\
09 / 21 / 05 \\
\text { Originator: } \\
\text { R. A. Carlson } \\
\text { Phone: } 373-1440\end{array}$ & $\begin{array}{l}\text { Operable Unit(s): 100-KR-2 } \\
\text { Waste Site ID: 100-K-55:1 } \\
\text { Type of Reclassification Action: } \\
\text { Rejected } \\
\text { Closed Out } \\
\text { Interim Closed Out } \\
\text { No Action }\end{array}$ & $\begin{array}{l}\text { Control Number: } 2005-029 \\
\text { Lead Agency: EPA }\end{array}$ \\
\hline \multicolumn{3}{|c|}{$\begin{array}{l}\text { This form documents agreement among the parties listed below authorizing classification of the subject unit as } \\
\text { rejected, closed out, or no action and authorizing backfill of the site, if appropriate. Final removal from the National } \\
\text { Priorities List of no action or closed-out sites will occur at a future date. }\end{array}$} \\
\hline \multicolumn{3}{|c|}{$\begin{array}{l}\text { Remedial actions at this site ha } \\
\text { established by the U.S. Environ } \\
\text { Office, in concurrence with the } \\
\text { (1) excavating the site to the ex } \\
\text { excavation materials at the Env } \\
\text { (3) backfilling the site with clear } \\
\text { completed. } \\
\text { Basis for reclassification: }\end{array}$} \\
\hline \multicolumn{3}{|c|}{$\begin{array}{l}\text { The 100-K-55:1 pipelines have been remediated to meet the cleanup standards specified in the } 1997 \text { Amendment to } \\
\text { the Interim Action Record of Decision for the 100-BC-1, 100-DR-1, and 100-HR-1 Operable Units, Hanford Site, } \\
\text { Benton County, Washington, U.S. Environmental Protection Agency, Region } 10 \text {, Seattle, Washington } \\
\text { (EPA/AMD/R10-97/044). Remedial actions were performed so as to not preclude any future uses (as bounded by } \\
\text { the rural-residential scenario), to allow unrestricted use of shallow zone soils (i.e., surface to } 4.6 \mathrm{~m}[15 \mathrm{ft} \text { deep), and } \\
\text { to protect groundwater and the Columbia River. Institutional controls are required for the site to prevent drilling or } \\
\text { excavation into deep zone soils. The basis for reclassification is described in detail in the Cleanup Verification } \\
\text { Package for the 100-K-55:1 and 100-K-56:1 Pipelines and the 116-KW-4 and 116-KE-5 Heat Recovery Stations } \\
\text { (CVP-2005-00006), Washington Closure Hanford, Richland, Washington. }\end{array}$} \\
\hline J. Zeisloft & & 9|22|05 \\
\hline $\begin{array}{l}\text { DOE-RL Project Manager } \\
\text { NA }\end{array}$ & Sighature & Dale \\
\hline $\begin{array}{l}\text { Ecology Project Manager } \\
\text { L. E. Gadbois }\end{array}$ & $\begin{array}{l}\text { Signature } \\
\text { Xauree }\end{array}$ & $9-23-2005$ \\
\hline EPA Project Manager & Signature & Date \\
\hline
\end{tabular}


CVP-2005-00006

Rev. 0

ES-6 
CVP-2005-00006

Rev. 0

\section{Attachment ES-2}

Waste Site Reclassification Form

\begin{tabular}{|l|l|l|}
\hline Date Submitted: & $\begin{array}{l}\text { Operable Unit(s): 100-KR-2 } \\
\text { 09/21/05 }\end{array}$ & $\begin{array}{l}\text { Control Number: 2005-030 } \\
\text { Lead Agency: EPA }\end{array}$ \\
Originator: & $\begin{array}{l}\text { Waste Site ID: 100-K-56:1 } \\
\text { R. A. Carlson }\end{array}$ & \\
Phone: $373-1440$ & $\begin{array}{ll}\text { Type of Reclassification Action: } \\
\text { Rejected } \\
\text { Closed Out } \\
\text { Interim Closed Out } \\
\text { No Action }\end{array}$ & $\square$ \\
& & $\square$
\end{tabular}

This form documents agreement among the parties listed below authorizing classification of the subject unit as rejected, closed out, or no action and authorizing backfill of the site, if appropriate. Final removal from the National Priorities List of no action or closed-out sites will occur at a future date.

\section{Description of current waste site condition:}

Remedial actions at this site have been performed in accordance with remedial action objectives and goals established by the U.S. Environmental Protection Agency and the U.S. Department of Energy, Richland Operations Office, in concurrence with the Washington State Department of Ecology. The selected remedial action involves (1) excavating the site to the extent required to meet specified soil cleanup levels, (2) disposing of contaminated excavation materials at the Environmental Restoration Disposal Facility in the 200 Area of the Hanford Site, and (3) backfilling the site with clean soil to adjacent grade elevations. The excavation and disposal activities have been completed.

\section{Basis for reclassification:}

The 100-K-56:1 pipelines have been remediated to meet the cleanup standards specified in the 1997 Amendment to the Interim Action Record of Decision for the 100-BC-1, 100-DR-1, and 100-HR-1 Operable Units, Hanford Site, Benton County, Washington, U.S. Environmental Protection Agency, Region 10, Seattle, Washington (EPAAMD/R10-97/044). Remedial actions were performed so as to not preclude any future uses (as bounded by the rural-residential scenario), to allow unrestricted use of shallow zone soils (i.e., surface to $4.6 \mathrm{~m}$ [15 ft] deep), and to protect groundwater and the Columbia River. Institutional controls are required for the site to prevent drilling or excavation into deep zone soils. The basis for reclassification is described in detail in the Cleanup Verification Package for the 100-K-55:1 and 100-K-56:1 Pipelines and the 116-KW-4 and 116-KE-5 Heat Recovery Stations (CVP-2005-00006), Washington Closure Hanford, Richland, Washington.

J. Zeisloft

DOE-RL Project Manager

NA

Ecology Project Manager

L. E. Gadbois

EPA Project Manager

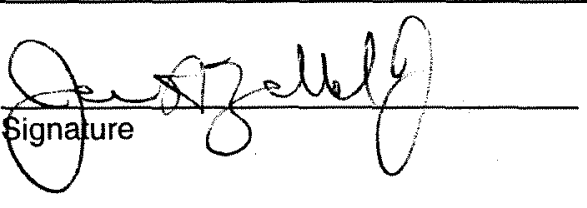

Signature

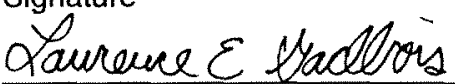

Signature

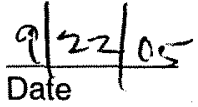

Date

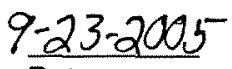

Date 
CVP-2005-00006

Rev. 0

ES-8 
CVP-2005-00006

Rev. 0

\section{Attachment ES-3 \\ Waste Site Reclassification Form}

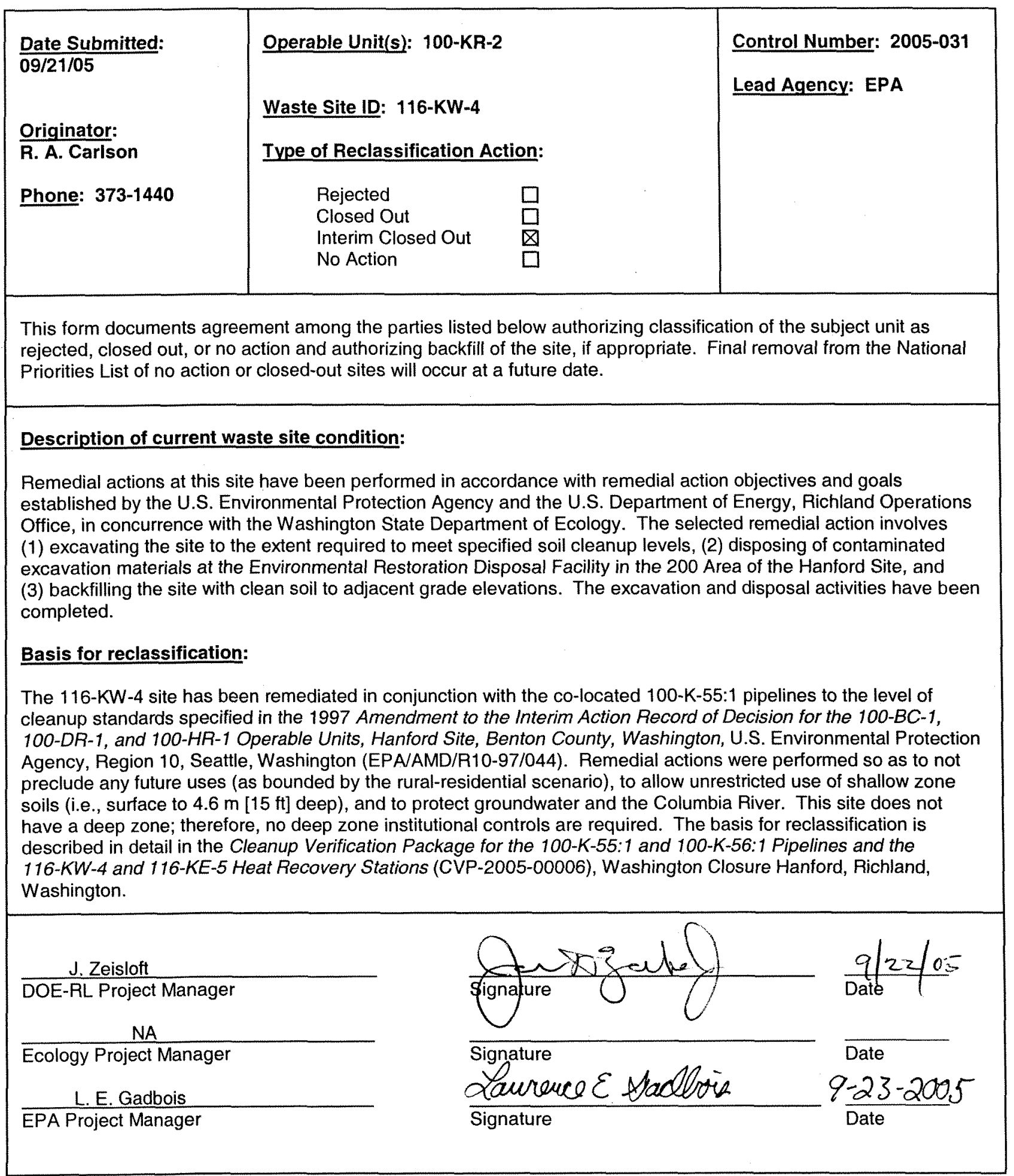


CVP-2005-00006

Rev. 0

ES-10 
CVP-2005-00006

Rev. 0

\section{Attachment ES-4 \\ Waste Site Reclassification Form}

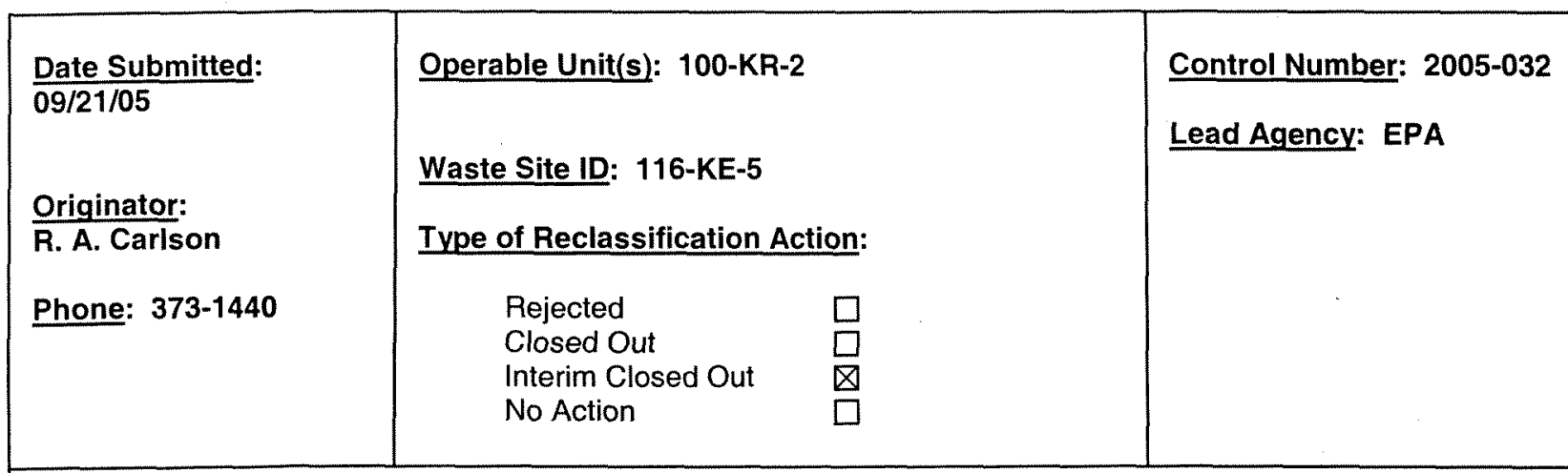

This form documents agreement among the parties listed below authorizing classification of the subject unit as rejected, closed out, or no action and authorizing backfill of the site, if appropriate. Final removal from the National Priorities List of no action or closed-out sites will occur at a future date.

\section{Description of current waste site condition:}

Remedial actions at this site have been performed in accordance with remedial action objectives and goals established by the U.S. Environmental Protection Agency and the U.S. Department of Energy, Richland Operations Office, in concurrence with the Washington State Department of Ecology. The selected remedial action involves (1) excavating the site to the extent required to meet specified soil cleanup levels, (2) disposing of contaminated excavation materials at the Environmental Restoration Disposal Facility in the 200 Area of the Hanford Site, and (3) backfilling the site with clean soil to adjacent grade elevations. The excavation and disposal activities have been completed.

\section{Basis for reclassification:}

The 116-KE-5 site has been remediated in conjunction with the co-located 100-K-56:1 pipelines to the level of cleanup standards specified in the 1997 Amendment to the Interim Action Record of Decision for the 100-BC-1, 100-DR-1, and 100-HR-1 Operable Units, Hanford Site, Benton County, Washington, U.S. Environmental Protection Agency, Region 10, Seattle, Washington (EPA/MD/R10-97/044). Remedial actions were performed so as to not preclude any future uses (as bounded by the rural-residential scenario), to allow unrestricted use of shallow zone soils (i.e., surface to $4.6 \mathrm{~m}$ [15 ft] deep), and to protect groundwater and the Columbia River. This site does not have a deep zone; therefore, no deep zone institutional controls are required. The basis for reclassification is described in detail in the Cleanup Verification Package for the 100-K-55:1 and 100-K-56:1 Pipelines and the 116-KW-4 and 116-KE-5 Heat Recovery Stations (CVP-2005-00006), Washington Closure Hanford, Richland, Washington.

\section{J. Zeisloft}

DOE-RL Project Manager

NA

Ecology Project Manager

L. E. Gadbois

EPA Project Manager

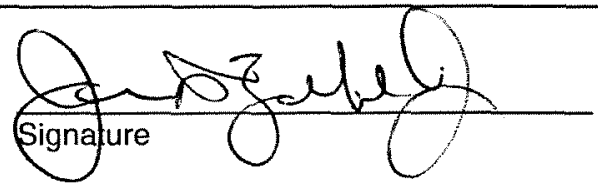

Signature

otareuce 5 thatoris

Signature

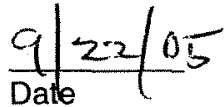

Date

$9-23-2005$

Date 
CVP-2005-00006

Rev. 0

ES-12 


\section{CONTENTS}

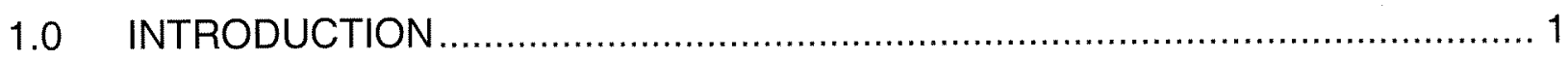

2.0 SITE DESCRIPTION AND SUPPORTING INFORMATION ............................ 2

3.0 REMEDIAL ACTION FIELD ACTIVITIES .................................................. 5

3.1 EXCAVATION AND DISPOSAL .................................................... 5

3.2 FIELD SCREENING AND VARIANCE SAMPLING ............................ 12

3.3 CLEANUP VERIFICATION SAMPLING AND ANALYSIS .................... 12

4.0 CLEANUP VERIFICATION DATA EVALUATION ….................................... 13

4.1 DATA QUALITY ASSESSMENT PROCESS ................................... 13

4.2 CONTAMINANTS OF CONCERN 95\% UPPER CONFIDENCE LIMIT ... 14

4.3 SITE-SPECIFIC CLEANUP VERIFICATION MODEL …..................... 17

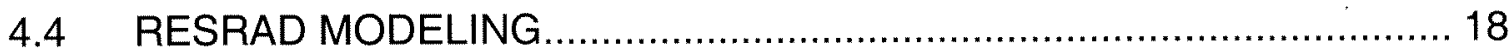

5.0 EVALUATION OF REMEDIAL ACTION GOAL ATTAINMENT ....................... 18

5.1 DIRECT EXPOSURE SOIL REMEDIAL ACTION GOALS ATTAINED.... 18

5.1 .1 Radionuclides .................................................................. 18

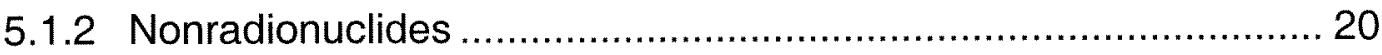

5.2 GROUNDWATER REMEDIAL ACTION GOALS ATTAINED ….............. 22

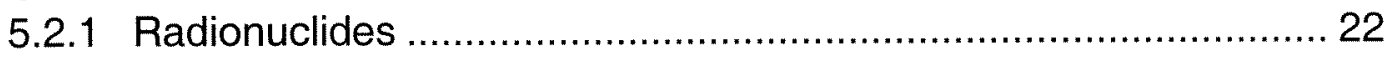

5.2.2 Nonradionuclides ................................................................. 23

5.3 COLUMBIA RIVER REMEDIAL ACTION GOALS ATTAINED ................ 24

5.3.1 Radionuclides ................................................................. 24

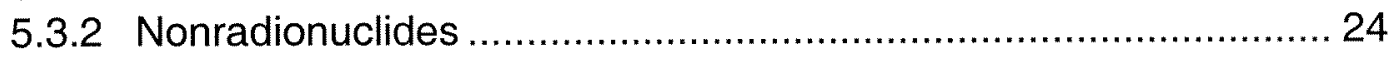

5.4 WAC 173-340 THREE-PART TEST FOR NONRADIONUCLIDES ......... 25

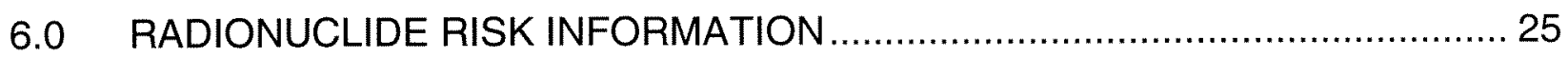

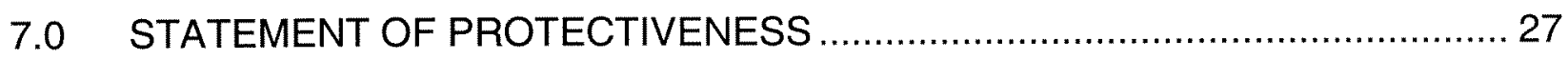

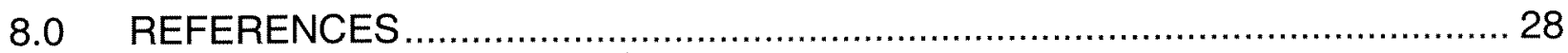




\section{APPENDICES}

A SUMMARY OF VERIFICATION SOIL SAMPLING AND

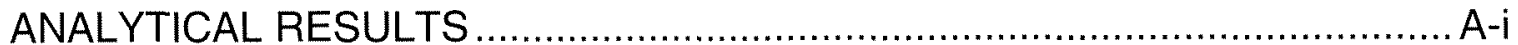

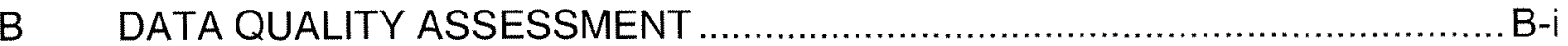

C RESRAD INPUT PARAMETERS AND CALCULATION BRIEF EXCERPTS ......

C1 100-K-55 Pipeline and Overburden Variance Calculation (0100K-CA-V0041) ....................................................................

C2 100-KW-55 Pipelines Shallow, Deep, and Overburden Zone Sampling Plan (0100K-CA-V0039) ................................................6-67

C3 100-K-55:1 Pipeline Cleanup Verification 95\% UCL Calculation (0100K-CA-V0045) C-81

C4 100-K-55:1 Pipeline RESRAD Calculation (0100K-CA-V0046) ..............-91

C5 100-K-55:1 Comparison to Drinking Water Standards (MCL) Calculation Brief (0100K-CA-V0047) ............................................... -99

C6 100-K-56:1 Pipeline Variance Calculation (0100K-CA-V0052) ............-103

C7 100-K-56:1 Pipelines Shallow and Deep Zone Sampling Plan (0100K-CA-V0053)

C8 100-K-56:1 Pipeline Cleanup Verification 95\% UCL Calculation (0100K-CA-V0049)

C9 100-K-56:1 Pipeline RESRAD Calculation (0100K-CA-V0050) ...........C-127

C10 100-K-56:1 Comparison to Drinking Water Standards (MCL) Calculation (0100K-CA-V0051) C-133

C11 116-KW-4 and 116-KE-5 Heat Recovery Stations Hazard Quotient and Carcinogenic Risk Calculation (0100K-CA-V0054) 


\section{FIGURES}

1. Hanford Site Map and 100-K-55 and 116-KW-4 Site Plans................................ 3

2. Hanford Site Map and 100-K-56 and 116-KE-5 Site Plans................................ 4

3. Pre-Remediation Topographic Plan for the 100-K-55 Pipelines and

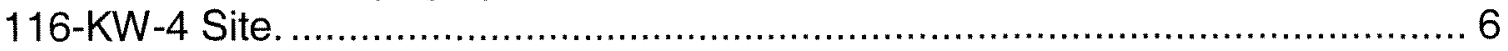

4. Pre-Remediation Topographic Plan for the Western 100-K-56 Pipelines and 116-KE-5 Site........................................................................................ 7

5. Pre-Remediation Topographic Plan for the Eastern 100-K-56 Pipelines............ 8

6. Post-Remediation Topographic Plan for the 100-K-55 Pipelines and

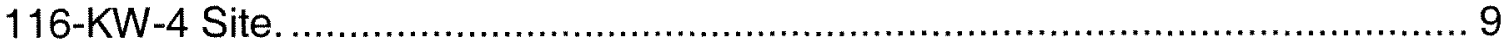

7. Post-Remediation Topographic Plan for the Western 100-K-56 Pipelines and 116-KE-5 Site ................................................................................... 10

8. Post-Remediation Topographic Plan for the Eastern 100-K-56:1 Pipelines. ..... 11

9. Combined Shallow and Deep Zone Dose Rate Estimates for the 100-K-55:1 Site (All Radionuclides, All Pathways). ............................................ 19

10. Combined Shallow and Deep Zone Dose Rate Estimates for the 100-K-56:1 Site (All Radionuclides, All Pathways).

11. Overburden Dose Rate Estimates for the 100-K-55:1 and 100-K-56:1 Sites (All Radionuclides, All Pathways).............................................................. 20

12. Dose Rates to Organs from Groundwater Impacted by the 100-K-55:1 Site...... 23

13. Dose Rates to Organs from Groundwater Impacted by the 100-K-56:1 Site...... 23

14. Combined Radionuclide Excess Lifetime Cancer Risk for the 100-K-55:1 Shallow and Deep Zone Decision Units. ................................................... 26

15. Combined Radionuclide Excess Lifetime Cancer Risk for the 100-K-56:1 Shallow and Deep Zone Decision Units. 26

16. Radionuclide Excess Lifetime Cancer Risk for the 100-K-55:1 and 100-K-56:1 Sites Overburden Material.

\section{TABLES}

1. 100-K-55:1 and 100-K-56:1 Remedial Action Goals....................................... 1

2. 116-KW-4 and 116-KE-5 Remedial Action Goals.......................................... 2

3. 100-K-55:1 and 100-K-56:1 Cleanup Verification Data Set............................ 14

4. Maximum Concentrations at the Former 116-KW-3 and 116-KE-4 Retention Basin Discharge Points. ............................................................................ 16

5. 116-KW-4 and 116-KE-5 Cleanup Verification Data Set. ............................... 17

6. Estimated Peak Radionuclide Groundwater Concentrations Compared to Remedial Action Goals. 
CVP-2005-00006

Rev. 0 


\section{ACRONYMS AND ABBREVIATIONS}

$\begin{array}{ll}\text { COC } & \text { contaminant of concern } \\ \text { COPC } & \text { contaminant of potential concern } \\ \text { CVP } & \text { cleanup verification package } \\ \text { DQA } & \text { data quality assessment } \\ \text { EPA } & \text { U.S. Environmental Protection Agency } \\ \text { ERDF } & \text { Environmental Restoration Disposal Facility } \\ \text { Nal } & \text { sodium iodide } \\ \text { RAG } & \text { remedial action goal } \\ \text { RAO } & \text { remedial action objective } \\ \text { RDR/RAWP } & \text { remedial design report/remedial action work plan } \\ \text { RESRAD } & \text { RESidual RADioactivity (dose assessment model) } \\ \text { ROD } & \text { record of decision } \\ \text { SAP } & \text { sampling and analysis plan } \\ \text { UCL } & \text { upper confidence limit } \\ \text { WAC } & \text { Washington Administrative Code }\end{array}$


CVP-2005-00006

Rev. 0 


\subsection{INTRODUCTION}

This cleanup verification package (CVP) documents that the 100-K-55:1 and 100-K-56:1 pipelines were remediated in accordance with the Amendment to the Interim Action Record of Decision for the 100-BC-1, 100-DR-1, and 100-HR-1 Operable Units, Hanford Site, Benton County, Washington (ROD) (EPA 1997). Remedial action objectives (RAOs) and remedial action goals (RAGs) for these sites are documented in the ROD (EPA 1997) and the Remedial Design Report/Remedial Action Work Plan for the 100 Area (RDR/RAWP) (DOE-RL 2005b). The ROD provides the U.S. Department of Energy, Richland Operations Office the authority, guidance, and objectives to conduct this remedial action. This CVP also documents that the 116-KW-4 and 116-KE-5 sites were remediated as part of remedial efforts for the 100-K-55:1 and 100-K-56:1 pipelines.

The remedy specified in the ROD and conducted for the 100-K-55:1 and 100-K-56:1 pipelines and co-located 116-KW-4 and 116-KE-5 sites included (1) excavating the sites to the extent required to meet specified soil cleanup levels, (2) disposing of contaminated excavation materials at the Environmental Restoration Disposal Facility (ERDF) in the 200 Area of the Hanford Site, and (3) backfilling the site with clean soil to average adjacent grade elevation. Excavation was driven by RAOs for direct exposure, protection of groundwater, and protection of the Columbia River. For the respective points of compliance, RAGs summarized in Table 1 were established for the contaminants of concern (COCs) in the RDR/RAWP (DOE-RL 2005b). Waste site COCs listed in Table 1 were identified in the 100 Area Remedial Action Sampling and Analysis Plan (SAP) (DOE-RL 2001a) for the 100-K-55 and 100-K-56 pipelines. No documented COCs exist for the 116-KW-4 and 116-KE-5 sites but, based on operational knowledge, ethylene glycol was identified as the primary potential residual contaminant for these sites. In addition, residual concentrations of arsenic, barium, cadmium, hexavalent chromium, total chromium, mercury, and lead were also evaluated for these sites. The RAGs for these contaminants of potential concern (COPCs) are summarized in Table 2.

Table 1. 100-K-55:1 and 100-K-56:1 Remedial Action Goals.

\begin{tabular}{|c|c|c|c|}
\hline cocs & $\begin{array}{l}\text { Direct Exposure } \\
\mathrm{RAG}^{\mathrm{a}}\end{array}$ & 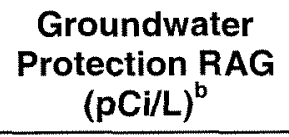 & $\begin{array}{l}\text { Columbia River } \\
\text { Protection RAG } \\
\qquad(\mathrm{pCi} / \mathrm{L})^{\mathrm{b}}\end{array}$ \\
\hline Cesium-137 & \multirow{3}{*}{$\begin{array}{l}15 \mathrm{mrem} / \mathrm{yr} \\
\text { (cumulative) }^{\mathrm{a}}\end{array}$} & \multirow{3}{*}{$\begin{array}{c}4 \mathrm{mrem} / \mathrm{yr} \\
\text { (cumulative) }^{\mathrm{b}}\end{array}$} & \multirow{3}{*}{$\begin{array}{c}4 \mathrm{mrem} / \mathrm{yr} \\
\text { (cumulative) }^{\mathrm{b}}\end{array}$} \\
\hline Europium-152 & & & \\
\hline Europium-154 & & & \\
\hline
\end{tabular}

${ }^{a}$ Lookup values that correspond to the $15 \mathrm{mrem} / \mathrm{yr}$ dose rate are based on a generic site model and are presented in the Remedial Design Report/Remedial Action Work Plan for the 100 Area (RDR/RAWP) (DOE-RL 2005b).

${ }^{b}$ Lookup values that correspond to the individual radionuclide $4 \mathrm{mrem} / \mathrm{yr}$ dose rate equivalent for beta- and gamma-emitter RAGs per National Drinking Water Standards as presented in the RDR/RAWP (DOE-RL 2005b) and Table 5 of this cleanup verification package.

$\mathrm{COC}=$ contaminant of concern

$R A G=$ remedial action goal 
Table 2. 116-KW-4 and 116-KE-5 Remedial Action Goals.

\begin{tabular}{|l|c|c|c|}
\hline \multicolumn{1}{|c|}{ COPCs } & $\begin{array}{c}\text { Direct Exposure } \\
\text { RAG } \\
(\mathbf{m g} / \mathbf{k g})\end{array}$ & $\begin{array}{c}\text { Soil RAG for } \\
\text { Groundwater Protection } \\
(\mathbf{m g} / \mathbf{k g})\end{array}$ & $\begin{array}{c}\text { Soil RAG for Columbia } \\
\text { River Protection } \\
\text { (mg/kg) }\end{array}$ \\
\hline Arsenic & $20^{\mathrm{a}}$ & $20^{\mathrm{a}}$ & $20^{\mathrm{a}}$ \\
\hline Barium & $5,600^{\mathrm{b}}$ & $132^{\mathrm{c}}$ & 224 \\
\hline Cadmium & $13.9^{\mathrm{d}}$ & $0.81^{\mathrm{c}}$ & $0.81^{\mathrm{c}}$ \\
\hline Ethylene glycol & $160,000^{\mathrm{b}}$ & 3,200 & 6,400 \\
\hline Hexavalent chromium & $2.1^{\mathrm{e}}$ & $8^{\mathrm{g}}$ & $2^{\mathrm{h}}$ \\
\hline Total chromium & $240^{\mathrm{f}}$ & $18.5^{\mathrm{c}}$ & $18.5^{\mathrm{c}}$ \\
\hline Lead & $80,000^{\mathrm{b}}$ & $10.2^{\mathrm{c}}$ & $10.2^{\mathrm{c}}$ \\
\hline Mercury & $353^{\mathrm{h}}$ & $0.33^{\mathrm{c}}$ & $0.33^{\mathrm{c}}$ \\
\hline
\end{tabular}

${ }^{\mathrm{a}}$ The cleanup level of $20 \mathrm{mg} / \mathrm{kg}$ has been agreed to by the Tri-Party project managers (DOE-RL 2005b).

${ }^{b}$ Noncarcinogenic cleanup level calculated from WAC 173-340-740(3), Method B, 1996.

${ }^{c}$ Where cleanup levels are less than background, cleanup levels default to background (WAC 173-340-700[4][d]) (1996).

'Value calculated based on the inhalation exposure pathway per WAC 173-340-750(4)(b)(ii)(A) or (B).

${ }^{\circ}$ Calculation of Hexavalent Chromium Carcinogenic Risk, 0100X-CA-V0031 (BHI 2000).

'WAC 173-340-750(3) Method B noncarcinogenic cleanup limit.

9 Soil RAG based on "100 times groundwater cleanup" rule as presented in the Remedial Design Report/Remedial Action Work Plan for the 100 Area (RDR/RAWP) (DOE-RL 2005b).

${ }^{n}$ Soil RAG based on 100 times dilution attenuation factor times surface water quality standard as presented in the RDR/RAWP (DOE-RL 2005b).

'A WAC 173-340-740(3) (1996) value for lead is not available. This value is based on the Guidance Manual for the Integrated Exposure Uptake Biokinetic Model for Lead in Children (EPA 1994).

$\mathrm{COPC}=$ contaminant of potential concern

$\mathrm{RAG}=$ remedial action goal

WAC $=$ Washington Administrative Code

\subsection{SITE DESCRIPTION AND SUPPORTING INFORMATION}

The 100-K-55 and 100-K-56 pipelines and the 116-KW-4 and 116-KE-5 sites are all part of the 100-KR-2 Operable Unit in the 100-K Area. The 100-K-55 pipelines consist of the gravity-flow process effluent pipelines that formerly serviced the 105-KW Reactor (Figure 1), terminating at the 116-K-1 Crib, the 116-K-2 Trench, and the $116-\mathrm{KW}-3$ retention basins. The $100-\mathrm{K}-56$ pipelines were the equivalent process effluent pipelines for the 105-KE Reactor (Figure 2), terminating at the $116-\mathrm{K}-1 \mathrm{Crib}$, the 116-K-2 Trench, and the 116-KE-4 retention basins. These pipelines consisted primarily of carbon steel piping, ranging in size from $0.08 \mathrm{~m}$ (3 in.) to $1.83 \mathrm{~m} \mathrm{(72} \mathrm{in.).}$ A $0.61-m(24-i n$.) process water pipeline connecting the two reactor buildings is also administratively part of the $100-\mathrm{K}-55$ and $100-\mathrm{K}-56$ sites. 
Figure 1. Hanford Site Map and 100-K-55 and 116-KW-4 Site Plans.

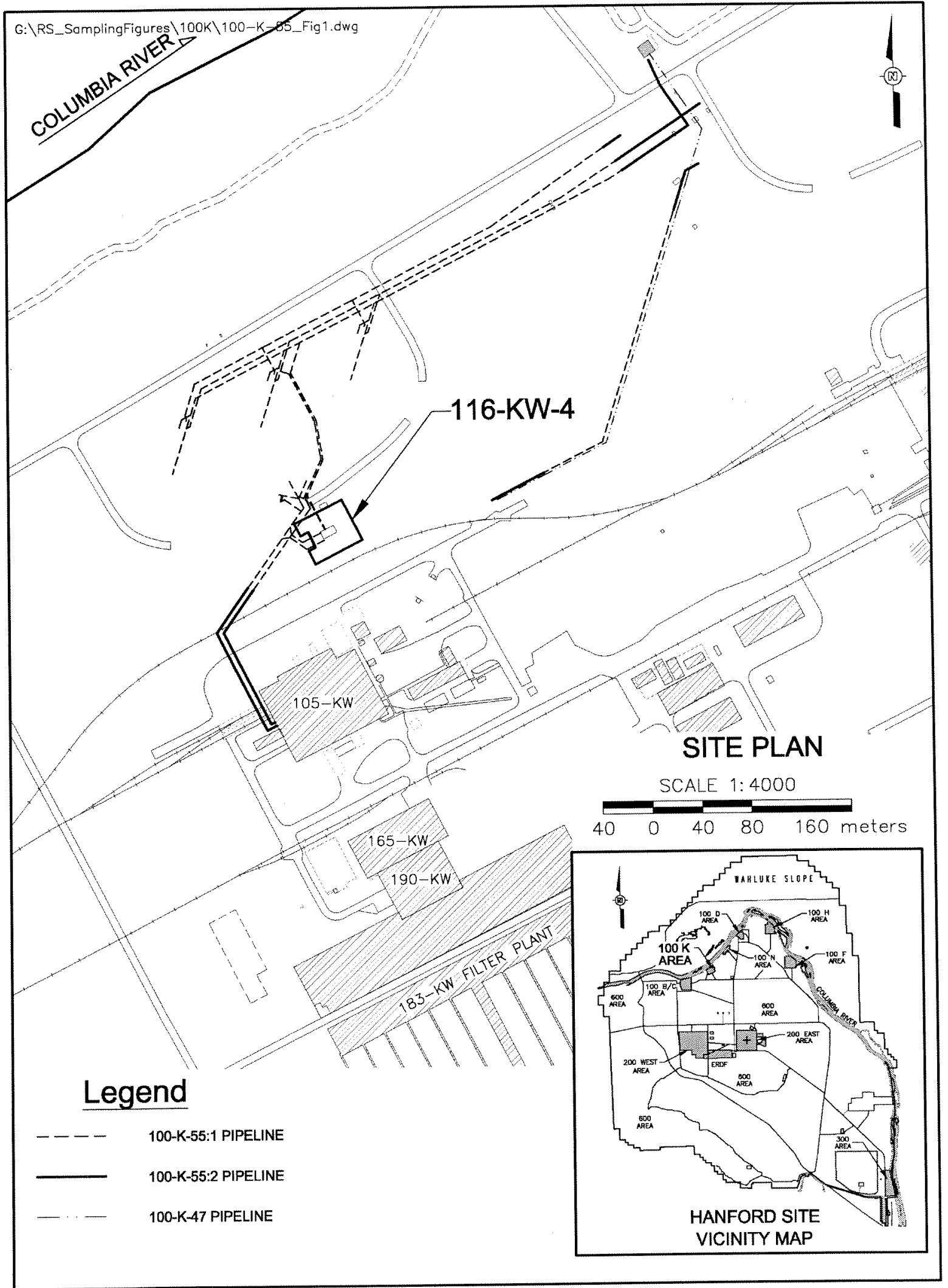


Figure 2. Hanford Site Map and 100-K-56 and 116-KE-5 Site Plans.

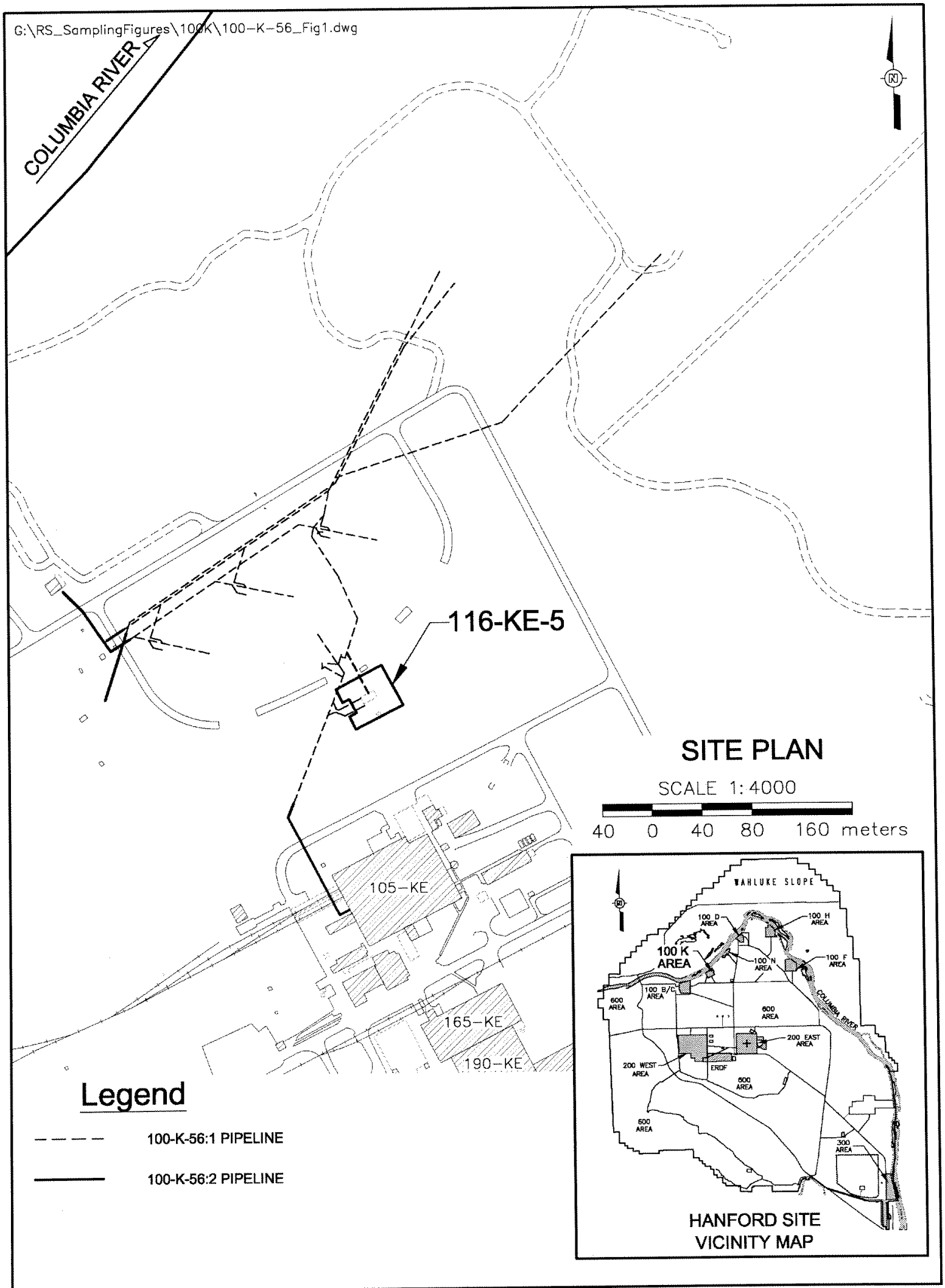


The 100-K-55 and 100-K-56 pipelines have been divided into subsites to address the current extent of remedial action. Remediation of the full lengths of the pipelines has not been completed in the interests of preserving reactor security fences and active subsurface utility features. The portions of the pipelines addressed within this CVP have been identified as the 100-K-55:1 and 100-K-56:1 pipelines, with administrative boundaries indicated in Figures 1 and 2 . Closeout of the remaining 100-K-55:2 and 100-K-56:2 pipelines will be addressed separately.

The 116-KW-4 and 116-KE-5 sites consist of the former heat recovery stations associated with the $100-\mathrm{K}-55$ and $100-\mathrm{K}-56$ pipelines, respectively. The facilities at these sites consisted of heat exchangers using an ethylene glycol solution to recover heat for space heating and process requirements in 100-K Area facilities. The heat exchangers were removed from these facilities prior to remedial action, leaving residual piping at the sites.

\subsection{REMEDIAL ACTION FIELD ACTIVITIES}

\subsection{EXCAVATION AND DISPOSAL}

Remedial action activities at the $100-\mathrm{K}$ reactor effluent pipelines began on December 9 , 2002. Remediation of the pipelines involved excavation and staging of overburden material and removal of contaminated piping, debris, and soil. Contaminated materials were disposed at the ERDF.

Remedial action excavation was completed on April 15, 2005. Pre- and post-remediation topographic maps are shown in Figures 3 through 8 . Note that the Waste Information Data System boundaries for the 116-KW-4 and 116-KE-5 sites are much larger than the actual footprint of the heat recovery facilities. The soil area beneath each of the former heat recovery stations was excavated in its entirety with the removal of the co-located pipelines. Approximately $55,960 \mathrm{~m}^{2}\left(602,350 \mathrm{ft}^{2}\right)$ of plan area was excavated, including excavation within the deep zone (greater than $4.6 \mathrm{~m}[15 \mathrm{ft}]$ below ground surface) up to $8.8 \mathrm{~m}(29 \mathrm{ft})$ below ground surface where necessary to complete remediation. Approximately 86,551 metric tons $(95,406$ U.S. tons) of material from the sites was removed and disposed at the ERDF.

The active 100-K-47 concrete culvert shown in Figures 3 and 6 was not removed during remediation of the adjacent section of the 100-K-55:1 pipeline. Verification samples collected adjacent to the culvert (shallow zone sample areas $E 10$ and $F 1$ through $F 4$ and deep zone sample areas B4 through B6, C7 through C9, D1, D2, and D10) did not indicate contamination associated with the culvert (see Appendix A). 
CVP-2005-00006

Rev. 0

Figure 3. Pre-Remediation Topographic Plan for the 100-K-55 Pipelines and 116-KW-4 Site.

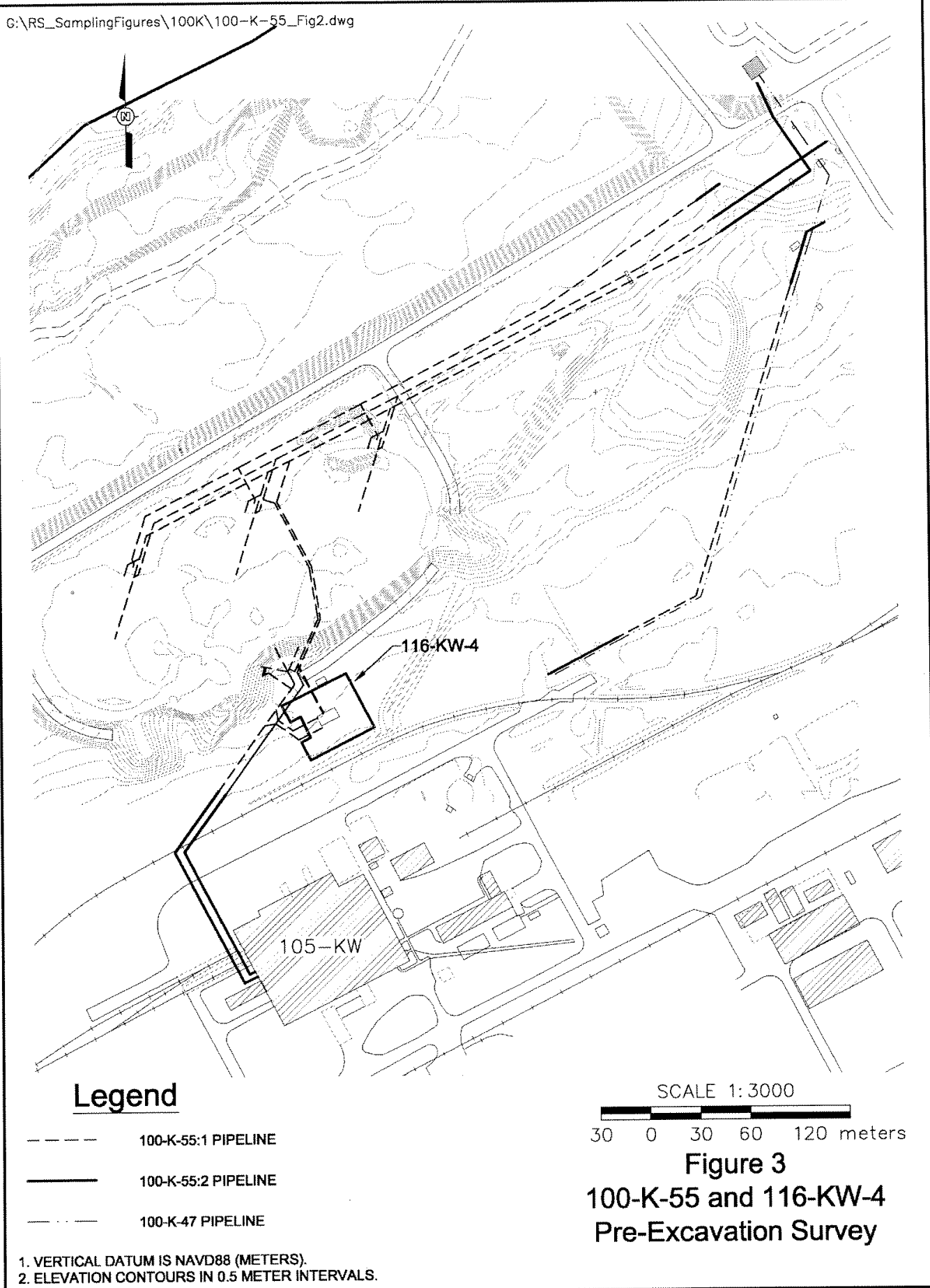


Figure 4. Pre-Remediation Topographic Plan for the Western 100-K-56 Pipelines and 116-KE-5 Site.

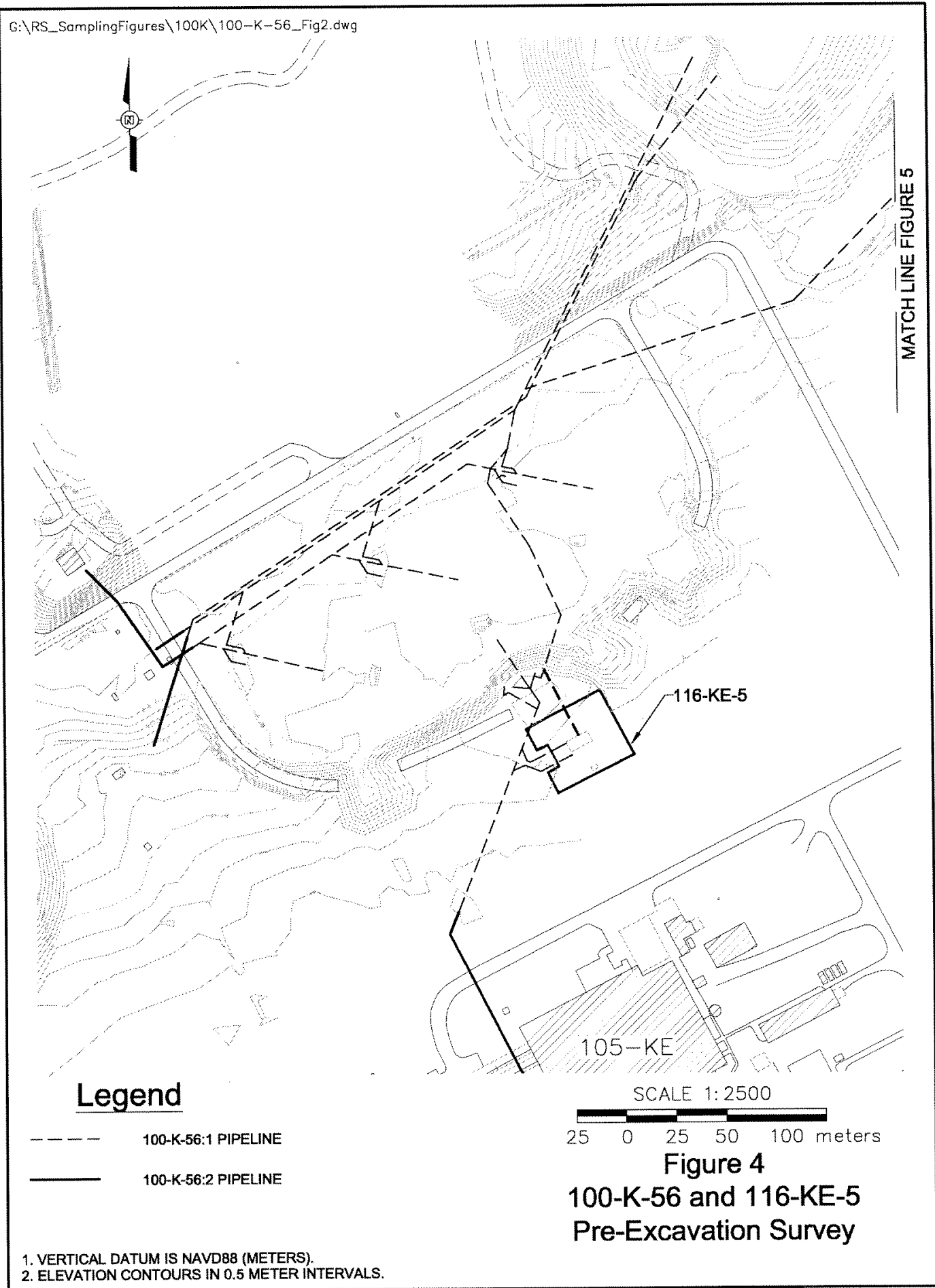


Figure 5. Pre-Remediation Topographic Plan for the Eastern 100-K-56 Pipelines.

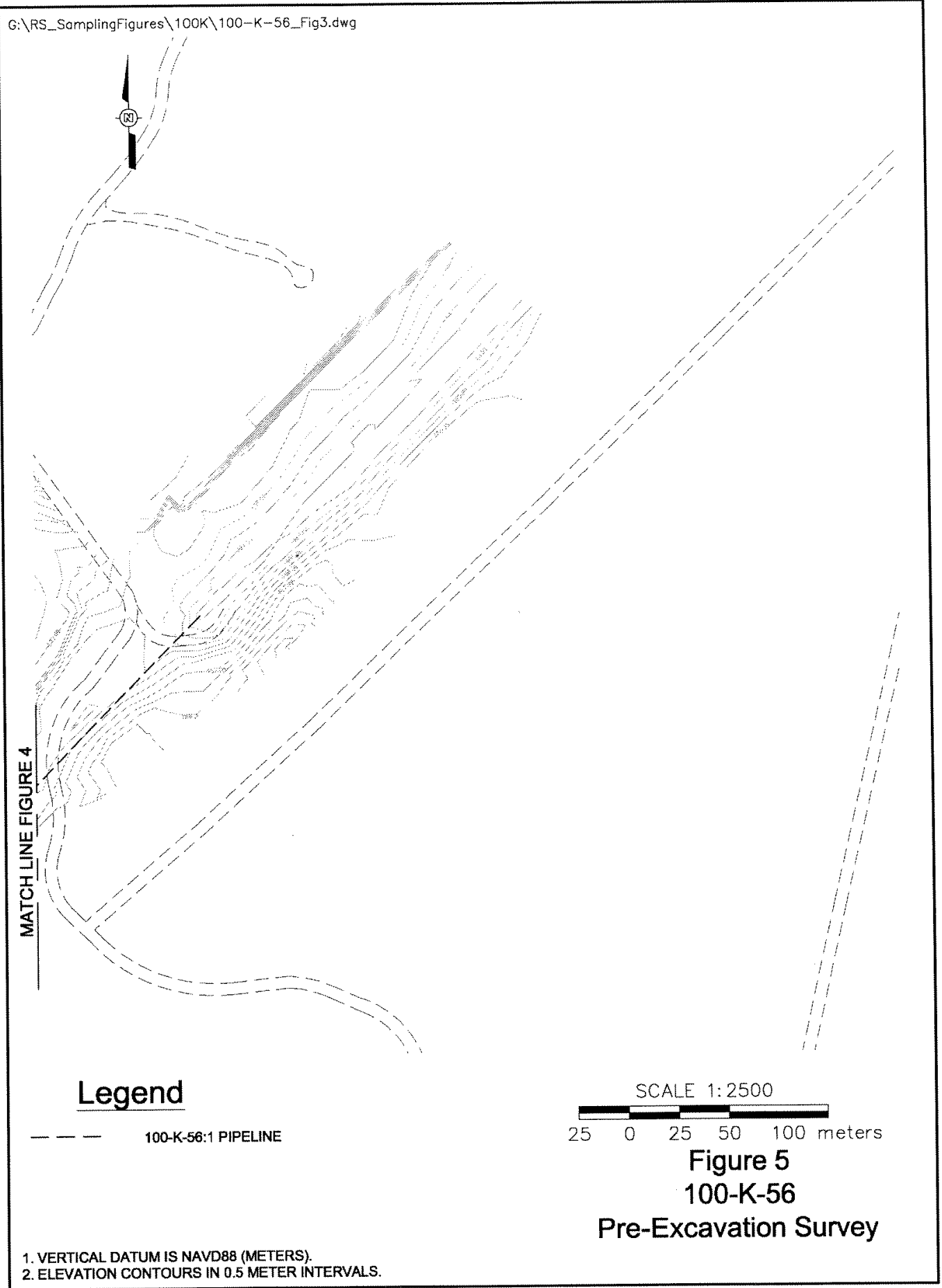


Figure 6. Post-Remediation Topographic Plan for the 100-K-55 Pipelines and 116-KW-4 Site.

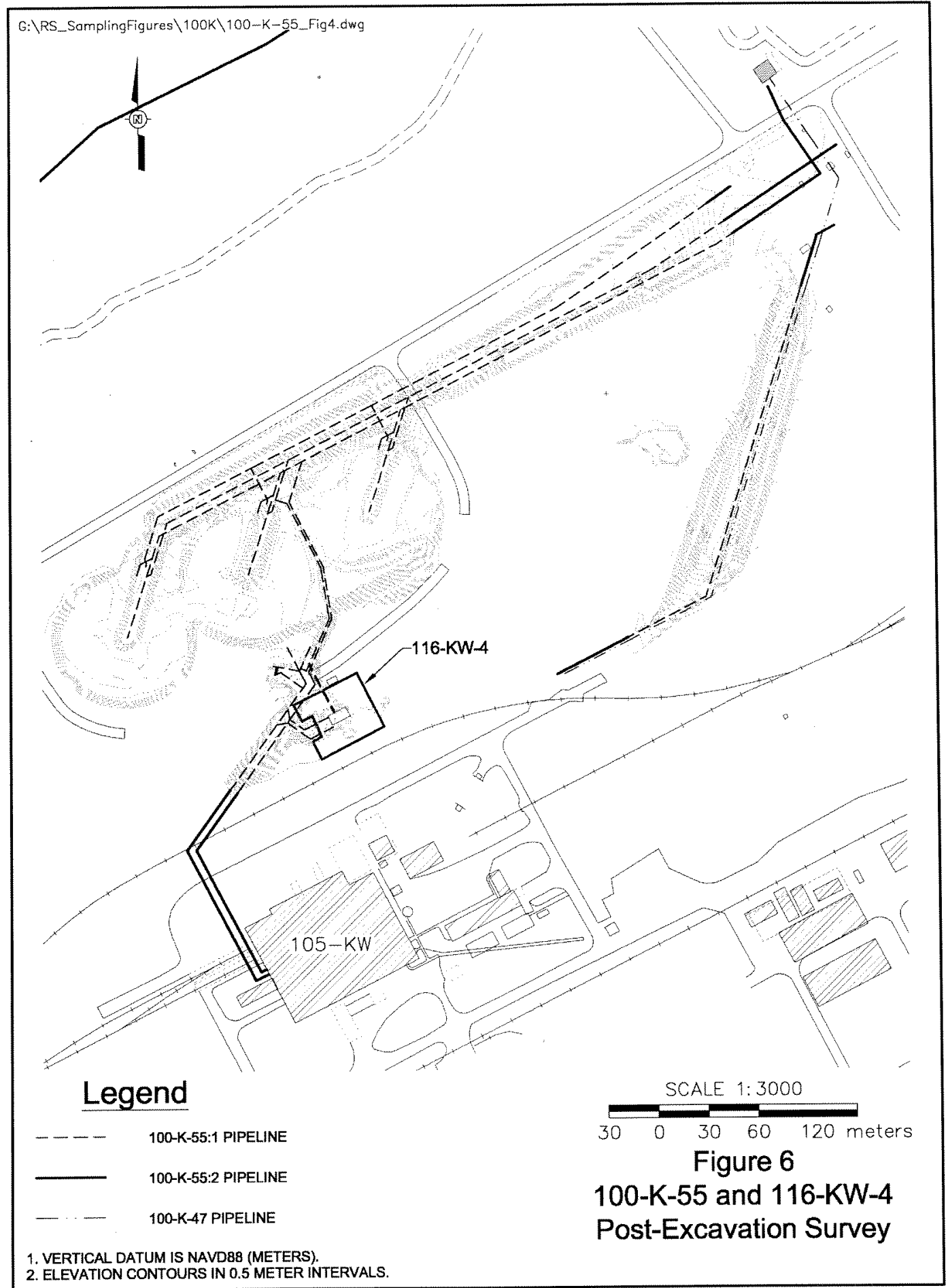


Figure 7. Post-Remediation Topographic Plan for the Western 100-K-56 Pipelines and 116-KE-5 Site.

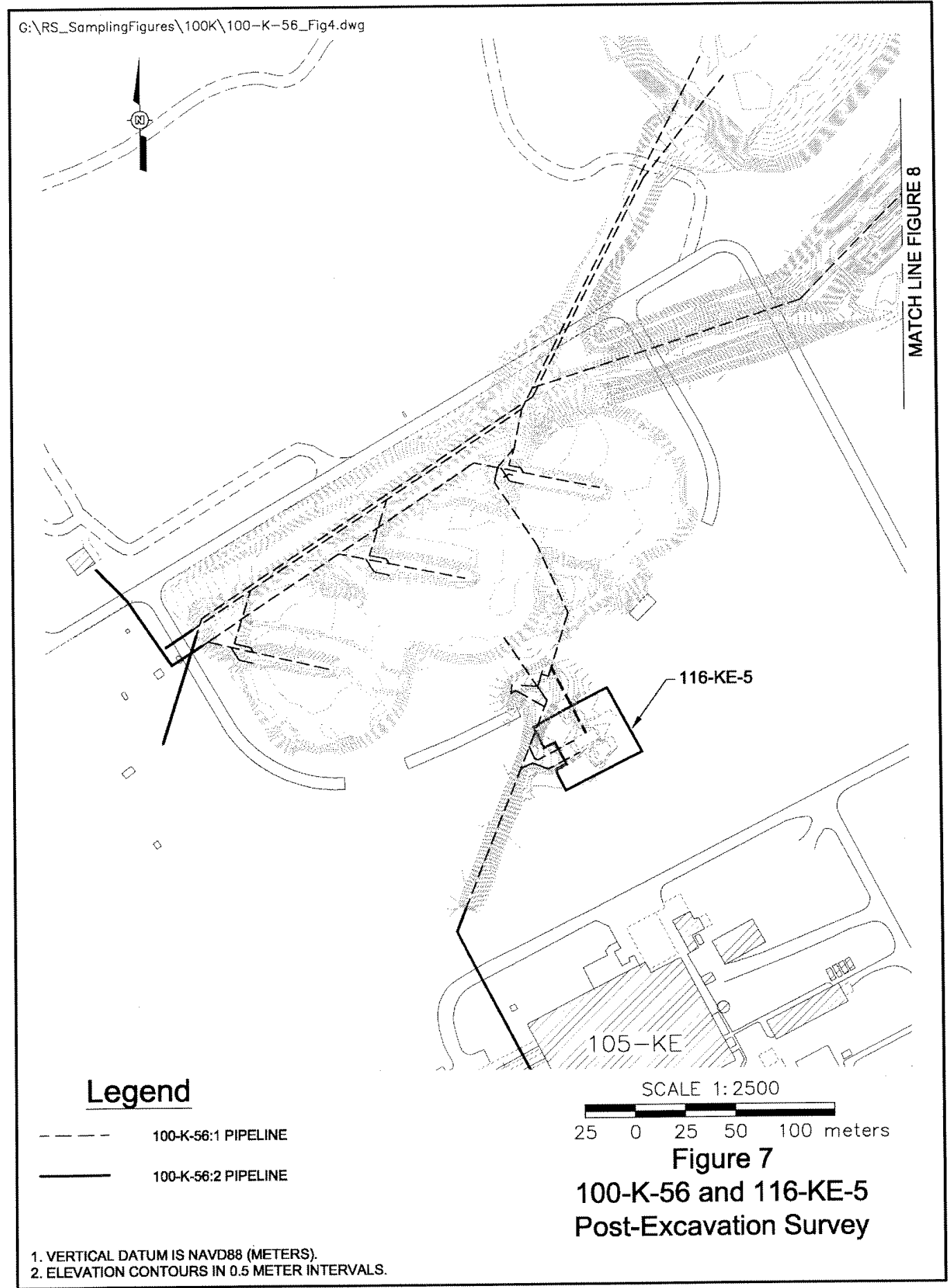


Figure 8. Post-Remediation Topographic Plan for the Eastern 100-K-56:1 Pipelines.

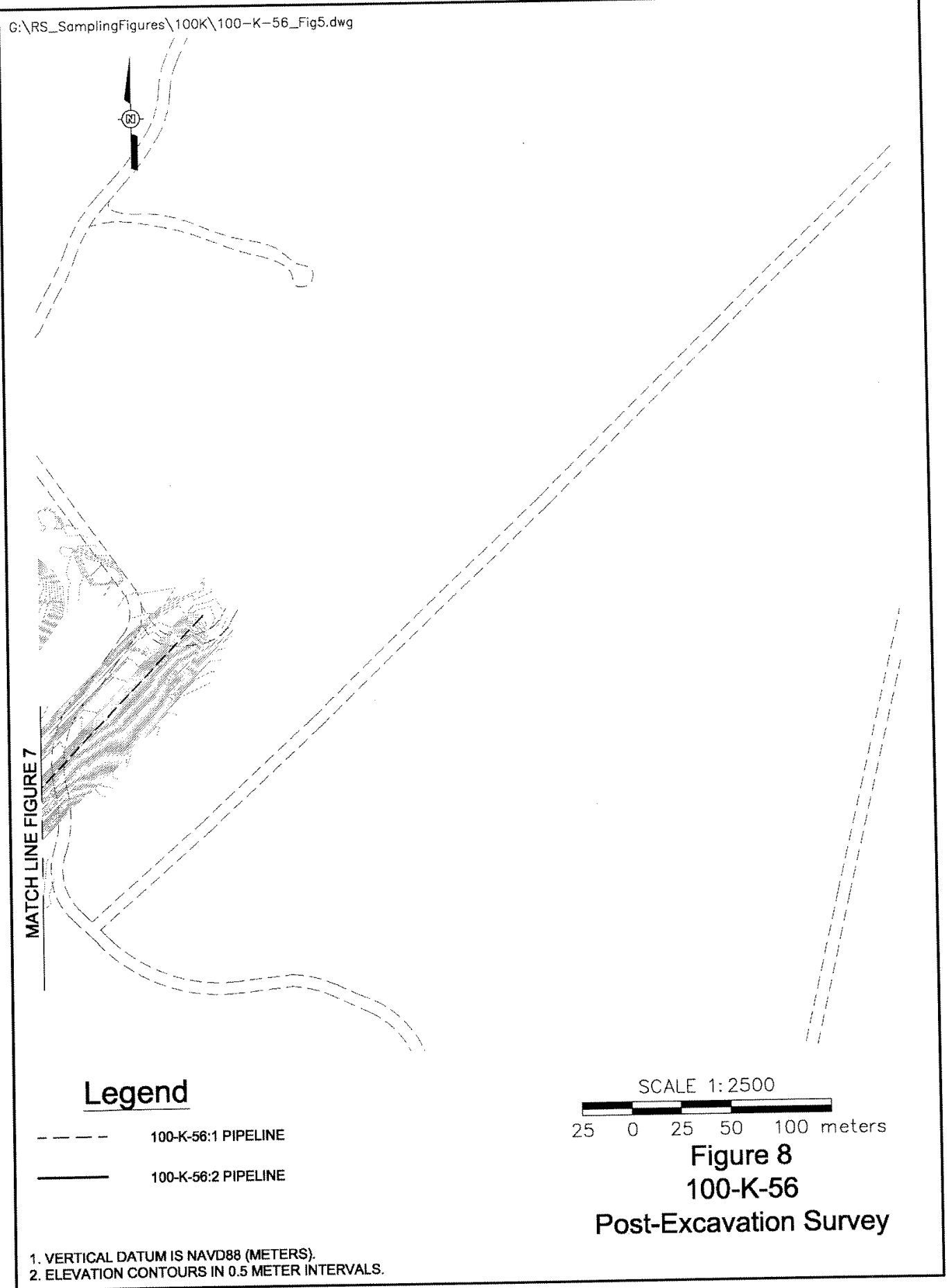


CVP-2005-00006

Rev. 0

\subsection{FIELD SCREENING AND VARIANCE SAMPLING}

Radiological field screening was conducted during the site remedial actions as specified in the SAP (DOE-RL 2001a). Field screening was used to guide the excavation to quickly assess for the presence and level of contamination. Field screening for the sites included using a radiological data mapping system survey, hand-held sodium iodide (Nal) detectors, and gamma energy analyses of grab samples. The radiological mapping survey was performed over more than $50 \%$ of the site excavation surface area. The hand-held $\mathrm{Nal}$ detectors were used to screen excavated waste material and to screen for potential excavation wall and floor hot spots. Gamma energy analyses were used to support waste characterization and to corroborate the radiological mapping survey and hand-held $\mathrm{Nal}$ detector data.

Variance analysis was performed following field screening. The variance analysis quantifies the variability of residual contamination (see calculation brief in Appendix C). This information was used to determine the site-specific number of final cleanup verification samples to be collected. Initial analytical results indicated an area of excessive residual radionuclide contamination in area $\mathrm{E9}$ of the 100-K-56:1 remediation footprint (sample J037K1 at node E9-14). Additional remediation was performed and a second sample (J03JN1) collected at the sampling node pursuant to the SAP (DOE-RL 2005a). The analytical results for the first sample were replaced with those for the second sample for the purposes of variance calculations (Appendix C).

The results of the variance analysis indicated that the number of verification samples to be taken for each shallow zone and overburden decision subunit of the 100-K-55:1 and $100-\mathrm{K}-56: 1$ sites was less than the default number of four specified in the SAP (DOE-RL 2005a); therefore, four final verification samples were collected from each shallow zone and overburden decision subunit. The default number of three (DOE-RL 2005a) verification samples was collected from each deep zone decision subunit.

Variance analysis was not performed separately for the 116-KW-4 and 116-KE-5 sites, as the excavated footprints of these sites were considered within analyses of the 100-K-55:1 and 100-K-56:1 sites, respectively.

\subsection{CLEANUP VERIFICATION SAMPLING AND ANALYSIS}

Final cleanup verification sampling was conducted from January 26, 2005, to June 23, 2005 (BHI 2005b, 2005c), following variance analysis. The final verification samples were submitted to offsite laboratories for analysis using approved U.S. Environmental Protection Agency (EPA) analytical methods as required per the SAP (DOE-RL 2005a). Each verification sample was composed of a composite sample formed by combining soil collected at the required number of randomly selected locations within each sampling area (excluding the quality assurance/quality control samples). 
The division of the 100-K-55:1 and 100-K-56:1 site excavations into decision units (i.e., shallow zone and deep zone) as shown on the sample design figures (Appendix C) is a function of the applicable RAGs. The direct exposure, groundwater protection, and river protection RAGs are applicable to soils within $4.6 \mathrm{~m}(15 \mathrm{ft})$ of the ground surface (i.e., shallow zone and overburden soil). The groundwater protection and river protection RAGs are applicable to soils greater than $4.6 \mathrm{~m}(15 \mathrm{ft})$ below the ground surface (i.e., deep zone).

The 100-K-55:1 and 100-K-56:1 sites consisted of shallow zone, deep zone, and overburden decision units. The shallow zone consisted of the excavation sidewalls and floors that were less than $4.6 \mathrm{~m}$ (15 ft) below ground surface. The deep zone consisted of the portions of the excavation sidewalls and excavation floor that were more than $4.6 \mathrm{~m}(15 \mathrm{ft})$ below ground surface. The shallow zone decision units for the 100-K-55:1 and 100-K-56:1 sites contained six and five decision subunits, respectively, divided into four sampling areas per decision subunit. The deep zone decision units for the 100-K-55:1 and 100-K-56:1 sites contained four and three decision subunits, respectively, divided into three sampling areas per decision subunit. The combined overburden decision unit for the sites contained 12 subunits, divided into four sampling areas per decision subunit. All sampling areas were further divided into 16 sampling nodes each as shown in the sample design methodology and sample location figures presented in the calculation briefs for variance analysis and sample design in Appendix C.

In addition to the statistical verification samples collected, one discrete verification sample was collected in each of the footprints of the 116-KW-4 and 116-KE-5 sites, and analyzed for COPCs associated with these sites. A total of six discrete verification samples were also collected within the footprints of the 116-KW-3 and 116-KE-4 retention basins at the former points of discharge to the $100-\mathrm{K}-55$ and $100-\mathrm{K}-56$ pipelines. These samples were analyzed for the COCs associated with the retention basins. All discrete samples were collected from shallow zone soils (the 116-KW-3, 116-KW-4, 116-KE-4, and 116-KE-5 sites do not contain a deep zone) at locations shown in the figures included with the sample designs in Appendix C.

\subsection{CLEANUP VERIFICATION DATA EVALUATION}

This section presents the evaluation and modeling of the 100-K-55:1, 100-K-56:1, $116-K W-4$, and $116-K E-5$ cleanup verification data for comparison with the data quality criteria and RAGs.

\subsection{DATA QUALITY ASSESSMENT PROCESS}

A data quality assessment (DQA) is performed to compare the verification sampling approach and resulting analytical data with the sampling and data quality requirements specified by the project objectives and performance specifications. 
The DQA for the 100-K-55:1, 100-K-56:1, 116-KW-4, and 116-KE-5 sites determined that the data are of the right type, quality, and quantity to support site verification decisions within specified error tolerances. All analytical data were found to be acceptable for decision-making purposes. The evaluation also verified that the sample design was sufficient to support clean site verification. The cleanup verification sample analytical data are stored in the Hanford Environmental Information System and are summarized in Appendix A. The detailed DQA is presented in Appendix B.

\subsection{CONTAMINANTS OF CONCERN 95\% UPPER CONFIDENCE LIMIT}

The primary statistical calculation to support cleanup verification is the $95 \%$ upper confidence limit (UCL) on the arithmetic mean of the data. The 95\% UCL values for each COC are computed for each decision unit (e.g., for the shallow and deep zones for each site, as appropriate). Prior to calculating the $95 \%$ UCL, the individual sample results are reviewed and, as appropriate, adjusted per the SAP (DOE-RL 2005a). This process is summarized below.

Verification sampling summary statistics (95\% UCL values) for the 100-K-55:1 and 100-K-56:1 pipelines are listed in Table 3. Individual sample cleanup verification results are presented in Appendix A.

Table 3. $100-K-55: 1$ and $100-K-56: 1$ Cleanup Verification Data Set.

\begin{tabular}{|c|c|c|c|c|c|c|c|}
\hline \multirow{2}{*}{ cocs } & \multicolumn{3}{|c|}{$\begin{array}{l}95 \% \text { UCL Statistical Values } \\
(\mathrm{pCl} / \mathrm{g})\end{array}$} & \multirow{2}{*}{$\begin{array}{l}\text { Hanford Site } \\
\text { Background } \\
\text { (pCi/g) }\end{array}$} & \multicolumn{3}{|c|}{$\begin{array}{l}\text { Cleanup Verification Data Set }^{c} \\
(\mathrm{pCli} / \mathrm{g})\end{array}$} \\
\hline & $\begin{array}{l}\text { Shallow } \\
\text { Zone }\end{array}$ & $\begin{array}{l}\text { Deep } \\
\text { Zone }\end{array}$ & Overburden $^{a}$ & & $\begin{array}{l}\text { Shallow } \\
\text { Zone }\end{array}$ & $\begin{array}{l}\text { Deep } \\
\text { Zone }\end{array}$ & Overburden \\
\hline \multicolumn{8}{|c|}{$100-K-55: 1^{d}$} \\
\hline Cesium-137 & 0.27 & 0.82 & 0.30 & 1.1 & 0.27 & 0.82 & $0(<B G)$ \\
\hline Europium-152 & 0.73 & 0.74 & 0.30 & NA & 0.73 & 0.74 & 0.30 \\
\hline Europium-154 & 0.12 & 0.12 & 0.086 & 0.033 & 0.12 & 0.12 & 0.053 \\
\hline \multicolumn{8}{|c|}{$100-K-56: 1^{d}$} \\
\hline Cesium-137 & 0.61 & 1.32 & 0.30 & 1.1 & 0.61 & 1.32 & $0(<B G)$ \\
\hline Europium-152 & 1.7 & 13.7 & 0.30 & NA & 1.7 & 13.7 & 0.30 \\
\hline Europium-154 & 0.14 & 1.87 & 0.086 & 0.033 & 0.14 & 1.87 & 0.053 \\
\hline
\end{tabular}

${ }^{2}$ Overburden material from the $100-\mathrm{K}-55: 1$ and $100-\mathrm{K}-56: 1$ pipelines was considered as one decision unit.

${ }^{b}$ Represents the 90th percentile of the lognormal distribution (DOE-RL 1996).

${ }^{c}$ For overburden, anthropogenic background (DOE-RL 1996) and naturally occurring background is subtracted from all radionuclides. For other decision units (e.g., shallow zone and deep zone), only naturally occurring background (uranium) is subtracted. Refer to the $95 \%$ UCL calculation brief in Appendix C for additional details on determination of statistical values.

'Laboratory data, including the minimum detectable activities for the individual cleanup verification samples, are included in Appendix A and the 95\% UCL calculation briefs in Appendix C.

$B G=$ background

$\mathrm{COC}=$ contaminant of concern

$\mathrm{NA}=$ not applicable

$\mathrm{UCL}=$ upper confidence limit 
For radionuclides, the laboratory-reported value is used in the calculation of the $95 \%$ UCL. In cases where the laboratory does not report a value for data qualified with a "U" (i.e., less than the detection limit), one-half of the minimum detectable activity is used in the calculation of the $95 \%$ UCL.

Statistical calculations are presented in the 100-K-55:1 95\% UCL calculation and the 100-K-56:1 95\% UCL calculation (Appendix C), with results shown in Table 3. The columns on the left side of Table 3 are the COCs and the $95 \%$ statistical values before subtraction of background. The fifth column of Table 3 presents the background where values exist, and the last three columns present the statistical values adjusted for background, if appropriate, which becomes the cleanup verification data set used for RESidual RADioactivity (RESRAD) modeling.

Additional cleanup verification data collected at the points of discharge from the $116-\mathrm{KW}-3$ and 116-KE-4 retention basins is provided in Table 4 . These locations are considered focused, worst-case locations, and, as such, are evaluated as discrete points separate from the statistical data set. The first two columns of Table 4 present the COCs for the retention basins (which are inclusive of the effluent pipeline COCs) and maximum analytical results, and the final column presents the background concentration where values exist.

Verification sampling of the 116-KW-4 and 116-KE-5 sites was based on a focused, rather than statistical, sampling design. As such, statistical analysis (e.g., calculation of a $95 \%$ UCL value) is inappropriate, and evaluation of the data sets is based on the maximum detected concentration of each COPC as reported in Table 5. The first two columns of Table 5 present the COPCs and maximum analytical results, and the final column presents the background concentration where values exist. Reported results are not adjusted for background for any of the contaminants listed, as comparison against background is considered in evaluation of RAG attainment in Section 5.0.

The results of discrete sampling at the 100-K-55:1 and 100-K-56:1 sites within the footprints of the former $116-\mathrm{KW}-3$ and $116-\mathrm{KE}-4$ retention basins are provided in Appendix A. 
Table 4. Maximum Concentrations at the Former 116-KW-3 and 116-KE-4 Retention Basin Discharge Points.

\begin{tabular}{|c|c|c|}
\hline cocs & $\begin{array}{l}\text { Maximum Values } \\
(\mathrm{pCi} / \mathrm{g})\end{array}$ & $\begin{array}{l}\text { Hanford Site Background } \\
\qquad(\mathrm{pCi} / \mathrm{g})\end{array}$ \\
\hline \multicolumn{3}{|c|}{ 116-KW-3 Radionuclides ${ }^{b}$} \\
\hline Cesium-137 & 0.61 & NA \\
\hline Cobalt-60 & 0.11 & NA \\
\hline Europium-152 & 1.3 & NA \\
\hline Europium-154 & 0.19 & NA \\
\hline Plutonium-239/240 & ND & NA \\
\hline Strontium-90 & 0.366 & NA \\
\hline Uranium-233/234 & 0.70 & 1.10 \\
\hline Uranium-235 & 0.094 & 0.11 \\
\hline \multicolumn{3}{|c|}{$116-K W-3$ Nonradionuclides ${ }^{b}$} \\
\hline Hexavalent chromium & ND & NA \\
\hline \multicolumn{3}{|c|}{ 116-KE-4 Radionuclides ${ }^{b}$} \\
\hline Cesium-137 & 0.31 & NA \\
\hline Cobalt-60 & 0.15 & NA \\
\hline Europium-152 & 3.11 & NA \\
\hline Europium-154 & 0.33 & NA \\
\hline Plutonium-239/240 & ND & NA \\
\hline Strontium-90 & 0.496 & NA \\
\hline Uranium-233/234 & 0.454 & 1.10 \\
\hline Uranium-235 & 0.027 & 0.11 \\
\hline \multicolumn{3}{|c|}{ 116-KE-4 Nonradionuclides ${ }^{b}$} \\
\hline Hexavalent chromium & 0.94 & NA \\
\hline \multicolumn{3}{|c|}{$\begin{array}{l}\text { a Represents the 90th percentile of the lognormal distribution (DOE-RL 1996). } \\
\text { b Laboratory data, including the practical quantitation limits for the individual cleanup } \\
\text { verification samples, are included in Appendix A. } \\
\text { COC = contaminant of potential concern } \\
\text { NA = not applicable } \\
\text { ND = not detected }\end{array}$} \\
\hline
\end{tabular}


Table 5. 116-KW-4 and 116-KE-5 Cleanup Verification Data Set.

\begin{tabular}{|l|c|c|}
\hline \multicolumn{1}{|c|}{ COPCs } & $\begin{array}{c}\text { Maximum Values } \\
\text { (mg/kg) }\end{array}$ & $\begin{array}{c}\text { Hanford Site Background } \\
\text { (mg/kg) }\end{array}$ \\
\hline & $\mathbf{1 1 6 - K W - 4 ^ { b }}$ \\
\hline Ethylene glycol & $\mathrm{ND}$ & $\mathrm{NA}$ \\
\hline Arsenic & 2.6 & $20^{\mathrm{b}}$ \\
\hline Barium & 59.3 & 132 \\
\hline Cadmium & $\mathrm{ND}$ & $0.81^{\mathrm{d}}$ \\
\hline Chromium (total) & 12.1 & 18.5 \\
\hline Hexavalent chromium & 0.24 & $\mathrm{NA}$ \\
\hline Lead & 4.5 & 10.2 \\
\hline Mercury & 0.02 & 0.33 \\
\hline \multicolumn{2}{|c|}{$116-K E-5^{b}$} \\
\hline Ethylene glycol & 59 & $\mathrm{NA}$ \\
\hline Arsenic & 3.7 & $20^{\mathrm{C}}$ \\
\hline Barium & 100 & 132 \\
\hline Cadmium & ND & $0.81^{\mathrm{d}}$ \\
\hline Chromium (total) & 30 & 18.5 \\
\hline Hexavalent chromium & 0.33 & $\mathrm{NA}$ \\
\hline Lead & 11.3 & 10.2 \\
\hline Mercury & 0.10 & 0.33 \\
\hline
\end{tabular}

\subsection{SITE-SPECIFIC CLEANUP VERIFICATION MODEL}

The statistical values summarized in Table 3 were evaluated and used to develop sitespecific cleanup verification models. The 100-K-55:1 and 100-K-56:1 site cleanup verification models comprise two depth intervals: (1) the shallow zone and overburden and (2) the deep zone. The site-specific cleanup verification model for all COCs assumes the worst case described in the RDR/RAWP (DOE-RL 2005b), where the deep zone statistical concentrations continue at the same concentrations to groundwater. Schematic cross sections of this site-specific cleanup verification model are included in the RESRAD calculations in Appendix C. The elements of the RESRAD modeling are described in Section 4.4. 


\subsection{RESRAD MODELING}

The individual radionuclide cleanup verification statistical values (Table 3 ) were entered into the RESRAD computer code, Version 6.22 (ANL 2004), to estimate the residential dose rate and the impact on groundwater and the Columbia River from residual COC concentrations. The direct radiation exposure dose rate to the resident living in his or her basement (rural-residential scenario) was conservatively estimated by substituting (for analysis purposes) a case where the resident is standing on level ground with the soil containing concentrations representative of residual (i.e., post-cleanup) shallow zone soils. This is conservative because it ignores the potential shielding effects of concrete basement walls and any clean backfill between residual soils and the basement walls.

The RESRAD modeling methodologies, results, input values, and the site-specific cleanup verification model are included in the RESRAD calculation brief (Appendix C). The drinking water dose rate calculations based on the RESRAD estimated groundwater radionuclide concentrations are shown in the comparison to drinking water standards calculation brief (Appendix C). Specific results from the calculations are discussed as part the RAG-attainment evaluation (Section 5.0).

\subsection{EVALUATION OF REMEDIAL ACTION GOAL ATTAINMENT}

This section demonstrates that remedial actions at the 100-K-55:1, 100-K-56:1, 116-KW-4, and 116-KE-5 sites have achieved the applicable RAGs. Sections 5.1, 5.2, and 5.3 address attainment of direct exposure RAGs, groundwater protection RAGs, and Columbia River protection RAGs, respectively. Section 5.4 documents application of the Washington Administrative Code (WAC) 173-340-740(7)(e) three-part test, which is required for nonradionuclide COCs only.

\subsection{DIRECT EXPOSURE SOIL REMEDIAL ACTION GOALS ATTAINED}

\subsubsection{Radionuclides}

The results of the combined RESRAD dose rate estimates for the 100-K-55:1 site shallow and deep zone all-pathways scenarios are presented in Figure 9. The results of the combined RESRAD dose rate estimates for the 100-K-56:1 site shallow and deep zone all-pathways scenarios are presented in Figure 10. The results of the RESRAD dose rate estimate for overburden material under all-pathways scenarios are presented in Figure 11. No radionuclide COCs were identified for the 116-KW-4 and $116-\mathrm{KE}-5$ sites separate from the associated pipeline sites. The dose rates presented represent the dose contributions from soils at relevant time periods. 
Figure 9. Combined Shallow and Deep Zone Dose Rate Estimates for the 100-K-55:1 Site (All Radionuclides, All Pathways).

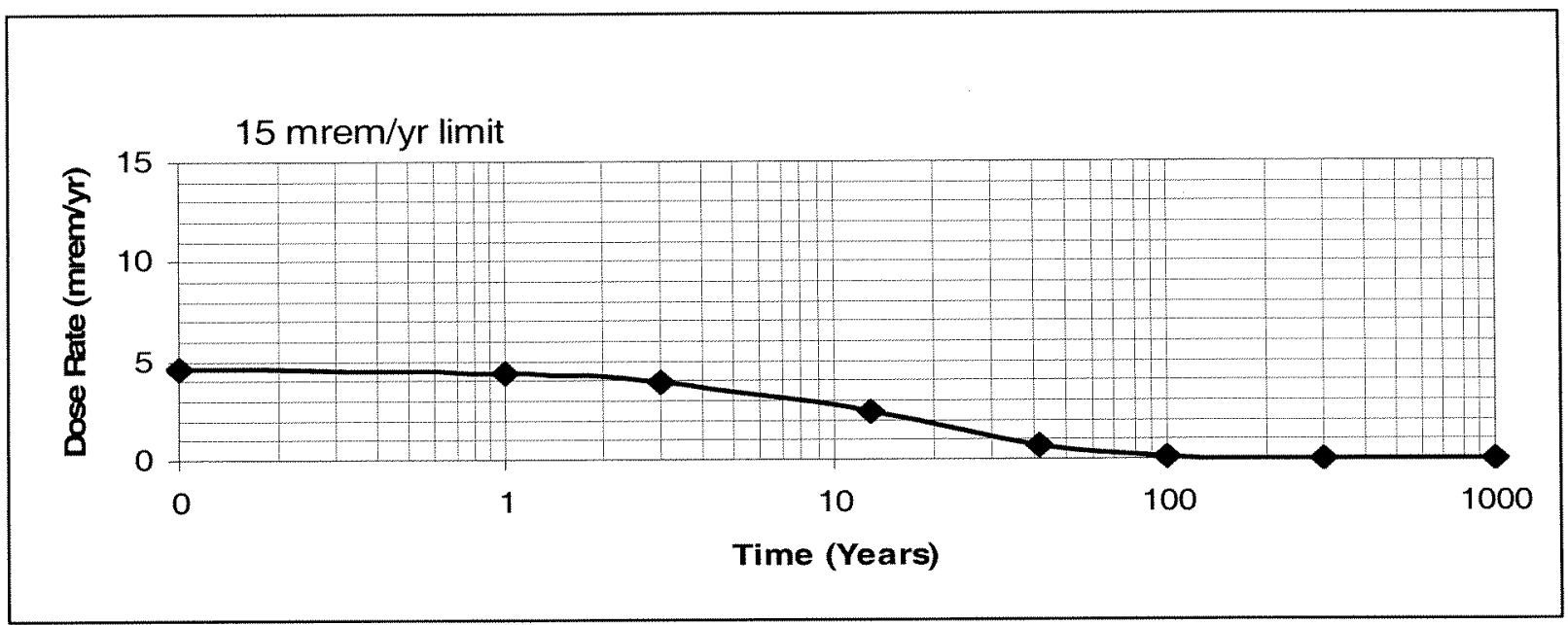

Figure 10. Combined Shallow and Deep Zone Dose Rate Estimates for the 100-K-56:1 Site (All Radionuclides, All Pathways).

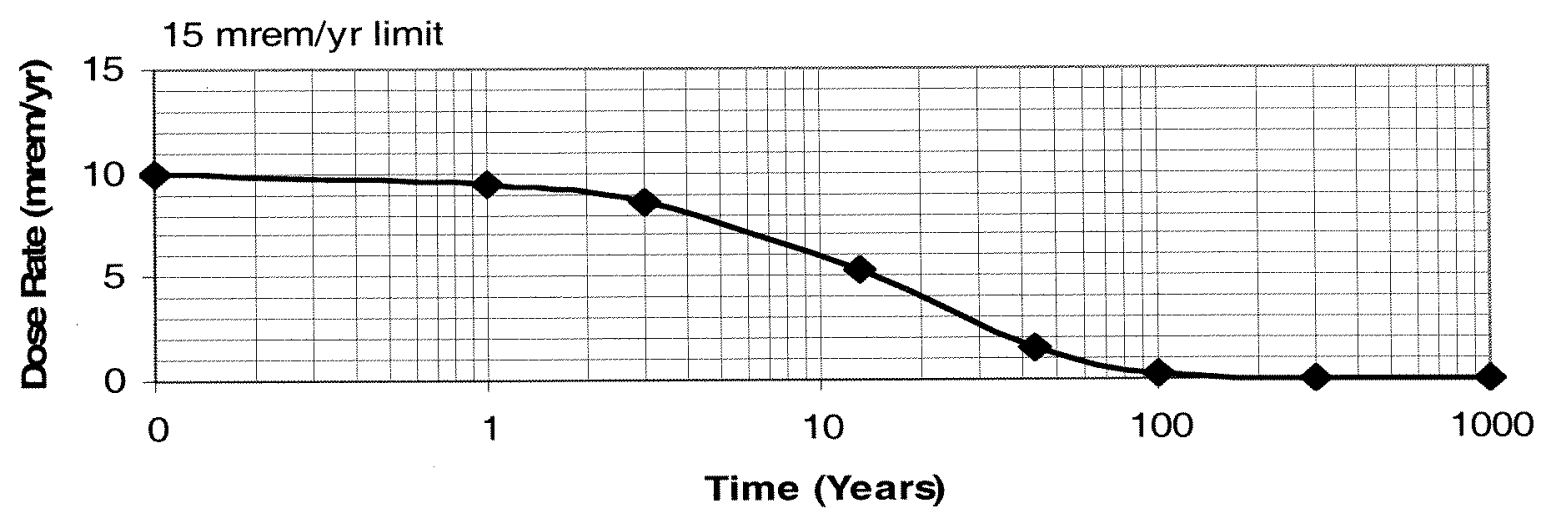


Figure 11. Overburden Dose Rate Estimates for the 100-K-55:1 and 100-K-56:1 Sites (All Radionuclides, All Pathways).

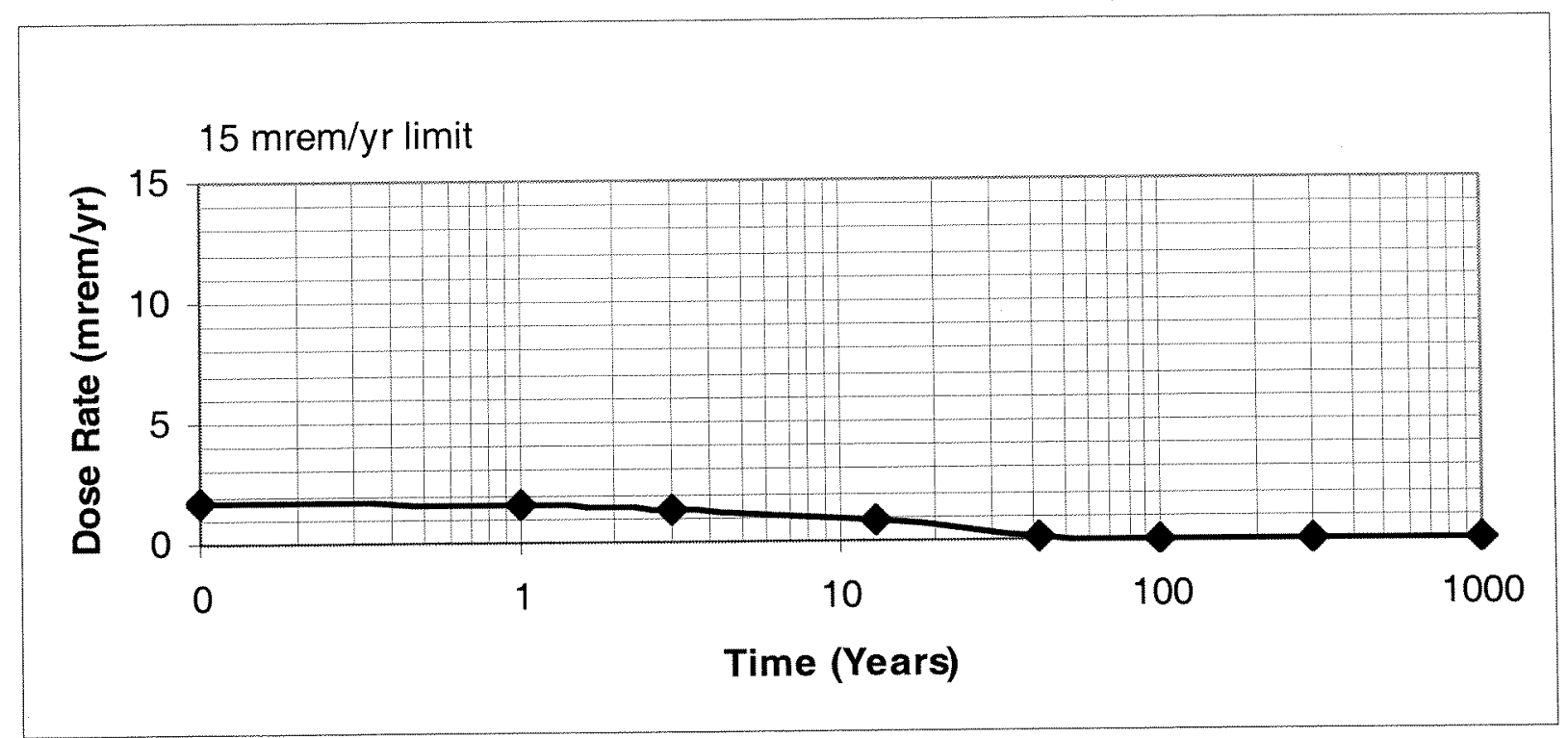

The dose rate for the 100-K-55:1 site is largest at present (year 2005), at $4.59 \mathrm{mrem} / \mathrm{yr}$, decreasing to $9.77 \times 10^{-11} \mathrm{mrem} / \mathrm{yr}$ in 1,000 years for the shallow zone and deep zone. The dose rate for the 100-K-56:1 site is also largest at present (year 2005), at $9.95 \mathrm{mrem} / \mathrm{yr}$, decreasing to $3.90 \times 10^{-10} \mathrm{mrem} / \mathrm{yr}$ in 1,000 years for the shallow and deep zones combined. The estimated dose rate in the year 2018 is $2.41 \mathrm{mrem} / \mathrm{yr}$ for the $100-\mathrm{K}-55: 1$ site and $5.34 \mathrm{mrem} / \mathrm{yr}$ for the $100-\mathrm{K}-56: 1$ site. The 2018 date corresponds to the original 30-year site cleanup schedule of the Hanford Federal Facility Agreement and Consent Order (Ecology et al. 1989). The dose rate for the combined overburden soils from the 100-K-55:1 and 100-K-56:1 sites is largest at present (year 2005), at $1.66 \mathrm{mrem} / \mathrm{yr}$, decreasing to $0.804 \mathrm{mrem} / \mathrm{yr}$ in 2018 and to $5.39 \times 10^{-16} \mathrm{mrem} / \mathrm{yr}$ in 1,000 years. All dose rate estimates are less than the $15 \mathrm{mrem} / \mathrm{yr}$ RAG. The RESRAD computations are presented in detail in the RESRAD calculation briefs summarized in Appendix $C$.

Residual radionuclide concentrations detected in the discrete samples taken in the footprints of the former 116-KW-3 and 116-KE-4 retention basins are consistent with the residual concentrations reported in the CVPs for those sites (BHI 2004, 2005d).

\subsubsection{Nonradionuclides}

5.1.2.1 Direct Comparison to RAGs. Ethylene glycol, the primary COC for the 116-KW-4 and 116-KE-5 heat recovery stations, was not detected in the discrete sample taken from the 116-KW-4 site and was detected at a concentration of $59 \mathrm{mg} / \mathrm{kg}$ in the discrete sample taken from the 116-KE-5 site. This residual concentration meets the RAG of $160,000 \mathrm{mg} / \mathrm{kg}$. In addition, the discrete samples collected from these sites were also analyzed for arsenic, barium, cadmium, total chromium, hexavalent 
chromium, mercury, and lead. Detected concentrations of all of these metals (shown in Table 5) were below background levels or direct exposure RAGs.

No nonradionuclide COCs were identified for the 100-K-55:1 or 100-K-56:1 pipelines, but hexavalent chromium concentrations were evaluated at discrete locations at the points of discharge from the 116-KW-3 and 116-KE-4 retention basins. As shown in Table 4, hexavalent chromium was not detected in the samples collected at the former discharge points from the 116-KW-3 retention basins. Concentrations of hexavalent chromium detected at the former discharge points of the $116-\mathrm{KW}-4$ retention basins are below the direct exposure RAG and consistent with the residual concentrations for the entire basin footprint (BHI 2005d).

5.1.2.2 Noncarcinogenic Hazard Quotient RAG Attained. For noncarcinogenic COCs, WAC 173-340-740(5)(a) and (b) specify the evaluation of the hazard quotient, which is given as daily intake divided by a reference dose (DOE-RL 2005a). No nonradionuclide COCs were identified for the 100-K-55:1 or 100-K-56:1 pipelines; therefore, requirements for the determination of hazard quotient values are not applicable. The hazard quotient values for the $116-\mathrm{KW}-4$ and $116-\mathrm{KE}-5$ sites were based on the concentrations of metals detected above background levels and ethylene glycol reported in Table 5. The only constituent detected above background for the $116-\mathrm{KW}-4$ site was hexavalent chromium; a hazard quotient of $1.0 \times 10^{-3}$ was determined for residual concentrations of this constituent (Appendix $\mathrm{C}$ ). A cumulative hazard quotient of $3.4 \times 10^{-2}$ was calculated for the $116-\mathrm{KE}-5$ site based on the residual concentrations of ethylene glycol, hexavalent chromium, total chromium, and lead (Appendix C). The hazard quotients for both of these sites are below 1.0.

5.1.2.3 Carcinogenic Risk RAG Attained. For individual nonradionuclide carcinogenic COCs, the WAC 173-340-750(3) Method B cleanup limits are based on an incremental cancer risk of $1 \times 10^{-6}$. The cumulative excess cancer risk for all nonradionuclide carcinogenic COCs must be less than $1 \times 10^{-5}$ (EPA et al. 1998). No nonradionuclide COCs were identified for the $100-K-55: 1$ or $100-K-56: 1$ pipelines; therefore, requirements for the determination of excess risk values are not applicable. The only carcinogenic constituent detected at the $116-\mathrm{KW}-4$ and $116-\mathrm{KE}-5$ sites was hexavalent chromium, which contributes to excess risk values of $1.1 \times 10^{-7}$ and $1.6 \times 10^{-7}$ for these sites, respectively (Appendix $\mathrm{C}$ ). These values are below the risk limit for individual constituents $\left(1 \times 10^{-6}\right)$ and the cumulative excess cancer RAG of $1 \times 10^{-5}$. 


\subsection{GROUNDWATER REMEDIAL ACTION GOALS ATTAINED}

\subsubsection{Radionuclides}

The estimated groundwater concentrations for all of the radionuclide COCs contributed by the 100-K-55:1 and 100-K-56:1 site soils are shown in the RESRAD calculation briefs (Appendix C). Table 6 shows the total peak concentration predicted for each radionuclide $\mathrm{COC}$ and provides the individual RAGs for comparison. No COC is predicted to exceed the RAGs; therefore, the RAGs are attained. No radionuclide COCs were identified for the $116-\mathrm{KW}-4$ and 116-KE-5 sites.

Table 6. Estimated Peak Radionuclide Groundwater Concentrations Compared to Remedial Action Goals.

\begin{tabular}{|l|c|c|c|}
\hline \multicolumn{1}{|c|}{ Radionuclide } & $\begin{array}{c}\text { Peak Concentration } \\
\text { (pCi/L) }\end{array}$ & $\begin{array}{c}\text { RAG } \\
\text { (pCi/L) }\end{array}$ & $\begin{array}{c}\text { RAGS Attained? } \\
\text { (Yes/No) }\end{array}$ \\
\hline \multicolumn{5}{|c|}{ 100-K-55:1 } \\
\hline Cesium-137 & $4.49 \times 10^{-2}$ & $60^{\mathrm{a}}$ & Yes \\
\hline Europium-152 & $0^{\mathrm{b}}$ & $200^{\mathrm{a}}$ & Yes \\
\hline Europium-154 & $0^{\mathrm{b}}$ & $60^{\mathrm{a}}$ & Yes \\
\hline \multicolumn{5}{|c|}{$100-K-56: 1$} \\
\hline Cesium-137 & $5.41 \times 10^{-1}$ & $60^{\mathrm{a}}$ & Yes \\
\hline Europium-152 & $0^{\mathrm{b}}$ & $200^{\mathrm{a}}$ & Yes \\
\hline Europium-154 & $0^{\mathrm{b}}$ & $60^{\mathrm{a}}$ & \\
\hline
\end{tabular}

${ }^{\mathrm{a}}$ Lookup value corresponding to a dose rate of $4 \mathrm{mrem} / \mathrm{yr}$.

${ }^{\mathrm{D}}$ These radionuclides are not predicted to reach groundwater in 1,000 years.

$R A G=$ remedial action goal

Figures 12 and 13 show individual organ dose rates for beta- and gamma-emitting radionuclides predicted over 1,000 years as calculated in the drinking water standards calculation briefs (Appendix C) for the 100-K-55:1 and 100-K-56:1 sites, respectively. None of the organ dose rates are predicted to exceed the $4 \mathrm{mrem} / \mathrm{yr}$ standard over 1,000 years. 
Figure 12. Dose Rates to Organs from Groundwater Impacted by the 100-K-55:1 Site.

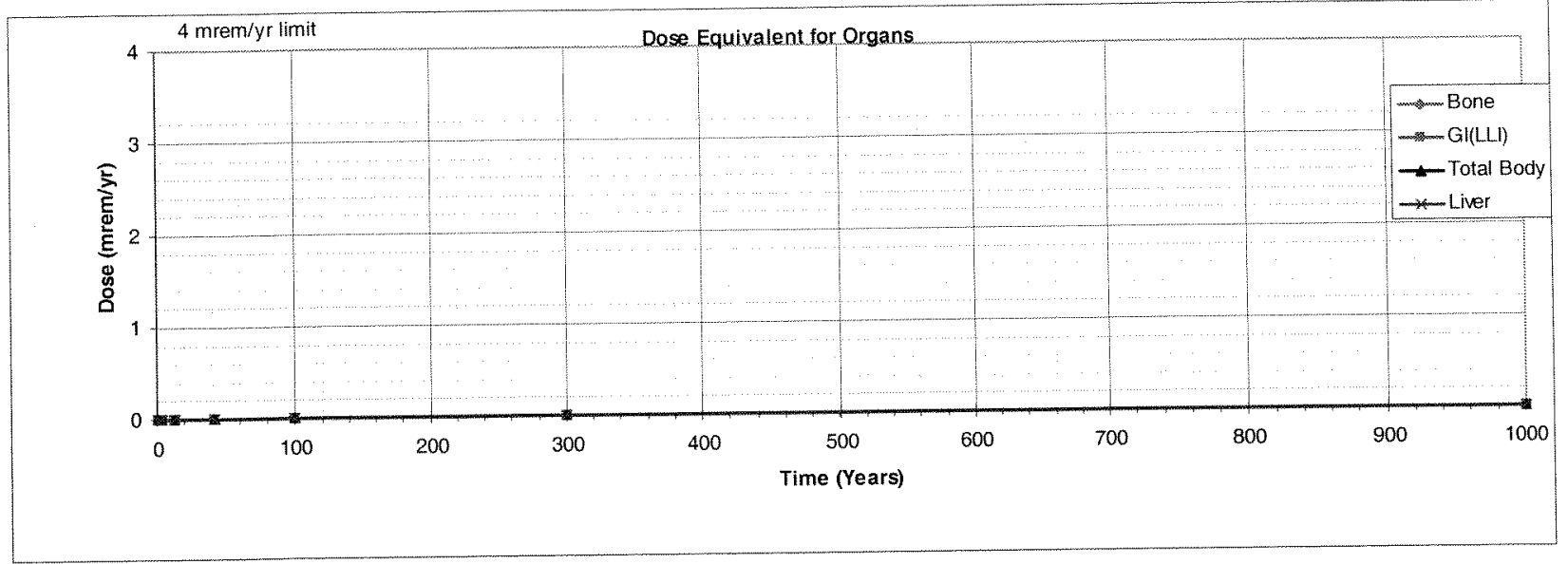

Figure 13. Dose Rates to Organs from Groundwater Impacted by the 100-K-56:1 Site.

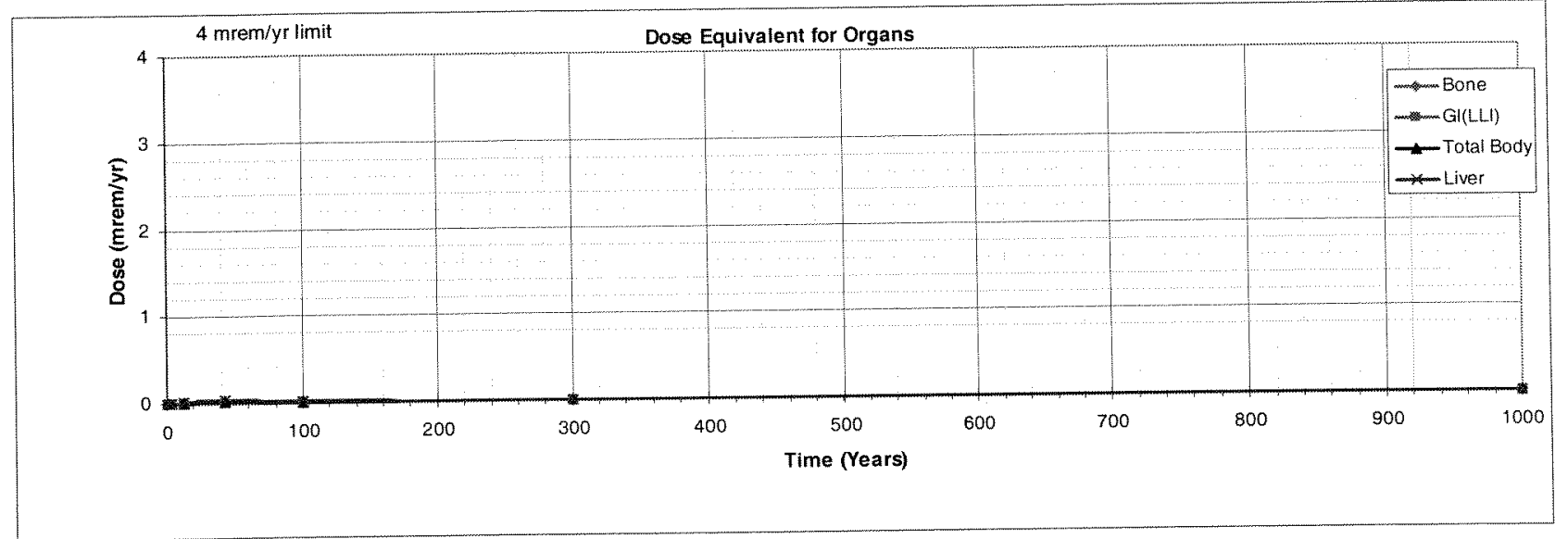

\subsubsection{Nonradionuclides}

Ethylene glycol, the primary COC for the $116-\mathrm{KW}-4$ and $116-\mathrm{KE}-5$ heat recovery stations, was not detected in the discrete sample taken from the $116-\mathrm{KW}-4$ site and was detected at a concentration of $59 \mathrm{mg} / \mathrm{kg}$ in the discrete sample taken from the $116-\mathrm{KE}-5$ site, achieving the $3,200 \mathrm{mg} / \mathrm{kg}$ soil RAG for groundwater protection. Detected concentrations of metals at the two sites (Table 5) were below background levels or soil RAGs for the protection of groundwater (Table 2) with the exception of total chromium and lead concentrations at the $116-\mathrm{KE}-5$ site. Residual concentrations of these metals slightly exceed soil RAGs for the protection of groundwater. However, based on the soil-partitioning coefficient $\left(K_{d}\right)$ values for these metals and the results of the 100 Area Analogous Sites RESRAD Calculations (BHI 2005a), these metals will not reach groundwater within a 1,000-year time frame. 
No nonradionuclide COCs were identified for the 100-K-55:1 or 100-K-56:1 pipelines, but hexavalent chromium concentrations were evaluated at discrete locations at the points of discharge from the $116-\mathrm{KW}-3$ and $116-\mathrm{KE}-4$ retention basins. As shown in Table 4, hexavalent chromium was not detected in the samples collected at the former discharge points from the $116-\mathrm{KW}-3$ retention basins. Concentrations of hexavalent chromium detected at the former discharge points of the $116-\mathrm{KW}-4$ retention basins are below the soil RAG for groundwater protection and consistent with the residual concentrations for the entire basin footprint (BHI 2005d).

\subsection{COLUMBIA RIVER REMEDIAL ACTION GOALS ATTAINED}

\subsubsection{Radionuclides}

The river protection RAGs for radionuclides are identical to the groundwater protection RAGs. The RESRAD modeling results were compared to the groundwater protection RAGs in Table 6.

The results indicated that radionuclides are not predicted to reach groundwater (and by extension not predicted to reach the Columbia River) at levels above $4 \mathrm{mrem} / \mathrm{yr}$; therefore, the Columbia River protection RAGs have been attained.

\subsubsection{Nonradionuclides}

As described in Section 5.2.2, ethylene glycol and other COPCs either were not detected at the $116-\mathrm{KW}-4$ site or were detected at concentrations below background with the exception of hexavalent chromium, which was detected at a concentration below the soil RAG for river protection. The residual concentration of ethylene glycol detected at the $116-\mathrm{KE}-5$ site satisfies the $6,400 \mathrm{mg} / \mathrm{kg}$ soil RAG for river protection. Residual concentrations of lead and total chromium exceed their respective soil RAGs for river protection but, as described in Section 5.2.2, these constituents are not predicted to reach groundwater (and thus the Columbia River) within a 1,000-year time frame. Residual concentrations of all other metals for the 116-KE-5 site are below background levels or soil RAGs for river protection.

No nonradionuclide COCs were identified for the 100-K-55:1 or 100-K-56:1 pipelines, but hexavalent chromium concentrations were evaluated at discrete locations at the points of discharge from the $116-\mathrm{KW}-3$ and $116-\mathrm{KE}-4$ retention basins. As shown in Table 4, hexavalent chromium was not detected in the samples collected at the former discharge points from the $116-\mathrm{KW}-3$ retention basins. Concentrations of hexavalent chromium detected at the former discharge points of the 116-KW-4 retention basins are below the soil RAG for protection of the Columbia River and consistent with the residual concentrations for the entire basin footprint (BHI 2005d). 


\subsection{WAC 173-340 THREE-PART TEST FOR NONRADIONUCLIDES}

The WAC 173-340-740(7)(e) three-part test is required for nonradionuclide statistical verification data sets. No nonradionuclide COCs were identified for the 100-K-55:1 and 100-K-56:1 sites, and evaluation of RAG attainment for the 116-KW-4 and 116-KE-5 sites was based on discrete rather than statistical sample results.

The WAC 173-340-740(7)(e) three-part test is therefore not applicable to any of these data sets.

\subsection{RADIONUCLIDE RISK INFORMATION}

The radionuclide RAG for direct exposure is derived from the ROD (EPA 1997) and is expressed in terms of an allowable radiation dose rate above background (i.e., $15 \mathrm{mrem} / \mathrm{yr}$ ). The RAG evaluation (Section 5.0) involved using the RESRAD model to estimate total annual radiation dose rates for 1,000 years for comparison to the RAG. Radiation presents a carcinogenic risk, and the RESRAD model also calculates the excess lifetime cancer risk associated with the estimated radiation dose rates using the EPA's Health Effects Assessment Summary Tables (update dated April 16, 2001, "Update of Radionuclide Carcinogenicity Slope Factors," available on the Internet at www.epa.gov/radiation/heast). The "National Oil and Hazardous Substances Pollution Contingency Plan" (40 Code of Federal Regulations 300 ) presents a target range for residual risk of $10^{-4}$ to $10^{-6}$.

Figures 14, 15, and 16 illustrate excess lifetime cancer risk for the shallow zone as estimated using the RESRAD model for the 100-K-55:1 site, the 100-K-56:1 site, and the combined overburden for both sites, respectively. Because of radioactive decay, the risk decreases over time. The estimated risks for the 100-K-55:1 and 100-K-56:1 sites and their combined overburden is largest at present (year 2005), with estimates of $5.45 \times 10^{-5}, 1.20 \times 10^{-4}$, and $1.83 \times 10^{-5}$, respectively. These values decrease to $1.56 \times 10^{-15}, 6.03 \times 10^{-15}$, and $3.58 \times 10^{-21}$, respectively, in 1,000 years. The estimated risk values in 2018 are $2.97 \times 10^{-5}$ for the $100-\mathrm{K}-55: 1$ site, $6.66 \times 10^{-5}$ for the $100-\mathrm{K}-56: 1$ site, and $8.97 \times 10^{-6}$ for the combined overburden material. The 2018 date corresponds to the original 30-year site cleanup schedule of the Hanford Federal Facility Agreement and Consent Order (Ecology et al. 1989). 
Figure 14. Combined Radionuclide Excess Lifetime Cancer Risk for the 100-K-55:1 Shallow and Deep Zone Decision Units.

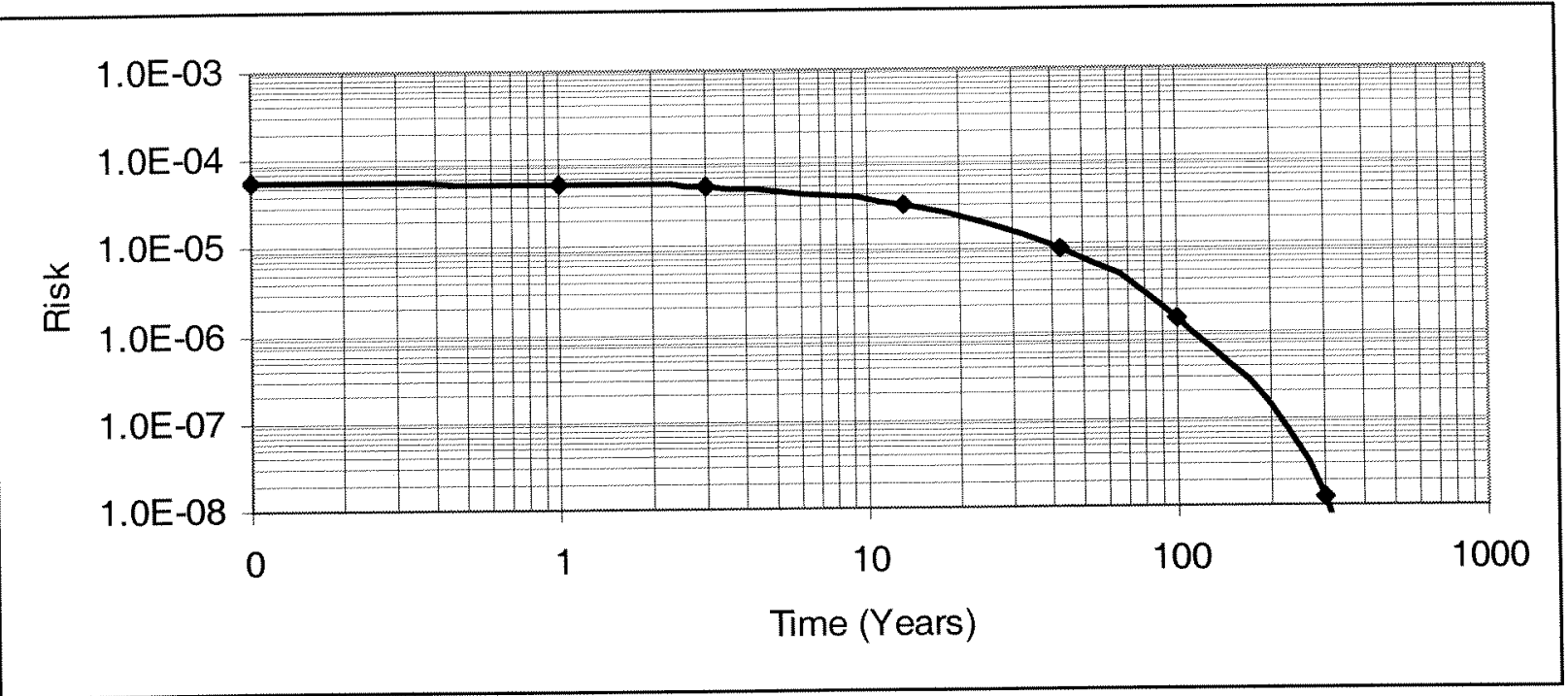

Figure 15. Combined Radionuclide Excess Lifetime Cancer Risk for the 100-K-56:1 Shallow and Deep Zone Decision Units.

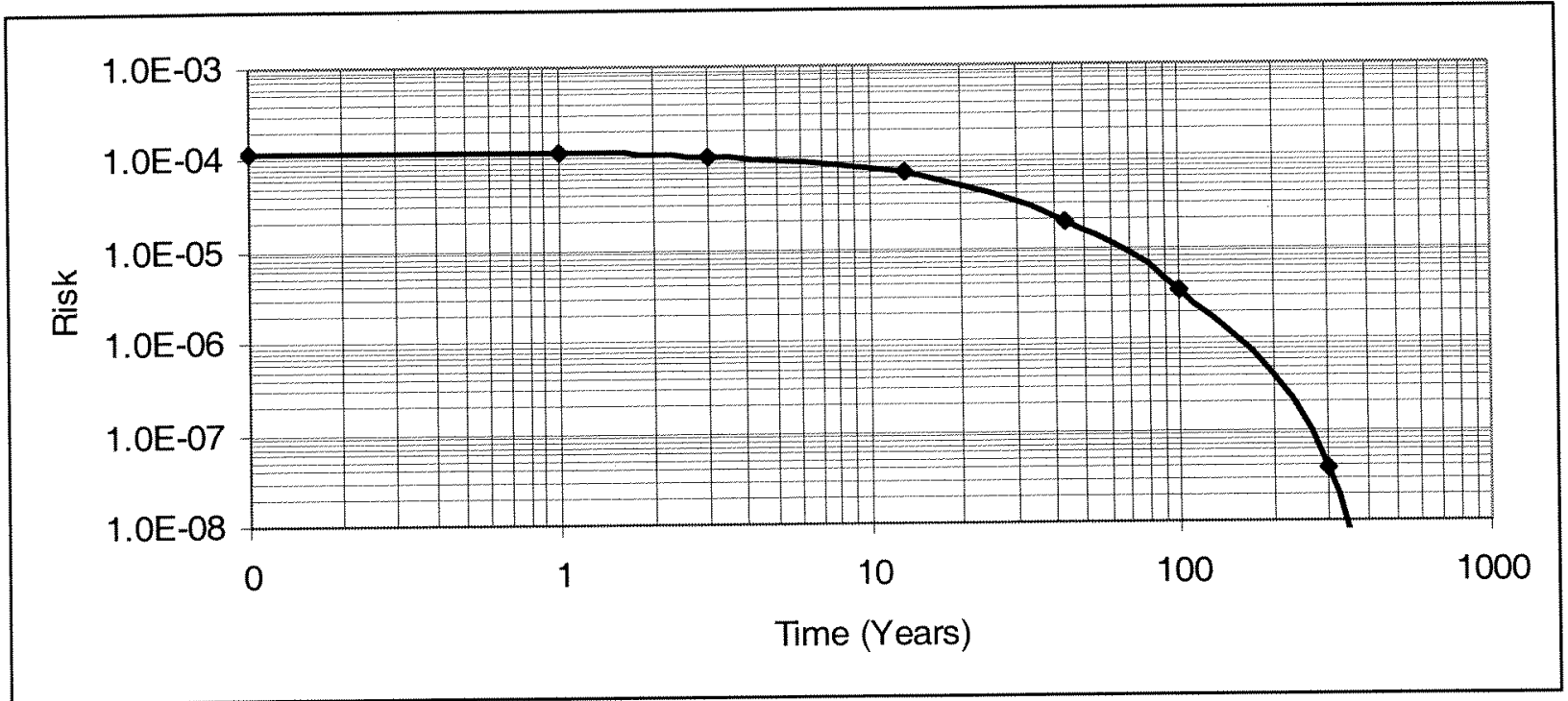


Figure 16. Radionuclide Excess Lifetime Cancer Risk for the 100-K-55:1 and 100-K-56:1 Sites Overburden Material.

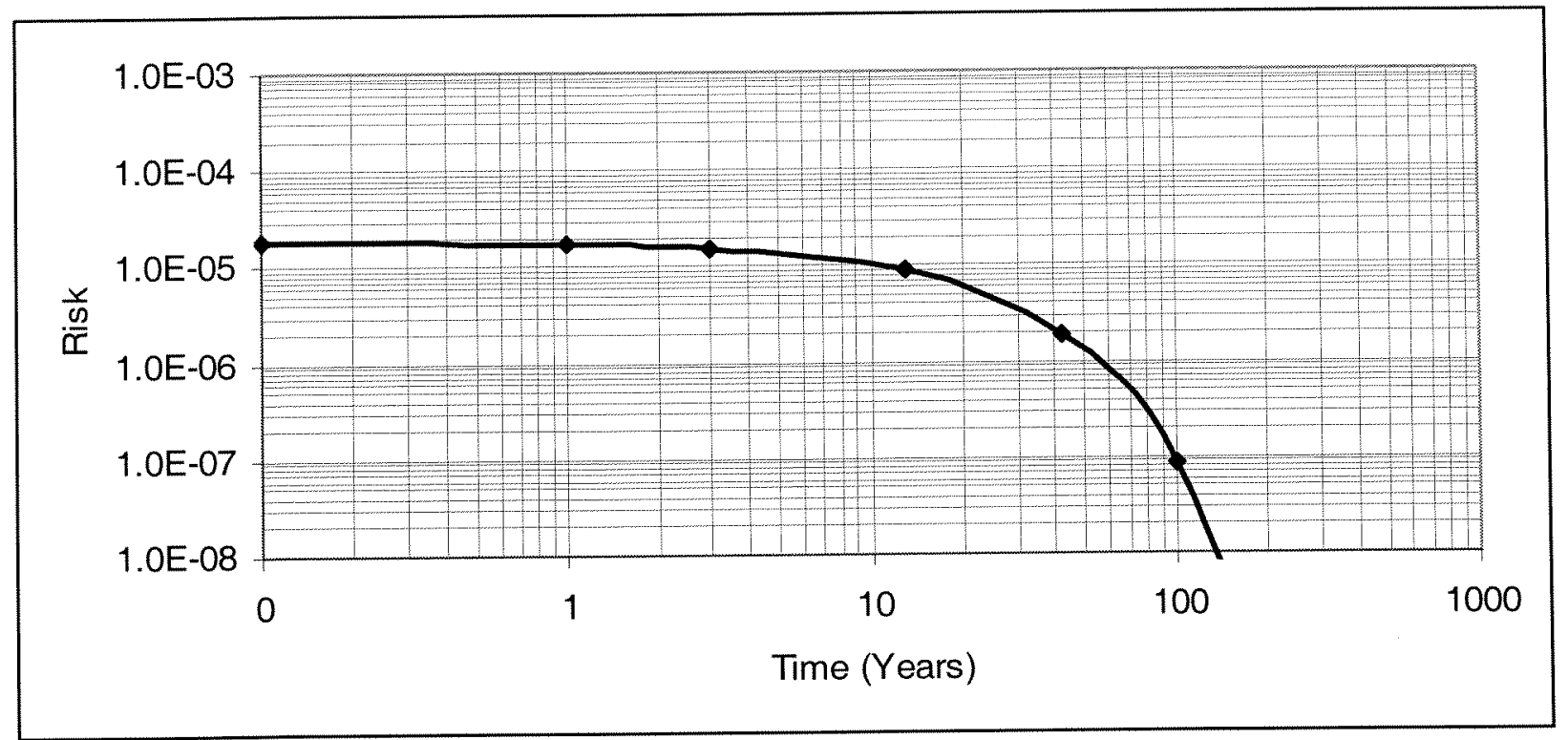

\subsection{STATEMENT OF PROTECTIVENESS}

This CVP demonstrates that remedial actions at the 100-K-55:1, 100-K-56:1, 116-KW-4, and 116-KE-5 sites have achieved the RAOs and corresponding RAGs established in the ROD (EPA 1997) and the RDR/RAWP (DOE-RL 2005b). The contaminated materials from these sites have been excavated and disposed at the ERDF. The remaining soils at the sites have been sampled, analyzed, and modeled, and the results do not preclude any future uses (as bounded by the rural-residential scenario), allow unrestricted use of shallow zone soils, and pose no threat to groundwater or the Columbia River. Institutional controls are required for the 100-K-55:1 and $100-K-56: 1$ sites to prevent drilling or excavation into deep zone soils. The 100-K-55:1, $100-\mathrm{K}-56: 1,116-\mathrm{KW}-4$, and $116-\mathrm{KE}-5$ sites are verified to be remediated in accordance with the ROD (EPA 1997) and may be backfilled. 


\subsection{REFERENCES}

40 CFR 300, "National Oil and Hazardous Substances Pollution Contingency Plan," Code of Federal Regulations, as amended.

65 FR 76708, "National Primary Drinking Water Regulations; Radionuclides; Final Rule," Federal Register, Vol. 65, No. 236, p. 76708, December 7, 2000.

ANL, 2004, RESRAD for Windows, Version 6.22, Argonne National Laboratory, Environmental Assessment Division, Argonne, Illinois.

BHI, 2000, Calculation of Hexavalent Chromium Carcinogenic Risk, 0100X-CA-V0031, Rev. 0, Bechtel Hanford, Inc., Richland, Washington.

$\mathrm{BHI}, 2001$, Calculation of Total Uranium Activity Corresponding to a Maximum Contaminant Level for Total Uranium of 30 Micrograms per Liter in Groundwater, 0100X-CA-V0038, Rev. 0, Bechtel Hanford, Inc., Richland, Washington.

$\mathrm{BHI}, 2004$, Cleanup Verification Package for 116-KW-3 Retention Basin, CVP-2004-00001, Rev. 0, Bechtel Hanford, Inc., Richland, Washington.

BHI, 2005a, 100 Area Analogous Sites RESRAD Calculations, 0100X-CA-V0050, Rev. 0, Bechtel Hanford, Inc., Richland, Washington.

BHI, 2005b, 100K Remedial Sampling Logbook, EL-1572-2, Bechtel Hanford, Inc., Richland, Washington.

BHI, 2005c, 100K Remedial Sampling Logbook, EL-1572-3, Bechtel Hanford, Inc., Richland, Washington.

$\mathrm{BHI}, 2005 \mathrm{~d}$, Cleanup Verification Package for the 116-KE-4 Retention Basin, CVP-2005-00002, Rev. 0, Bechtel Hanford, Inc., Richland, Washington.

DOE Order 5400.5, Radiation Protection of the Public and the Environment, U.S. Department of Energy, Washington, D.C.

DOE-RL, 1996, Hanford Site Background: Part 2, Soil Background for Radionuclides, DOE/RL-96-12, Rev. 0, U.S. Department of Energy, Richland Operations Office, Richland, Washington.

DOE-RL, 1998, Tri-Party Agreement Handbook Management Procedures, RL-TPA-90-0001, Guideline Number TPA-MP-14, "Maintenance of the Waste Information Data System (WIDS)," U.S. Department of Energy, Richland Operations Office, Richland, Washington. 
DOE-RL, 2001a, 100 Area Remedial Action Sampling and Analysis Plan, DOE/RL-96-22, Rev. 3, U.S. Department of Energy, Richland Operations Office, Richland, Washington.

DOE-RL, 2001b, Hanford Site Background: Part 1, Soil Background for Nonradioactive Analytes, DOE/RL-92-24, Rev. 4, U.S. Department of Energy, Richland Operations Office, Richland, Washington.

DOE-RL, 2005a, 100 Area Remedial Action Sampling and Analysis Plan, DOE/RL-96-22, Rev. 4, U.S. Department of Energy, Richland Operations Office, Richland, Washington.

DOE-RL, 2005b, Remedial Design Report/Remedial Action Work Plan for the 100 Area, DOE/RL-96-17, Rev. 5, U.S. Department of Energy, Richland Operations Office, Richland, Washington.

Ecology, EPA, and DOE, 1989, Hanford Federal Facility Agreement and Consent Order, 2 vols., as amended, Washington State Department of Ecology, U.S. Environmental Protection Agency, and U.S. Department of Energy, Olympia, Washington.

Ecology, 1994, Natural Background Soil Metals Concentrations in Washington State, Publication No. 94-115, Washington State Department of Ecology, Olympia, Washington.

EPA, 1994, Guidance Manual for the Integrated Exposure Uptake Biokinetic Model for Lead in Children, EPA/540/R-93/081, Publication No. 9285.7, U.S. Environmental Protection Agency, Washington D.C.

EPA, 1997, Amendment to the Interim Action Record of Decision for the 100-BC-1, 100-DR-1, and 100-HR-1 Operable Units, Hanford Site, Benton County, Washington, EPA/AMD/R10-97/044, U.S. Environmental Protection Agency, Region 10, Seattle, Washington.

EPA, Ecology, and RL, 1998, Environmental Restoration Contractor Meeting MinutesRemedial Action and Waste Disposal Unit Manager's Meeting - 100 Area, Draft, dated July 6, 1998, U.S. Environmental Protection Agency, Washington State Department of Ecology, and U.S. Department of Energy, Richland Operations Office, Richland, Washington.

WAC 173-340, 1996, "Model Toxics Control Act - Cleanup," Washington Administrative Code. 
CVP-2005-00006

Rev. 0 


\section{APPENDIX A}

SUMMARY OF VERIFICATION SOIL SAMPLING AND ANALYTICAL RESULTS 
CVP-2005-00006

Rev. 0 
Table A-1. 100-K-55:1 Deep Zone Cleanup Verification Data.

\begin{tabular}{|c|c|c|c|c|c|c|c|c|c|c|c|}
\hline \multirow{2}{*}{$\begin{array}{c}\text { Sampling } \\
\text { Area }\end{array}$} & \multirow{2}{*}{$\begin{array}{l}\text { HEIS } \\
\text { Number }\end{array}$} & \multirow{2}{*}{$\begin{array}{c}\text { Sample } \\
\text { Date }\end{array}$} & \multicolumn{3}{|c|}{ Cesium-137 } & \multicolumn{3}{|c|}{ Europium-152 } & \multicolumn{3}{|c|}{ Europium-154 } \\
\hline & & & $\mathrm{pCi} / \mathrm{g}$ & $\mathbf{Q}$ & MDA & $\mathrm{pCi} / \mathrm{g}$ & $\mathbf{Q}$ & MDA & $\mathrm{pCi} / \mathrm{g}$ & $\mathbf{Q}$ & MDA \\
\hline $\mathrm{A} 1$ & $\mathrm{~J} 02 \mathrm{H} 20$ & $02 / 15 / 05$ & $2.88 E+00$ & & $5.7 E=02$ & 1.9E-01 & U & $1.9 \mathrm{E}-01$ & $1.7 E-01$ & $U$ & 1.7E-01 \\
\hline $\begin{array}{c}\text { Duplicate of } \\
\text { J02H2O }\end{array}$ & J02HW9 & $02 / 15 / 05$ & $3.85 E+00$ & & 4.4E-02 & 2.05E-01 & & $1.1 E-01$ & 1.1E-01 & $\mathrm{U}$ & $1.1 \mathrm{E}-01$ \\
\hline $\mathrm{D} 2$ & $\mathrm{~J} 02 \mathrm{H} 14$ & $01 / 26 / 05$ & 4.1E-02 & U & 4.1E-02 & $1.4 \mathrm{E}-01$ & $\mathrm{U}$ & $1.4 \mathrm{E}-01$ & $1.5 \mathrm{E}-01$ & U & 1.5E-01 \\
\hline $\begin{array}{c}\text { Duplicate of } \\
\text { J02H14 }\end{array}$ & $\mathrm{J} 02 \mathrm{H} 15$ & $01 / 26 / 05$ & $3.5 \mathrm{E}-02$ & $u$ & $3.5 E-02$ & $9.7 E-02$ & $\mathrm{U}$ & 9.7E-02 & 1.3E-01 & $U$ & 1.3E-01 \\
\hline $\mathrm{A} 2$ & $\mathrm{~J} 02 \mathrm{H} 19$ & $02 / 15 / 05$ & 4.5E-02 & U & 4.5E-02 & $6.39 \mathrm{E}-01$ & & 1.0E-01 & $1.7 E-01$ & U & 1.7E-01 \\
\hline A3 & $\mathrm{J} 02 \mathrm{H} 18$ & $02 / 15 / 05$ & $8.2 E-02$ & & $5.4 \mathrm{E}-02$ & $1.16 \mathrm{E}+00$ & & $9.7 \mathrm{E}-02$ & 2.3E-01 & $\mathrm{U}$ & 2.3E-01 \\
\hline B4 & $\mathrm{J} 02 \mathrm{H} 17$ & $02 / 15 / 05$ & 1.49E-01 & & $5.5 \mathrm{E}-02$ & $1.00 \mathrm{E}+00$ & & $9.9 \mathrm{E}-02$ & 2.9E-01 & U & 2.9E-01 \\
\hline B5 & $\mathrm{J} 02 \mathrm{H} 07$ & $01 / 26 / 05$ & $3.0 \mathrm{E}-02$ & $\mathrm{U}$ & 3.0E-02 & $7.4 \mathrm{E}-02$ & $U$ & 7.4E-02 & 8.5E-02 & $\mathrm{U}$ & 8.5E-02 \\
\hline $\mathrm{B} 6$ & $\mathrm{~J} 02 \mathrm{H} 08$ & $01 / 26 / 05$ & $3.6 \mathrm{E}-02$ & $\mathrm{U}$ & 3.6E-02 & $8.8 \mathrm{E}-02$ & U & $8.8 \mathrm{E}-02$ & $1.1 \mathrm{E}-01$ & $U$ & 1.1E-01 \\
\hline $\mathrm{C} 7$ & $\mathrm{~J} 02 \mathrm{H} 09$ & $01 / 26 / 05$ & $6.5 \mathrm{E}-02$ & & $3.4 \mathrm{E}-02$ & $3.03 \mathrm{E}-01$ & & 7.9E-02 & $1.2 \mathrm{E}-01$ & $U$ & 1.2E-01 \\
\hline $\mathrm{C} 8$ & $\mathrm{~J} 02 \mathrm{H} 10$ & $01 / 26 / 05$ & 3.1E-02 & $U$ & $3.1 \mathrm{E}-02$ & 7.8E-02 & U & $7.8 \mathrm{E}-02$ & 9.9E-02 & U & $9.9 \mathrm{E}-02$ \\
\hline $\mathrm{C} 9$ & $\mathrm{~J} 02 \mathrm{H} 11$ & $01 / 26 / 05$ & $5.90 \mathrm{E}-01$ & & $2.4 \mathrm{E}-02$ & $1.85 E+00$ & & $4.2 \mathrm{E}-02$ & $2.88 \mathrm{E}-01$ & & $5.8 \mathrm{E}-02$ \\
\hline D10 & $\mathrm{J} 02 \mathrm{H} 12$ & $01 / 26 / 05$ & $3.8 \mathrm{E}-02$ & U & 3.8E-02 & $1.0 \mathrm{E}-01$ & U & 1.0E-01 & 1.4E-01 & $\mathrm{U}$ & $1.4 \mathrm{E}-01$ \\
\hline D1 & $\mathrm{J} 02 \mathrm{H} 13$ & $01 / 26 / 05$ & 8.9E-02 & & 1.0E-01 & 1.24E-01 & & $3.5 \mathrm{E}-02$ & $1.1 \mathrm{E}-01$ & $u$ & $1.1 \mathrm{E}-01$ \\
\hline
\end{tabular}

NOTE: The following acronyms and abbreviations apply to all tables in this appendix.

HEIS = Hanford Environmental Information system

MDA = minimum detectable activity

$\mathrm{PQL}=$ practical quantitation limit

$\mathrm{Q} \quad$ = qualifier

$\mathrm{U}=$ undetected 
CVP-2005-00006

Rev. 0

Table A-2. 100-K-55:1 Shallow Zone Cleanup Verification Data.

\begin{tabular}{|c|c|c|c|c|c|c|c|c|c|c|c|}
\hline \multirow{2}{*}{$\begin{array}{l}\text { Sampling } \\
\text { Area }\end{array}$} & \multirow{2}{*}{$\begin{array}{l}\text { HEIS } \\
\text { Number }\end{array}$} & \multirow{2}{*}{$\begin{array}{c}\text { Sample } \\
\text { Date }\end{array}$} & \multicolumn{3}{|c|}{ Cesium-137 } & \multicolumn{3}{|c|}{ Europium-152 } & \multicolumn{3}{|c|}{ Europium-154 } \\
\hline & & & $\mathrm{pCi} / \mathrm{g}$ & $\mathbf{Q}$ & MDA & $\mathrm{pCi} / \mathrm{g}$ & $Q$ & MDA & $\mathrm{pCi} / \mathrm{g}$ & $\mathbf{Q}$ & MDA \\
\hline A4 & J02HK9 & $02 / 23 / 05$ & 3.51E-01 & & $5.5 E-02$ & $1.26 \mathrm{E}+00$ & & $1.0 \mathrm{E}-01$ & $2.48 \mathrm{E}-01$ & & 1.6E-01 \\
\hline $\begin{array}{c}\text { Duplicate of } \\
\text { J02HK9 }\end{array}$ & J02HLO & $02 / 23 / 05$ & 5.39E-01 & & 5.3E-02 & $2.04 E+00$ & & 1.1E-01 & $1.7 E-01$ & $U$ & 1.7E-01 \\
\hline F4 & J02HNO & $2 / 28 / 05$ & 3.0E-02 & U & $3.0 \mathrm{E}-02$ & 7.0E-02 & U & 7.0E-02 & $1.1 E-01$ & $U$ & $1.1 \mathrm{E}-01$ \\
\hline $\begin{array}{c}\text { Duplicate of } \\
\text { J02HNO }\end{array}$ & J02HN1 & $02 / 28 / 05$ & $3.4 \mathrm{E}-02$ & u & $3.4 \mathrm{E}-02$ & $1.2 \mathrm{E}-01$ & $u$ & 1.2E-01 & 1.3E-01 & U & 1.3E-01 \\
\hline $\mathrm{A} 1$ & J02HK6 & 02/23/05 & $2.9 \mathrm{E}-02$ & $\mathrm{U}$ & $3.4 \mathrm{E}-02$ & 8.1E-02 & & 7.3E-02 & $9.5 \mathrm{E}-02$ & $\mathrm{U}$ & 9.5E-02 \\
\hline $\mathrm{A} 2$ & J02HK7 & $02 / 23 / 05$ & $4.1 \mathrm{E}-02$ & $U$ & $4.1 \mathrm{E}-02$ & 1.4E-01 & & $1.4 \mathrm{E}-01$ & 1.5E-01 & $\mathrm{U}$ & 1.5E-01 \\
\hline $\mathrm{A} 3$ & J02HK8 & $02 / 23 / 05$ & $1.42 E-01$ & & $3.6 \mathrm{E}-02$ & 4.62E-01 & & $7.8 \mathrm{E}-02$ & 1.1E-01 & $U$ & 1.1E-01 \\
\hline B5 & $\mathrm{J} 02 \mathrm{HL} 1$ & $02 / 23 / 05$ & $6.3 E-02$ & & 3.6E-02 & 1.82E-01 & & $7.4 \mathrm{E}-02$ & $1.3 \mathrm{E}-01$ & $U$ & 1.3E-01 \\
\hline B6 & J02HL2 & $02 / 23 / 05$ & $3.2 \mathrm{E}-02$ & $U$ & $3.2 E-02$ & 1.16E-01 & & 7.5E-02 & 1.0E-01 & U & $1.0 \mathrm{E}-01$ \\
\hline B7 & J02HL3 & $02 / 23 / 05$ & 4.0E-02 & $U$ & $4.0 \mathrm{E}-02$ & 1.3E-01 & & 1.3E-01 & 1.6E-01 & U & 1.6E-01 \\
\hline$B 8$ & $\mathrm{~J} 02 \mathrm{HL} 4$ & $02 / 23 / 05$ & 2.26E-01 & & 4.7E-02 & $6.4 \mathrm{E}-01$ & & $7.8 \mathrm{E}-02$ & 1.3E-01 & $U$ & $1.3 \mathrm{E}-01$ \\
\hline $\mathrm{Cg}$ & J02HL5 & $02 / 23 / 05$ & $3.4 \mathrm{E}-02$ & $\mathrm{U}$ & $3.4 \mathrm{E}-02$ & 6.6E-02 & & 7.5E-02 & 1.0E-01 & $\mathrm{U}$ & 1.0E-01 \\
\hline C10 & J02HL6 & $02 / 23 / 05$ & $2.90 E-01$ & & $5.5 \mathrm{E}-02$ & 3.09E-01 & & $1.0 \mathrm{E}-01$ & 1.6E-01 & $\mathrm{U}$ & 1.6E-01 \\
\hline $\mathrm{C} 1$ & $\mathrm{~J} 02 \mathrm{HL} 7$ & $02 / 23 / 05$ & $3.20 \mathrm{E}-01$ & & 4.3E-02 & 7.07E-01 & & 8.5E-02 & 1.3E-01 & $U$ & 1.3E-01 \\
\hline $\mathrm{C} 2$ & J02HL8 & $02 / 23 / 05$ & 2.34E-01 & & 4.0E-02 & $2.79 \mathrm{E}-01$ & & 8.4E-02 & $1.1 \mathrm{E}-01$ & $U$ & 1.1E-01 \\
\hline D3 & J02HL9 & $02 / 24 / 05$ & $4.4 \mathrm{E}-02$ & & $3.5 \mathrm{E}-02$ & $1.4 \mathrm{E}-01$ & & $9.2 E-02$ & $1.4 \mathrm{E}-01$ & $U$ & 1.4E-01 \\
\hline D4 & J02HMO & $02 / 24 / 05$ & 3.7E-02 & & $2.8 \mathrm{E}-02$ & 1.61E-01 & & 8.2E-02 & 1.3E-01 & $\mathrm{U}$ & 1.3E-01 \\
\hline D5 & $\mathrm{J} 02 \mathrm{HM} 1$ & $02 / 24 / 05$ & $6.1 \mathrm{E}-01$ & & $4.5 E-02$ & $2.28 \mathrm{E}-01$ & & 9.3E-02 & $1.6 \mathrm{E}-01$ & $U$ & 1.6E-01 \\
\hline D6 & $\mathrm{J} 02 \mathrm{HM} 2$ & $02 / 24 / 05$ & $3.98 E-01$ & & 7.7E-02 & $3.87 \mathrm{E}+00$ & & 1.3E-01 & 5.21E-01 & & 1.7E-01 \\
\hline E7 & J02HM3 & $02 / 24 / 05$ & 5.7E-01 & & 4.3E-02 & $3.15 \mathrm{E}-01$ & & 7.9E-02 & $1.1 \mathrm{E}-01$ & $\mathrm{U}$ & 1.1E-01 \\
\hline E8 & J02HM4 & $02 / 24 / 05$ & 2.05E-01 & & $3.8 \mathrm{E}-02$ & 1.4E-01 & $\mathrm{U}$ & 1.4E-01 & 1.6E-01 & $U$ & 1.6E-01 \\
\hline E9 & J02HM5 & $02 / 24 / 05$ & $8.44 \mathrm{E}-01$ & & $6.3 E-02$ & $1.07 E+00$ & & $1.2 \mathrm{E}-01$ & $1.9 \mathrm{E}-01$ & $U$ & $1.9 \mathrm{E}-01$ \\
\hline E10 & J02HM6 & $02 / 24 / 05$ & $6.3 \mathrm{E}-02$ & $U$ & $6.3 \mathrm{E}-02$ & $8.2 E-02$ & U & $8.2 E-02$ & $1.2 \mathrm{E}-01$ & U & 1.2E-01 \\
\hline $\mathrm{F} 1$ & J02HM7 & $02 / 24 / 05$ & 3.6E-02 & $U$ & 3.6E-02 & $1.2 \mathrm{E}-01$ & $\mathrm{U}$ & $1.2 \mathrm{E}-01$ & $1.4 \mathrm{E}-01$ & U & 1.4E-01 \\
\hline F2 & J02HM8 & $02 / 24 / 05$ & $3.1 \mathrm{E}-02$ & U & $3.1 \mathrm{E}-02$ & 7.5E-02 & U & 7.5E-02 & 1.1E-01 & U & 1.1E-01 \\
\hline F3 & J02HM9 & $02 / 24 / 05$ & 3.3E-02 & U & 3.3E-02 & $7.8 \mathrm{E}-02$ & $\mathrm{U}$ & $7.8 \mathrm{E}-02$ & 1.3E-01 & U & 1.3E-01 \\
\hline
\end{tabular}


Table A-3. 100-K-56:1 Deep Zone Cleanup Verification Data.

\begin{tabular}{|c|c|c|c|c|c|c|c|c|c|c|c|}
\hline \multirow{2}{*}{$\begin{array}{c}\text { Sampling } \\
\text { Area }\end{array}$} & \multirow{2}{*}{$\begin{array}{c}\text { HEIS } \\
\text { Number }\end{array}$} & \multirow{2}{*}{$\begin{array}{c}\text { Sample } \\
\text { Date }\end{array}$} & \multicolumn{3}{|c|}{ Cesium-137 } & \multicolumn{3}{|c|}{ Europium-152 } & \multicolumn{3}{|c|}{ Europium-154 } \\
\hline & & & $\mathrm{pCi} / \mathrm{g}$ & Q & MDA & $\mathrm{pCi} / \mathrm{g}$ & $Q$ & MDA & $\mathrm{pCi} / \mathrm{g}$ & $\mathbf{Q}$ & MDA \\
\hline A1 & J037K9 & $6 / 15 / 05$ & $4.57 \mathrm{E}+00$ & & 1.0E-01 & $3.14 \mathrm{E}+01$ & & 3.5E-01 & $4.08 E+00$ & & $3.1 \mathrm{E}-01$ \\
\hline $\mathrm{A} 2$ & J037L0 & $6 / 15 / 05$ & $2.83 E+00$ & & 8.5E-02 & $1.02 E+01$ & & 1.5E-01 & $1.42 E+00$ & & 1.4E-01 \\
\hline $\mathrm{A} 3$ & J037L1 & $6 / 15 / 05$ & $2.16 \mathrm{E}+01$ & & $1.2 E-01$ & $8.83 E+00$ & & 3.7E-01 & $1.18 \mathrm{E}+00$ & & $2.3 \mathrm{E}-01$ \\
\hline B4 & J037L2 & $6 / 15 / 05$ & $5.40 \mathrm{E}+00$ & & $9.2 \mathrm{E}-02$ & $5.23 E+00$ & & 1.5E-01 & $5.68 \mathrm{E}-01$ & & 1.7E-01 \\
\hline$B 5$ & J037L3 & $6 / 15 / 05$ & $2.86 \mathrm{E}+01$ & & 1.1E-01 & $1.04 \mathrm{E}+01$ & & $2.4 \mathrm{E}-01$ & $1.78 \mathrm{E}+00$ & & 1.7E-01 \\
\hline B6 & J037L4 & $6 / 16 / 05$ & 8.49E-01 & & 3.6E-02 & $5.06 \mathrm{E}-01$ & & 9.4E-02 & 1.4E-01 & U & $1.4 \mathrm{E}-01$ \\
\hline $\mathrm{C7}$ & J037L5 & $6 / 16 / 05$ & 1.15E-01 & & $4.9 \mathrm{E}-02$ & $1.0 \mathrm{E}-01$ & $u$ & $1.0 \mathrm{E}-01$ & $1.3 \mathrm{E}-01$ & 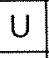 & 1.3E-01 \\
\hline $\mathrm{C} 8$ & J037L6 & $6 / 16 / 05$ & $3.28 \mathrm{E}+00$ & & 8.6E-02 & $8.48 E+00$ & & 1.6E-01 & $1.31 E+00$ & & $1.9 \mathrm{E}-01$ \\
\hline $\mathrm{Cg}$ & J037L7 & $6 / 16 / 05$ & $9.24 \mathrm{E}-01$ & & 5.1E-02 & $1.55 \mathrm{E}+00$ & & $1,2 \mathrm{E}-01$ & $1.8 \mathrm{E}-01$ & $U$ & $1.8 \mathrm{E}-01$ \\
\hline $\begin{array}{l}\text { Duplicate of } \\
\text { J037L7 }\end{array}$ & J037L8 & $6 / 16 / 05$ & $4.5 \mathrm{E}-02$ & $U$ & $4.5 \mathrm{E}-02$ & $1.2 E-01$ & $u$ & $1.2 \mathrm{E}-01$ & 1.6E-01 & $U$ & $1.6 \mathrm{E}-01$ \\
\hline
\end{tabular}

Table A-4. 100-K-56:1 Shallow Zone Cleanup Verification Data.

\begin{tabular}{|c|c|c|c|c|c|c|c|c|c|c|c|}
\hline \multirow{2}{*}{$\underset{\text { Area }}{\text { Sampling }}$} & \multirow{2}{*}{$\begin{array}{c}\text { HEIS } \\
\text { Number }\end{array}$} & \multirow{2}{*}{$\begin{array}{l}\text { Sample } \\
\text { Date }\end{array}$} & \multicolumn{3}{|c|}{ Cesium-137 } & \multicolumn{3}{|c|}{ Europium-152 } & \multicolumn{3}{|c|}{ Europium-154 } \\
\hline & & & $\mathrm{pCi} / \mathrm{g}$ & $\mathbf{Q}$ & MDA & $\mathrm{pCi} / \mathrm{g}$ & $\mathbf{Q}$ & MDA & $\mathrm{pCi} / \mathrm{g}$ & $\mathbf{Q}$ & MDA \\
\hline A1 & J03D45 & $6 / 23 / 05$ & 1.21E-01 & & 4.6E-02 & $3.42 \mathrm{E}-01$ & & 7.0E-02 & 1.3E-01 & $\mathrm{U}$ & 1.3E-01 \\
\hline $\begin{array}{c}\text { Duplicate of } \\
\text { J03D45 }\end{array}$ & J03D46 & $6 / 23 / 05$ & 1.17E-01 & & 4.8E-02 & $4.89 E-01$ & & $8.9 E-02$ & 1.6E-01 & $\mathrm{U}$ & 1.6E-01 \\
\hline $\mathrm{A} 2$ & J03D47 & $6 / 23 / 05$ & 1.44E-01 & & $4.0 \mathrm{E}-02$ & $1.41 E+00$ & & $1.2 \mathrm{E}-01$ & 2.2E-01 & $U$ & 2.2E-01 \\
\hline A3 & J03D48 & $6 / 23 / 05$ & $3.10 \mathrm{E}-01$ & & $5.0 \mathrm{E}-02$ & $4.10 \mathrm{E}-01$ & & $1.0 \mathrm{E}-01$ & 1.7E-01 & U & 1.7E-01 \\
\hline $\mathrm{A} 4$ & J03D49 & $6 / 23 / 05$ & 1.50E-01 & & 4.0E-02 & 1.5E-01 & $\mathrm{U}$ & 1.5E-01 & 1.1E-01 & U & $1.1 \mathrm{E}-01$ \\
\hline B5 & J03D50 & $6 / 23 / 05$ & $2.38 \mathrm{E}+00$ & & 7.2E-02 & $1.48 \mathrm{E}+00$ & & 1.3E-01 & 2.4E-01 & $\mathrm{U}$ & 2.4E-01 \\
\hline B6 & J03D51 & $6 / 23 / 05$ & $2.62 \mathrm{E}-01$ & & $3.5 \mathrm{E}-02$ & 1.3E-01 & U & 1.3E-01 & 1.0E-01 & $\mathrm{U}$ & 1.0E-01 \\
\hline$B 7$ & J03D52 & $6 / 23 / 05$ & $2.05 E+00$ & & $6.2 \mathrm{E}-02$ & $2.17 \mathrm{E}+00$ & & 1.1E-01 & 4.7E-01 & $U$ & 4.7E-01 \\
\hline B8 & J03D53 & $6 / 23 / 05$ & $3.9 \mathrm{E}-02$ & $\mathrm{U}$ & 3.9E-02 & $9.4 \mathrm{E}-02$ & $\mathrm{U}$ & $9.4 \mathrm{E}-02$ & 1.3E-01 & $U$ & $1.3 \mathrm{E}-01$ \\
\hline $\mathrm{C9}$ & J03D54 & $6 / 23 / 05$ & $2.8 \mathrm{E}-02$ & $U$ & $2.8 \mathrm{E}-02$ & 1.1E-01 & U & 1.1E-01 & 1.0E-01 & $U$ & $1.0 \mathrm{E}-01$ \\
\hline $\mathrm{C} 10$ & J03D55 & $6 / 23 / 05$ & $3.98 \mathrm{E}-01$ & & $3.7 \mathrm{E}-02$ & 7.7E-02 & $U$ & 7.7E-02 & 1.0E-01 & U & 1.0E-01 \\
\hline $\mathrm{C} 1$ & J03D56 & $6 / 23 / 05$ & 1.96E-01 & & 5.3E-02 & $1.1 \mathrm{E}-01$ & $U$ & $1.1 \mathrm{E}-01$ & $1.5 \mathrm{E}-01$ & $U$ & 1.5E-01 \\
\hline $\mathrm{C} 2$ & J03D57 & $6 / 23 / 05$ & 9.3E-02 & & $3.4 \mathrm{E}-02$ & $1.1 \mathrm{E}-01$ & $U$ & 1.1E-01 & 1.0E-01 & $\mathrm{U}$ & 1.0E-01 \\
\hline D3 & J03D58 & $6 / 23 / 05$ & 3.0E-02 & $\mathrm{U}$ & $3.0 \mathrm{E}-02$ & 7.0E-02 & $\mathrm{U}$ & $7.0 \mathrm{E}-02$ & 1.0E-01 & $\mathrm{U}$ & $1.0 \mathrm{E}-01$ \\
\hline $\mathrm{D} 4$ & J03D59 & $6 / 23 / 05$ & 3.3E-02 & $U$ & 3.3E-02 & 1.0E-01 & $\mathrm{U}$ & 1.0E-01 & $1.2 \mathrm{E}-01$ & U & 1.2E-01 \\
\hline D5 & J03D60 & $6 / 23 / 05$ & 4.0E-02 & $U$ & 4.0E-02 & 1.1E-01 & $\mathrm{U}$ & 1.1E-01 & $1.3 \mathrm{E}-01$ & $U$ & 1.3E-01 \\
\hline D6 & J03D61 & $6 / 23 / 05$ & $4.42 \mathrm{E}-01$ & & 4.3E-02 & $1.6 \mathrm{E}-01$ & $U$ & 1.6E-01 & $1.1 \mathrm{E}-01$ & $U$ & $1.1 \mathrm{E}-01$ \\
\hline E7 & J03D62 & $6 / 23 / 05$ & 3.81E-01 & & 8.7E-02 & $1.04 \mathrm{E}+01$ & & 1.6E-01 & $1.1 E+00$ & $U$ & $1.1 \mathrm{E}+00$ \\
\hline E8 & J03D63 & $6 / 23 / 05$ & 7.3E-02 & & 3.9E-02 & $3.42 \mathrm{E}-01$ & & 9.9E-02 & 1.6E-01 & U & 1.6E-01 \\
\hline E9 & J03D64 & $6 / 23 / 05$ & $2.86 \mathrm{E}-01$ & & $4.8 \mathrm{E}-02$ & $2.40 \mathrm{E}-01$ & & 1.0E-01 & $1.4 \mathrm{E}-01$ & $U$ & 1.4E-01 \\
\hline E10 & J03D65 & $6 / 23 / 05$ & 9.3E-02 & & $3.6 \mathrm{E}-02$ & $1.2 \mathrm{E}-01$ & $\mathrm{U}$ & $1.2 \mathrm{E}-01$ & 9.6E-02 & $U$ & $9.6 \mathrm{E}-02$ \\
\hline
\end{tabular}


Table A-5. Overburden Cleanup Verification Data. (2 Pages)

\begin{tabular}{|c|c|c|c|c|c|c|c|c|c|c|c|}
\hline \multirow{2}{*}{$\begin{array}{l}\text { Sampling } \\
\text { Area }\end{array}$} & \multirow{2}{*}{$\begin{array}{l}\text { HEIS } \\
\text { Number }\end{array}$} & \multirow{2}{*}{$\begin{array}{l}\text { Sample } \\
\text { Date }\end{array}$} & \multicolumn{3}{|c|}{ Cesium-137 } & \multicolumn{3}{|c|}{ Europium-152 } & \multicolumn{3}{|c|}{ Europium-154 } \\
\hline & & & $\mathrm{pCi} / \mathrm{g}$ & $Q$ & MDA & $\mathrm{pCl} / \mathrm{g}$ & $\mathbf{Q}$ & MDA & $\mathrm{pCi} / \mathrm{g}$ & $\mathbf{Q}$ & MDA \\
\hline A4 & J02HN7 & $02 / 18 / 05$ & $1.61 E+00$ & & $5.4 \mathrm{E}-02$ & $2.65 E=01$ & & $8.5 E-02$ & $1.2 \mathrm{E}-01$ & U & 1.2E-01 \\
\hline $\begin{array}{c}\text { Duplicate of } \\
\text { J02HN7 }\end{array}$ & J02HN8 & $02 / 18 / 05$ & $1.80 E+00$ & & $3.9 \mathrm{E}-02$ & 2.03E-01 & & 8.2E-02 & $1.2 \mathrm{E}-01$ & U & 1.2E-01 \\
\hline $\mathrm{F} 4$ & J02HR8 & $3 / 01 / 05$ & $1.48 E-01$ & & $4.0 \mathrm{E}-02$ & $.14 \mathrm{E}-01$ & & 8.3E-02 & 1.3E-01 & U & 1.3E-01 \\
\hline $\begin{array}{c}\text { Duplicate of } \\
\text { J02HR8 }\end{array}$ & J02HR9 & 03/01/05 & 2.00E-01 & & 4.0E-02 & $1.2 \mathrm{E}-01$ & U & $1.2 \mathrm{E}-01$ & 1.2E-01 & $\mathrm{U}$ & 1.2E-01 \\
\hline $\mathrm{K} 4$ & J02HV9 & $02 / 17 / 05$ & 3.9E-02 & u & $3.9 \mathrm{E}-02$ & $9.5 \mathrm{E}-02$ & u & $9.5 E-02$ & $1.4 \mathrm{E}-01$ & U & 1.4E-01 \\
\hline $\begin{array}{c}\text { Duplicate of } \\
\mathrm{J} 02 \mathrm{HV} 9\end{array}$ & J02HWO & $02 / 17 / 05$ & $4.5 \mathrm{E}-02$ & U & 4.5E-02 & 1.4E-01 & u & $1.4 \mathrm{E}-01$ & 1.7E-01 & $U$ & 1.7E-01 \\
\hline A1 & J02HN4 & $02 / 18 / 05$ & $2.94 \mathrm{E}-01$ & & 4.1E-02 & $1.59 \mathrm{E}-01$ & & 7.5E-02 & 1.3E-01 & U & 1.3E-01 \\
\hline $\mathrm{A} 2$ & J02HN5 & $02 / 18 / 05$ & $1.94 \mathrm{E}-01$ & & $4.4 \mathrm{E}-02$ & $1.44 \mathrm{E}+00$ & & $8.4 \mathrm{E}-02$ & $3.27 \mathrm{E}-01$ & & 1.4E-01 \\
\hline A3 & J02HN6 & $02 / 18 / 05$ & $3.95 E-01$ & & $4.3 \mathrm{E}-02$ & $1.5 E-01$ & $u$ & $1.5 \mathrm{E}-01$ & $1.5 E-01$ & U & 1.5E-01 \\
\hline B5 & J02HN9 & $03 / 03 / 05$ & $3.44 \mathrm{E}-01$ & & $4.4 \mathrm{E}-02$ & 2.03E-01 & & $8.4 \mathrm{E}-02$ & 1.2E-01 & $\mathrm{U}$ & 1.2E-01 \\
\hline B6 & J02HPO & $03 / 03 / 05$ & $8.1 E-02$ & & 3.7E-02 & $1.61 E-01$ & & $8.9 \mathrm{E}-02$ & 1.5E-01 & $U$ & $1.5 \mathrm{E}-01$ \\
\hline B7 & J02HP1 & $03 / 03 / 05$ & $5.60 \mathrm{E}-01$ & & $5.3 \mathrm{E}-02$ & $1.12 E+00$ & & $8.9 \mathrm{E}-02$ & $1.6 \mathrm{E}-01$ & U & 1.6E-01 \\
\hline$B 8$ & J02HP2 & $03 / 03 / 05$ & 2.19E-01 & & 3.9E-02 & $1.2 \mathrm{E}-01$ & U & $1.2 \mathrm{E}-01$ & $1.4 \mathrm{E}-01$ & U & 1.4E-01 \\
\hline $\mathrm{C9}$ & J02HP3 & $02 / 22 / 05$ & 4.4E-02 & & 3.0E-02 & $1.2 \mathrm{E}-01$ & $U$ & 1.2E-01 & $1.2 E-01$ & $\mathrm{u}$ & 1.2E-01 \\
\hline $\mathrm{C} 10$ & J02HP4 & $02 / 22 / 05$ & 5.3E-02 & & $3.5 \mathrm{E}-02$ & 2.17E-01 & & $7.2 \mathrm{E}-02$ & 1.1E-01 & $\mathrm{U}$ & 1.1E-01 \\
\hline $\mathrm{C} 1$ & J02HP5 & $02 / 22 / 05$ & $8.2 E-02$ & & $3.6 \mathrm{E}-02$ & $1.21 E-01$ & & 7.7E-02 & 1.3E-01 & $\mathrm{u}$ & 1.3E-01 \\
\hline $\mathrm{C} 2$ & J02HP6 & $02 / 22 / 05$ & $3.38 \mathrm{E}-01$ & & 4.1E-02 & 1.5E-01 & U & 1.5E-01 & 1.4E-01 & $\mathrm{U}$ & 1.4E-01 \\
\hline D3 & J02HP7 & $02 / 22 / 05$ & $2.86 \mathrm{E}-01$ & & 3.0E-02 & 4.82E-01 & & $1 \mathrm{E}-02$ & & U & $.3 E-01$ \\
\hline D4 & J02HP8 & $02 / 22 / 05$ & $6.1 \mathrm{E}-02$ & & 3.6E-02 & $1.26 \mathrm{E}-01$ & & 8.1E-02 & $1.2 \mathrm{E}-01$ & $\mathrm{U}$ & $1.2 \mathrm{E}-01$ \\
\hline D5 & J02HP9 & $02 / 22 / 05$ & 1.05E-01 & & 3.7E-02 & 1.5E-01 & $\mathrm{U}$ & $1.5 E-01$ & 1.4E-01 & $\mathrm{U}$ & $1.4 \mathrm{E}-01$ \\
\hline D6 & J02HRO & $02 / 22 / 05$ & $1.52 E-01$ & & 3.5E-02 & 1.4E-01 & U & 1.4E-01 & 1.4E-01 & $u$ & 1.4E-01 \\
\hline$E 7$ & J02HR1 & $03 / 01 / 05$ & $1.08 \mathrm{E}-01$ & & 3.6E-02 & $2.65 E-01$ & & 1.0E-01 & 1.6E-01 & $U$ & 1.6E-01 \\
\hline E8 & J02HR2 & $03 / 01 / 05$ & $1.62 \mathrm{E}+00$ & & $5.0 \mathrm{E}-02$ & $1.24 \mathrm{E}+00$ & & 1.0E-01 & 2.3E-01 & $U$ & 2.3E-01 \\
\hline E9 & J02HR3 & $03 / 01 / 05$ & $1.36 \mathrm{E}-01$ & & 4.5E-02 & 4.65E-01 & & 1.0E-01 & $1.8 \mathrm{E}-01$ & $\mathrm{U}$ & 1.8E-01 \\
\hline E10 & J02HR4 & $03 / 01 / 05$ & $1.69 \mathrm{E}-01$ & & 4.5E-02 & 5.96E-01 & & 1.0E-01 & 1.7E-01 & U & 1.7E-01 \\
\hline $\mathrm{F} 1$ & J02HR5 & $03 / 01 / 05$ & $1.92 E-01$ & & $3.8 \mathrm{E}-02$ & $4.08 \mathrm{E}-01$ & & 7.8E-02 & $1.4 \mathrm{E}-01$ & $U$ & 1.4E-01 \\
\hline $\mathrm{F} 2$ & J02HR6 & $03 / 01 / 05$ & 3.6E-02 & & 3.6E-02 & $1.0 \mathrm{E}-01$ & $\mathrm{U}$ & 1.0E-01 & 1.3E-01 & U & 1.3E-01 \\
\hline F3 & J02HR7 & $03 / 01 / 05$ & 7.6E-02 & & 3.4E-02 & $1.2 \mathrm{E}-01$ & $\mathrm{U}$ & $1.2 \mathrm{E}-01$ & $1.5 \mathrm{E}-01$ & U & 1.5E-01 \\
\hline G5 & J02HTO & $03 / 02 / 05$ & 5.3E-02 & U & $5.3 \mathrm{E}-02$ & 1.3E-01 & $U$ & 1.3E-01 & $1.4 \mathrm{E}-01$ & $U$ & 1.4E-01 \\
\hline G6 & J02HT1 & $03 / 02 / 05$ & $3.6 \mathrm{E}-02$ & $U$ & 3.6E-02 & $8.6 \mathrm{E}-02$ & $U$ & $8.6 \mathrm{E}-02$ & $1.2 \mathrm{E}-01$ & $U$ & $1.2 \mathrm{E}-01$ \\
\hline $\mathrm{G} 7$ & $\mathrm{~J} 02 \mathrm{HT} 2$ & $03 / 02 / 05$ & 5.1E-02 & U & $5.1 \mathrm{E}-02$ & 1.1E-01 & $U$ & 1.1E-01 & $1.6 \mathrm{E}-01$ & $U$ & 1.6E-01 \\
\hline G8 & J02HT3 & $03 / 02 / 05$ & $2.58 \mathrm{E}-01$ & & 3.1E-02 & 5.63E-01 & & 7.2E-02 & 1.1E-01 & U & 1.1E-01 \\
\hline $\mathrm{H9}$ & $\mathrm{J} 02 \mathrm{HT} 4$ & $03 / 02 / 05$ & $2.06 \mathrm{E}-01$ & & 3.1E-02 & 3.33E-01 & & $6.0 \mathrm{E}-02$ & 9.7E-02 & $U$ & $9.7 \mathrm{E}-02$ \\
\hline $\mathrm{H} 10$ & J02HT5 & $03 / 02 / 05$ & 2.56E-01 & & 4.4E-02 & 1.91E-01 & & $8.2 \mathrm{E}-02$ & 1.2E-01 & $U$ & $1.2 \mathrm{E}-01$ \\
\hline $\mathrm{H1}$ & J02HT6 & $03 / 02 / 05$ & $1.40 \mathrm{E}-01$ & & 4.1E-02 & 7.60E-01 & & 7.3E-02 & 1.83E-01 & & 1.1E-01 \\
\hline $\mathrm{H} 2$ & J02HT7 & $03 / 02 / 05$ & 3.58E-01 & & $3.9 \mathrm{E}-02$ & 1.3E-01 & $U$ & 1.3E-01 & $1.5 \mathrm{E}-01$ & $U$ & 1.5E-01 \\
\hline 13 & J02HT8 & $02 / 18 / 05$ & $1.26 \mathrm{E}-01$ & & $3.0 \mathrm{E}-02$ & 1.1E-01 & U & 1.1E-01 & $1.4 \mathrm{E}-01$ & $U$ & 1.4E-01 \\
\hline 14 & J02HT9 & $02 / 18 / 05$ & 3.34E-01 & & 3.3E-02 & 1.2E-01 & $U$ & 1.2E-01 & $1.5 \mathrm{E}-01$ & $U$ & 1.5E-01 \\
\hline
\end{tabular}


Table A-5. Overburden Cleanup Verification Data. (2 Pages)

\begin{tabular}{|c|c|c|c|c|c|c|c|c|c|c|c|}
\hline \multirow{2}{*}{$\begin{array}{l}\text { Sampling } \\
\text { Area }\end{array}$} & \multirow{2}{*}{$\begin{array}{c}\text { HEIS } \\
\text { Number }\end{array}$} & \multirow{2}{*}{$\begin{array}{c}\text { Sample } \\
\text { Date }\end{array}$} & \multicolumn{3}{|c|}{ Cesium-137 } & \multicolumn{3}{|c|}{ Europium-152 } & \multicolumn{3}{|c|}{ Europium-154 } \\
\hline & & & $\mathrm{pCi} / \mathrm{g}$ & $\mathbf{Q}$ & MDA & $\mathrm{pCi} / \mathrm{g}$ & $\mathbf{Q}$ & MDA & $\mathrm{pCi} / \mathrm{g}$ & $\mathbf{Q}$ & MDA \\
\hline 15 & J02HVO & $02 / 18 / 05$ & $3.3 \mathrm{E}-02$ & $\mathrm{U}$ & 3.3E-02 & $8.1 \mathrm{E}-02$ & $U$ & $8.1 E-02$ & $1.2 \mathrm{E}-01$ & $U$ & $1.2 E-01$ \\
\hline 16 & J02HV1 & $02 / 18 / 05$ & 8.3E-02 & & $3.0 \mathrm{E}-02$ & 1.1E-01 & $\mathrm{u}$ & 1.1E-01 & 1.3E-01 & $\mathrm{U}$ & 1.3E-01 \\
\hline J7 & $\mathrm{J} 02 \mathrm{HV} 2$ & $02 / 17 / 05$ & $3.62 E-01$ & & $3.4 \mathrm{E}-02$ & 7.4E-02 & $\mathrm{U}$ & 7.4E-02 & $1.1 \mathrm{E}-01$ & $U$ & 1.1E-01 \\
\hline $\mathrm{J} 8$ & J02HV3 & $02 / 17 / 05$ & 5.1E-02 & & $2.0 \mathrm{E}-02$ & 4.3E-02 & & $5.0 \mathrm{E}-02$ & $7.8 \mathrm{E}-02$ & $U$ & $7.8 \mathrm{E}-02$ \\
\hline $\mathrm{J9}$ & $\mathrm{J} 02 \mathrm{HV} 4$ & $02 / 17 / 05$ & $1.51 \mathrm{E}-01$ & & $2.6 \mathrm{E}-02$ & $9.4 \mathrm{E}-02$ & $\mathrm{u}$ & $9.4 \mathrm{E}-02$ & 1.0E-01 & U & 1.0E-01 \\
\hline $\mathrm{J10}$ & J02HV5 & $02 / 17 / 05$ & 4.59E-01 & & 4.7E-02 & 2.55E-01 & & $9.4 \mathrm{E}-02$ & 1.7E-01 & $\mathrm{u}$ & 1.7E-01 \\
\hline $\mathrm{K} 1$ & J02HV6 & $02 / 17 / 05$ & 1.44E-01 & & 3.3E-02 & 8.3E-02 & $\mathrm{U}$ & 8.3E-02 & $1.2 \mathrm{E}-01$ & $U$ & $1.2 \mathrm{E}-01$ \\
\hline $\mathrm{K} 2$ & J02HV7 & $02 / 17 / 05$ & $3.1 \mathrm{E}-02$ & & $2.9 \mathrm{E}-02$ & 1.4E-01 & $\mathrm{U}$ & 1.4E-01 & 1.5E-01 & $\mathrm{U}$ & 1.5E-01 \\
\hline $\mathrm{K} 3$ & J02HV8 & $02 / 17 / 05$ & $4.2 \mathrm{E}-02$ & $U$ & $4.2 E-02$ & 1.0E-01 & $u$ & $1.0 \mathrm{E}-01$ & 1.6E-01 & $\mathrm{U}$ & $1.6 \mathrm{E}-01$ \\
\hline L.5 & J02HW1 & $02 / 17 / 05$ & $3.7 \mathrm{E}-02$ & $U$ & 3.7E-02 & $9.3 \mathrm{E}-02$ & $U$ & $9.3 \mathrm{E}-02$ & $1.3 \mathrm{E}-01$ & $\mathrm{U}$ & 1.3E-01 \\
\hline L6 & $\mathrm{J} 02 \mathrm{HW} 2$ & $02 / 17 / 05$ & 3.2E-02 & $\mathrm{U}$ & 3.2E-02 & 7.4E-02 & $U$ & $7.4 \mathrm{E}-02$ & $1.0 \mathrm{E}-01$ & $U$ & $1.0 \mathrm{E}-01$ \\
\hline L7 & J02HW3 & $02 / 17 / 05$ & 3.7E-02 & $U$ & 3.7E-02 & 1.3E-01 & $U$ & 1.3E-01 & 1.4E-01 & U & 1.4E-01 \\
\hline L8 & $\mathrm{J02HW4}$ & $02 / 17 / 05$ & $3.6 \mathrm{E}-02$ & $U$ & $3.6 \mathrm{E}-02$ & $9.3 E-02$ & $U$ & $9.3 \mathrm{E}-02$ & 1.3E-01 & $\mathrm{U}$ & 1.3E-01 \\
\hline
\end{tabular}


Table A-6. Discrete Samples Beneath the Former 100-K-55:1 and 100-K-56:1 Pipelines Within the Footprint of the 116-KW-3 and 116-KE-4 Retention Basins.

\begin{tabular}{|c|c|c|c|c|c|c|c|c|c|c|c|c|c|c|c|c|c|c|}
\hline \multirow{2}{*}{ HEIS No. } & \multicolumn{3}{|c|}{ Cesium-137 } & \multicolumn{3}{|c|}{ Cobalt- 60} & \multicolumn{3}{|c|}{ Europium-152 } & \multicolumn{3}{|c|}{ Europium-154 } & \multicolumn{3}{|c|}{ Plutonium-239/240 } & \multicolumn{3}{|c|}{ Strontium-90 } \\
\hline & $\mathrm{pCi} / \mathrm{g}$ & $Q$ & MDA & $\mathrm{pCi} / \mathrm{g}$ & $Q$ & MDA & $\mathrm{pCi} / \mathrm{g}$ & $Q$ & MDA & $\mathrm{pCi} / \mathrm{g}$ & $Q$ & MDA & $\mathrm{pCi} / \mathrm{g}$ & $Q$ & MDA & $\mathrm{pCi} / \mathrm{g}$ & $Q$ & MDA \\
\hline \multicolumn{19}{|c|}{$116-K W-3$} \\
\hline J02HX1 & $1.3 \mathrm{E}-01$ & & $5.8 \mathrm{E}-02$ & $6.0 \mathrm{E}-02$ & $u$ & $6.0 \mathrm{E}-02$ & $4.5 \mathrm{E}-01$ & & $1.0 \mathrm{E}-01$ & $2.0 \mathrm{E}-01$ & $u$ & $2.0 \mathrm{E}-01$ & $0.0 \mathrm{E}+00$ & $u$ & $1.6 \mathrm{E}-01$ & $1.19 \mathrm{E}-01$ & $U$ & $2.6 \mathrm{E}-01$ \\
\hline $\mathrm{J} 02 \mathrm{HX} 2$ & $6.1 \mathrm{E}-01$ & & $5.3 \mathrm{E}-02$ & $1.1 \mathrm{E}-01$ & & $3.1 \mathrm{E}-02$ & $1.3 E+00$ & & $9.6 \mathrm{E}-02$ & $1.9 \mathrm{E}-01$ & & $1.3 \mathrm{E}-01$ & $1.2 E-01$ & $\mathrm{U}$ & $1.8 \mathrm{E}-01$ & $3.66 \mathrm{E}-01$ & & $1.8 \mathrm{E}-01$ \\
\hline $\mathrm{J} 02 \mathrm{HX} 3$ & $4.2 \mathrm{E}-02$ & $u$ & $4.2 \mathrm{E}-02$ & $5.2 \mathrm{E}-02$ & $U$ & $5.2 \mathrm{E}-02$ & 1.3E-01 & $U$ & $1.3 \mathrm{E}-01$ & $1.8 \mathrm{E}-01$ & $u$ & $1.8 \mathrm{E}-01$ & $6.1 \mathrm{E}-02$ & $U$ & $1.6 \mathrm{E}-01$ & 1.57E-01 & $U$ & 1.7E-01 \\
\hline \multicolumn{19}{|c|}{$116-K E-4$} \\
\hline J030D3 & 2.3E-01 & & $5.6 \mathrm{E}-02$ & 1.5E-01 & & $5.0 \mathrm{E}-02$ & $1.93 E+00$ & & $1.1 \mathrm{E}-01$ & 3.3E-01 & & $1.8 \mathrm{E}-01$ & $2.4 \mathrm{E}-01$ & U & $6.2 \mathrm{E}-01$ & $2.38 \mathrm{E}-01$ & & 2.1E-01 \\
\hline J030D4 & $3.1 \mathrm{E}-01$ & & $8.2 \mathrm{E}-02$ & $1.2 \mathrm{E}-01$ & & $6.4 \mathrm{E}-02$ & $3.11 E+00$ & & 1.3E-01 & $3.3 \mathrm{E}-01$ & & 1.8E-01 & $7.9 \mathrm{E}-02$ & U & $3.0 \mathrm{E}-01$ & $4.96 \mathrm{E}-01$ & & $2.5 E-01$ \\
\hline J030D5 & $6.1 \mathrm{E}-02$ & & $4.5 \mathrm{E}-02$ & $5.6 \mathrm{E}-02$ & & $4.9 \mathrm{E}-02$ & $6.08 \mathrm{E}-01$ & & $8.8 \mathrm{E}-02$ & $1.5 \mathrm{E}-01$ & $u$ & $1.5 \mathrm{E}-01$ & $3.6 \mathrm{E}-02$ & $u$ & $2.8 \mathrm{E}-01$ & $4.32 E-01$ & & 2.2E-01 \\
\hline
\end{tabular}

\begin{tabular}{|c|c|c|c|c|c|c|c|c|c|}
\hline \multirow{2}{*}{ HEIS No. } & \multicolumn{3}{|c|}{ Uranium-233/234 } & \multicolumn{3}{|c|}{ Uranium-235 } & \multicolumn{3}{|c|}{ Hexavalent Chromium } \\
\hline & $\mathrm{pCi} / \mathrm{g}$ & $Q$ & MDA & $\mathrm{pCi} / \mathrm{g}$ & $\mathbf{Q}$ & MDA & $\mathrm{mg} / \mathrm{kg}$ & $Q$ & PQL \\
\hline \multicolumn{10}{|c|}{$116-K W-3$} \\
\hline $\mathrm{J} 02 \mathrm{HX} 1$ & 7.0E-01 & & $1.4 \mathrm{E}-01$ & $4.5 \mathrm{E}-02$ & & 1.7E-01 & $2 E-01$ & $U$ & $2 E-01$ \\
\hline $\mathrm{J} 02 \mathrm{H} \times 2$ & $5.1 \mathrm{E}-01$ & & 1.4E-01 & $4.4 \mathrm{E}-02$ & & 1.7E-01 & $2 E-01$ & $\mathrm{U}$ & $2 \mathrm{E}-01$ \\
\hline $\mathrm{J} 02 \mathrm{HX} 3$ & $6.2 \mathrm{E}-01$ & & $1.5 \mathrm{E}-01$ & $9.4 \mathrm{E}-02$ & & $1.8 \mathrm{E}-01$ & $2 E-01$ & $u$ & $2 \mathrm{E}=01$ \\
\hline \multicolumn{10}{|c|}{ 116-KE-4 } \\
\hline Jo30D3 & $3.80 \mathrm{E}-01$ & & 1.7E-01 & $2.7 \mathrm{E}-02$ & & 2.1E-01 & 2.2E-01 & $\mathrm{U}$ & 2.2E-01 \\
\hline J030D4 & $3.94 \mathrm{E}-01$ & & $2.0 \mathrm{E}-01$ & $0.0 \mathrm{E}+00$ & $\mathrm{u}$ & $2.4 \mathrm{E}-01$ & $2.4 \mathrm{E}-01$ & & $2.1 \mathrm{E}-01$ \\
\hline Jo30D5 & $4.54 \mathrm{E}-01$ & & $2.3 \mathrm{E}-01$ & $0.0 \mathrm{E}+00$ & $u$ & $2.8 \mathrm{E}-01$ & $9.4 \mathrm{E}-01$ & & 2.1E-01 \\
\hline
\end{tabular}


Table A-7. Discrete Samples Beneath the Former Locations of the Heat Recovery Stations.

\begin{tabular}{|c|c|c|c|c|c|c|c|c|c|c|c|c|c|c|}
\hline \multirow{2}{*}{ HEIS No. } & \multicolumn{2}{|c|}{ Arsenic } & \multicolumn{2}{|c|}{ Barium } & \multicolumn{3}{|c|}{ Cadmium } & \multicolumn{2}{|c|}{ Total Chromium } & \multicolumn{2}{|c|}{ Mercury } & \multicolumn{3}{|c|}{ Lead } \\
\hline & $\mathrm{mg} / \mathrm{kg}$ & \begin{tabular}{|l|l|}
$Q$ & PQL \\
\end{tabular} & $\mathrm{mg} / \mathrm{kg}$ & PQL & $\mathrm{mg} / \mathrm{kg}$ & Q & PQL & $\mathbf{m g} / \mathbf{k g}$ & PQL & $\mathrm{mg} / \mathrm{kg}$ & PQL & $\mathrm{mg} / \mathrm{kg}$ & $Q$ & PQL \\
\hline \multicolumn{15}{|c|}{$116-K W-4$} \\
\hline $\mathrm{J} 02 \mathrm{HX} 4$ & $2.6 \mathrm{E}+00$ & $2.9 \mathrm{E}-01$ & $5.93 E+01$ & 2.0E-02 & 1.6E-01 & $\mathrm{U}$ & $1.6 \mathrm{E}-01$ & $1.21 \mathrm{E}+01$ & $1.6 \mathrm{E}-01$ & $2 \mathrm{E}-02$ & $1 E-02$ & $4.5 E+00$ & & 7.6E-01 \\
\hline \multicolumn{15}{|c|}{$116-K E-5$} \\
\hline J030D6 & $3.7 E+00$ & 3.0E-01 & $1.0 E+02$ & $2.0 \mathrm{E}-02$ & $1.9 E-01$ & $\mathrm{U}$ & $4.0 E-02$ & $3.0 E+01$ & 4.0E-02 & 1.0E-01 & 2.0E-02 & $1.1 E+01$ & & $1.9 \mathrm{E}-01$ \\
\hline
\end{tabular}

\begin{tabular}{|c|c|c|c|c|c|c|}
\hline \multirow{2}{*}{ HEIS No. } & \multicolumn{3}{|c|}{ Hexavalent Chromium } & \multicolumn{3}{|c|}{ Ethylene Glycol } \\
\hline & $\mathrm{mg} / \mathrm{kg}$ & $Q$ & PQL & $\mathbf{m g} / \mathbf{k g}$ & $\mathbf{Q}$ & PQL \\
\hline \multicolumn{7}{|c|}{$116-K W-4$} \\
\hline $\mathrm{JO} 2 \mathrm{H} \times 4$ & $2.4 \mathrm{E}-01$ & & 2.1E-01 & $1.0 E+01$ & $\mathrm{U}$ & $1.0 \mathrm{E}+01$ \\
\hline \multicolumn{7}{|c|}{ 116-KE-5 } \\
\hline J030D6 & 3.3E-01 & & 2.1E-01 & $5.9 E+01$ & & $1.0 E+01$ \\
\hline
\end{tabular}


CVP-2005-00006

Rev. 0

A-8 
CVP-2005-00006

Rev. 0

APPENDIX B

\section{DATA QUALITY ASSESSMENT}


CVP-2005-00006

Rev. 0

B-ii 
CVP-2005-00006

Rev. 0

\section{B1.0 DATA QUALITY ASSESSMENT FOR THE 100-K-55:1 AND 100-K-56:1 PIPELINES AND THE 116-KW-4 AND 116-KE-5 HEAT RECOVERY STATIONS}

\section{B1.1 OVERVIEW}

The data quality assessment (DQA) completes the data life cycle (i.e., planning, implementation, and assessment) that was initiated by the data quality objectives process. The DQA includes a review of the field logbook information ( $\mathrm{BHI} 2005 \mathrm{a}, 2005 \mathrm{~b})$ to verify sample location, date, and time. It also involves the scientific and statistical evaluation of the data to determine if they are of the right type, quality, and quantity to support their intended use for closeout decisions (EPA 2000).

This DQA was performed in accordance with BHI-EE-01, Environmental Investigations Procedures. Specific data quality objectives for the site are found in the 100 Area Remedial Action Sampling and Analysis Plan (SAP) (DOE-RL 2005a). The DQA is based on the guidelines presented in Guidance for Data Quality Assessment (EPA 2000). Statistical tests used in this DQA were performed as specified in the SAP and the Remedial Design Report/Remedial Action Work Plan for the 100 Area (RDR/RAWP) (DOE-RL 2005b).

Prior to performing statistical tests, the field logbooks (BHI 2005a, 2005b), sample designs (Appendix C), and sample analytical data are evaluated. A portion of the cleanup verification sample analytical data are validated for compliance requirements (DOE-RL 2005a). Data evaluation is performed to determine if the laboratory carried out all steps required by the SAP and the laboratory contract governing the conduct of analysis and reporting of the data. This evaluation also examines the available laboratory data to determine if an analyte is present or absent in a sample and the degree of overall uncertainty associated with that determination. Data validation is done in accordance with validation procedures (BHI 2000a, 2000b) as part of data evaluation. After data evaluation and validation, the appropriate statistical test is performed on the adjusted raw analytical data (Appendix $\mathrm{C}$ ) to determine statistical values for each contaminant. The cleanup verification sample analytical data are stored in the Hanford Environmental Information System and are summarized in Appendix A.

For the 100-K-55:1, 100-K-56:1, 116-KW-4, and 116-KE-5 sites, 140 samples in 20 sample delivery groups (SDGs) have been evaluated in the following sections.

\section{B1.2 LABORATORY QUALITY MEASURES}

All verification samples are subject to laboratory-specific quality assurance (QA) requirements, including instrument procurement, maintenance, calibration, and operation. Additional laboratory quality control (QC) checks are performed, as 
appropriate, for the analytical method at a rate of 1 per SDG, or 1 in 20 , whichever is more frequent. Laboratory internal QC checks include the following:

- Laboratory Contamination. Each analytical batch contains a laboratory (method) blank (material of similar composition as the samples with known/minimal contamination of the analytes of interest) carried through the complete analytical process. The method blank is used to evaluate false-positive results in samples due to contamination during handling at the laboratory.

- Analytical Accuracy. For most analyses, a known quantity of representative analytes of interest (matrix spike [MS]) is added to a separate aliquot of a sample from the analytical batch. The recovery percentage of the added MS is used to evaluate analytical accuracy. For analyses not amenable to MS techniques (e.g., gamma energy analysis) or where analytical recovery is corrected via internal standards (e.g., alpha spectral analyses), accuracy is evaluated from recovery of the QC reference sample (e.g., laboratory control spike or blank spike sample).

- Analytical Precision. Separate aliquots removed from the same sample container (replicate samples) are analyzed for each analytical batch. The replicate sample results (evaluated as relative percent differences [RPDs]) are used to assess analytical precision.

- QC Reference Samples. A QC reference sample is prepared from an independent standard at a concentration other than that used for calibration, but within the calibration range. Reference samples provide an independent check on analytical technique and methodology.

Laboratories are also subject to periodic and random assessments of the laboratory performance, systems, and overall program. These assessments are performed by the Washington Closure Hanford QA group to ensure that the laboratories are performing within laboratory contract requirements.

\section{B1.3 DATA VALIDATION}

After sampling was completed, all of the fixed-base laboratory data from two SDGs (H2997 and H3218), were validated by a third-party validator to Level C per BHI-EE-01, Procedure 2.5, "Data Package Validation Process." Level $\mathrm{C}$ validation procedures are specified in Data Validation Procedure for Radiochemical Analysis (BHI 2000b) and Data Validation Procedure for Chemical Analysis (BHI 2000a).

Use of level $\mathrm{C}$ validation procedures were included in the review of the following items, as appropriate, for each analytical method:

- Sample holding times

- Method blanks

- MS recovery 
- Surrogate recovery

- MS/matrix spike duplicate results

- Sample replicates

- Associated batch laboratory control sample results

- Data package completeness

- Achievement of required (or contractual) detection limits (RDLs).

Data flagged by the validator as estimated (i.e., "J") indicate that the associated concentration is an estimate, but that the data may be used for decision-making purposes. Data flagged as below detection limits (i.e., "U") indicate the contaminant was analyzed for but not detected, and the concentration is below the minimum detectable activity (MDA) for radionuclides or the practical quantitation limit (PQL) (i.e., reporting limit) for nonradionuclides. For nonradionuclides, nondetects are reported as the PQL. For radionuclides, nondetects report the actual value obtained from analysis (positive or negative but less than the MDA) except for limited analyses where no value can be calculated. In these cases, the MDA is reported. This situation is applicable for sample results that are below detection limits. All other validated results are considered to be accurate within the standard errors associated with the methods.

The adequacy of laboratory QA/QC was evaluated for precision, accuracy, completeness, and RDLs pursuant to the SAP (DOE-RL 2005a). The organization performing the data validation reported that, of the data given formal validation, the laboratory met the standards for performance for precision $( \pm 30 \%)$, accuracy $( \pm 30 \%)$, and completeness $(>90 \%)$. Comparison of the RDL with the respective MDA or PQL is discussed in Section B1.4.

The validated SDG H2997 contains 10 samples $(\mathrm{J02H07,} \mathrm{J02H08,} \mathrm{J02H09,} \mathrm{J02H10,}$ $\mathrm{J} 02 \mathrm{H} 11, \mathrm{J02} \mathrm{H} 012, \mathrm{J02H} 13, \mathrm{J02H} 14, \mathrm{J02H} 15$, and $\mathrm{J02H} 16$ ). A summary of deficiencies noted during validation of SDG $\mathrm{H} 2997$ follows.

- Radionuclides. The validation DQA noted no major deficiencies.

Because of a high RPD in the duplicate analysis for thorium-228 (54\%), all thorium-228 results were qualified as estimates and flagged "J." Data flagged "J" indicate that the associated concentration is an estimate, but the data are usable for decision-making purposes. All other validated results are considered accurate within the standard error associated with the methods.

- Nonradionuclides. SDG H2997 did not contain nonradionuclide analysis.

The validated SDG H3218 contains 10 samples (J037K9, J037L0, J037L1, J037L2, J037L3, J037L4, J037L5, J037L6, J037L7, and J037L8). A summary of deficiencies noted during validation of SDG H2997 follows. 
- Radionuclides. The validation DQA noted no major deficiencies.

Because of a high RPD in the duplicate analysis for thorium-228 (38\%), all thorium-228 results were qualified as estimates and flagged "J." Data flagged "J" indicate that the associated concentration is an estimate, but the data are usable for decision-making purposes. All other validated results are considered accurate within the standard error associated with the methods.

- Nonradionuclides. SDG H3218 did not contain nonradionuclide analysis.

\section{B1.4 LABORATORY DATA EVALUATION}

The following paragraphs include the results of the data evaluation of 20 verification sample SDGs.

The context for assessing the data includes evaluating the sample data using the statistical methodology of the SAP (DOE-RL 2005a) (included in the calculation brief excerpts in Appendix C) and a comparison of analytical results to the parameters as specified in the SAP. This section summarizes the results of the comparison and presents an evaluation of the affected data.

Sample Holding Times. All of the method-specific holding times were met for all samples.

Method Blanks. The method blank is used to evaluate false-positive results in samples due to contamination during handling at the laboratory.

Radionuclides. No target analytes were detected in the method blanks.

Nonradionuclides. In the method blank for SDG H3032, barium was detected at $0.04 \mathrm{mg} / \mathrm{kg}$. The RDL for barium is $2.0 \mathrm{mg} / \mathrm{kg}$ and the low-level detection in the method blank does not compromise any sample data.

In the method blank for SDG H3112, barium was detected at $0.12 \mathrm{mg} / \mathrm{kg}$ and total chromium was detected at $0.06 \mathrm{mg} / \mathrm{kg}$. The RDLs for these analytes are $2.0 \mathrm{mg} / \mathrm{kg}$ and $1.0 \mathrm{mg} / \mathrm{kg}$, respectively. There is no impact to sample data from these detections in the method blank.

RDL Comparison. Reported analytical detection levels for nondetected analytes were compared to the RDLs specified in the SAP (DOE-RL 2005a). When detected results were obtained, evaluation of detection limits was not performed. The data validation and supplemental data evaluation noted any analyses in which the detection limit (MDA or PQL) was above the SAP RDLs for nondetected analytes. 
Radionuclides. All of the reported MDAs are sufficiently less than the remedial action goals (RAGs), and the data are of sufficient quality for decision-making purposes. All values meet applicable RAGs as demonstrated in the calculation briefs (Appendix C) and discussed in this cleanup verification package.

Nonradionuclides. All of the reported method detection limits are less than applicable RAGs, and the data are of sufficient quality for decision-making purposes.

Precision and Accuracy Evaluation. Analytical accuracy and precision were evaluated by examination of the RPD of the main and duplicate samples. Only the contaminants of concern (COCs) detected at five times the detection limit (or greater) are used for data analysis with respect to accuracy and precision.

Radionuclides. In SDG H3067, the duplicate analysis produced four analytes whose RPDs were above acceptance criteria (cesium-137 [155\%], radium-228 [55\%], europium-154 [60\%], and europium-155 [55\%]). The high RPDs observed in this SDG were for analytes that were detected at relatively low levels where sample heterogeneity has more impact on RPD analysis.

In SDG H3218, the duplicate analysis for thorium-228 had an RPD above the acceptance criteria at $38 \%$. Third-party validation was performed on SDG H3218 and resulted in all of the associated thorium-228 results being qualified as estimates with "J" flags.

Duplicate samples are produced using field collected materials. The natural heterogeneity of these materials adds to the elevated RPDs of the duplicate analysis. This variability is expected and does not indicate a problem with the analytical system. RPDs of analytes detected at low concentrations (less than five times the detection limit) are also not considered to be indicative of the analytical system performance. Where elevated RPDs are observed, it is typical to consider the data to be estimated as the validator has done in SDG H3218. Estimated data is useable for decision-making purposes.

\section{B1.5 FIELD QUALITY ASSURANCE/QUALITY CONTROL}

Field QA/QC measures were used to assess potential sources of error and cross contamination of soil samples that could bias results. Field QA/QC samples listed in the field logbooks (BHI 2005a, 2005b) are summarized in Table B-1. All main and QA/QC sample results are presented in Appendix A. 
Table B-1. Summary of Field Quality Control Samples.

\begin{tabular}{|c|c|c|c|c|}
\hline Sample Area & Main Sample & Equipment Blank $^{\text {a }}$ & Duplicate & Split \\
\hline A1 & J03D45 & NA & J03D46 & J03D86 \\
\hline A1 & J02H20 & NA & J02HW9 & J02HX0 \\
\hline A4 & J02HK9 & NA & J02HL0 & J02HN2 \\
\hline A4 & J02HN7 & NA & J02HN8 & J02HW5 \\
\hline C9 & J037L7 & NA & J037L8 & J037L9 \\
\hline D2 & J02H14 & J02H15 & J02H15 & J02H21 \\
\hline F4 & J02HR8 & NA & J02HR9 & J02HW6 \\
\hline F4 & J025N0 & NA & J025N1 & J025N3 \\
\hline K4 & J02HV9 & NA & J02HW0 & J02HW7 \\
\hline
\end{tabular}

${ }^{a}$ The sampling plans did not call for equipment blanks.

$\mathrm{NA}=$ not applicable

A single equipment blank sample was collected, sample J02H15. The equipment blank was analyzed by gamma spectroscopy for potassium- 40 , cobalt- 60 , cesium-137, radium-226, radium-228, europium-152, europium-154, europium-155, thorium-228, thorium-232, uranium-235, uranium-238, and americium-241. Low-level detections for potassium-40, radium-226, radium-228, thorium-228, and thorium-232 were observed. All of the detected analytes are significantly below cleanup levels. The data is suitable for the intended purpose of demonstrating cleanup verification and no further qualification is required.

Field duplicate samples were collected to provide a relative measure of the degree of local heterogeneity in the sampling medium, unlike laboratory duplicates that are used to evaluate precision in the analytical process. The field duplicates are evaluated by computing the RPD of the duplicate samples for each COC. Only analytes with values above five times the detection limits for both the main and duplicate samples are compared. Only one duplicate pair produced an elevated RPD. The duplicate pair $\mathrm{J} 02 \mathrm{HK} / \mathrm{JO2HLO}$ had an RPD of $47 \%$ for europium-152. This result is typical of the heterogeneity found in the sample matrixes and does not indicate a problem with the analytical system. All other field duplicate results were acceptable. The data are suitable for the intended purpose of cleanup verification. The $95 \%$ upper confidence limit (UCL) calculation briefs in Appendix $\mathrm{C}$ provide details on duplicate pair evaluation and RPD calculation.

Split samples were collected to provide a relative measure of the degree of variability in the sampling, sample handling, and analytical techniques used by commercial laboratories. The field main and split samples are evaluated by computing the RPD of the split samples for each $\mathrm{COC}$ to determine the usability of the verification data. The U.S. Environmental Protection Agency Contract Laboratory Program duplicate sample comparison methodology, USEPA Contract Laboratory Program National Functional Guidelines for Inorganic Data Review (EPA 1994), is used as an initial test of the data 
from the splits. Only analytes that had values above five times the contractual RDL for both the main and split sample were compared. The RPD calculation produced three instances of elevated RPDs. The split pair J02HK9/J02HN2 had an RPD of $63 \%$ for europium-152. The split pair J037L7/J037L9 had an RPD of $47.5 \%$ for cesium-137 and $51.3 \%$ for europium-152. These results are typical of the heterogeneity found in the sample matrices and does not indicate a problem with either of the laboratories analytical systems. All other split sample pairs are within acceptable control limits. The 95\% UCL calculation briefs in Appendix C provide details on split pair RPD calculation.

\section{B1.6 SUITABILITY OF DATA}

The DQA for the 100-K-55:1, 100-K-56:1, 116-KW-4, and 116-KE-5 sites determined that the data are of the right type, quality, and quantity to support site cleanup verification decisions within specified error tolerances. The evaluation verified that the sample design was sufficient for the purpose of clean site verification. All analytical data were found to be acceptable for decision-making purposes, and the raw data are acceptable for calculating the required statistical values.

\section{B2.0 REFERENCES}

BHI, 2000a, Data Validation Procedure for Chemical Analysis, BHI-01435, Rev. 0, Bechtel Hanford, Inc., Richland, Washington.

BHI, 2000b, Data Validation Procedure for Radiochemical Analysis, BHI-01433, Rev. 0, Bechtel Hanford, Inc., Richland, Washington.

BHI, 2005a, 100K Remedial Sampling Logbook, EL-1572-2, Bechtel Hanford, Inc., Richland, Washington.

BHI, 2005b, 100K Remedial Sampling Logbook, EL-1572-3, Bechtel Hanford, Inc., Richland, Washington.

BHI-EE-01, Environmental Investigations Procedures, Bechtel Hanford, Inc., Richland, Washington.

DOE-RL, 2005a, 100 Area Remedial Action Sampling and Analysis Plan, DOE/RL-96-22, Rev. 4, U.S. Department of Energy, Richland Operations Office, Richland, Washington.

DOE-RL, 2005b, Remedial Design Report/Remedial Action Work Plan for the 100 Area, DOE/RL-96-17, Rev. 5, U.S. Department of Energy, Richland Operations Office, Richland, Washington. 
EPA, 1994, USEPA Contract Laboratory Program National Functional Guidelines for Inorganic Data Review, EPA 540/R-94/013, U.S. Environmental Protection Agency, Washington, D.C.

EPA, 2000, Guidance for Data Quality Assessment, EPA QA/G-9, QA00 Update, U.S. Environmental Protection Agency, Office of Environmental Information, Washington, D.C. 
APPENDIX C

RESRAD INPUT PARAMETERS

AND CALCULATION BRIEF EXCERPTS 
CVP-2005-00006

Rev. 0 
CVP-2005-00006

Rev. 0

\section{RESRAD INPUT PARAMETERS FOR THE} 100-K-55:1 SHALLOW ZONE 
CVP-2005-00006

Rev. 0 
CVP-2005-00006

Rev. 0

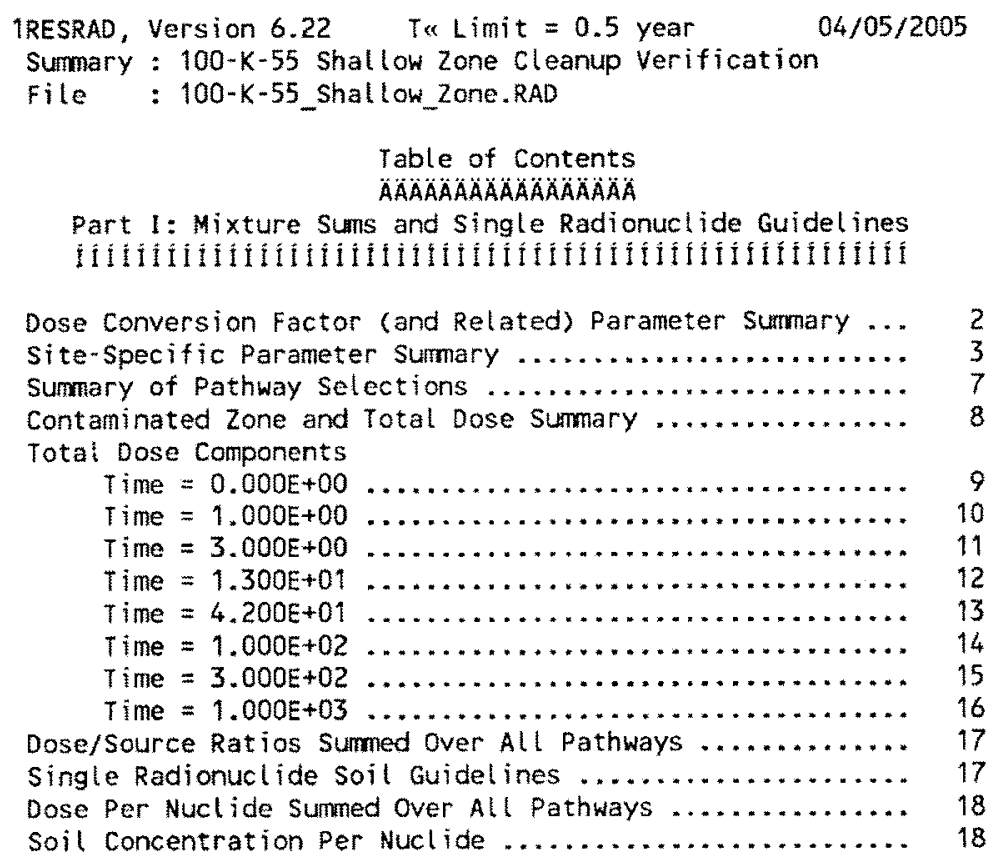




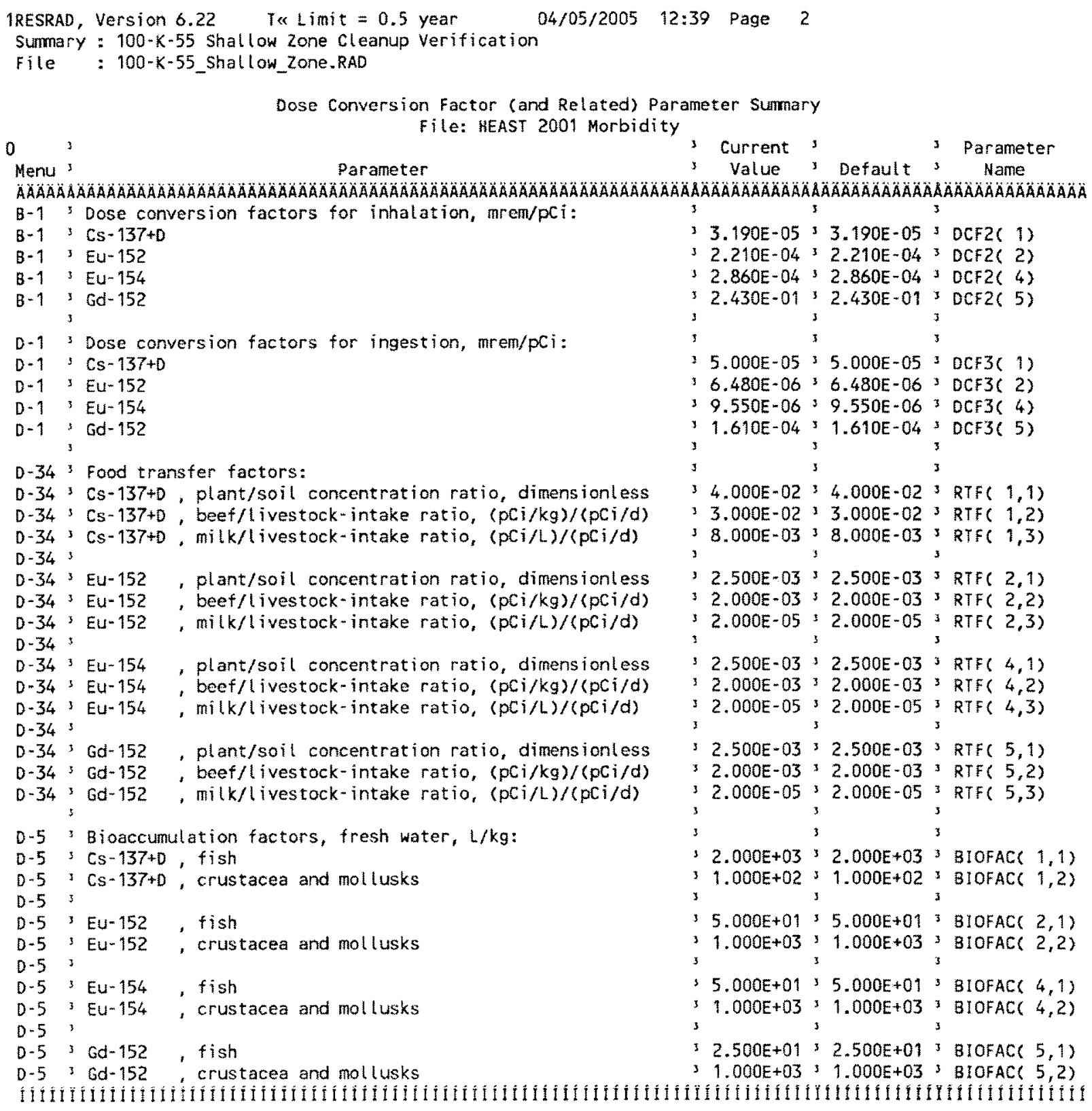


CVP-2005-00006

Rev. 0

1RESRAD, Version 6.22 T\& Limit $=0.5$ year $04 / 05 / 2005 \quad 12: 39$ Page 3 Sumary : $100-\mathrm{K}-55$ shallow Zone cleanup Verification

File: $100-k-55$ shallow Zone. RAD

0

Site-Specific Parameter Sumary

Menu 3 Parameter

-User,

Used by RESRAD

3 Parameter

R011; Area of contaminated zone $\left(\mathrm{m}^{* *} 2\right)$

R011 : Thickness of contaminated zone $(\mathrm{m})$

R011' Length parallel to aquifer flow (m)

R011 Basic radiation dose linit (mrenyr)

8011 T Tines for calculations ( $y r$ )

R011 ? Times for calculations $(y r)$

Ro11 Tines for calcutations (yr)

Ro11: Times for calculations (yr)

R011' Times for calculations (yr)

R012, Initial principal radionuclide $(\mathrm{pCi} / \mathrm{g}): \mathrm{Cs}-137,2.710 \mathrm{~F}-01,0.000 \mathrm{E}+00$

R012 Initial principal radionuclide $(\mathrm{pCi} / 9)$ : Eu-152, $7.270 \mathrm{E}-01,0.000 \mathrm{E}+00$;

$\mathrm{R} 012$ I Initial principal radionuclide $(\mathrm{pCi} / \mathrm{g})$ : Eu-154, $1.200 \mathrm{E}-0130.000 \mathrm{E}+00$

0123 Concentration in groundwater (PCi/L): $\mathrm{Cs}_{2}-137$ not used $30.000 \mathrm{E}+00$

(pci/L): Eu-152 not used $30.000 E+00$

(pCi/l):

(pci/h): Eu-154

R013' Cover depth (m)

2013 : Density of cover material ( $/ \mathrm{cm}^{* * 3}$ )

R013 Cover depth erosion rate (m/yr)

R013' Density of contaminated zone $(\mathrm{g} / \mathrm{cm} * * 3)$

R013' Contaminated zone erosion rate (m/yr)

R013 Contaminated zone cotal porosity

8013 * Contaminated zone field capacity

R013" Contaminated zone hydraulic conductivity (m/yr)

R013' Contaminated zone b parameter

R013' Average annual wind speed $(\mathrm{m} / \mathrm{sec}\rangle$

R013' Hunidity in air $\left(9 / \mathrm{m}^{* * 3}\right)$

R013 S Evapotranspiration coefficient

R013 Precipitation (m/yr)

R013 * Irrigation (m/yr)

R013 3 Irrigation mode

R013: Runot coefficient

R013 Watershed area for nearby strean or pond $\left(m^{* * 2)}\right.$

R013 3 Accuracy for water/soil computations

R014 ${ }^{3}$ Density of saturated zone $\left(\mathrm{g} / \mathrm{cm}^{* * 3}\right)$

Ro14 3 Saturated zone total porosity

R014 3 Saturated zone effective porosity

Ro14 3 Saturated zone field capacity

RO14 Saturated zone hydraulic conductivity (m/yr)

R014 3 Saturated zone hydraulic gradient

R014 3 Saturated zone b parameter

RO14 3 water table drop rate ( $m / y r)$

Ro14 We!l pump intake depth (m below water table)

$\$ 0.000 E+00^{3} 0.000 E+00$

not used $39.500 \mathrm{E}+00$

3 not used $1.000 E-03$

$31.600 E+00 ; 1.500 E+00$,

$1.000 \mathrm{E}-03: 1.000 \mathrm{E}-03$

$4.000 E-01+4.000 E-01$

2.000E-01;2.000E-01

$12.500 E+02 \div 1.000 E+01$

$4.050 E+00 ; 5.300 E+00$

$3.400 E+00>2.000 E+00$

not used $8.000 E+00$

$39.100 E-01 ; 5.000 E-01$

$31.600 E-0131.000 E+00$

$7.600 E-01 ; 2.000 E-01$

overhead 3 overhead

2.000 - $01 ; 2.000$ E-01

$1.000 E+0631.000 E+06$

$31.000 E-03+1.000 E-03 s$

$1.600 \mathrm{E}+00 ; 1.500 \mathrm{E}+00$

$4.000 E-01+4.000 E-01$

3 2.00E-01:2.000E-01

3. $1.500 \mathrm{E}-01 ; 2.000 \mathrm{E}-01$ :

$5.530 E+03 ; 1.000 E+02$

$31.250 \mathrm{E}-03 ; 2.000 \mathrm{E}-02$

$4.050 E+0035.300 E+00$

$3,000 E-03: 1.000 E-03$

$3.600 E+00 \times 1.000 E+01=$
$2.498 E+04,1.000 E+04$

$32.220 E+01 \div 2.000 E+00$

$32.040 E+02 \times 1.000 E+02$

$31.500 E+01 ; 2.500 E+01$

$=0.000 E+00>0.000 E+00$

$1.000 E+00,1.000 E+00$

$3.000 E+003.000 E+00$

$31.300 E+01 ; 1.000 E+01$

$4.200 E+0133.000 E+01$

$11.000 E+02 ; 1.000 E+02$

$33.000 E+02 ; 3.000 E+02$

$31.000 E+03,1.000 E+03$

not used $10.000 E+00$

not used $0.000 E+00$

$\begin{array}{ll}\ldots & \\ \ldots & :\end{array}$

...

3 TI

$\cdots \quad$ s $T(2)$

... 3 T( 3$)$

...

$3 T(5)$

$=T(6)$

$\ldots \quad 3$ T( 7$)$

$\cdots+1(8)$

-.. 3 T 9 ( 9$)$

i $T(10)$

n.. $\quad$ sik 1

$\ldots+\operatorname{sic} 2)$

$\ldots+51(4)$

3 W1 18

3 W1( 2$)$

...
1 COVERO

DENSCV

" VCV

- DENSCZ

VCZ

- $\mathrm{TPCZ}$

: Fecz

HCCZ

BCZ

WIND

3 HUMID

EVAPTR

PRECIP

$\mathrm{RI}$

IDITCH

RUNOFF

WAREA

EPS

DENSAQ

IPSZ

EPSZ

F FCSZ

- HCSR

3 HGW

BS2 
CVP-2005-00006

Rev. 0

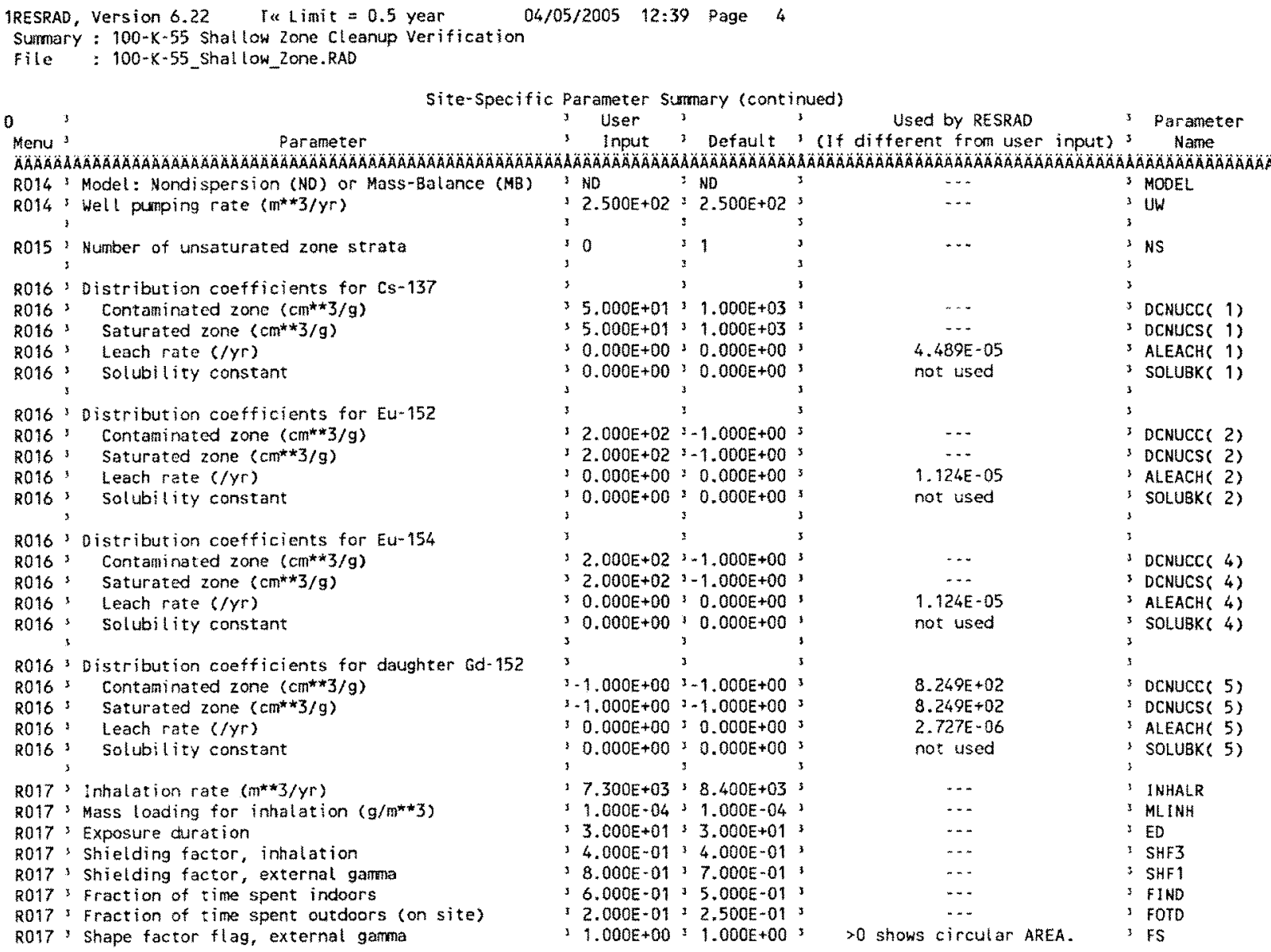


1RESRAD, Version 6.22 Tu Limit $=0.5$ year 04/05/2005 12:39 Page 5

Sumary: $100-k-55$ Shallow zone Cleanup Verification

File: $100-k \cdot 55$ shallow_zone.RAD

Site-Specific Parameter Sumary (continued)

"senus

Parameter

3 User 3

Used by RESRAD

3 Parameter

Menu " Parameter " Input " Default (If different from user input) " Name

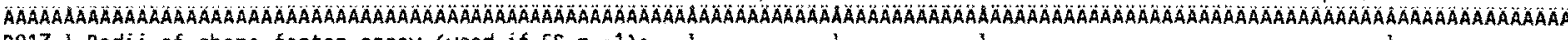
R017 3 Radi $i$ of shape factor array (used if $F S=-1):$

R017' Outer annular radius (m), ring 1 ;

R0173 Outer annular radius (m), ring 2:

R017, Outer antular radius $(\mathrm{m})$, ring 3 ;

' not used? $5.000 \mathrm{E}+01^{3}$

not used $37.071 \mathrm{E}+01$

3 not used $\$ 0.000 \mathrm{E}+00$

3 not used $\div 0.000 E+00$,

not used, $0.000 \mathrm{E}+00$

not used $0.000 E+00$

not used : $0.000 \mathrm{E}+00$

3 not used $: 0.000 E+00$

not used $30.000 E+00$ ?

not used $30.000 \mathrm{E}+00$ ?

not used $+0.000 E+00$

not used $30.000 E+00$

not used: $1.000 \mathrm{E}+00$

not used $32.732 E-01$

not used $: 0.000 E+00$

3 not used $=0.000 \mathrm{E}+00$

not used $=0.000 \mathrm{E}+00^{3}$

not used $=0.000 \mathrm{E}+00$

not used $=0.000 \mathrm{E}+00^{3}$

not used $: 0.000 \mathrm{E}+00$

3 not used $20.000 E+00$

3 not used $: 0.000 \mathrm{E}+00^{3}$

not used $=0.000 E+00$ ?

not used $: 0.000 \mathrm{E}+00^{3}$

Ring 12

R018 "Fruits, vegetables and grain consumption ( $\mathrm{kg} / \mathrm{yr})$ "

R018' Leafy vegetable consumption (kg/yr)

R018: Milk consumption ( $L / Y$ r )

R018 + Meat and poultry consumption ( $\mathrm{kg} / \mathrm{yr}$ )

R018 3 Fish consumotion $(\mathrm{kg} / \mathrm{Yr})$

R018 : Other sesfood consumption ( $\mathrm{kg} / \mathrm{yr}$ )

RO18 3 Soil ingestion rate $(g / y r)$

Ro18' Orinking water intake $(\mathrm{L} / \mathrm{Yr}$

R018 3 Contamination fraction of drinking water

R018 3 Contanination fraction of household water

R018 3 Contanination fraction of livestock water

R018 * Contamination fraction of irrigation water

Rot8' Contamination fraction of aquatic food

R018 3 Contamination fraction of plant food

R018 3 Contamination fraction of meat

Ro18 " Contamination fraction of milk

R019? Livestock fodder intake for meat ( $\mathrm{kg} /$ day)

R019 3 Livestock fodder intake for $n i l \mathrm{k}$ ( $\mathrm{kg} /$ day)

R019 3 Livestock water intake for meat (L/day)

Rot9: kivestock water intake for milk (L/day)

R019. Livestock soll intake (kg/day)
$02 \div 1.600 E+02$

$2.700 \mathrm{E}+00=1.400 \mathrm{E}+01$

$1.000 \mathrm{E}+02^{3} 9.200 \mathrm{E}+01$

$3.600 E+01: 6.300 E+01$

$1.970 \mathrm{E}+01 ; 5.400 \mathrm{E}+00$ ?

$9.000 \mathrm{E}-01: 9.000 \mathrm{E}-01$

$7.300 E+0133.650 E+01$

$7.300 \mathrm{E}+02,5.100 \mathrm{E}+02$

$1.000 E+00 ; 1.000 E+00$

not used $1.000 E+00$

$31.000 E+0031.000 E+00$

$1,000 \mathrm{E}+00: 1.000 \mathrm{E}+00$

5.000E-01?5.000E-01

$3-1$

$-1$

$3-1$
$3-1$

$3-1$

$6.800 \mathrm{E}+01+6.800 \mathrm{E}+01$

$5.500 \mathrm{E}+0135.500 \mathrm{E}+01$

$5.000 \mathrm{E}+01^{3} 5.000 \mathrm{E}+01$

$1.600 E+02 ; 1.600 E+02$

5.000E-0i:5.000E-01
RAD SHAPE( 1)

RAD SHAPE( 2)

RAD SHAPE( 3 )

RAD SHAPE ( 4)

RAD_SHAPE( 5 )

RAD SHAPE ( 6)

RAD SHAPE 7

RAD SHAPE 8 )

RAD_SHAPE( 9)

RAD SHAPE (10)

RAD SHAPE (11)

RAD SHAPE (12)

FRACA (1)

FRACA( 2)

FRACA( 3)

FRACA (4)

FRACAC 5)

FRACA( 6)

FRACA 7 )

FRACAC 8)

FRACAC 93

FRACA (10)

FRACA(11)

FRACA(12)

DIET(1)

DIEr(2)

DIET(3)

DIET(4)

OIET(5)

OLET( 6$)$

SOIL

DWI

FOW

FHHW

FLW

3 FIRW

I FRQ

$0.500 E+00$ FPLANT

$0.100 E+01$ FMEAT

$0.100 \mathrm{E}+01$; FMLLK

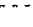

$\cdots$

$\cdots$
LFI5

LFI6

LW15

LWI6
LSI 
CVP-2005-00006

Rev. 0

IRESRAD, Version $6.22 \quad$ T« Limit $=0.5$ year $04 / 05 / 2005 \quad 12: 39$ Page 6

Summary: $100-k-55$ Shallow Zone Clean
File : $100-k-55$ Shallow Zone. RAD

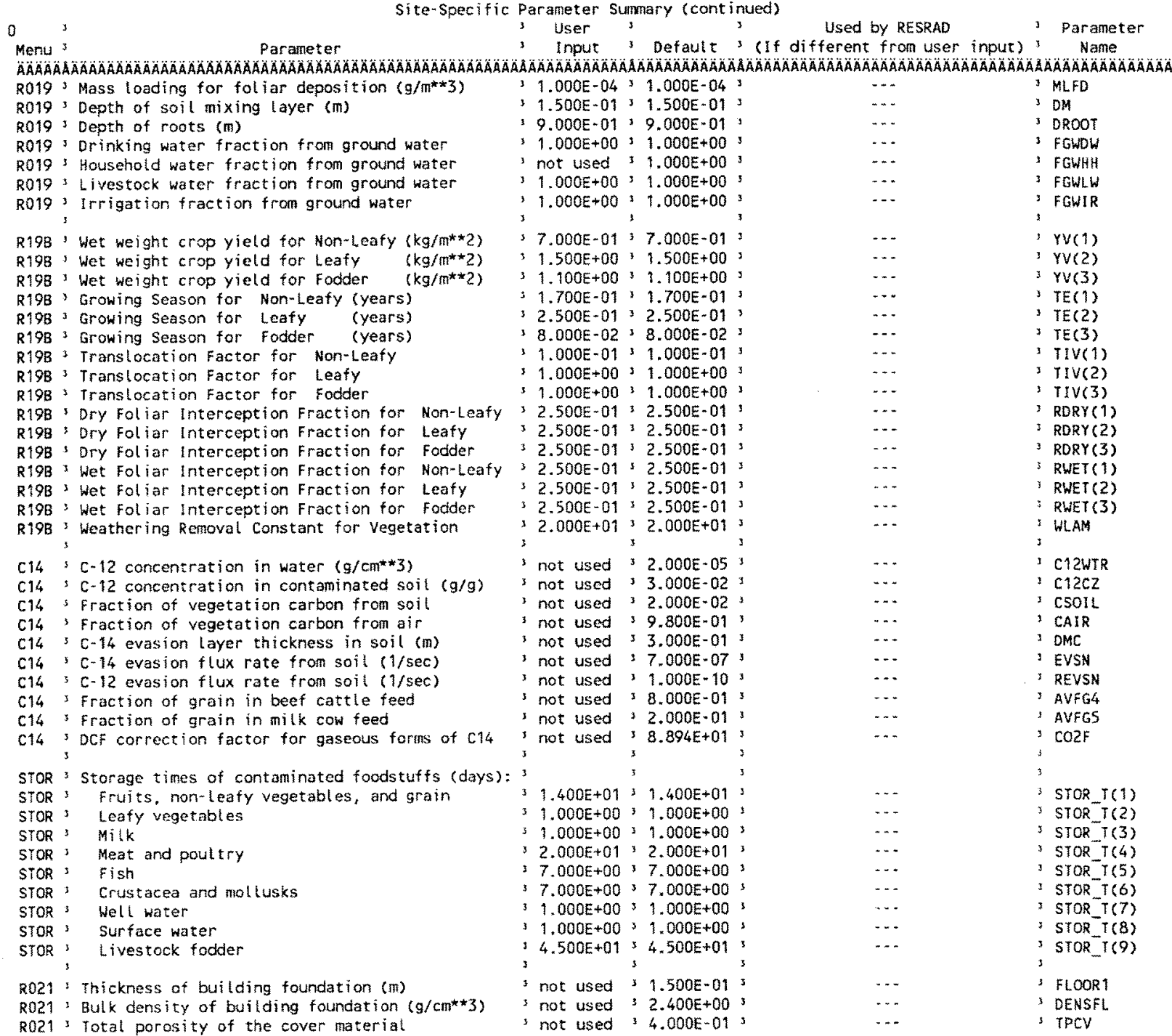


IRESRAD, Version $6.22 \quad T \ll$ Limit $=0.5$ year

$04 / 05 / 2005 \quad 12: 39$ Page 7

Sumary : $100-k-55$ shal low Zone cleanup verification

file: 100-K-55 shallow_zone. RAD

Site-specific Parameter Sumnary (continued)

$0_{\text {MenU }}^{3}$

Parameter

5 User 3 Used by RESRAD

Used by RESRAD 's parameter

R021 ' Volumetric water content of the cover materiat ; not used $35.000 E-02$;

R021 Diffusion coefficient for radon $g a s(\mathrm{~m} / \mathrm{sec})$

R021 3 in cover material

RO21 3 in foundation materiat

R021, in contaminated zone soil

R021' Radion vertical dimension of mixing (m)

R021 1 Average butlding air exchange rate (1/hr

R021 Height of the building (room) (m)

R021 Building interior area factor

R021 Buitding depth below ground surface (m)

R021 * Emanating power of Rn-222 gas

$\ldots$

PHZOFL

3

not used, 2,000E-06'

3 not used $33.000 E-07$ ?

3 not used $32.000 E-06$

3 not used $\$ 2.000 E+00$,

3 not used'5.000E-01'

3 not used $\$ 2.500 \mathrm{E}+00^{3}$

not used $\$ 0.000 E+00$ '

3 not used $\$-1.000 E+00$;

' not used $2.500 \mathrm{E}-01$ '

021 'Emanating power of Rn-220 gas

IIIL 3 Number of graphical time points

IITL 3 Maximum number of integration points for dose

IITL' Maximum number of integration points for risk

not used $31.500 \mathrm{E}-013$

3

$\begin{array}{ccc}3 & & 3 \\ s & \cdots & 3 \\ 3 & \cdots & 3\end{array}$

$\cdots$

3 P

Summary of Pathway selections

Pathway $s$ User Selection

AAAAA AAAAGAAAAAAAAAAAAAAAAAAAAAAAAAAAAAAAAAAAAAAAAAAAA

1 -. external gamna active

2 - inhatation ( $w / 0$ radon $)^{*}$ active

3 .. plant ingestion

4 -. meat ingestion $"$ active

5 - milk ingestion sactive

6 -. aquatic foods active

7 -. drinking water s active

8 - soil ingestion s active

9 -. radon " suppressed

Find peak pathway doses $y$ active

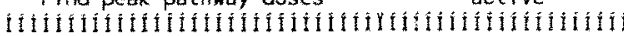


CVP-2005-00006

Rev. 0

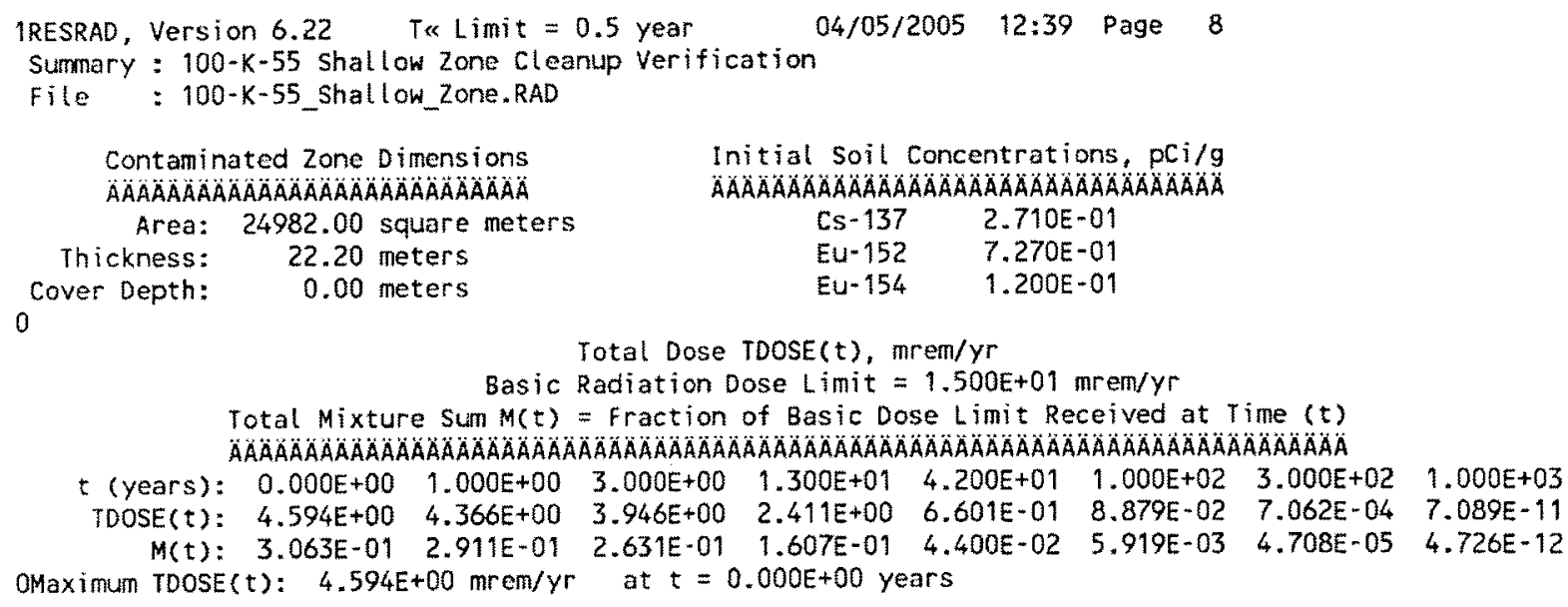


CVP-2005-00006

Rev. 0

RESRAD INPUT PARAMETERS FOR THE 100-K-55:1 DEEP ZONE 
CVP-2005-00006

Rev. 0

C-12 
CVP-2005-00006

Rev. 0

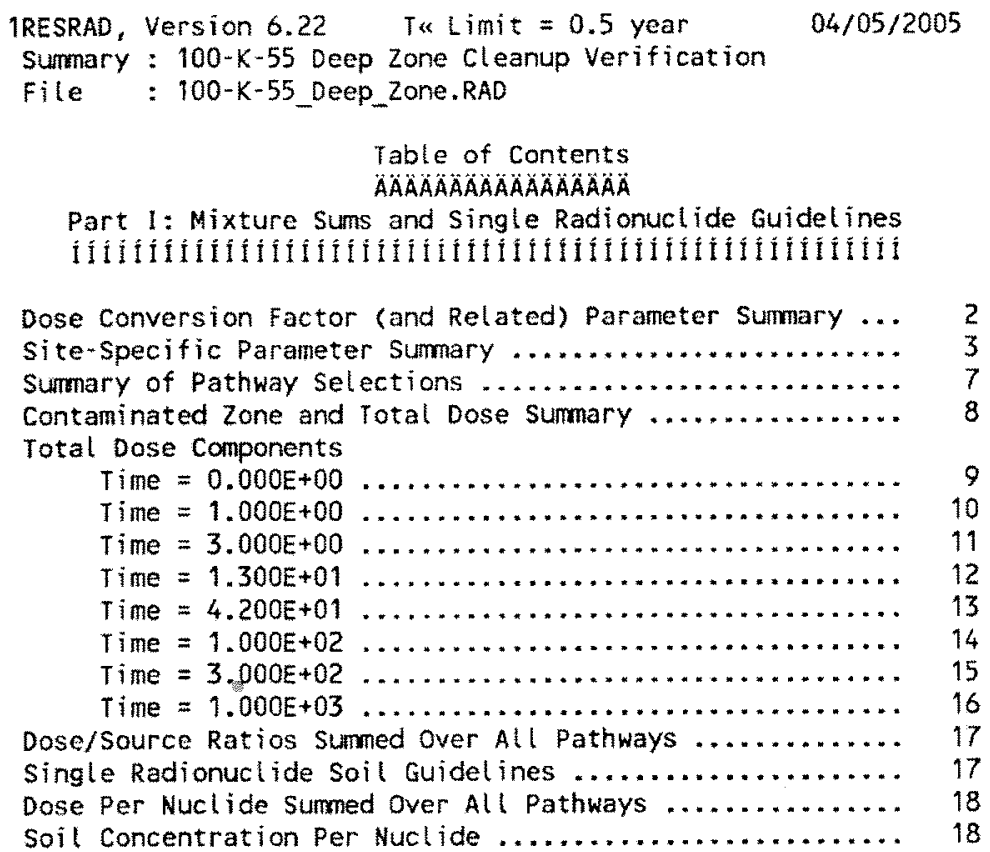


CVP-2005-00006

Rev. 0

\begin{tabular}{|c|c|c|c|c|c|c|c|c|}
\hline \multicolumn{9}{|c|}{$\begin{array}{c}\text { Dose Conversion Factor (and Related) Parameter Sumary } \\
\text { File: HEAST } 2001 \text { Morbidity }\end{array}$} \\
\hline & 3 & & & ' Current & 3 & & 3 & Parameter \\
\hline Menu & 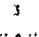 & & Parameter & Value & 3 & Default & 3 & Name \\
\hline$A A A A$ & & AAAAAAAAAA & AAAAAAAAAAAAAAAAAAAAAAAAAAAAAAAAAAAAAAAAAAAAAAA & AAAAAAAAAAAAAAAAA & & AAAAAAAAAAA & & \\
\hline B-1 & s & Dose conv & version factors for inhalation, inrem/pci: & 3 & 3 & & 3 & \\
\hline$B-1$ & 3 & $\operatorname{cs}-137+0$ & & $3.190 \mathrm{E}-05$ & 3 & $3.190 \mathrm{E}-05$ & 3 & $\operatorname{DCF} 2(1)$ \\
\hline$B-1$ & 3 & $E u-152$ & & $2.210 E-04$ & ? & $2.210 \mathrm{E}-04$ & 3 & $\operatorname{DCF} 2(2)$ \\
\hline$B-1$ & 3 & Eu-154 & & $2.860 E-04$ & 3 & $2.860 E-04$ & 3 & $\operatorname{DCF} 2(4)$ \\
\hline$B-1$ & $s$ & $6 d-152$ & & $2.430 E-01$ & 3 & $2.430 E-01$ & 3 & DCF2( 5) \\
\hline & 3 & & & s & 3 & & s & \\
\hline$D-1$ & 3 & Dose conv & ersion factors for ingestion, mrem/pCi: & 5 OOONE-05 & 3 & & 3 & \\
\hline$D=1$ & 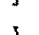 & $\mathrm{CS}-15(+1)$ & & $3.0001-02$ & ; & $\begin{array}{l}3.000 E-03 \\
6.480 F-06\end{array}$ & 3 & OCF $3(1)$ \\
\hline-1 & 3 & $E U-152$ & & $3.480 \mathrm{E}-00$ & $x^{2}$ & $0.480 E-00$ & 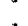 & DCtS 2$)$ \\
\hline-1 & 3 & $E U-154$ & & $9.550 E-06$ & 3 & $9.550 E-06$ & 3 & $\operatorname{DCF} 3(4)$ \\
\hline$D-1$ & 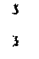 & $6 d-152$ & & $1.610 E-04$ & 3 & $1.610 E-04$ & 3 & $\operatorname{DCF} 3(5)$ \\
\hline$D-34$ & $x^{3}$ & Food tran & isfer factors: & y & 3 & & 3 & \\
\hline$D-34$ & i & $C s-137+D$ & - plant/soil concentration ratio, dimensionless & $34.000 E-02$ & 3 & 4. DOOE -02 & 3 & $\operatorname{RTF}(1,1)$ \\
\hline$D-34$ & 3 & $\operatorname{cs}-137+D$ & , beef/livestock-intake ratio, $(p C i / k g) /(p \mathrm{Ci} / \mathrm{d})$ & $3.000 E-02$ & 3 & $3.000 E-02$ & 3 & $\operatorname{RTF}(1,2)$ \\
\hline$D-34$ & 3 & $\mathrm{Cs}-137+\mathrm{D}$ & , milk/livestock-intake ratio, $(p c i / L) /(p c i / d)$ & $8.000 E-03$ & 3 & $8.000 E-03$ & 3 & $\operatorname{RTF}(1,3)$ \\
\hline$D-34$ & 3 & & & 3 & 3 & & $\mathrm{~s}$ & \\
\hline$D-34$ & 3 & $E u-152$ & - plant/soil concentration ratio, dimensionless & $2.500 E-03$ & 3 & $2.500 E+03$ & 3 & $\operatorname{RTF}(2,1)$ \\
\hline$D-34$ & 3 & Eu-152 & , beef/livestock-intake ratio, $(\mathrm{pCi} / \mathrm{kg}) /(\mathrm{pCi} / \mathrm{d})$ & $2.000 \mathrm{E}-03$ & 3 & $2.000 E-03$ & 3 & $\operatorname{RTF}(2,2)$ \\
\hline $\begin{array}{l}D-34 \\
D-34\end{array}$ & 3 & Eu-152 & milk/livestock-intake ratio, $(\mathrm{pci} / \mathrm{l}) /(\mathrm{pCi} / \mathrm{d})$ & $2.000 E-05$ & 3 & $2.000 E-05$ & 3 & $\operatorname{RTF}(2,3)$ \\
\hline$D-34$ & 3 & Eu-154 & - plant/soil concentration ratio, dimensiontess & $2.500 E-03$ & 3 & $2.500 E-03$ & 3 & $\operatorname{RTF}(4,1)$ \\
\hline$D-34$ & 3 & Eu-154 & , beef/livestock-intake ratio, $(\mathrm{pCi} / \mathrm{kg}) /(\mathrm{pCi} / \mathrm{d})$ & $2.000 E-03$ & 3 & $2.000 E-03$ & 3 & $\operatorname{RTF}(4,2)$ \\
\hline$D-34$ & 3 & $E u-154$ & milk/livestock-intake ratio, $(\mathrm{pCi} / \mathrm{L}) /(\mathrm{pCi} / \mathrm{d})$ & $2.000 E-05$ & & $2.000 E-05$ & 3 & $\operatorname{RTF}(4,3)$ \\
\hline$D-34$ & 3 & & & 3 & 3 & & 3 & \\
\hline$D-34$ & 3 & $60-152$ & - plant/soil concentration ratio, dimensionless & $2.500 E-03$ & 3 & $2.500 E-03$ & 3 & $\operatorname{RTF}(5,1)$ \\
\hline$D-34$ & $s$ & $6 d-152$ & - beef/livestock-intake ratio, $(\mathrm{pci} / \mathrm{kg}) /(\mathrm{pCi} / \mathrm{d})$ & $2.000 E-03$ & 3 & 2.000E $=03$ & 3 & $\operatorname{RTF}(5,2)$ \\
\hline$D-34$ & 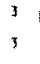 & $6 d-152$ & milk/livestock-intake ratio, $(\mathrm{pCi} / \mathrm{L}) /(\mathrm{pCi} / \mathrm{d})$ & $2.000 E-05$ & & $2.000 E-05$ & 3 & $\operatorname{RTF}(5,3)$ \\
\hline$D-5$ & 3 & Bioaccunu & dation factors, fresh water, l/kg: & $s$ & $s$ & & 3 & \\
\hline $0-5$ & \pm & $C s-137+0$ & , fish & $2.000 E+03$ & 3 & $2.000 E+03$ & $\mathrm{~s}$ & BIOFAC $(1,1)$ \\
\hline D-5 & 3 & $\mathrm{Cs}-137+\mathrm{D}$ & , crustacea and mollusks & $1.000 E+02$ & & $1.000 E+02$ & 3 & BIOFAC $(1,2)$ \\
\hline$D \cdot 5$ & 3 & & & $s$ & 3 & & 3 & \\
\hline$D-5$ & $s$ & Eu- 152 & f fish & $5.000 E+01$ & 3 & $5.000 E+01$ & 3 & BIOFAC $(2,1)$ \\
\hline D-5 & 3 & $E u-152$ & , crustacea and mollusks & $1.000 E+03$ & & $1.000 \mathrm{E}+03$ & 3 & $\operatorname{BIOFAC}(2,2)$ \\
\hline$D-5$ & $s$ & & & 3 & 3 & & 3 & \\
\hline$D-5$ & 3 & Eu-154 & , fish & $5.000 E+01$ & 3 & $5.000 E+01$ & 3 & BIOFAC $(4,1)$ \\
\hline D-5 & 3 & $E u-154$ & , crustacea and mollusks & $1.000 E+03$ & & $1.000 E+03$ & 3 & $\mathrm{~B} 10 \mathrm{FAC}(4,2)$ \\
\hline$D-5$ & 3 & & & 3 & 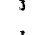 & & 3 & \\
\hline & 3 & $6 d-152$ & - fish & $2.500 E+01$ & 3 & $2.500 E+07$ & 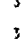 & $\begin{array}{l}\text { BIOFAC }(5,1) \\
\text { BIOFAC }(5,2)\end{array}$ \\
\hline & & & crustacea and mollusks & 1.000E+03 & & $1.000 t+05$ & & BIOFALC 5 , \\
\hline
\end{tabular}


CVP-2005-00006

Rev. 0

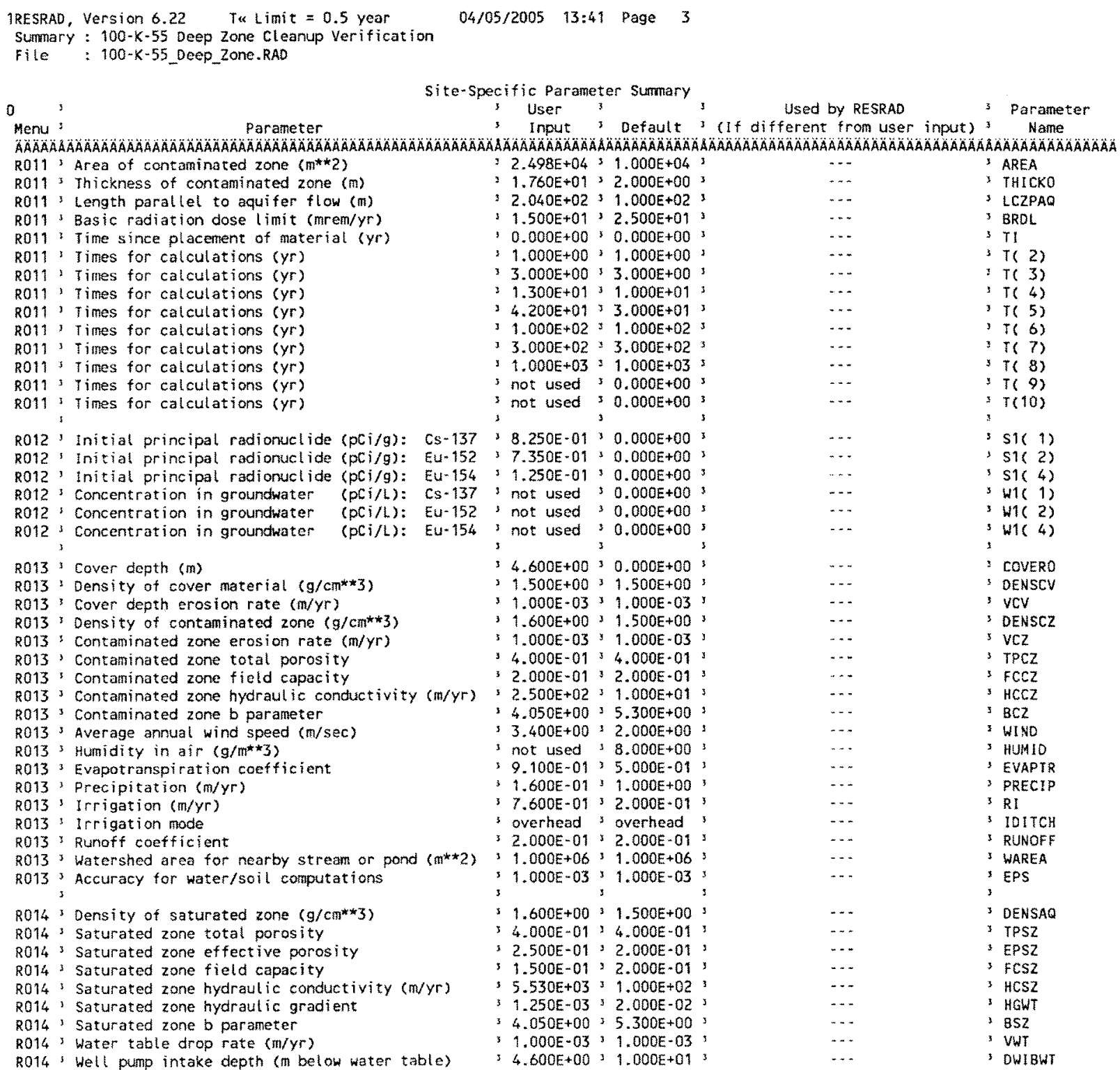


CVP-2005-00006

Rev. 0

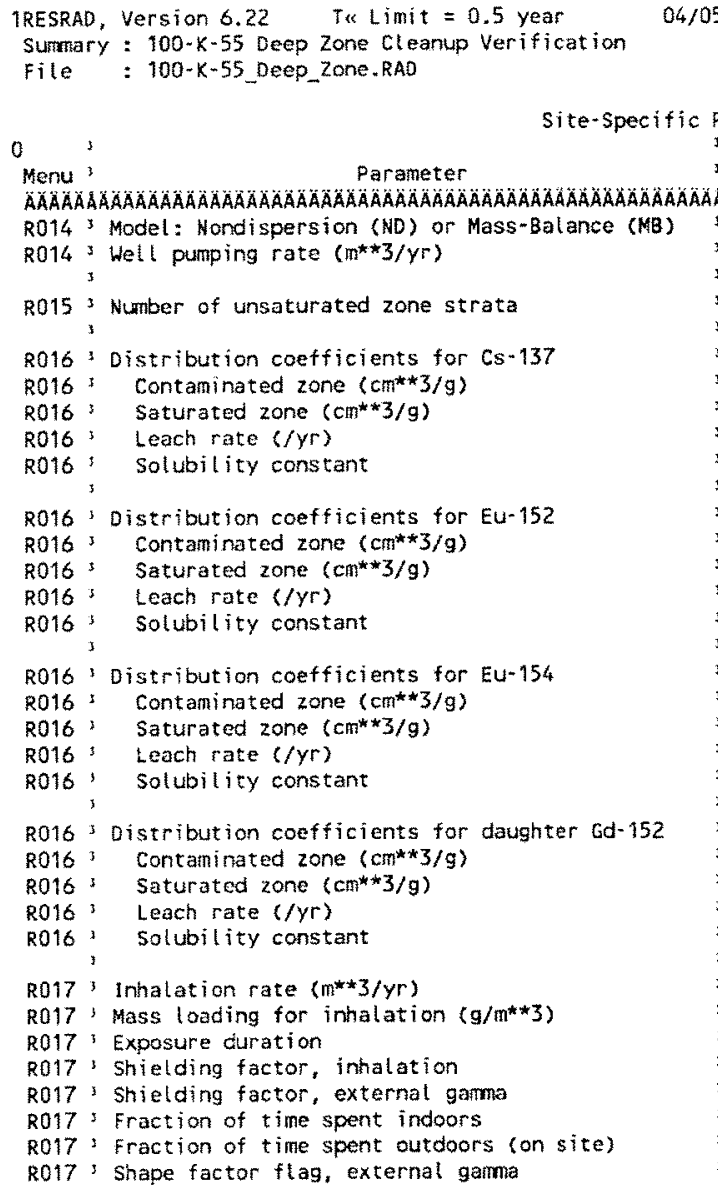

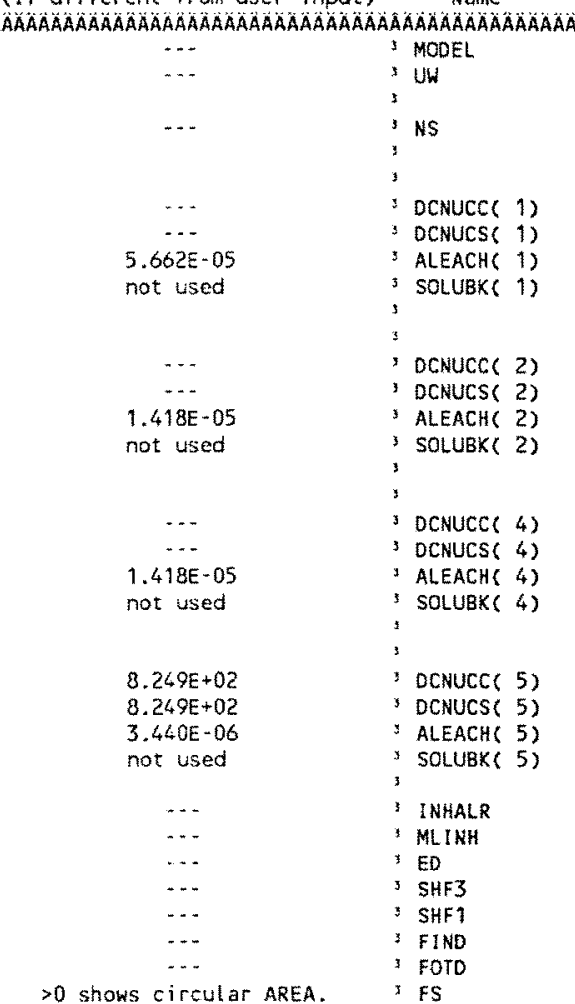


CVP-2005-00006

Rev. 0

1RESRAD, version 6.22 Ta Limit $=0.5$ year

04/05/2005 13:41 Page 5

Sumary : $100-k-55$ Deep zone cleanup Verification

file: $100-K-55$ Deep Zone. RAD

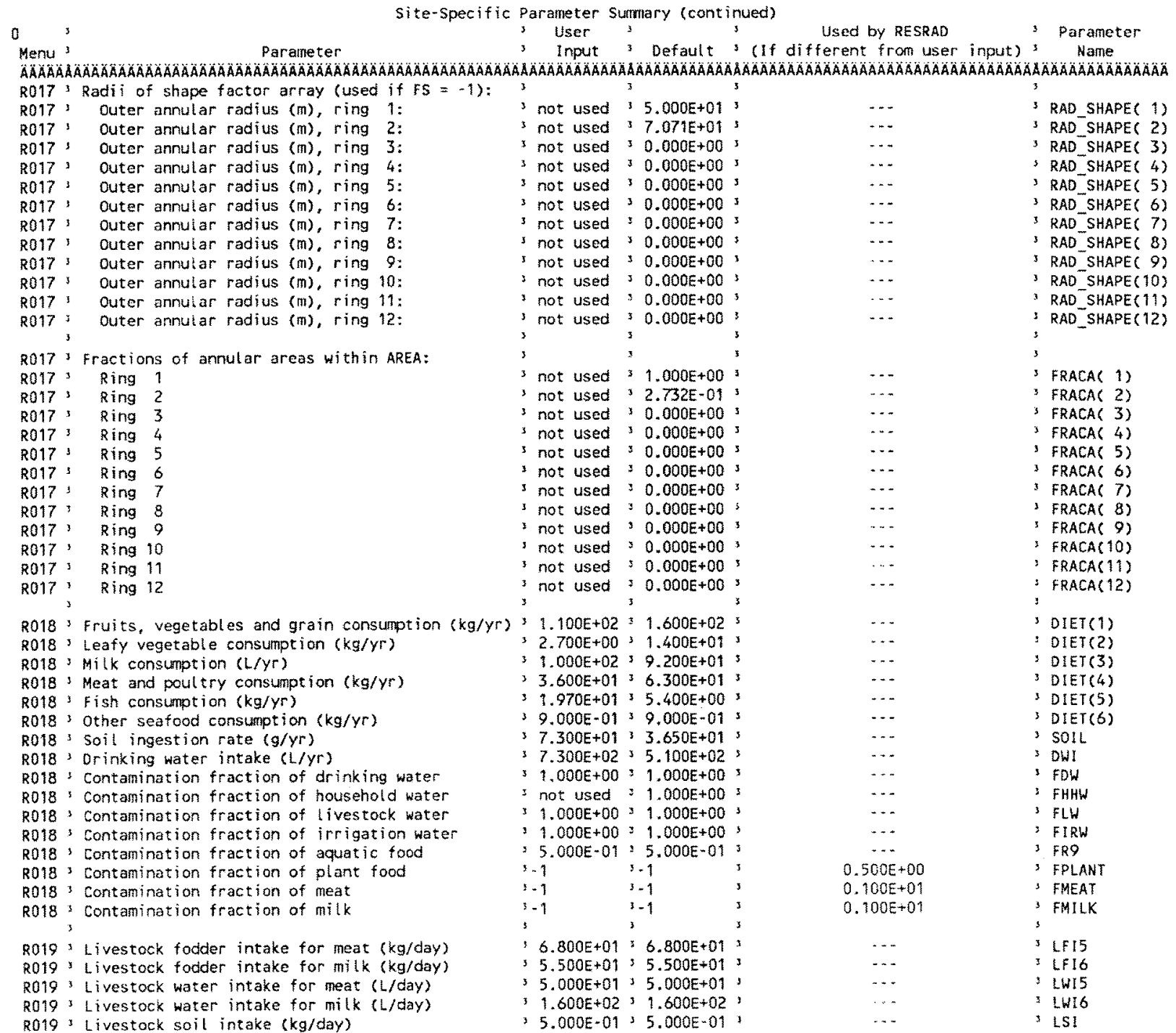


CVP-2005-00006

Rev. 0

IRESRAD, Version 6.22 Te Limit $=0.5$ year

04/05/2005 13:41 Page 6

Stmmary : 100-K-55 Deep Zone Cleanup verification

file: $100-K-55$ Deep_zone.RAD

0

Site-Specific Paraneter Sumary (continued)

Menus

Parameter

, User,

Used by RESRAD

3 Parameter

In

R019 ' Mass loading for fol lar depos tion $\left(\mathrm{g} / \mathrm{m}^{* *}\right.$

R019' Depth of soit mixing layer (m)

I $1.000 \mathrm{E}-04: 1.000 \mathrm{E}=04$

$3.500 \mathrm{E}-01: 1.500 \mathrm{E}-01$

$9.000 E-01: 9.000 E-01$

$: \begin{aligned} & 9.000 E-01: 9.000 E+01 \\ & 1.000 E+00: 1.000 E+00\end{aligned}$

not used $31.000 E+00$

$1.000 E+00 \div 1,000 E+00$

$1.000 \mathrm{E}+00 ; 1.000 \mathrm{E}+00$

R019: Household water fraction from ground water

RO19 S Livestock water fraction from ground water

;

$R 19 B$ s Wet weight crop yield for Non-Leafy $\left(\mathrm{kg} / \mathrm{m}^{* *} \mathrm{2}\right)$

$7.000 \mathrm{E}-01 ; 7.000 \mathrm{E}-01$

R19B Wet weight crop yield for leafy $\left(\mathrm{kg} / \mathrm{m}^{* *}\right)$

$1.500 E+00+1.500 E+00$

R19B ' Wet wight crop yield for Fodder ( $\left.\mathrm{kg} / \mathrm{m}^{*}{ }^{*} 2\right)$

R198 Growing Season for Non-Leafy (years)

$R 19 B$ S Growing Season for leafy (years)

$1.100 E+00 \div 1.100 E+00$

, $1.700 E-01 ; 1.700 E-01$

$2.500 E-01 \cdot 2.500 E-01$

$8.000 E-02 \times 8.000 E-02$

$1.000 E-01 \times 1.000 E-01$

R198 3 Iranslocation Factor for Non-Leafy

$1.000 E+00: 1.000 E+00$

R198 3 Iranslocation Factor for Leafy

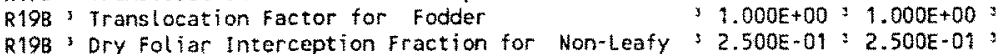

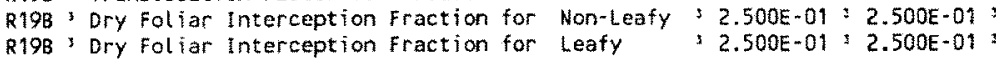

198 3 Dry Foliar Interception Fraction for Fodder $: 2.500 E-01: 2.500 E-01$

R19B: Wet Foliar Interception Fraction for Non-Leafy $32.500 E-0132.500 \mathrm{E}-01$

R19B ${ }^{3}$ Wet foliar interception Fraction for Leafy, $2.500 \mathrm{E}-0132.500 \mathrm{E}-01$

R19B Wet Foliar interception fraction for Fodder $32.500 E-01 ; 2.500$-01

R19B Weathering Removal Constant for Vegetation

$\begin{array}{lll}2.500 E-01 & 2.500 E-01 \\ 2.000 E+01 & 3.000 E+01\end{array}$

not used $: 2,000 \mathrm{E}-05$

not used 3 3.000E-02

not used $2.000 \mathrm{E}-02$

not used $: 9.800 \mathrm{E}-0 \mathrm{i}$

"not used 3 3.000E-01

inot used $? 7.000 \mathrm{O}-07$

not used 1 , $000 E-10$,

"not used $8.000 \mathrm{E}-01$

not used $2.000 \mathrm{E}-01$

not used; $8.894 E+01$ :

C14, Fraction of grain in milk cow feed

$1.400 E+01 ; 1.400 E+01$

$31.000 E+0031.000 E+00$

$31.000 E+00 \div 1.000 E+00$

, $2.000 E+0132.000 E+01$.

, $7.000 E+00 ; 7.000 E+00$

$7.000 E+00 ; 7.000 E+00^{3}$

$31.000 \mathrm{E}+00: 1.000 \mathrm{E}+00$

$11.000 E+00 \div 1.000 E+00$

$\times 4.500 E+01 ; 4.500 \mathrm{E}+01$

Surface water

STOR: Livestock fodder

R021 Thickness of building foundation ( $m$ )

3 not used $31.500 E-01$

3 not used $: 2.400 E+00$

3 not used 3 4.000E-01'

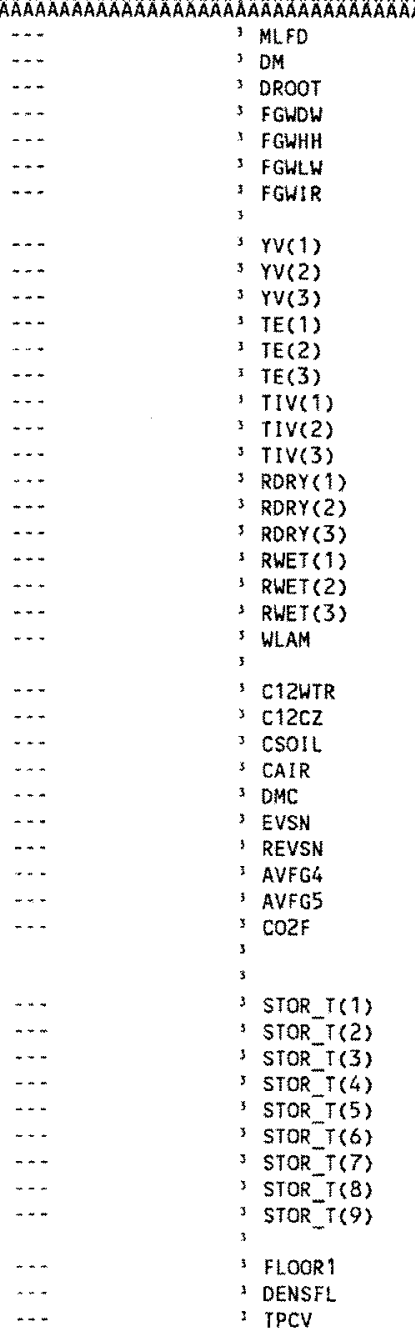


CVP-2005-00006

Rev. 0

1RESRAD, Version $6.22 \quad$ T* Limit $=0.5$ year

$04 / 05 / 2005 \quad 13: 41$ Page 7

Sumary : $100-\mathrm{K}-55$ Deep zone cleanup Verification

file : 100-K-55 Deep_Zone.RAD

\begin{tabular}{|c|c|c|c|c|c|c|c|c|c|}
\hline \multicolumn{10}{|c|}{ Site-Specific Parameter Sumary (continued) } \\
\hline 0 & 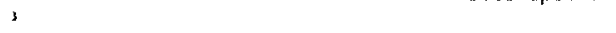 & US & ser & 3 & & & Used bY RESRAD & s & Parameter \\
\hline Menu & Parameter & $=$ In & nout & 3 & Default & (If & different from user input) & & Name. \\
\hline AAAAÁ & 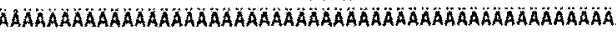 & & $\triangle A A A A A C$ & & & & AAAAAAAAAAAOAAAAAAAAAAAAAAAA & & AAAAAAAAAAA \\
\hline R021 & Total porosity of the building foundation & not & used & 3 & $1.000 E-01$ & 3 & ... & $s$ & TPFL \\
\hline Ro21 & - volumetric water content of the cover material & not & used & ${ }^{3}$ & $5.000 E-02$ & s & $\cdots$ & , & PH2OCV \\
\hline RO21 & 3 Volunetric water content of the foundation & not & used & 3 & $3.000 \mathrm{E}-02$ & 3 & -- & 3 & PH2OFL \\
\hline RO21 & 'Diffusion coefficient for radon gas (m/sec): & 3 & & 3 & & 3 & & s & \\
\hline R021 & 3 in cover material & s not & used & 3 & $2.000 \mathrm{E}-06$ & 3 & $\cdots$ & 3 & DIfCV \\
\hline R021 & in foundation material & not & used & 3 & $3.000 \mathrm{E}-07$ & 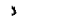 & $\cdots$ & 3 & DIFFL \\
\hline RO21 & in contaminated zone soil & not & used & 3 & $2.000 E-06$ & 3 & $\cdots$ & 3 & DIFCZ \\
\hline R021 & Radon vertical dimension of mixing (m) & 3 not & used & 3 & $2.000 \mathrm{E}+00$ & : & $\cdots$ & 3 & HMIX \\
\hline R021 & 3 Average building air exchange rate (1/hr) & not & used & s & $5.000 E-01$ & 3 & +. & s & REXG \\
\hline R021 & 3 Height of the building (room) (n) & s not & used & 3 & $2.500 E+00$ & 3 & $\cdots$ & 3 & HRM \\
\hline $\mathrm{R} 021$ & Building interior area factor & 3 not & used & 3 & $0.000 E+00$ & 3 & $\cdots$ & 3 & FAI \\
\hline R021 & \&uilding depth below ground surface (m) & $\therefore$ not & used & 3. & $-1.000 E+00$ & s & $\ldots$ & $\mathfrak{3}$ & DMFL \\
\hline R021 & - Enanating power of $\mathrm{Rn}^{-222}$ gas & , not & used & 3 & $2.500 E-01$ & 3 & $\cdots$ & 3 & EMANA( 1$)$ \\
\hline R021 & 1 Emanating power of $R n-220$ gas & $\therefore$ not & used & 3 & $1.500 E-01$ & 3 & $\cdots$ & $x$ & EMANA(2) \\
\hline & 3 the the & , & & : & & 3 & & 3 & \\
\hline TITL & Number of graphical time points & 3 & 32 & 3 & $\ldots$ & ? & $\cdots$ & $:$ & NPTS \\
\hline TITL & 3 Maximum number of integration points for dose & 3 & 1 & 3 & $\ldots$ & : & ... & s & LYMAX \\
\hline TITL & I Maximum number of integration points for risk & $s$ & 5 & 3 & $\ldots$ & 3 & ... & & KYMAX \\
\hline
\end{tabular}

Sumary of Pathway Selections

Pathway 3 User selection

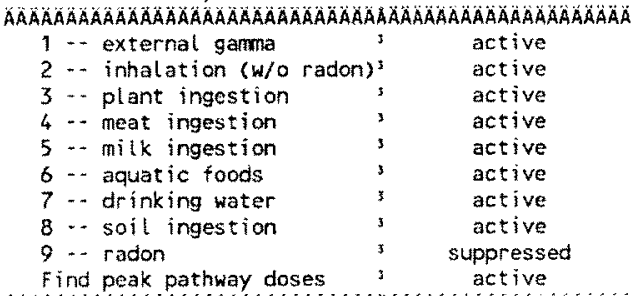

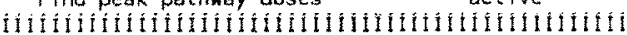


CVP-2005-00006

Rev. 0

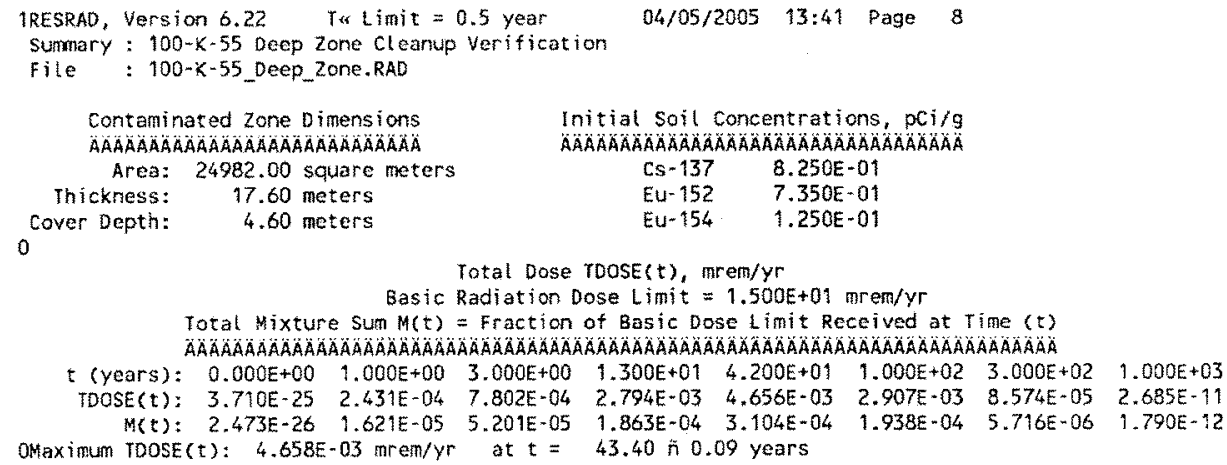


CVP-2005-00006

Rev. 0

RESRAD INPUT PARAMETERS FOR THE

100-K-55:1 AND 100-K-56:1 COMBINED OVERBURDEN 
CVP-2005-00006

Rev. 0

C-22 
CVP-2005-00006

Rev. 0

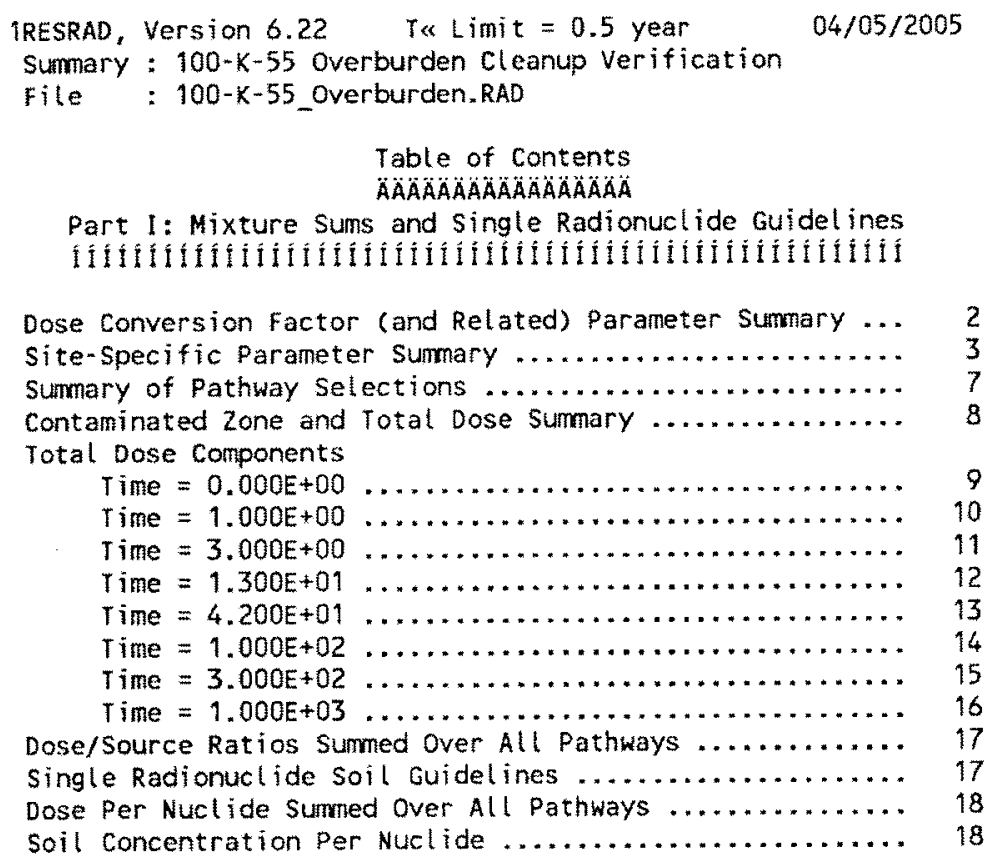


CVP-2005-00006

Rev. 0

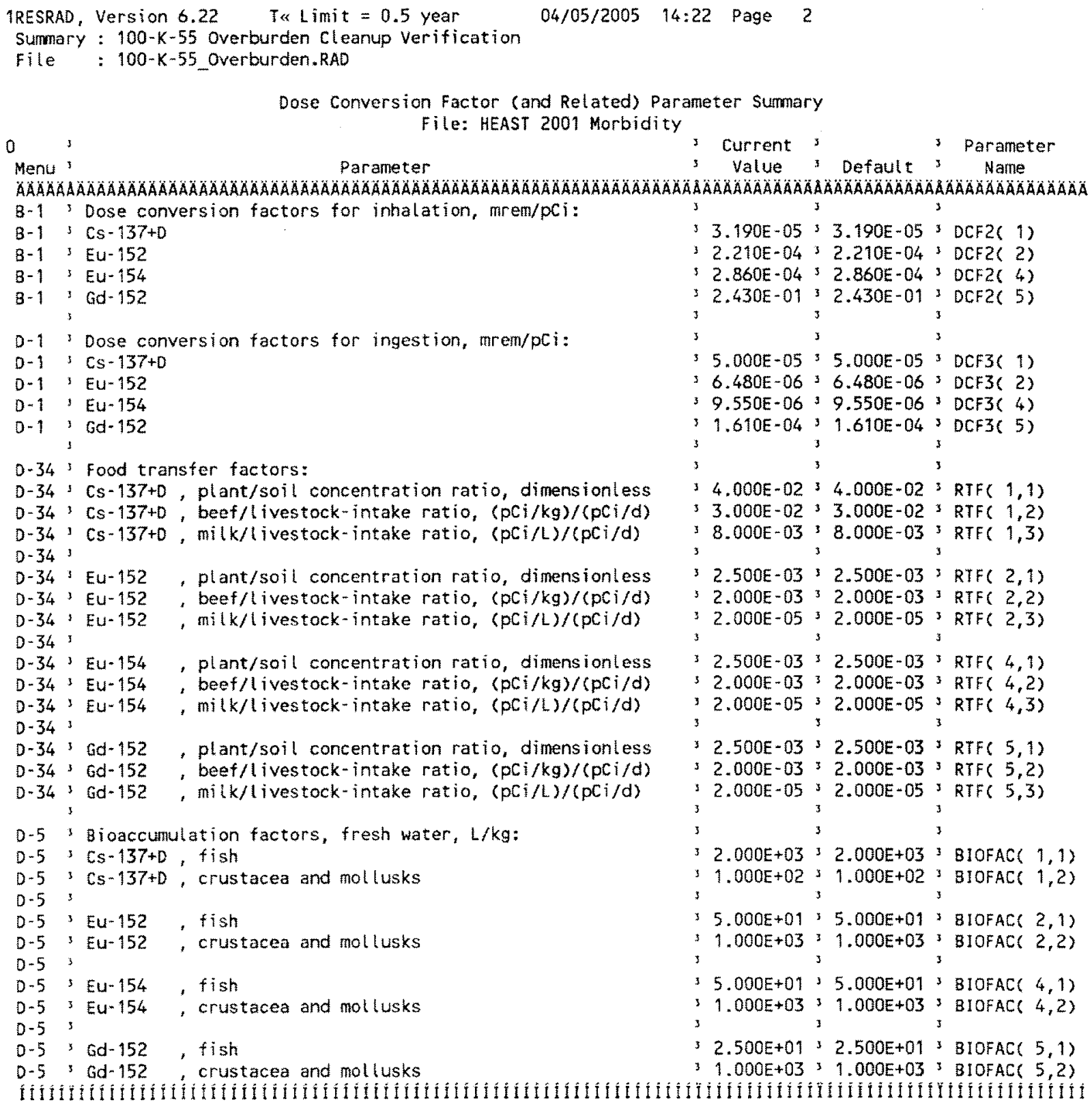


1RESRAD, Version 6.22 T 4 Limit $=0.5$ year

$04 / 05 / 2005 \quad 14: 22$ Page 3

Sumary : $100-X-55$ overburden cleanup verification

File: $100-K-55$ overburden. RAD

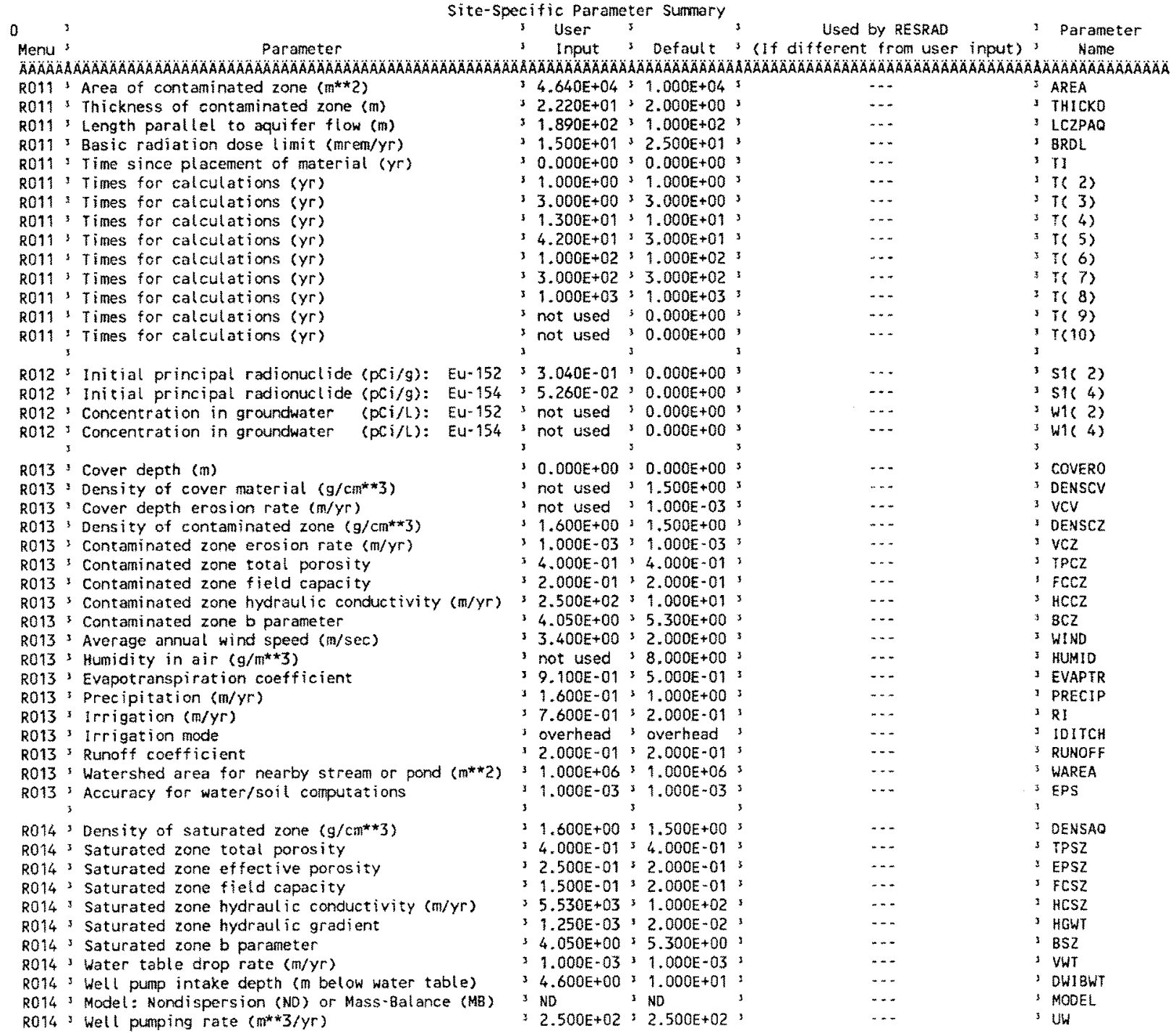




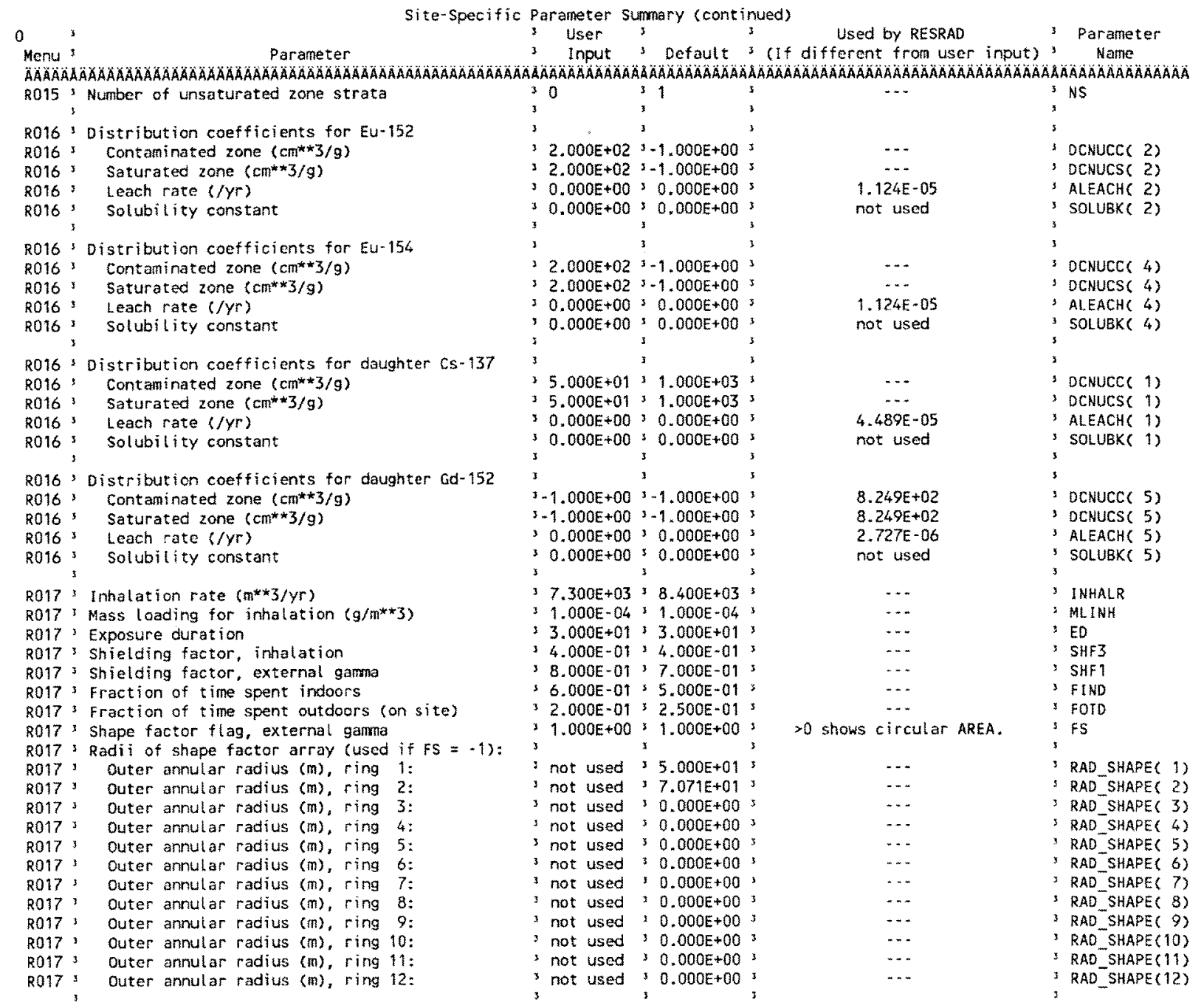


CVP-2005-00006

Rev. 0

IRESRAD, Version 6.22 T\& Limit $=0.5$ year

04/05/2005 14:22 Page 5

Sumary : $100-k-55$ overburden Cleanup verification

file: $100-K-55$ _overburden.RAD

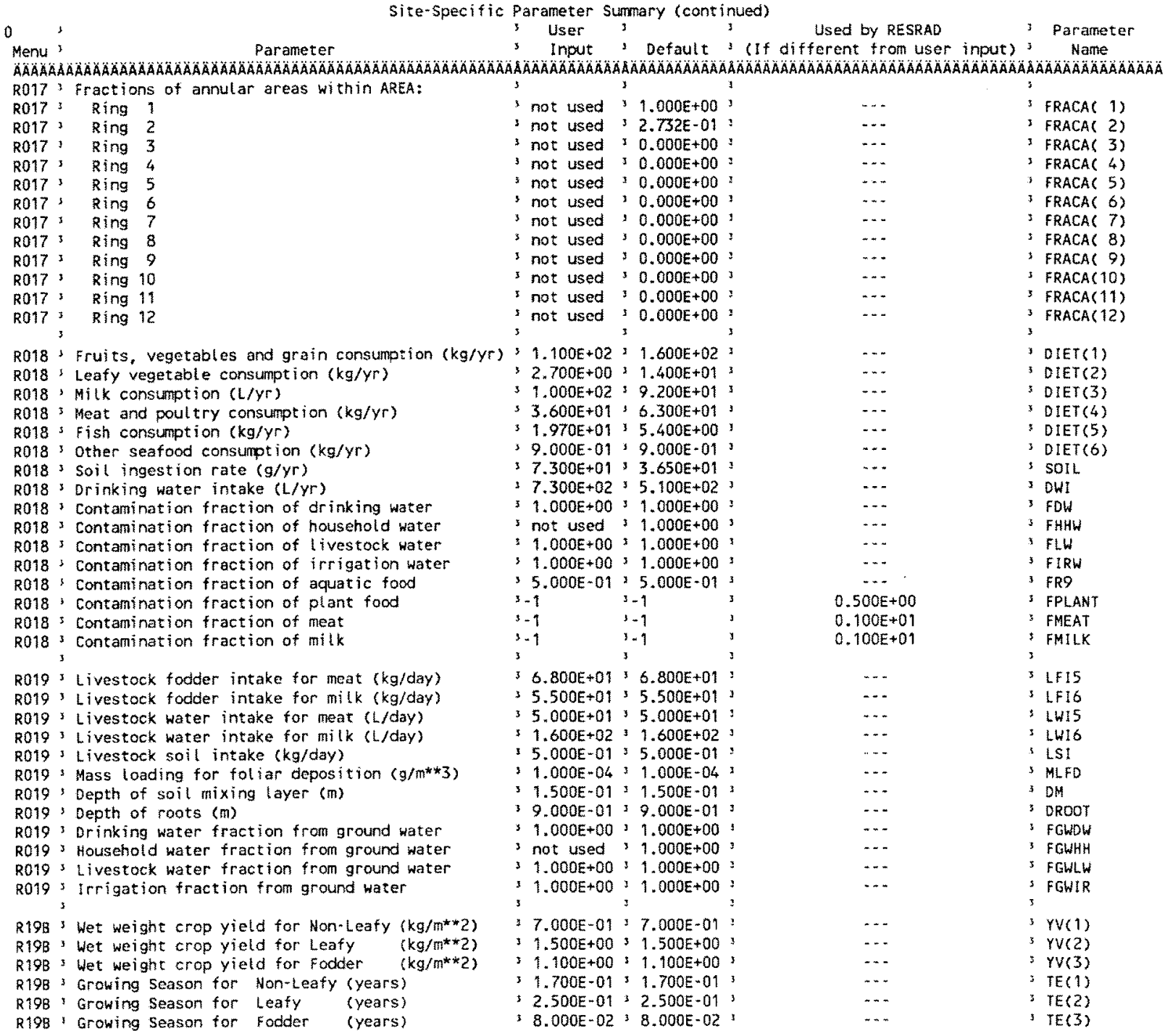


CVP-2005-00006

Rev. 0

IRESRAD, Version 6.22 Ta Limit $=0.5$ year

04/05/2005 14:22 Page 6

Sumary : $100-k-55$ overburden cleanup verification

File: 100-k-55_overburden.RAD

Site-Specific Parameter Sumary (cont inued)

0

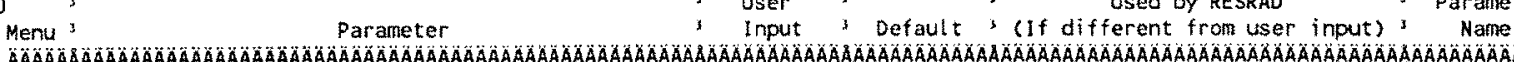

User, Used by RESRAD

3 Parameter

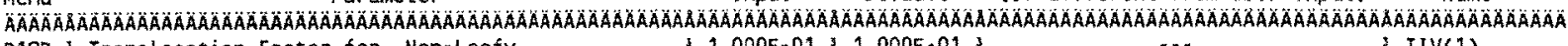
R198 Translocation Factor for Non-Leafy $\quad 31.000 E=01^{3} 1.000 E-01^{3} \quad$ IIV(1)

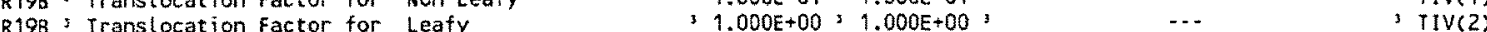

R198 Transtocation Factor for Fodder

R198, Dry Foliar Interception Fraction for Non-Leafy $2.500 E-01$; $2.500 E-01$ '

R198 : Dry Foliar Interception Fraction for leafy, $2.500 \mathrm{E}-01,2.500 \mathrm{E}-01$

R198 ' Dry Foliar Interception Fraction for Fodder $32.500 E-01 ; 2.500 E-01$

R193 3 Wet Foliar Interception Fraction for Non-Leafy; 2.500E-01;2.500E-01.

R19B T Wet follar interception Fraction for Leafy 32.500 - $0132.500 E-01$,

R19B : Wet Foliar Interception Fraction for Fodder $32.500 E-0132.500$ - 01

R19B : Weathering Removal Constant for Vegetation $; 2.000 E+01: 2.000 E+01$;

$0-12$ concentration in water $\left(g / \mathrm{cm}^{*} * 3\right)$

$\begin{array}{ll}C 14 ; & \mathrm{C}-12 \text { concentration in water }(\mathrm{g} / \mathrm{cm} * \mathrm{k}) \\ \mathrm{C} 14 & \mathrm{C}-12 \text { concentration in contaminated soil }(\mathrm{g} / \mathrm{g})\end{array}$

C14 3 Fraction of vegetation carbon from soll

C14. Fraction of vegetation carbon from air

C14. $\mathrm{C}-14$ evasion tayer thickness in soll (m)

C14 $+\mathrm{C}-14$ evasion flux rate from soil $(1 / \mathrm{sec})$

C14: $\mathrm{C}-12$ evasion flux rate from soil $(1 / \mathrm{sec})$

C14 : Fraction of grain in beef cattle feed

C14, Fraction of grain in milk cow feed

C14 'DCF correction factor for gaseous forms of $\mathrm{C14}$

STOR' Storage times of contaminated foodstuffs (days):

STOR ${ }^{3}$ Leafy vegetables

STOR 3 Milk

STOR ' Meat and poultry

STOR: Fish

STOR ; Crustacea and mollusks

STOR, Well water

SIOR \& Surface water

STOR 3 Livestock fodder

R021 Thickness of building foundation (m)

not used $2.000 E-05$

not used $3.000 E-02$

not used, $2.000 \mathrm{E}-02$

not used $99.800 \mathrm{E}-01$

not used $3.000 E-01$

inot used $\$ 7.000 E-07$

not used $31.000 E-10$

not used 8.000 - 01

not used $2.000 E-01$

' not used ' $8.894 \mathrm{E}+01$ '

$1.400 E+01 ; 1.400 E+01$

$1.000 E+00: 1.000 E+00$

$31.000 E+0031.000 E+00$

$32.000 E+01 ; 2.000 E+01$

$3.000 E+00: 7.000 E+00$

$7.000 E+00 ; 7.000 E+00$

$1.000 E+00 ; 1.000 E+00$

$1.000 E+00>1.000 E+00$

$3.500 \mathrm{E}+0134.500 \mathrm{E}+01$

not used, $1.500 E-01$

not used $2.400 E+00$

3 not used; 4.000E-01

3 not used $31.000 E-01:$

RO21: Total porosity of the cover materiat

R021: Total porosity of the bullding foundation

R021 : Volumetric water content of the cover material

R021 Volumetric water content of the foundation

R021 Diffusion coefficient for radon gas (m/sec):

Roz' 3 in cover material

ROZT' in foundation material

ROZ1' in contaminated zone soil

R021 3 Radon vertical dimension of mixing (m)

R021: Average building air exchange rate $(1 / \mathrm{hr})$

R021 ${ }^{3}$ Height of the building (room) (m)

R021 Building interior area factor

RO21 3 Building depth below ground surface (m)

not used 3.000 E-02

3 not used $32.000 \mathrm{E}-06$

3 not used 3 3.000E-07,

not used 2.000 E-06

3 not used $32.000 E+00$

3 not used; 5.000E-01

I not USed $32.500 E+00$

3 not used $\$ 0.000 E+00$

3 not used $1-1.000 E+00$

not used $\$ 2.500 E-01$

not used? $1.500 \mathrm{E}-01$ ?

$\ldots \quad$ it

-..

a RDRY(2)

I RORY (3)

+.. S RWET(1)

-.. RUET(Z)

... RWET(3)

- S WLAM

3 C12UTR

a. $3 \mathrm{Cl} C \mathrm{CZ}$

3 CSOIL

3 CAIR

DMC

$\ldots$-..

- 3 REVSN

3 AVFG4

A AVFG

CO2F

$\begin{array}{ll}\ldots & \text { s STOR_T(1) } \\ \ldots & \text { sTOR T(2) }\end{array}$

; STOR I(3)

J STORTT(4)

: STOR T(5)

- $\quad$ STORT T(O)

, STORT(T)

-. STTORT(8)

$\ldots \quad 3 \operatorname{STOR}^{-1} T(9)$

.. 3 FLOR

... DENSFL

-.

-.-

-. $\quad$ PH2OCV

-.. PHZOFL

$\cdots \quad 3$ DIFCV

$\cdots$

$\cdots$

… $\quad$ DIFCZ

... HMIX

$\cdots+$ REXC

-..

$\cdots$ FAI

...

...

R021: Emanating power of $R n-220$ gas

EMANA (2) 
CVP-2005-00006

Rev. 0

1RESRAD, Version $0.22 \quad$ T\& Limit $=0.5$ year

Surnary : $100-k-55$ overburden cleanup verification

File: 100-K-55 overburden.RAD

04/05/2005 14:22 Page 7

\begin{tabular}{|c|c|c|c|c|c|c|c|c|c|}
\hline \multicolumn{10}{|c|}{ Site-specific Paraneter Sumary (continued) } \\
\hline & $x^{-1}$ & , & User & 3 & & 3 & Used by RESRAD & $\mathrm{J}$ & Parameter \\
\hline Henu & Parameter & 3 & Input & , & Default & (If & different from user input) & $\$$ & Name \\
\hline$\triangle A A A A$ & 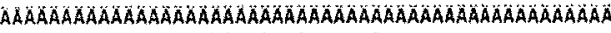 & & AAAAAAACA & & ÄAAAAAAAAAÁ & AAAAAAAA & AAAAAAAAAAOAAAOAAAAAAAAAAAAOAA & & $\triangle A A A A A M A A A A A A A$ \\
\hline TITL & Number of graphical time points & s & 32 & s & $\cdots$ & 3 & $\ldots$ & & NPTS \\
\hline TITL & "Maximum number of integration points for dose & ? & 1 & $y$ & $\cdots$ & 3 & $\cdots$ & & LYMAX \\
\hline $1 T L$ & ' Maximum number of integration points for risk & $:$ & 5 & 3 & ... & 3 & $\cdots$ & & KYMAX \\
\hline
\end{tabular}

Summary of Pathway Selections

Pathway 'User Selection

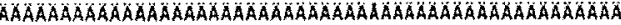

1 -- external gamma

2 - inhalation (w/o radon)' active

3 -. plant ingestion active

4 .. meat ingestion active

5 .. milk ingestion

6 aquatic foods active

7 .. drinking weter active

8 -. soit ingestion active

9 -- radon suppressed

find peak pathway doses, active

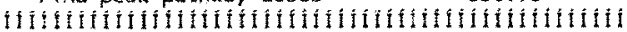


CVP-2005-00006

Rev. 0

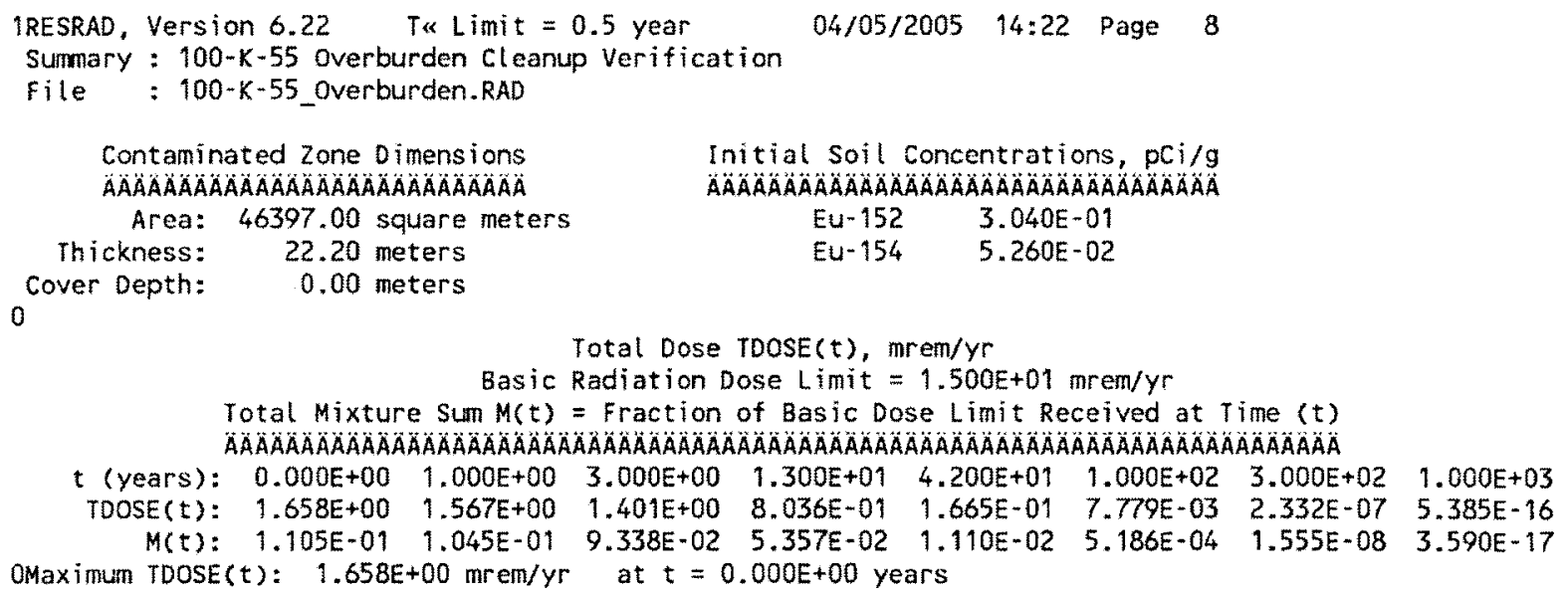


CVP-2005-00006

Rev. 0

\section{RESRAD INPUT PARAMETERS FOR THE} 100-K-56:1 SHALLOW ZONE 
CVP-2005-00006

Rev. 0

C-32 
CVP-2005-00006

Rev. 0

IRESRAD, Version 6.22 T\& Limit $=0.5$ year $07 / 19 / 2005 \quad 07: 23$ Page 1

Sumary : 100-K-56 Pipeline cleanup verification RESRAD Calculation

File: :100-K-56_Shallow_Zone.RAD

Table of Contents

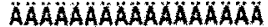

Part I: Mixture Sums and Single Radionuclide Guidel ines

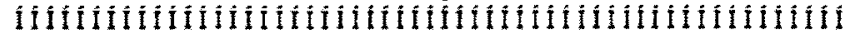

Dose Conversion Factor (and Related) Parameter Sumary ... 2

Site-specific Parameter sumary ..................... 3

Sumnary of Pathway selections .................... 7

Contaminated Zone and Totat Dose Surmary ............. 8

Total Dose Components

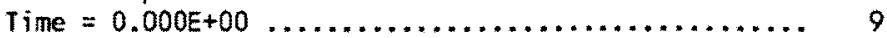

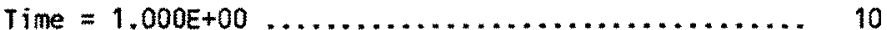

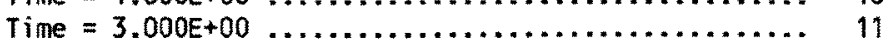

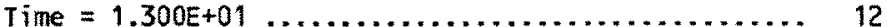

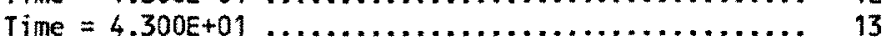

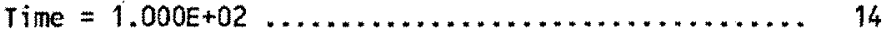

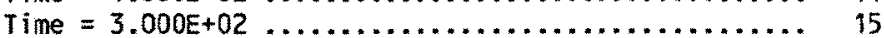

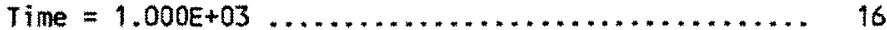

Dose/Source Ratios Sumed over All Pathways ............ 17

Single Radionucl ide Soil Guidel ines .................. 17

Dose Per Nuclide Sumed Over All Pathways ............... 18

Soil Concentration Per Nuclide $\ldots \ldots \ldots \ldots \ldots \ldots \ldots \ldots . \ldots \ldots$ 
CVP-2005-00006

Rev. 0

1RESRAD, Version 6.22 T* Limit $=0.5$ year $07 / 19 / 2005 \quad 07: 23$ Page 2

Summary : 100-K-56 Pipel ine Cleanup Verification RESRAD Calculation

File: $100-k-56$ shall low_Zone.RAD

Dose Conversion Factor (and Related) Parameter Sumary File: HEAST 2001 Morbidity

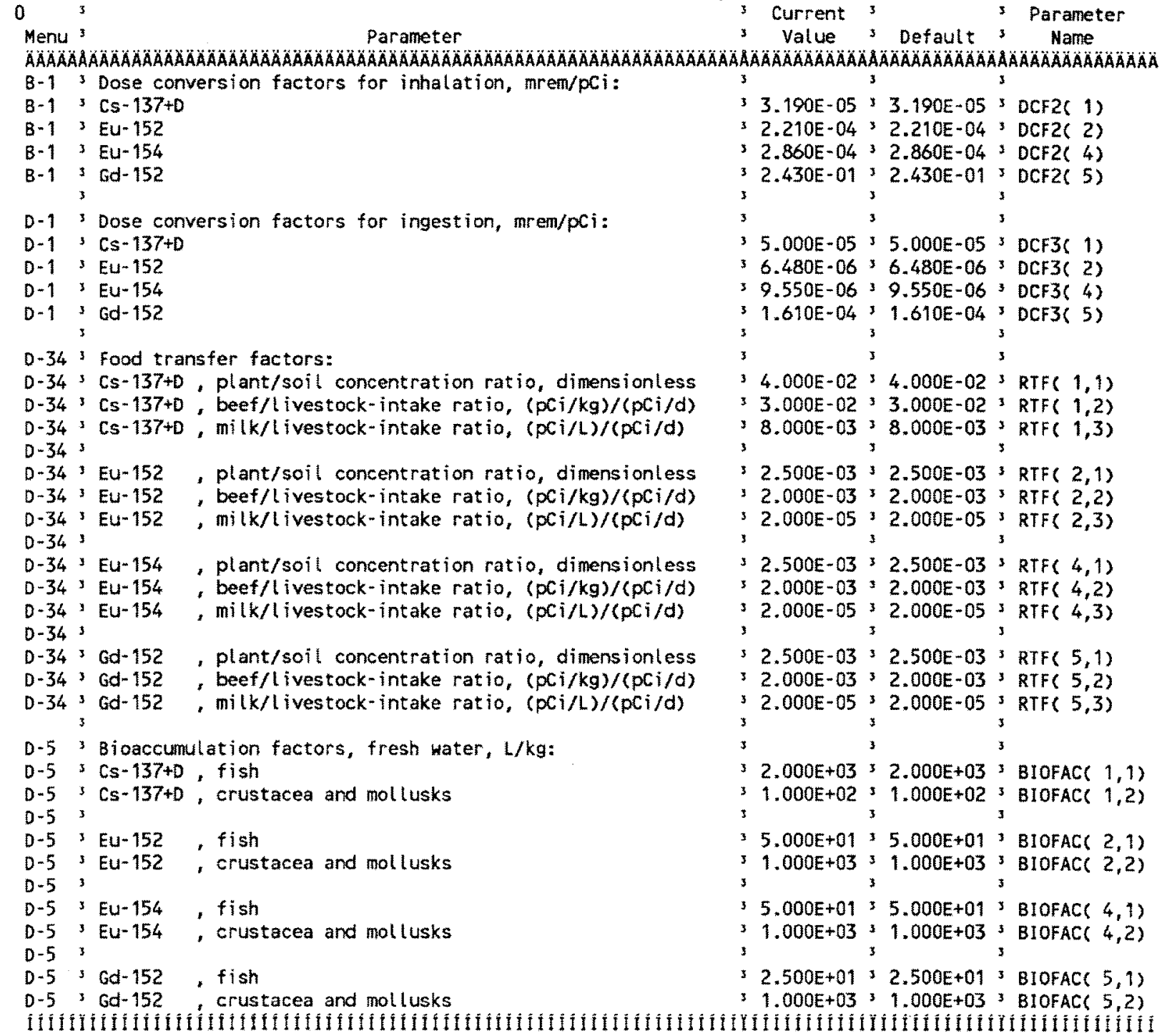


CVP-2005-00006

Rev. 0

1RESRAD, version 6.22 T\& Limit $=0.5$ year $07 / 19 / 2005 \quad 07: 23$ page 3 Sumary : 100-K-56 Pipeline Cleanup Verification RESRAD Calculation

File : 100-K-56_Shallow_zone.RAD

0

Mens " Parameter

Site-Specific Paraneter Sumary

I User 3 , Input

User " " Default "

Used by RESRAD $\quad$ Parameter

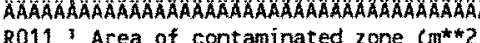

R011 Thickness of contaminated zone (m)

R011' Length parallel to aquifer flow (m)

R011'Basic radiation dose limit (mrem/yr)

R011 Time since placement of material (yr)

Ro11 Times for calculations (yr)

R011' Times for calculations (yr)

R011' Times for calculations $(y r)$

R011' Times for calculations $(y r)$

R011' Times for calculations (yr)

R011' Times for calculations ( $y r)$

R011' Times for calculations $(y r)$

R011'Times for calculations (yr)

R011 Times for calculations $\left(y^{m}\right)$

3

R012 Initial principal radionuclide $(p C i / g)=\operatorname{cs-137}, 6.100 E-01,0.000 E+00$

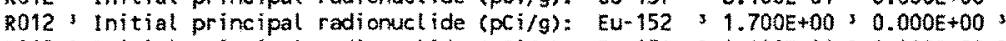

R012 Initial principal radionuclide $(\alpha \mathrm{Ci} / \mathrm{g})$ : $\mathrm{EU}-154,1.400 \mathrm{E}-01 ; 0.000 E+00$

R012 Concentration in groundwater (PCi/L): $C s-137$ mot used; $0.000 E+00$

R012 Concentration in groundwater (OCi/L): Eu-152, not used $30.000 E+00$

R012. Concentration in groundwater (PCi/L): Eu-154, not used $0.000 E+00$,

,

R013 Cover depth (m)

R013. Density of cover material $\left(\mathrm{g} / \mathrm{cm}^{* * 3}\right)$

R013' Cover depth erosion rate $(\mathrm{m} / \mathrm{yr})$

R013 Density of contaminated zone $\left(\mathrm{g} / \mathrm{cm}^{* * 3}\right)$

AAAAAAAAAAAAAAANAAAAÄAAAAA
$31.984 \mathrm{E}+04,1.000 \mathrm{E}+04$,

$34.600 \mathrm{E}+00 \div 2.000 \mathrm{E}+00$

3 $2.160 \mathrm{E}+02+1.000 \mathrm{E}+02$

$1.500 \mathrm{E}+0132.500 \mathrm{E}+01$

$31.000 E+0031.000 E+00$

$3.000 E+00 ; 3.000 E+00$

- $1.300 \mathrm{E}+01 \times 1.000 \mathrm{E}+013$

$4.300 \mathrm{E}+0133.000 \mathrm{E}+01$

$1.000 E+02 \div 1.000 E+02$

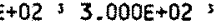

$1.000 E+03,1.000 E+03$

3 not used $30.000 E+00$ ?

not used $30.000 E+00$ ?

(If different from user input)

Name

R013 Contaminated zone total porosity $\quad 34,000 E-0134.000 E-01$

R013 Contaminated zone field capacity $32.000 E-0132.000 E-013$

R013 Contaminated zone hydraulic conductivity (m/Yr) , 2.500E $+02,1.000 \mathrm{E}+013$

$34.050 E+00 \div 5.300 E+00$

R013 Average armual wind speed $(\mathrm{m} / \mathrm{sec})$

$33.400 E+0032.000 E+00$

3 not used $38.000 E+00$

3. $9.100 \mathrm{E}-01^{3}$ 5.000E-01:

$1.600 \mathrm{E}-01 \geqslant 1.000 \mathrm{E}+00$

$7.600 \mathrm{E}-0132.000 \mathrm{E}-01$

3 overhead 3 overhead 3

2.000E-01;2.000E-01 s

R013 3 Irrigation $(\mathrm{m} / \mathrm{yr}$

R013: Runoff coefficient

R013 Watershed area for nearby stream or pond $\left(m^{* * 2}\right)$

R013: Accuracy for water/soil computations

5

R014 Density of saturated zone ( $\left.\mathrm{g} / \mathrm{cm}^{* * 3}\right)$

R014 : Saturated zone total porosity

R014: Saturated zone effective porosity

R014 Saturated zone field capacity

R014 Saturated zone hydraulic conductivity (m/yr)

R014 Saturated zone hydraulic gradient

R014 * Saturated zone b paraneter

R014 Water table drop rate (m/yr)

R014 Well pump intake depth (m below water table)

$1.000 E+06 ; 1.000 E+06$

$1.000 E-0331.000 E-03$

$1.600 E+00 ; 1.500 E+00$

4.000E-01'4.000E-01

$2.500 E-01: 2.000 E-01$ s

$1.500 E-01=2.000 E-01$

$35.530 E+03 \div 1.000 E+02$

$31.250 E-0332.000 E-02$,

$3.050 E+00 \div 5.300 E+00$

$1.000 \mathrm{E}-03+1.000 \mathrm{E}-03$

$3.600 E+0031.000 E+01$

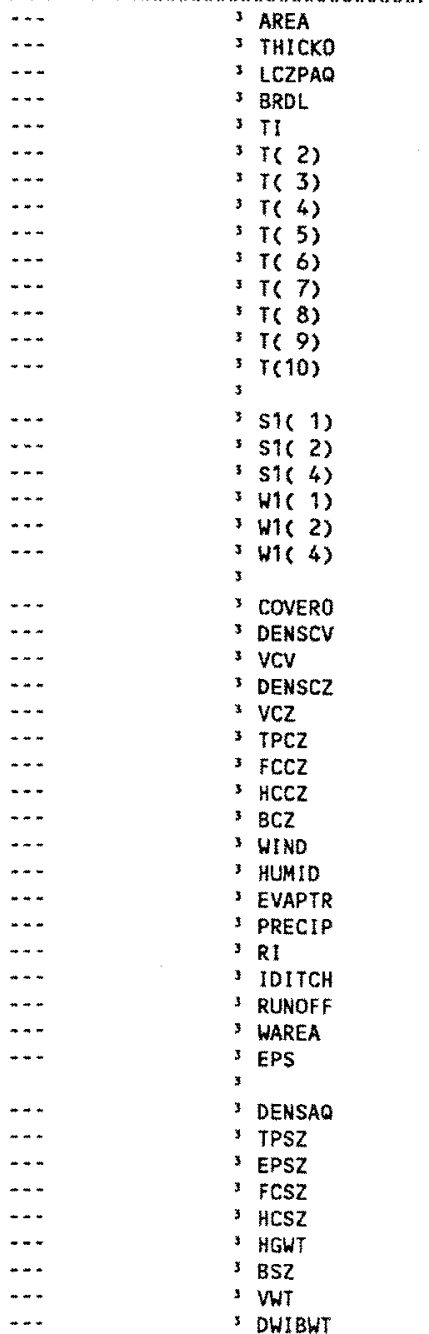


CVP-2005-00006

Rev. 0

1RESRAD, Version 6.22 T« Limit $=0.5$ year $07 / 19 / 2005$ 07:23 Page 4
Sumary $=100-\mathrm{K}-56$ Pipeline Cleanup Verification RESRAD Calculation

file: 100-K-56_Shallowzone.RAD

\begin{tabular}{|c|c|c|c|c|c|c|c|}
\hline \multicolumn{8}{|c|}{ Site-Specific Parameter Sumnary (continued) } \\
\hline 0 & 3 & User & 3 & 3 & Used by RESRAD & s & Parameter \\
\hline Menu & Parameter & $3 \quad$ Input & 3 Default & $3(1+0$ & different from user input) & 3 & Name \\
\hline AAAAAX & AAAAAAAAAAAAAAAAAAAAAAAAAAAAAAAAAAAAAAAAAAAAAAAAAA & & & & 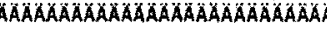 & & AAAAAAAAAA \\
\hline R014 & Model: Nondispersion (ND) or Mass-Balance (MB) & No & 3 ND & 3 & $\cdots$ & 3 & MODEL \\
\hline R014 & Well pumping rate $\left(m^{* * 3 / y r)}\right.$ & $\therefore 2.500 \mathrm{E}+02$ & $=2.500 \mathrm{E}+02$ & 3 & +- & 3 & UH \\
\hline & & 3 & 3 & 3 & & 5 & \\
\hline R015 & - Number of unsaturated zone strata & 11 & s 1 & 3 & $\cdots$ & $s$ & NS \\
\hline R015 & Unsat. zone 1, thickness (m) & $1.110 \mathrm{E}+01$ & $34.000 \mathrm{E}+00$ & 3 & $\cdots$ & 3 & $H(1)$ \\
\hline R015 & "Unsat. zone 1, soil density $\left(9 / \mathrm{cm}^{* *} 3\right)$ & $1.600 \mathrm{E}+00$ & $31.500 E+00$ & 3 & $\cdots$ & 3 & DENSUZ(1) \\
\hline R015 & 'Unsat. zone 1 , total porosity & $4.000 \mathrm{E}-01$ & $=4.000 E-01$ & $\Sigma$ &.- & 3 & TPUZ(1) \\
\hline R015 & "Unsat. zone 1, effective porosity & $2.500 \mathrm{E}-01$ & $32.000 \mathrm{E}-01$ & 3 & $m$ & 3 & EPUZ(1) \\
\hline R015 & ' Unsat. zone 1, field capacity & $31.500 \mathrm{E}-01$ & $32.000 E-01$ & 3 & $\cdots$ & 3 & FCUZ(1) \\
\hline R015 & ' Unsat. zone 1, soil-specific b parameter & $=4.050 E+00$ & $=5.300 E+00$ & 2 & $\cdots$ & 3 & BUz(1) \\
\hline R015 & Unsat. zone 1, hydraulic conductivity $(\mathrm{m} / \mathrm{yr})$ & $2.500 \mathrm{E}+02$ & $31.000 \mathrm{E}+01$ & 3 & $\cdots$ & 3 & HCUZ(1) \\
\hline & 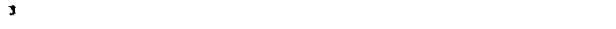 & 3 & 3 & 3 & & 3 & \\
\hline R016 & Distribution coefficients for Cs-137 & 3 & 3 & 3 & & 3 & \\
\hline R016 & 3 Contaminated zone $\left(\mathrm{cm}^{* * 3 / 9)}\right.$ & $5.000 E+01$ & $31.000 E+03$ & 3 & $\cdots$ & 3 & DCNUCC $(1)$ \\
\hline$R 016$ & Unsaturated zone $1\left(\mathrm{~cm}^{* * 3 / 9}\right)$ & $5.000 E+01$ & $31.000 E+03$ & 3 & $\cdots$ & 3 & $\operatorname{DCNUCU}(1,1)$ \\
\hline R016 & Saturated zone $\left(\mathrm{cm}^{* * 3 / 9}\right)$ & $5.000 E+01$ & $=1.000 E+03$ & 3 & $\cdots$ & 3 & DCNUCS( 1) \\
\hline R016 & Leach rate $\left(/ y^{r}\right)$ & $30.000 E+00$ & $=0.000 E+00$ & 3 & $2.166 \mathrm{E}-04$ & 3 & ALEACH ( 1$)$ \\
\hline R016 & Solubility constant & $=0.000 E+00$ & $30.000 E+00$ & 3 & not used & 3 & SOLUBK $(1)$ \\
\hline & 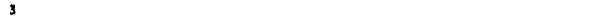 & 3 & 3 & 3 & & 3 & \\
\hline R016 & Distribution coefficients for Eu- 152 & $x$ & 3 & 3 & & 3 & \\
\hline R016 & Contaminated zone $\left(\mathrm{cm}^{* k 3 / \mathrm{g})}\right.$ & $2.000 \mathrm{E}+02$ & $3-1.000 \mathrm{E}+00$ & 3 & $\cdots$ & 3 & DCNUCC ( 2) \\
\hline R016 & Unsaturated zone $1\left(\mathrm{~cm}^{* * 3 / 9}\right)$ & $2.000 E+02$ & $3-1.000 E+00$ & 3 & $\cdots$ & 3 & DCNUCU $(2,1)$ \\
\hline R016 & Saturated zone $\left(\mathrm{cm}^{* * 3 / \mathrm{g})}\right.$ & $32.000 E+02$ & $3-1.000 E+00$ & 3 & $\cdots$ & 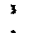 & DCNUCS $(2)$ \\
\hline R016 & Leach rate $\left(/ y^{\prime}\right)$ & $=0.000 \mathrm{E}+00$ & $0.000 E+00$ & 3 & $5.426 E-05$ & 3 & ALEACH ( 2) \\
\hline R016 & Solubility constant & $0.000 E+00$ & $=0.000 E+00$ & 3 & not used & 3 & SOLUBK( 2$)$ \\
\hline & 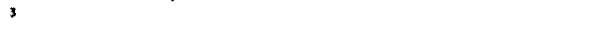 & $*$ & 3 & 1 & & 3 & \\
\hline R016 & Distribution coefficients for Eu- 154 & 3 & 3 & 3 & & 3 & \\
\hline R016 & $3 \quad$ Contaminated zone $\left(\mathrm{cm}^{* \star 3} / \mathrm{g}\right)$ & $2.000 E+02$ & $3-1.000 \mathrm{E} \div 00$ & 3 & $\cdots$ & + & Denuec $(4)$ \\
\hline R016 & Unsaturated zone $1\left(\mathrm{~cm}^{* * 3 / g}\right)$ & $2.000 E+02$ & $3-1.000 E+00$ & 3 & $\cdots$ & 3 & DCNUCU 4,1$)$ \\
\hline R016 & Saturated zone $\left(\mathrm{cm}^{\star *} 3 / \mathrm{g}\right)$ & $2.000 E+02$ & $1-1.000 E+00$ & 3 & $-\cdots$ & 1 & DCNUCS( 4$)$ \\
\hline R016 & Leach rate $\left(/ y^{r}\right)$ & $0.000 E+00$ & $=0.000 E+00$ & 3 & $5.426 E-05$ & * & ALEACH ( 4) \\
\hline R016 & Solubil ity constant & $0.000 E+00$ & $0.000 E+00$ & 3 & not used & 3 & SOLUBK $(4)$ \\
\hline & 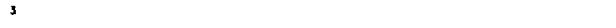 & 5 & 3 & 3 & & 1 & \\
\hline R016 & Distribution coefficients for daughter $6 d-152$ & 3 & 3 & 3 & & $*$ & \\
\hline R016 & Contaminated zone $\left(\mathrm{cm}^{* * 3 / \mathrm{g}}\right)$ & $3-1.000 E+00$ & $3-1.000 E+00$ & 3 & $8.249 E+02$ & 3 & DCNUCC 5 ) \\
\hline R016 & Unsaturated zone $1\left(\mathrm{~cm}^{* * 3 / 9}\right)$ & $3-1.000 E+00$ & $-1.000 E+00$ & 3 & $8.249 E+02$ & 3 & DCNuCu 5,1$)$ \\
\hline R016 & Saturated zone $(\mathrm{cm} * * 3 / 9)$ & $3-1.000 E+00$ & $s-1.000 E+00$ & 3 & $8.249 \mathrm{E}+02$ & $y$ & DCNUCS $(5)$ \\
\hline R016 & Leach rate $(/ y r)$ & $0.000 E+00$ & $0.000 E+00$ & 3 & $1.316 E-05$ & 3 & ALEACH ( 5) \\
\hline R016 & Solubility constant & $0.000 \mathrm{E}+00$ & $0.000 E+00$ & 3 & not used & 3 & SOLUBK( 5$)$ \\
\hline & 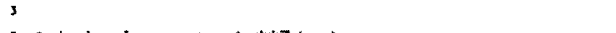 & 370000 & $\therefore$ & 3 & & 3 & \\
\hline R017 & Inhalation rate $\left(m^{* * 3 / y r}\right)$ & $7,300 \mathrm{E}+03$ & $s 8.400 E+03$ & 3 & $\cdots$ & 3 & INHALR \\
\hline R017 & - Mass loading for inhalation $\left(9 / \mathrm{m}^{\star * 3}\right)$ & $31.000 \mathrm{E}-04$ & $31.000 E-04$ & 3 & $-\infty$ & 3 & MLINH \\
\hline R017 & Exposure curation & $3.000 E+01$ & $3.000 E+01$ & 3 & $\cdots$ & 3 & EO \\
\hline 8017 & Shielding factor, inhalation & $34.000 E-01$ & $4.000 \mathrm{E}-01$ & 3 & $\cdots$ & 3 & 5HF3 \\
\hline $\mathrm{R} 017$ & Shielding factor, external gama & $8.000 \mathrm{E}-01$ & $7.000 \mathrm{E}-01$ & 3 & $+\cdots$ & 7 & SHF 1 \\
\hline R017 & Fraction of time spent indoors & $6.000 E-01$ & s 5.000E-01 & s & $\cdots$ & 3 & FIND \\
\hline 2017 & 3 Fraction of time spent outdoors (on site) & $2.000 E-01$ & $2.500 E-01$ & 3 & $m$ & 3 & FOTD \\
\hline B017 & Shape factor flag, external gamma & $1.000 E+00$ & $=1.000 E+00$ & 3 & 0 shows circular AREA. & 3 & FS \\
\hline
\end{tabular}


CVP-2005-00006

Rev. 0

IRESRAD, Version 6.22 T\& Limit $=0.5$ year $07 / 19 / 2005 \quad 07: 23$ page 5 Sumary : 100-K-56 Pipeline cleanup Verification RESRAD Calculation

File: 100-K-56 shall ow Zone. RAD Site-Specific Parameter Sumary (continued)

Menv Parameter User 3 Used by RESRAD Parameter MenU R017; Outer annular radius (m), ring : R017' Outer annular radius (m), ring 2 R017 Outer annular radius (m), ring 3 : $\mathrm{RO17}^{3}$ Outer annular radius (m), ring 4: R017' Outer annular radius (m), ring 5: R017 ${ }^{3}$ Outer annular radius (m), ring 6 : R017 7 Outer annular radius (m), ring 7: R017; Outer annular radius (m), ring 8: R017 Outer arnular radius (m), ring 9: R017: Outer arnular radius (m), ring 10: R017, Outer annular radius (m), ring 11: R017, Outer annular radius $(\mathrm{m})$, ring 12 :

RO17' Fractions of annular areas within AREA: R017' Ring

ROIT? Ring

Ro17: Ring 3

Ro17: Ring 4

Ro17: Ring 5

R017' Ring 6

R017? Ring 7

R017* Ring 8

Ro17' Ring 9

R017 : Ring 10

Ro17: Ring 11

R017: Ring 12

R018 Fruits, vegetables and grain consumption (kg/yr)

' not used : $5.000 E+01$

R018 Leafy vegetable consumption ( $\mathrm{kg} / \mathrm{yr}$ )

R018 Milk consumption ( $L / y r)$

R018 Meat and poultry consurption ( $\mathrm{kg} / \mathrm{yr})$

R018 Fish consumption ( $\mathrm{kg} / \mathrm{yr}$ )

R018 other seafood consumotion ( $\mathrm{kg} / \mathrm{yr}$ )

R018 Soil ingestion rate $(g / y r)$

RO18: Drinking water intake (L/yr)

R018 ' Contamination fraction of drinking water

R018 Contamination fraction of household water

R018 Contamination fraction of livestock water

R018 3 Contamination fraction of irrigation water

R018 Contamination fraction of aquatic food

R018 Contanination fraction of plant food

R018 Contamination fraction of meat

R018 Contamination fraction of milk

3

R019 3 Livestock fodder intake for meat ( $\mathrm{kg} / \mathrm{day}$ ) R019: Livestock fodder intake for milk (kg/day)

R019 Livestock water intake for meat (L/day)

R019 3 Livestock water intake for milk (L/day)

$\mathrm{R019}$ " Livestock soil intake $(\mathrm{kg} /$ day) not used $37.071 \mathrm{E}+01$

3 not used $30.000 E+00$

not used $=0.000 E+00^{3}$

not used $0.000 \mathrm{E}+00$,

not used $0.000 E+00$ :

3 not used $>0.000 E+00$

3 not used $30.000 E^{3}+00^{3}$

not used $10.000 E+00^{3}$

not used $0.000 \mathrm{E}+00$

not used $30.000 E+00$

not used $0.000 E+00$

not used $31.000 E+00$

not used $32.732 \mathrm{E}-01$

not used $30.000 E+00$ ?

not used, $0.000 E+00$

not used $>0.000 E+00$

3 not used $30.000 E+00$

3 not used $>0.000 E+00$ s

not used $30.000 E+00$ '

not used $0.000 E+00$

' not used $30.000 E+00$

not used $\$ 0.000 E+00$

3 not used $30.000 E+00$;

$2.700 E+00 ; 1.400 E+01$

$3.000 E+02: 9.200 E+01$

$3.600 \mathrm{E}+01 \times 6.300 \mathrm{E}+01$

$1.970 E+0135.400 E+00$

$9.000 \mathrm{E}-0139.000 \mathrm{E}-01$

, 7.300E+01 $3.650 E+01$ '

$7.300 \mathrm{E}+02 \times 5.100 \mathrm{E}+02$

$=1.000 E+0031.000 E+00$

' not used $11.000 E+00$

$1.000 \mathrm{E}+0031.000 \mathrm{E}+00$

$31.000 E+00 ; 1.000 E+00$

, 5.000E-01 $5.000 \mathrm{E}-01$

3-1

3. -1

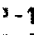

$s-1$

-

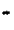

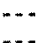

$\cdots$

-.-

$\cdots$

$\cdots$

$\cdots$

$\cdots$

$\cdots$

$\cdots$

$6.800 E+01 ; 6.800 E+01$

$3.500 E+01 ; 5.500 E+01$

3 5.000E+01'5.000E+01

$11.600 E+02: 1.600 E+02$

, 5.000E-01'5.000E-01'
- RAD_SHAPE( 1)

- RAO SHAPE( 2 )

3 RAD SHAPE( 3 )

- RAD SHAPE ( 4)

RAD SHAPE ( 5)

RAD_SHAPE ( 6)

RAD SHAPE ( 7)

RAD SHAPE ( 8)

RAD_SHAPE ( 9)

RAD SHAPE (10)

RAD_SHAPE (11)

RAD_SHAPE(12)

FRACAC 1)

FRACA( 2)

3 FRACAC 3)

Fraca( 4)

3 FRACA ( 5)

FRACA ( 6)

3 FRACA (7)

- FRACa( 8)

FRACAC 9)

FRACA(10)

- FRACA(11)

FRACA(12)

DIET(1)

DIET(2)

DIET (3)

3 DIET(4)

DIET (5)

DIET(6)

SOIL

DWI

FDW

FHHW

3 FLW

FIRH

FR9

FPLANT

FMEAT

FMILK

LFI5

'LF16

LWI5

LU16 
IRESRAD, version 6.22 T\& Limit $=0.5$ year $07 / 19 / 2005 \quad 07: 23$ Page 6 Sumary : 100-K-56 Pipeline Cleanup Verification RESRAD Calculation

file: $100-K-56$ shallow_Zone.RAD

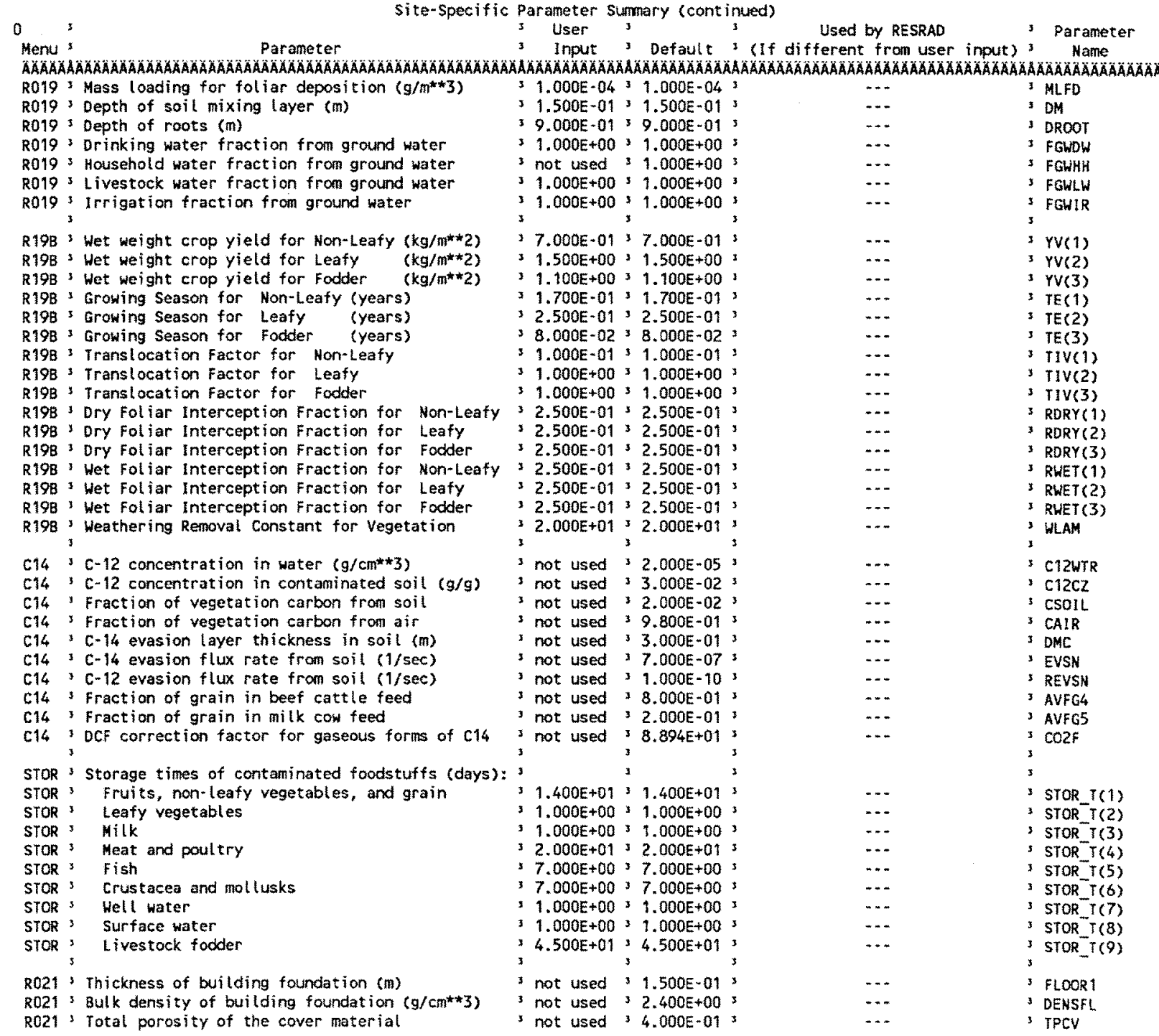


CVP-2005-00006

IRESRAD, Version 6.22 T\& Limit $=0.5$ year $07 / 19 / 2005 \quad 07: 23$ Page 7

Sumary : 100-K-56 Pipetine Cleanup Verification RESRAD Calculation

File: 100-K-56_Shallow_Zone.RAO

0 s

Site-Specific Parameter Sumary (continued)

Menu'

Parameter

Parameter Sumbry (continued)

Used by RESRAD

- Paraneter

AAAAAAAA

R021 Total porosity of the building foundation 3 not used $31,000 E-01$

R021 Volumetric water content of the cover material s not used $35.000 E-02$

R021 Volumetric water content of the foundation, not used $33.000 E-02{ }^{3}$

R021 Diffusion coefficient for radon gas (m/sec):

R021 in cover material

RO21 in foundation material

RO21 in contaminated zone soil

R021 Radon vertical dimension of mixing $(\mathrm{m})$

R021 Average building air exchange rate (1/hr)

R021 3 Height of the butlding (room) $(m)$

R021 Building interior area factor

R021 Building depth below ground surface (n)

not used 3 , 3.000E-02,

not used 32.000 E-06?

'not used $3.000 E-07$ '

3 not used 32.000 E-06?

not used $2.000 E+00^{3}$

not used 3 5.000E-013

3 not used $32.500 E+00^{3}$

3 not used $0.000 \mathrm{E}+00$ :

not used $3-1.000 \mathrm{E}+00^{3}$

not used $32.500 E-013$

not used; $1.500 \mathrm{E}-01$;

20213

TIIL N Number of graphical time points

TITL 3 Maximum number of integration points for dose

$\begin{array}{lrlll}3 & 32 & 3 & \ldots & 3 \\ 3 & 1 & 3 & \ldots & 3\end{array}$

\begin{tabular}{|c|c|c|}
\hline$\cdots$ & 3 & TPFL \\
\hline$m$ & 3 & PH2OCV \\
\hline$\cdots$ & 3 & PHZOFL \\
\hline- & J & DIFCV \\
\hline & 3 & DIFFL \\
\hline & 3 & DIFCZ \\
\hline & 3 & HAIX \\
\hline . & 3 & REXG \\
\hline 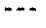 & 3 & HRM \\
\hline$\cdots$ & 3 & FAI \\
\hline 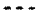 & 3 & DMFL \\
\hline$\cdots$ & 3 & EMANA(1) \\
\hline - & $y$ & EMANA (2) \\
\hline . & 3 & NPTS \\
\hline & 3 & LYMAX \\
\hline
\end{tabular}

3 KYMAX

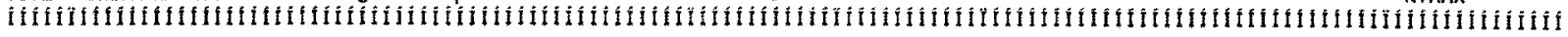

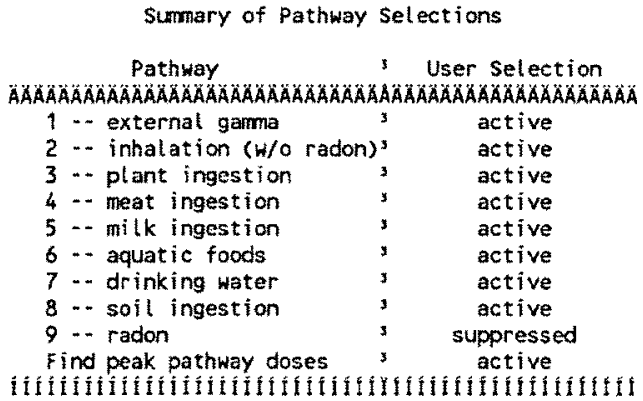


CVP-2005-00006

Rev. 0

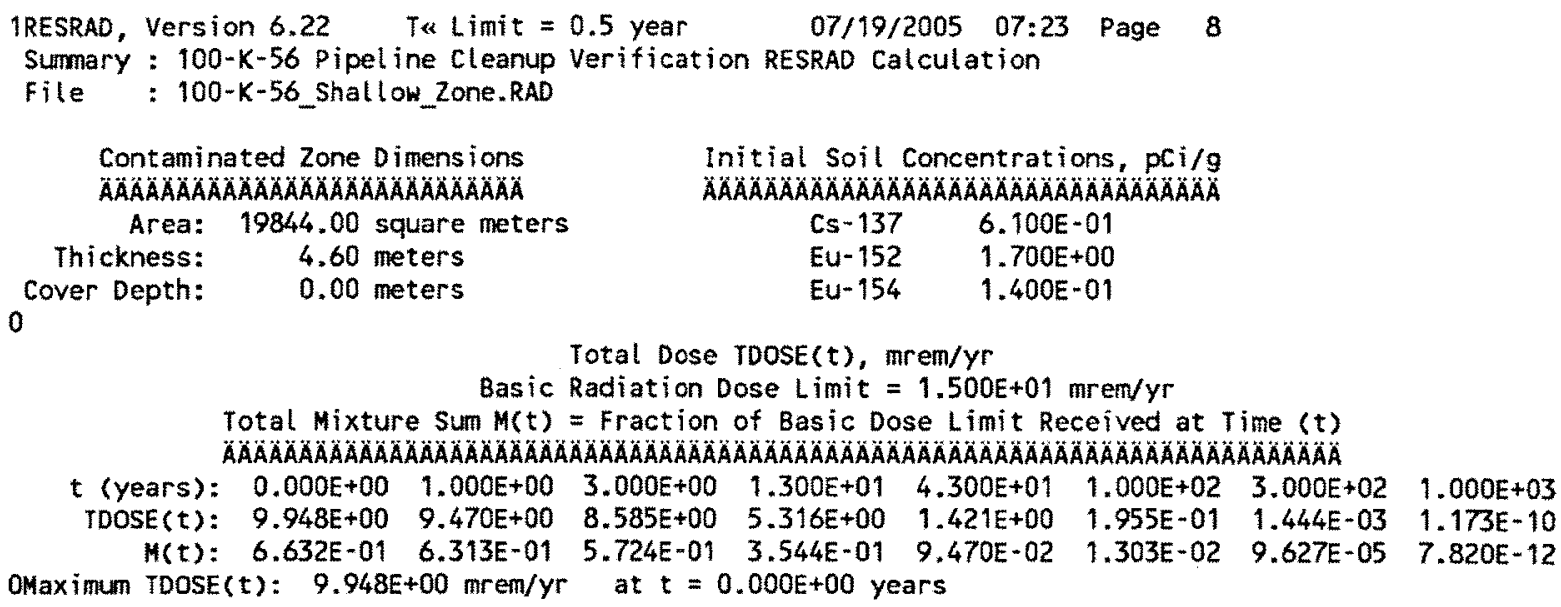


CVP-2005-00006

Rev. 0

RESRAD INPUT PARAMETERS FOR THE 100-K-56:1 DEEP ZONE 
CVP-2005-00006

Rev. 0

C-42 
CVP-2005-00006

Rev. 0

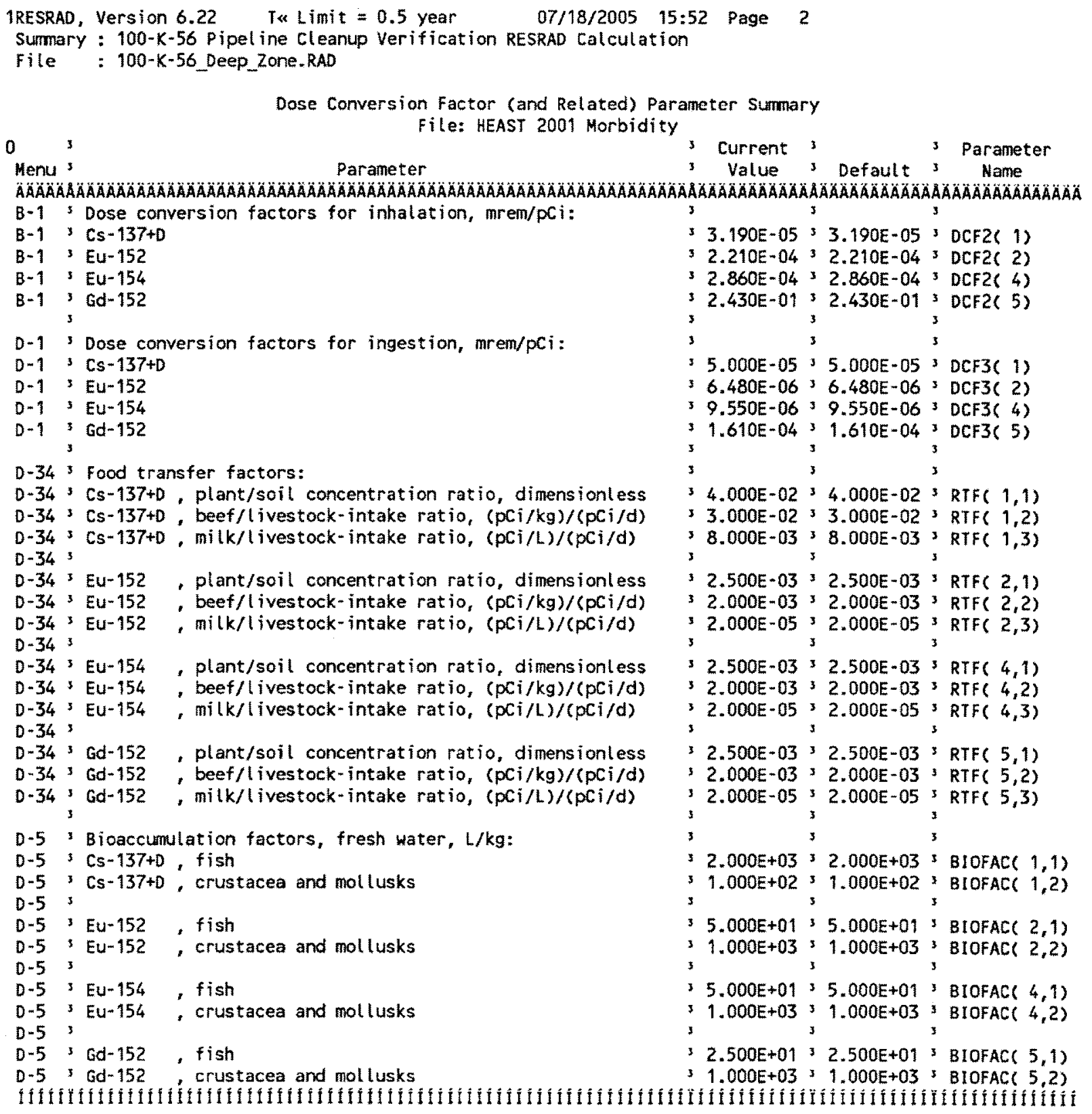


CVP-2005-00006

Rev. 0

IRESRAD, Version $6.22 \quad$ T* Limit $=0.5$ year $\quad 07 / 18 / 2005 \quad 15: 52$ Page 3
Sumary: $100-K-56$ Pipeline Cleanup Verjfication RESRAD Calculation

file : 100-K-56_Deep_zone.RAD

0

Site-Specific Parameter sumary

Menu

Parameter

3 User

3

Used by RESRAD

Parameter

AAAAAAAA

R011' Area of contaminated zone $\left(m^{* *} 2\right)$

R011 Thickness of contaminated zone (m)

R011 Length parallel to aquifer flow (m)

R011 Basic radiation dose limit (mrem/yr)

R011 Time since placement of material $(y r)$

R011 Times for calculations ( $y r)$

R011'Times for calculations (yr)

R011 Times for calculations (yr)

R011 Times for calculations $(y r)$

R011 Times for calculations (yr)

R011 Times for calculations (yr)

R011 Tines for calculations (yr)

R011' Times for calculations ( $y r)$

R011 Times for calculations $(y r)$

*

$31.13 E+04: 1.000 E+04$

$31.110 E+01,2.000 E+00$

$2.160 E+02,1.000 E+02$

$1.500 E+01,2.500 E+01+$

$30.000 E+00>0.000 E+00$

$1.000 E+00: 1.000 E+00$

$3.000 E+00^{3} 3.000 E+00^{3}$

$1.300 E+01=1.000 E+01$

$34.300 E+01>3.000 E+01$

$31,000 \mathrm{E}+02,1,000 \mathrm{E}+02$,

$3.000 E+02 \times 3.000 E+02$

$1.000 E+03 ; 1.000 E+03$

3 not used $30,000 E+00$

3 not used, $0.000 E+00$

R012 Initial principal radionuclide $(p c i / g): \operatorname{cs}-137,1.320 E+01,0.000 E+00$

R012 Initial principal radionuclide $(\mathrm{DC} / \mathrm{g}):$ Eu-152 $31.370 \mathrm{E}+0130.000 \mathrm{E}+00$

R012, Initial principal radionuclide (PCi/g): Eu-154;1.870E+00 $=0.000 E+00 *$

$\mathrm{R012}$, Cancentration in groundwater (pCi/L): $\mathrm{CS}_{5}-137$ ' not used, $0.000 \mathrm{E}+00$

R012 : Concentration in groundwater ( $\mathrm{PC} j / L$ ): Eu-152 s not used $10.000 \mathrm{E}+00$,

R012" Concentration in groundwater (PC/L): Eu-154' not used $0.000 \mathrm{E}+00$,

R013 3 Cover depth (m)

R013 Density of cover material $\left(\mathrm{g} / \mathrm{cm}^{* * 3}\right)$

R013 3 Cover depth erosion rate (m/yr)

R013 Density of contaminated zone $(\mathrm{g} / \mathrm{cm} * * 3)$

R013, Contaminated zone erosion rate $(\mathrm{m} / \mathrm{yr})$

Ro13 Contaminated zone total porosity

R013 : Contaninated zone field capacity

R013' Contaminated zone hydraulic conductivity ( $m / y r)$

$R 013^{3}$ Contaminated zone b parameter

R013 Average annual uind speed (m/sec)

Ro13, Humidity in air $\left(g / m^{* * 3}\right)$

R013 Evapotranspiration coefficient

R013 Precipitation (m/yr)

R013? Irrigation ( $\mathrm{m} / \mathrm{yr}$ )

R013, Irrigation mode

Ro13 Runoff coefficient

$4.600 E+00 ; 0.000 E+00$

$1.600 \mathrm{E}+00,1.500 \mathrm{E}+00$

$1.000 \mathrm{E}-03 \times 1.000 \mathrm{E}-03$,

$1.600 \mathrm{E}+00 \times 1.500 \mathrm{E}+00$

$31.000 \mathrm{E}-03,1.000 \mathrm{E}-03$

, 4.000E-01 $3,000 E-01$

$2.000 \mathrm{E}-01,2.000 \mathrm{E}-013$

$2.500 E+02 \times 1.000 E+01$

$4.050 \mathrm{E}+00 \div 5.300 \mathrm{E}+00$

$3.400 \mathrm{E}+00 \div 2,000 \mathrm{E}+00$

not used $>8.000 \mathrm{E}+00$

$9.100 E-01$ s $5.000 E-01$

$1.600 E-01 ; 1.000 E+00$

$7.600 E-0132.000 E-01$

3 overhead s overhead

$2.000 E-0132.000 E-01$

Watershed area for nearby stream or pond $\left(m^{* *} 2\right), 1.000 \mathrm{E}+06,1.000 \mathrm{E}+06$,

R013 Accuracy for water/soil computations

R014 3 Density of saturated zone $\left(9 / \mathrm{cm}^{* * 3}\right)$

R014 Saturated zone total porosity

R014 3 Saturated zone effective porosity

R014 3 Saturated zone field capacity

R014 S Saturated zone hydraul ic conductivity $(\mathrm{m} / \mathrm{yr})$

R014' Saturated zone hyoraulic gradient

R014 S Saturated zone b parameter

R014 Water table drop rate ( $\mathrm{m} / \mathrm{yr}$ )

R014 Well pump intake depth (n below water table) $\rightarrow 1.000 E-03=1.000 E-03$

$31.600 \mathrm{E}+00: 1.500 \mathrm{E}+00$;

4.000E-01 4.000E-01

$32.500 E-0132.000 E-01$

$1.500 \mathrm{E}-0132.000 \mathrm{E}-013$

$35.530 E+03,1.000 E+02$

$3.250 E-03 ; 2.000 E-02$

$34.050 E+00>5.300 E+00:$

$3,000 E-03 ; 1.000 E-03$;

$34.600 E+00 ; 1.000 E+01 ;$

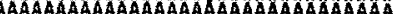

*-* 3 AREA

". THICKO

ICZPAO

...

- 3 T $(2)$

$T(3)$

, $T(4)$

$\cdots+3(5)$

$\cdots 3 T(6)$

$3 T(8)$

T(

$T(10)$

s1( 1$)$

S1( 2)

si( 4$)$

Hi( 1$)$

- Wi( 2)

W1( 4$)$

COVERO

OENSCV

$3 \mathrm{VCV}$

DENSCZ

$3 \mathrm{VCZ}$

- TPCZ

FCCZ

$3 \mathrm{HCCZ}$

$3 \mathrm{SCZ}$

J

J HUNID

EVAPTR

PRECIP

* RI

IDITCH

* RUNOFF

3 WAREA

EPS

DENSAQ

I TPSZ

3 EPSZ

FCSZ

HCSZ

HGWT

3 BSZ

3 VWT

$\checkmark$ DUIBUT 
CVP-2005-00006

Rev. 0

IRESRAD, version 6.22 Tu Limit $=0.5$ year $07 / 18 / 200515: 52$ Page 4

Sumary : 100-K-56 Pipeline Cleanup Verification RESRAD Calculation

File: $100-x+56$ _oeep_zone.RAD

\begin{tabular}{|c|c|}
\hline & 3 \\
\hline $\begin{array}{l}\text { Menu } \\
A A A A A\end{array}$ & 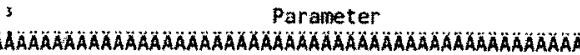 \\
\hline $\begin{array}{l}\mathrm{RO} 14 \\
\mathrm{R} 014\end{array}$ & $\begin{array}{l}\text { Model: Nondispersion (ND) or Mass-Balance (MB) } \\
\text { Well pumping rate }\left(m^{* * 3 / y r)}\right.\end{array}$ \\
\hline R015 & Number of unsaturated zone strata \\
\hline $\begin{array}{l}\text { R016 } \\
\text { R016 } \\
\text { R016 } \\
\text { R016 } \\
\text { R016 }\end{array}$ & $\begin{array}{l}\text { Distribution coefticients for } \mathrm{cs-137} \\
\text { Contaminated zone }\left(\mathrm{cm}^{* * 3 / g}\right) \\
\text { Saturated zone }\left(\mathrm{cm}^{* * 3 / g)}\right. \\
\text { Leach rate }(/ \mathrm{yr}) \\
\text {, Solubility constant }\end{array}$ \\
\hline $\begin{array}{l}R 016 \\
R 016 \\
R 016 \\
\text { R016 } \\
\text { R016 }\end{array}$ & $\begin{array}{l}\text { Distribution coefficients for Eu*152 } \\
\text { Contaminated zone }\left(\mathrm{cm}^{* * 3 / g}\right) \\
\text { Saturated zone }\left(\mathrm{cm}^{* * 3 / g)}\right. \\
\text { Leach rate }(/ y r) \\
\text { Solubility constant }\end{array}$ \\
\hline $\begin{array}{l}R 016 \\
R 016 \\
R 016 \\
R 016 \\
R 016\end{array}$ & $\begin{array}{l}\text { Oistribution coefficients for Eu-154 } \\
\text { Contaminated zone }(\mathrm{cm} * * 3 / 9) \\
\text { Saturated zone }\left(\mathrm{cm}^{* * 3 / 9)}\right. \\
\text { Leach rate (/yr) } \\
\text { Solubility constant }\end{array}$ \\
\hline $\begin{array}{l}R 016 \\
R 016 \\
R 016 \\
R 016 \\
R 016\end{array}$ & $\begin{array}{l}\text { Distribution coefficients for daughter fd-152 } \\
\text { Contaminated zone }\left(\mathrm{cm}^{* * 3 / g)}\right. \\
\text { Saturated zone }\left(\mathrm{cm}^{* * 3 / g)}\right. \\
\text { Leach rate }(/ \mathrm{yr}) \\
\text { Solubility constant } \\
\text {, }\end{array}$ \\
\hline $\begin{array}{l}R 017 \\
R 017 \\
R 017 \\
R 017 \\
R 017 \\
R 017 \\
R 017 \\
R 017\end{array}$ & 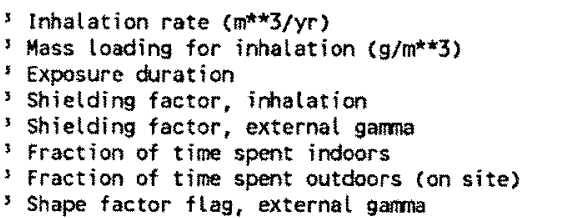 \\
\hline
\end{tabular}

Parameter Sumary (continued)

3 User 3 Used by RESRAD 3 , Parameter

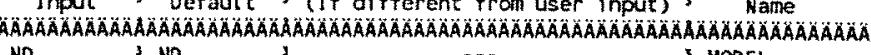

3 ND 3 NO

$2.500 E+02,2.500 E+02$

0 ,

33

$5.000 E+0131.000 E+03$

$5.000 E+0131.000 E+03$;

$0.000 E+00 ; 0.000 E+00$

$0.000 E+00,0.000 E+00$

$2.000 E+02+1.000 E+00$

$2.000 E+023-1.000 E+00$

$0.000 E+00 \div 0.000 E+00$

$0.000 E+00=0.000 E+00$

$2.000 E+02^{3}-1.000 E+00^{3}$

$2.000 E+02=1.000 E+00$

$0.000 \mathrm{E}+00=0.000 \mathrm{E}+00$

$0.000 E+00: 0.000 E+00$ ?

3

$-1.000 E+003-1.000 E+00$

$3-1.000 E+003-1.000 E+00$ s

$0.000 E+00>0.000 E+00$

$30.000 E+00 \times 0.000 E+00$

$7.300 E+03 \times 8.400 E+03$

$31.000 E-04^{3} 1.000 E-04$

$33.000 E+0133.000 E+01$,

, 4.000E-013 4.000E-01,

8.000 E-01; $7.000 E-01$,

$6.000 E-0135.000 E-01$ '

2.000E-01 $32.500 E-01$,

$31.000 \mathrm{E}+00 \times 1.000 \mathrm{E}+00$,

HODEL

$\ldots$ UW

... NS

1

…

8.978E- OS

not used

DCNucce 1$)$

DCNUCS (1)

ALEACH 1 )

SOLUBKC 1)

Denucc( 2)

2.249E-05

3 DCNUCS( 2)

ALEACH (2)

SOLUBK ( 2)

not used

DenucC (4)

DCNUCS (4)

5 ALEACH (4)

$2.249 E-05$

not used

3 SOLUBK ( 4)

$8.249 E+02$

3 DCNUCC( 5 )

$83495+02 \quad 3$ DCNUCS $(5)$

5.455E-06 3 ALEACH ( 5 )

not used 3 SOLUBK 5 )

I NHALR

-.

$\cdots$ J ED

-.. 3 SHF3

-.. $\quad$ SHE1

3 FIND

FIND

$>0$ shows circular AREA. 3 FS 
IRESRAD, Version 6.22 Tu Limit $=0.5$ year $07 / 18 / 2005 \quad 15: 52$ Page 5 Sumary : 100-K-56 Pipeline Cleanup Verification RESRAD calculation

File: $100-k-56$ Deep_zone. RAD

0

Site-specific Parameter sumary (continued)

MenU *

Parameter

Parameter Sumary (continued)

Used by RESRAD

Parameter

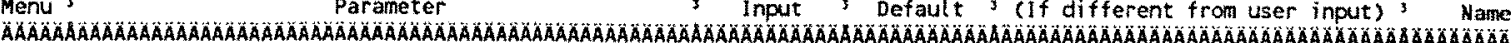

R017' Radi $i$ of shape factor array (used if FS $=-1$ ):

R017: Outer annular radius (m), ring 1:

$2017^{3}$ Outer annular radius $(m)$, ring 2:

R017' outer amular radius (m), ring 3 :

R017 5 Outer anular radius (m), ring 4:

R017' Outer annular radius (m), ring 5 :

R017 : Outer annular radius ( $m$ ), ring 6 :

R017 - Outer annular radius (m), ring 7:

R017' Outer annular radius (m), ring 8:

R017, Outer annular radius $(m)$, ring 9:

R017 S Outer annular radius (m), ring 10:

R017: Outer annular radius (m), ring 11:

R017, Duter annular radius (m), ring 12:

R017 : Fractions of annular areas within AREA:

R017' Ring 1

R017's Ring 2

R017 R Ring 3

R017 B Ring 4

R017: Ring 5

R017? Ring 6

R017' Ring 7

Ro17: Ring 8

R017 Ring 8

R017 3 ging 10

Ro17, Ring 11

R017 Ring 12

R018 Fruits, vagotobles and grain consurption (kg/yr)

R018' Leafy vegetable consumption $(\mathrm{kg} / \mathrm{yr})$

Ro18' Milk consumption (L/Yr)

R018 Heat and poultry consumption ( $\mathrm{kg} / \mathrm{yr})$

R018 3 Fish consumption $(\mathrm{kg} / \mathrm{yr}$ )

Ro18 * other seafood consumption (kg/yr)

R018' Soil ingestion rate $(g / \mathrm{y} r)$

R018' Drinking water intake (L/yr)

R018 ' Contamination fraction of drinking water

R018 Contamination fraction of household water

R018 Contamination fraction of livestock water

$R 018$ : Contamination fraction of irrigation water

$R 018$ ' Contamination fraction of aquatic food

2018 . Contamination fraction of plant food

R018' Contamination fraction of meat

2018 Contamination fraction of milk

$$
3
$$

R019' Livestock fodder intake for meat (kg/day)

R019 3 Livestock fodder intake for milk (kg/day)

R019 Livestock water intake for meat ( $L /$ day)

R019' Livestock water intake for milk (L/day)

R019' Livestock soil intake ( $\mathrm{kg} /$ day)
3 ?

not used $5.000 E+013$

not used $7.071 \mathrm{E}+01$

not used $30.000 \mathrm{E}+00^{3}$

not used $30.000 E+00^{3}$

not used $30.000 E+00$ ?

not used $30.000 E+003$

not used $0.000 \mathrm{E}+00$ ?

' not used $0.000 E+00$ '

3 not used $30.000 E+003$

3 not used $30.000 E+003$

not used $30.000 E+003$

not used $* 0.000 \mathrm{E}+00$

not used $1.000 E+00$

3 not used $2.732 E-01$

not used $: 0.000 E+00$

not used $10.000 E+00$

not used $30.000 E+00$

not used $30.000 E+00$ '

3 not used, $0.000 E+00$

3 not used $\$ 0,000 E+00$

3 not used $\$ 0.000 E+00$

not used $0.000 \mathrm{E}+00$,

' not used $30.000 \mathrm{E}+00$,

not used $0.000 E+00$

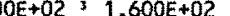

$2.700 \mathrm{E}+00,1.400 \mathrm{E}+01$

$1.000 E+02 ; 9.200 E+01$

$3.600 E+01 \times 6.300 E+01$

$31.970 \mathrm{E}+01 \times 5.400 \mathrm{E}+00$

$9.000 \mathrm{E}-01$ I $9.000 \mathrm{E}-01$

$7.300 \mathrm{E}+0133.650 \mathrm{E}+01$

$7.300 E+02=5.100 E+02$

$1.000 E+00>1.000 E+00$

not used ? $1,000 E+00$

$1.000 E+0031.000 E+00$.

$1.000 E+00^{3} 1.000 E+00$

$5.000 E-01 ; 5.000 E-01$

$3-1 \quad 3-1$ ?

$\begin{array}{lll}3-1 & 3-1 & 3 \\ 3-1 & 3-1\end{array}$

$3-1$

$36.800 \mathrm{E}+0136.800 \mathrm{E}+01$

$5.500 E+01,5.500 E+01$

$5.000 E+01 ; 5.000 E+01$

$1.600 \mathrm{E}+02 \times 1.600 \mathrm{E}+02$

3 5.000E-01, 5.000E-013
RAD SHAPE (1)

1 RAD_SHAPE( 2)

RAD SHAPE( 3 )

RAD SHAPE ( 4 )

3 RAD_SHAPE( 5 )

- RAD SHAPE 6 )

- RAD SHAPE 7 )

- RAD SHAPE ( 8 )

- RAD SHAPE( 9)

- RAD_SHAPE (10)

$\checkmark$ RAD_SHAPE (11)

RAD SHAPE (12)

- fracar 1)

- fraca( 2)

- FRACA (3)

- FRACA (4)

- Fracal 5)

- FRACA (6)

FRACA( 7)

- FRACA (8)

3 FRACA (9)

3 FRACA(10)

3 FRACA (11)

OIET(1)

3 DIET(2)

DIET(3)

3 DIET(4)

- DIET(5)

3 DIET(6)

s SOIL

DWI

3 FDW

3 FHHW

3 FLW

3 FIRW

- FRO

3 FPLANT

- FMEAT

- FMILK

LFI5

- LFI6

L LWI5

- LUIG

, LSI 
IRESRAD, Version 6.22 Ta Limit $=0.5$ year $07 / 18 / 2005 \quad 15: 52$ Page 6 Sumary : 100-K-56 pipeline Cleanup Verification RESRAD Calculation

File: 100-K-56_Deep_Zone.RAD Site-Specific Parameter Sumary (continued)

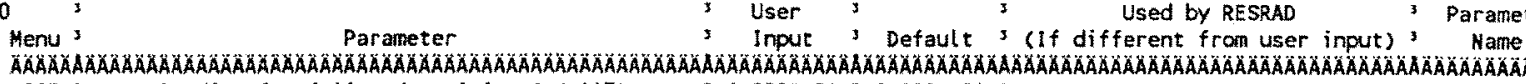
R019? Mass loading for foliar deposition $\left(\mathrm{g} / \mathrm{m}^{* * 3}\right) ; 1.000 \mathrm{E}-04,1.000 \mathrm{E}-04 ; \%$ R019 3 Depth of soil mixing layer $(\mathrm{m})$ 3 $1.500 \mathrm{E}-0131.500 \mathrm{E}-01$, R019' Depth of roots (m)

R019 Drinking water fraction fron ground water R0193 Household water fraction from ground water $\$ 9.000 \mathrm{E}-0139.000 \mathrm{E}-013$ $=1.000 \mathrm{E}+00 * 1.000 \mathrm{E}+00$ : 3 not used $1.000 E+00$ ? $=1.000 E+00 ; 1.000 E+003$

R019 I Irrigation fraction from ground water $31.000 E+00 ; 1.000 E+00 ;$

$R 19 \mathrm{~B}$ ' Wet weight crop yield for Non-Leafy $\left(\mathrm{kg} / \mathrm{m}^{* \star 2}\right)$

$R 19 B$ Wet weight crop yield for Leafy $\left(\mathrm{kg} / \mathrm{m}^{* * 2}\right.$

$R 19 B$ I Wet weight crop yield for Fodder $\left(\mathrm{kg} / \mathrm{m}^{* *} \mathrm{2}\right)$

R19B : Growing Season for Non-Leafy (years)

3.000E-01,7.000E-01; $31.500 E+00 * 1.500 E+00$ ?

$31.100 \mathrm{E}+0031.100 \mathrm{E}+00$ ?

$31.700 \mathrm{E}-0131.700 \mathrm{E}-013$

3. $2.500 \mathrm{E}-01: 2.500 \mathrm{E}-01$;

3. $8.000 \mathrm{E}-02 \times 8.000 \mathrm{E}-02$ :

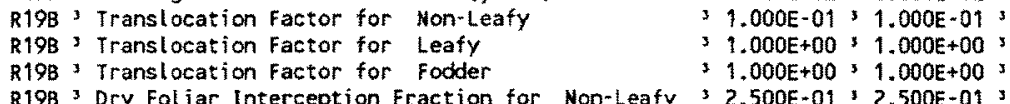

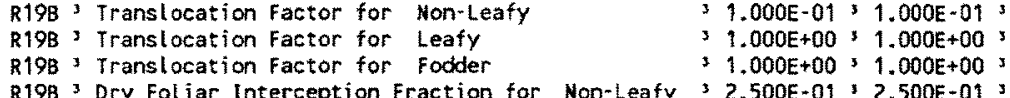

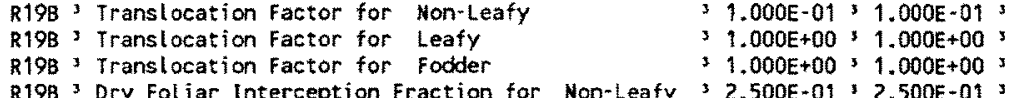

R19B 3 Dry Foliar Interception Fraction for Non-Leafy, $2.500 E-01: 2.500 E-01$,
R19B 3 Dry Foliar Interception Fraction for Leafy $2.500 E-01 ; 2.500 E-01$,

$2.500 \mathrm{E}-01 ; 2.500 \mathrm{E}-01$;

R.9. Dry Foliar Interception Fraction for Fodder, 2.500e-01,3.500E-0,

R19B 3 Wet Follar Interception Fraction for Leafy, $2.500 E-0132.500 E-01$,

$\mathrm{R} 19 \mathrm{~B}$ * Wet Foliar Interception Fraction for Fodder $32.500 \mathrm{E}-0132.500 \mathrm{E}-013$

R19B * Weathering Removal Constant for Vegetation

3

' 2.000E+01'2.000E+01?

3

, not used $2.000 E-051$

3 not used 3.000 E 02 '

3 not used 32.000 E-02,

' not used $39.800 E-01$ *

' not used 33.000 E 01 '

' not used 3 7.000E-07's

, not used $\$ 1.000$ E-10'

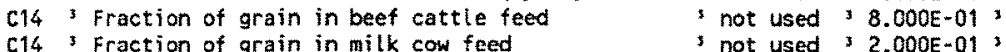

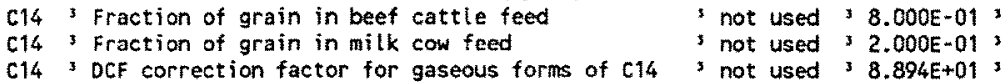

3

STOR 3 Storage times of contaminated foodstuffs (days): *

STOR ${ }^{3} \quad$ Eruits, non-leafy vegetables, and grain

STOR * Leafy vegetables

STOR : Milk

STOR Meat and poultry

STOR Fish

STOR * Crustacea and nollusks

STOR $\rightarrow$ Well water

STOR: SUrface water

STOR " Livestock fodder

R021 Thickness of building foundation (n)

R021 Bulk density of building foundation $(9 / \mathrm{cm} * 3$ )

R0213 Total porosity of the cover material

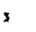

$1.400 E+0131.400 E+01$

$>1.000 E+00 \div 1.000 E+00$ ?

$31.000 E+00 ; 1.000 E+00$ '

$2.000 E+01^{3} 2.000 E+01$,

$3.000 E+00^{3} 7.000 E+003$

$37.000 E^{3}+00^{3} 7.000 E+003$

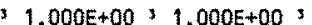

$31.000 \mathrm{E}+0031.000 \mathrm{E}+00$,

$4.500 \mathrm{E}+0134.500 \mathrm{E}+01:$

not used $31.500 \mathrm{E}-01$ '

* not used $32.400 E+00^{3}$

$x$ not used 4.000 E-01:

...

... 3 DROOT

... 3 FGWDN

FGWHK

₹ FGWL

-.- 3 FGWIR

$\ldots Y Y(1)$

$Y V(2)$

YV(3)

$\cdots$ TE(1)

$\ldots \quad$ TE(3)

s TIV(1)

... 3 TIV(2)

... 3 TIV $(3)$

$\ldots+3$ RDRY(1)

3 RDRY (2)

3 RORY (3)

‥ 3 RWET(1)

...

...

W. I CILWTR

...

CAIR

-..

REVSN

*. 3 AVFG4

-.. 3 AVFG5

...

I

STOR T(1)

$\cdots$

‥ $\quad$ STOR T(2)

...

, STOR T(4)

, STORT(5)

yTOR

... Y STOR TCT

* STORTT(8)

... STOR_T(9)

,... FLOOR1

...

S TPCY 
CVP-2005-00006

Rev. 0

IRESRAD, Version 6.22 Ta Limit $=0.5$ year $\quad 07 / 18 / 2005 \quad 15: 52 \quad$ Page 7

Sumary : 100-K-56 Pipeline Cleanup Verification RESRAD Calculation

File : 100-K-56_peep_zone.RAD Site-specific Parameter sumary (continued)

\section{MenU 3}

Parameter

I User

(Defate

Used by RESRAD

Used by RESRAD I Parameter

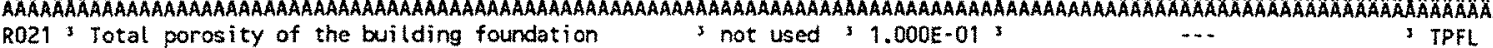

R021 Volumetric water content of the cover material s not used $35.000 \mathrm{E}-02$.

R021' Volumetric water content of the foundation

R021 Diffusion coefficient for radon gas $(\mathrm{m} / \mathrm{sec})$ :

R021 in cover material

RO21 3 in foundation material

R021 3 in contaninated zone soil

R021 Radon vertical dimension of mixing (m)

R021 Average bullding air exchange rate (1/hr)

R021 Height of the building (room) (m)

R021 Building interior area factor

R021 Bullding depth below ground surface (m)

not used $3,000 \mathrm{E}+02$

1

nat used $2.000 \mathrm{E}-06$ :

not used 3,000E-07

3 not used 3 2.000E-06

3 not used $32.000 E+00$

7 not used' 5.000E-01'

not used $2.500 E+00$

3 not used $30.000 E+00$

s not used $1-1.000 E+00$,

not used 2.500 -01'

3 not used 31.500 E-01'

R021. Emanating power of $R_{n}-220$ gas

5

IITL Number of graphical time points

IITL Maximum number of integration points for dose

IIIL " Maximum number of integration points for risk

32 ;

$\begin{array}{rlll}32 & 3 & \cdots & 3\end{array}$

(2)

SDIFCV

-. 3 DIFFL

...

a. HMIX

3 REXG

3 HRM

$+\cdots$

a..

B.. EMANA (1)

.. 3 EMANAC?

-.. $\quad 3$ NPTS

$\cdots$

, LYMAX

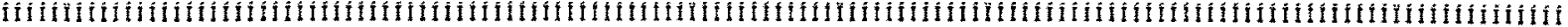

Sumary of Pathway Selections

Pathway, User Selection

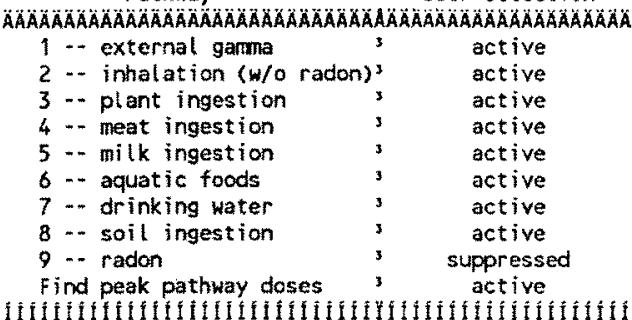


CVP-2005-00006

Rev. 0

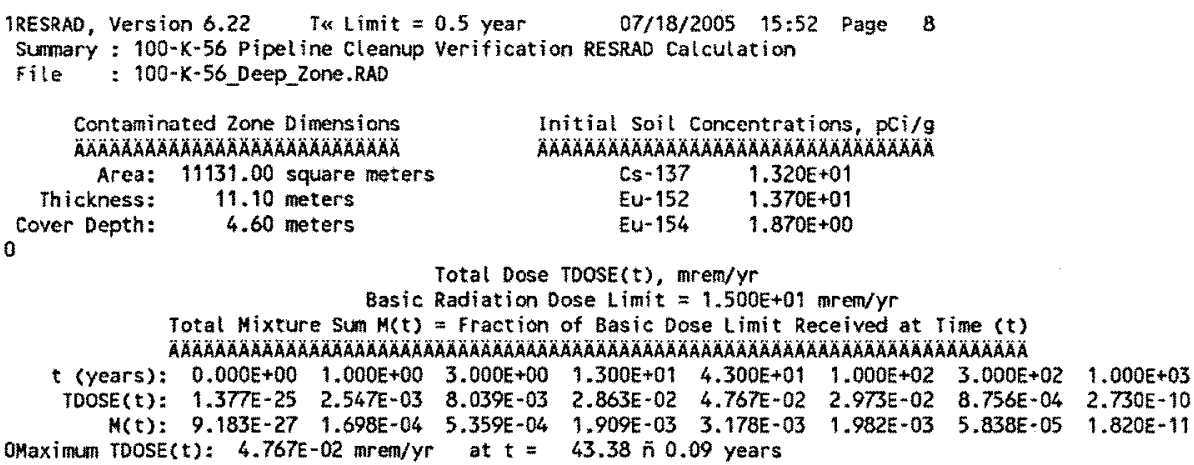


CVP-2005-00006

Rev. 0

\section{CALCULATION BRIEF EXCERPTS}

C-51 
CVP-2005-00006

Rev. 0

\section{DISCLAIMER FOR CALCULATIONS}

The attached calculations have been generated for a specific purpose and task. Use of these calculations by persons who do not have access to all pertinent facts may lead to incorrect conclusions and/or results. Before applying these calculations to your work, the underlying basis, rationale, and other pertinent information relevant to these calculations must be thoroughly reviewed with appropriate Washington Closure Hanford officials or other authorized personnel. Washington Closure Hanford is not responsible for the use of a calculation not under its direct control. 


\section{CALCULATION BRIEFS}

The following calculation briefs have been prepared in accordance with BHI-DE-01, Design Engineering Procedures Manual, EDPI-4.37-01, "Project Calculations," Bechtel Hanford, Inc., Richland, Washington.

100-K-55 Pipeline and Overburden Variance Calculation, Calculation No. 0100K-CA-V0041, Rev. 0, Bechtel Hanford, Inc., Richland, Washington.

100-KW-55 Pipelines Shallow, Deep, and Overburden Zone Sampling Plan, Calculation No. 0100K-CA-V0039, Rev. 0, Bechtel Hanford, Inc., Richland, Washington.

100-K-55:1 Pipeline Cleanup Verification 95\% UCL Calculation, Calculation No. 0100K-CA-V0045, Rev. 0, Bechtel Hanford, Inc., Richland, Washington.

100-K-55:1 Pipeline RESRAD Calculation, Calculation No. 0100K-CA-V0046, Rev. 0, Bechtel Hanford, Inc., Richland, Washington.

100-K-55:1 Comparison to Drinking Water Standards (MCL) Calculation Brief, Calculation No. 0100K-CA-V0047, Rev. 0, Bechtel Hanford, Inc., Richland, Washington.

100-K-56:1 Pipeline Variance Calculation, Calculation No. 0100K-CA-V0052, Rev. 0, Bechtel Hanford, Inc., Richland, Washington.

100-K-56:1 Pipelines Shallow and Deep Zone Sampling Plan, Calculation No. 0100K-CA-V0053, Rev. 0, Bechtel Hanford, Inc., Richland, Washington.

100-K-56:1 Pipeline Cleanup Verification 95\% UCL Calculation, Calculation No. 0100K-CA-V0049, Rev. 0, Bechtel Hanford, Inc., Richland, Washington.

100-K-56:1 Pipeline RESRAD Calculation, Calculation No. 0100K-CA-V0050, Rev. 0, Bechtel Hanford, Inc., Richland, Washington.

100-K-56:1 Comparison to Drinking Water Standards (MCL) Calculation, Calculation No. 0100K-CA-V0051, Rev. 0, Bechtel Hanford, Inc., Richland, Washington.

116-KW-4 and 116-KE-5 Heat Recovery Stations Hazard Quotient and Carcinogenic Risk Calculations, Calculation No. 0100K-CA-V0054, Rev. 0, Bechtel Hanford, Inc., Richland, Washington.

NOTE: The calculation briefs referenced in this appendix are kept in the active Washington Closure Hanford project files and are available upon request. When the project is completed, the files will be stored in a U.S. Department of Energy, Richland Operations Office repository. Only excerpts of the calculation briefs are included in this appendix. 
CVP-2005-00006

Rev. 0

C-54 


\section{CALCULATION COVER SHEET}

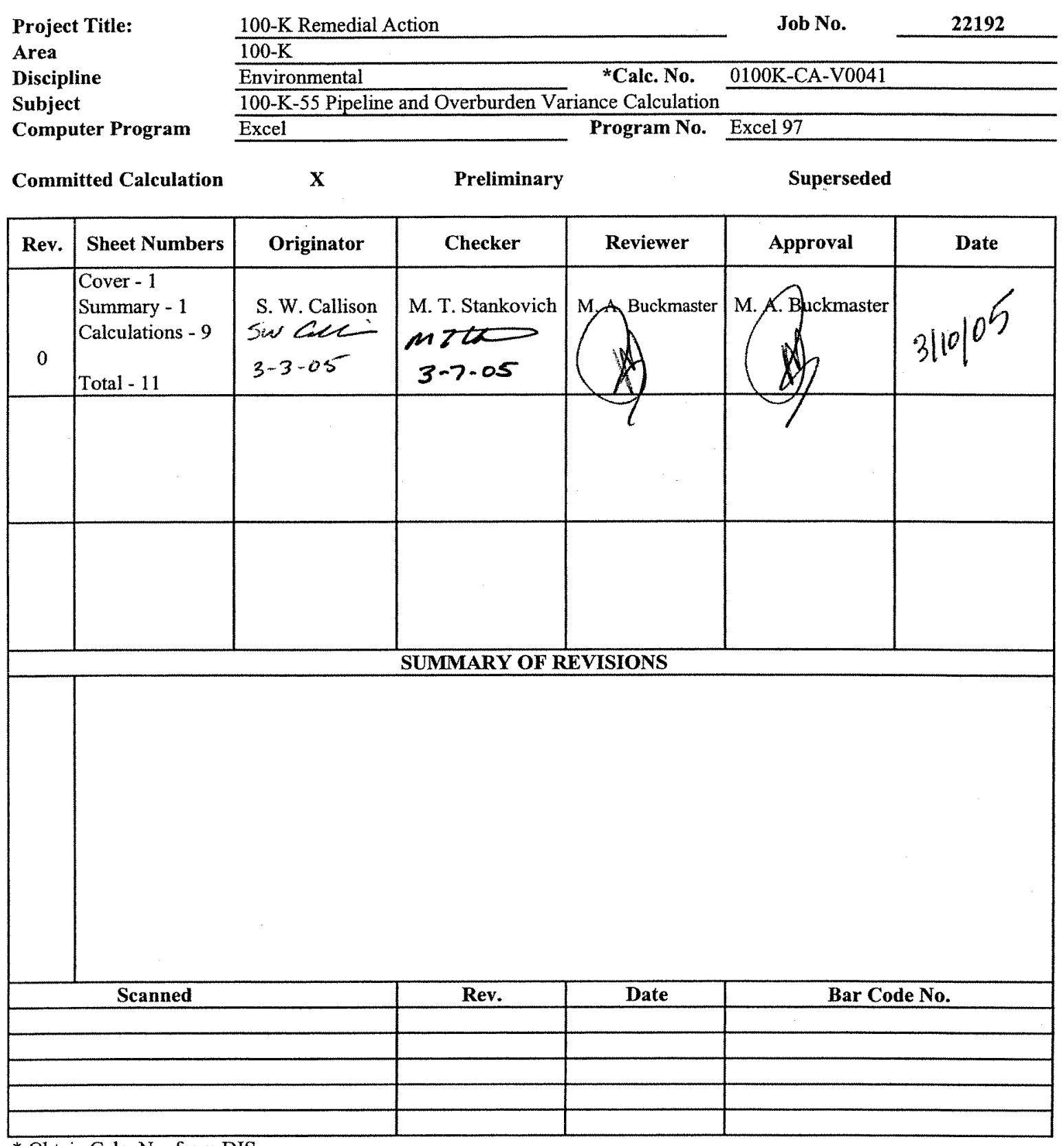

\footnotetext{
Obtain Calc. No. from DIS.
} 
CVP-2005-00006

Rev. 0

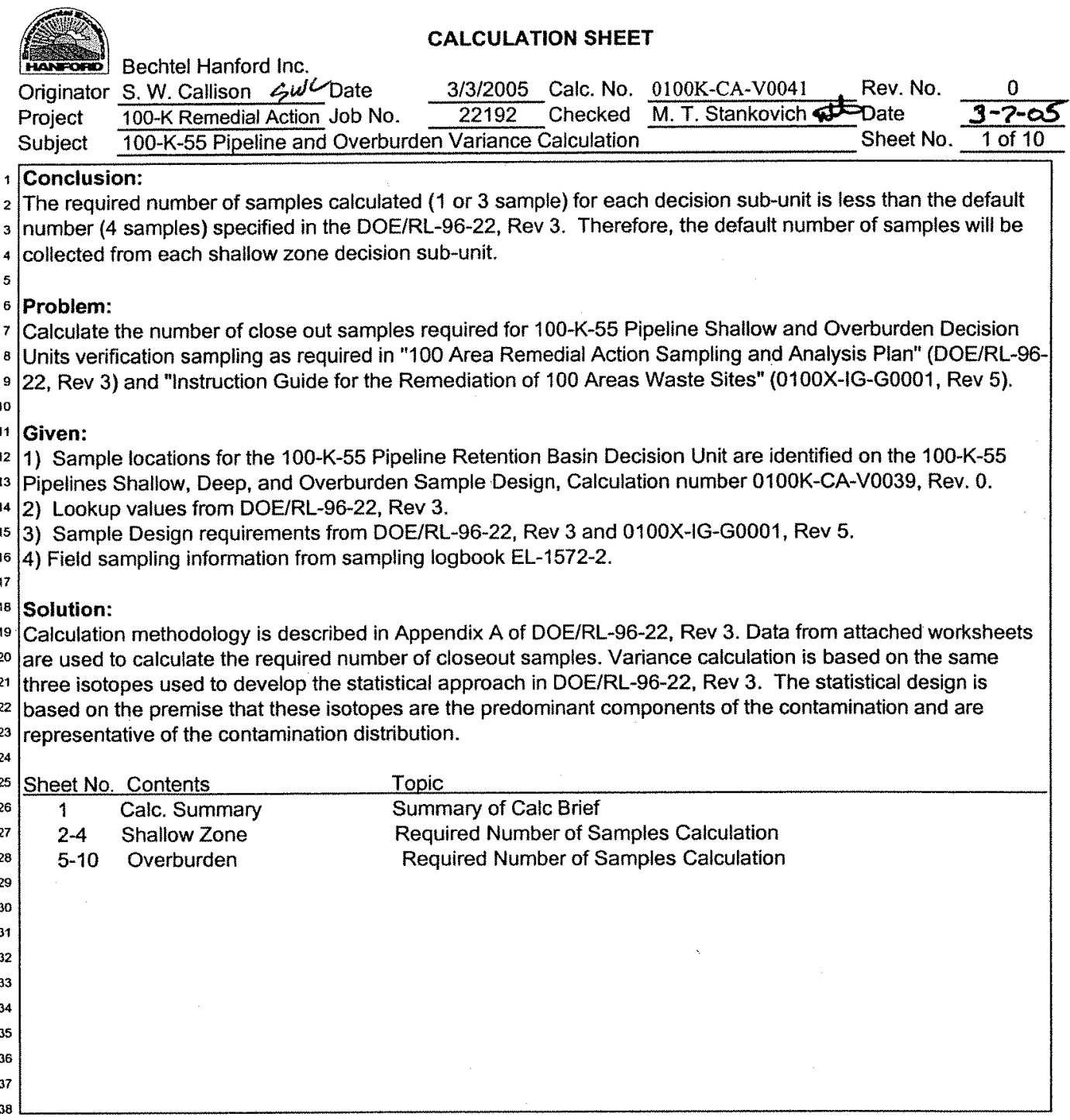




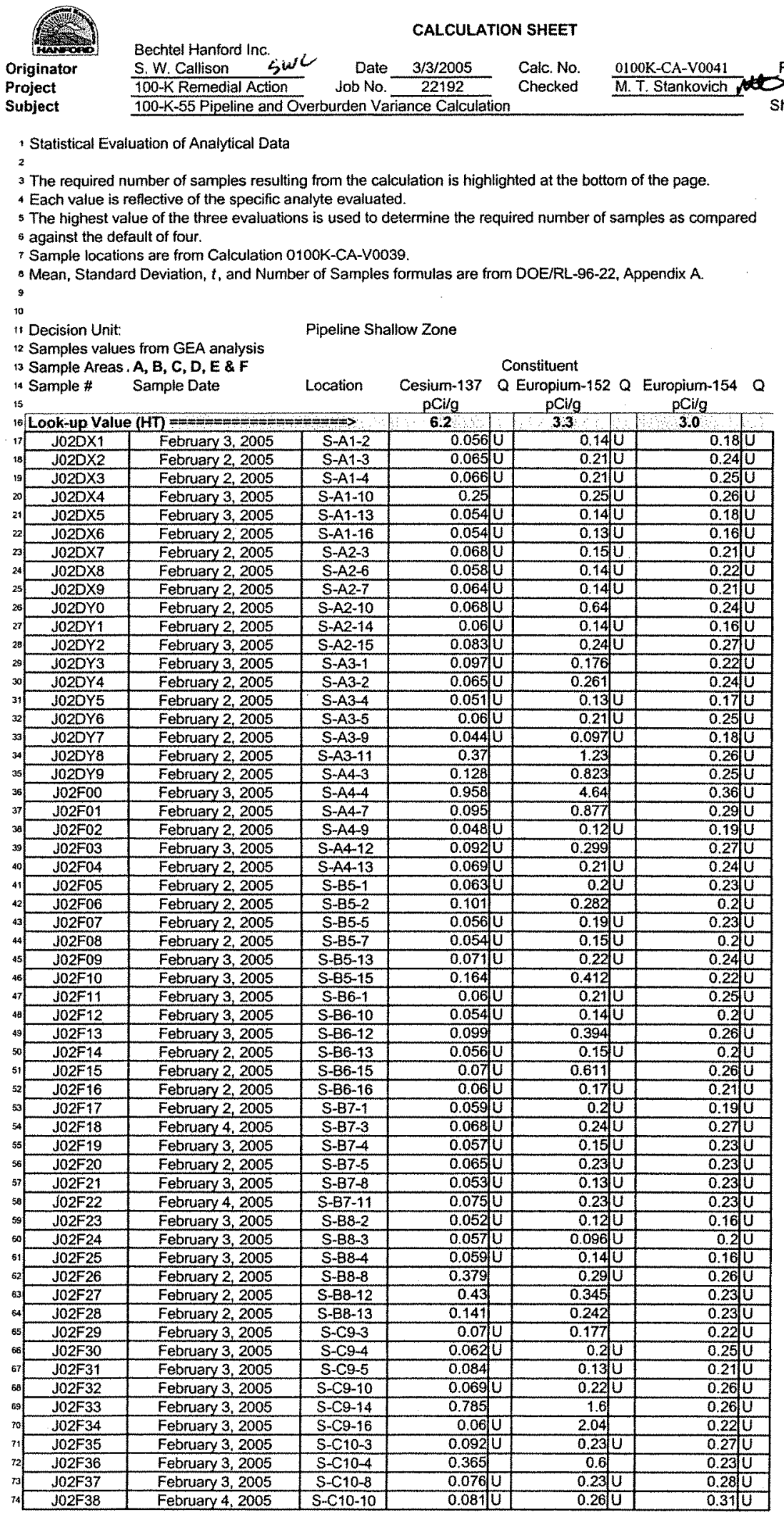


CVP-2005-00006

Rev. 0

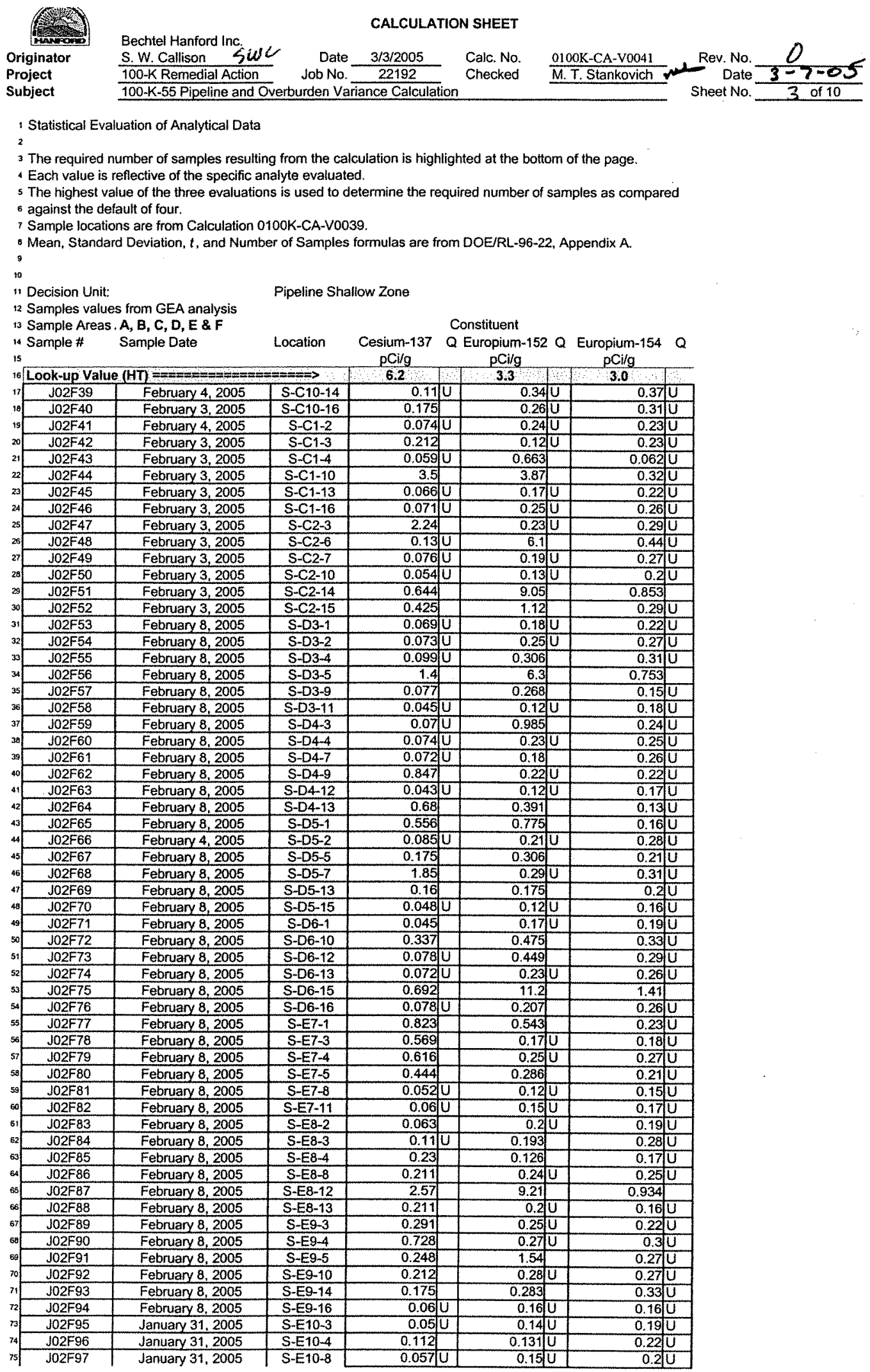

Shallow Zone 


\section{CALCULATION SHEET}

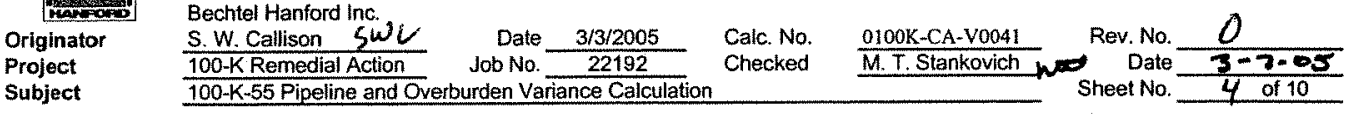

I Statistical Evaluation of Analytical Data

3 The required number of samples resulting from the calculation is highlighted at the bottom of the page.

- Each value is reflective of the specific analyte evaluated.

5 The highest value of the three evaluations is used to determine the required number of samples as compared

6 against the default of four.

7 Sample locations are from Calculation 0100K-CA-V0039.

- Mean, Standard Deviation, $t$, and Number of Samples formulas are from DOE/RL-96-22, Appendix A.

11 Decision Unit:

2 Samples values from GEA analysis

is Sample Areas. A, B, C, D, E \& F

14 Sample \# Sample Date

Pipeline Shallow Zone

16 Look-up Valiue

Constituent

Location Cesium-137 Q Europium-152 Q Europium-154 Q

\begin{tabular}{|c|c|c|c|c|c|c|c|c|}
\hline \multirow{2}{*}{\multicolumn{3}{|c|}{ Look-up Value $(\mathrm{HT})=-\mathrm{C}=\mathrm{C}=\mathrm{C}$}} & \multirow{2}{*}{\multicolumn{2}{|c|}{6.2}} & \multirow{2}{*}{\multicolumn{2}{|c|}{3,3}} & \\
\hline & & & & & & & 3.0 & \\
\hline J02F98 & January 31,2005 & S-E10-10 & 0.047 & & 0.11 & U & 0.16 & U \\
\hline $\mathrm{J} 02 \mathrm{Fg9}$ & January 31,2005 & S-E10-14 & 0.049 & U & 0.12 & $\mathrm{U}$ & 0.18 & U \\
\hline $\mathrm{J} 02 \mathrm{FBO}$ & January 31,2005 & $S+E 10 \cdot 16$ & 0.052 & U & 0.13 & U & 0.19 & U \\
\hline $\mathrm{J} 02 \mathrm{FB1}$ & January 27,2005 & S-F1-2 & 0.059 & $\mathrm{U}$ & 0.13 & $\mathrm{U}$ & 0.17 & $\mathrm{U}$ \\
\hline $\mathrm{J} 02 \mathrm{FB} 2$ & January 27,2005 & $\mathrm{~S}-\mathrm{F} 1-3$ & 0.061 & $\mathrm{U}$ & 0.15 & $\mathrm{U}$ & 0.2 & $\mathrm{U}$ \\
\hline $\mathrm{J} 02 \mathrm{FB} 3$ & January 27,2005 & S-F1-4 & 0.041 & $\mathrm{U}$ & 0.11 & IU & 0.15 & $\mathrm{U}$ \\
\hline $\mathrm{J} 02 \mathrm{FB} 4$ & January 27,2005 & S-F1-10 & 0.043 & $\mathrm{U}$ & 0.12 & 40 & 0.18 & U \\
\hline $\mathrm{J} 02 \mathrm{FB} 5$ & January 27,2005 & $S-F 1-13$ & 0.062 & U & 0.14 & 1u & 0.24 & U \\
\hline J02FB6 & January 27.2005 & S-F1-16 & 0.054 & $\mathrm{U}$ & 0.18 & U & 0.2 & $\mathrm{U}$ \\
\hline J02FB7 & January 27,2005 & $S-F 2-3$ & 0.052 & $\mathrm{U}$ & 0.15 & एu & 0.18 & $\mathrm{U}$ \\
\hline $\mathrm{J} 02 \mathrm{FB} 8$ & January 27,2005 & S-F2-6 & 0.046 & $\mathrm{U}$ & 0.11 & U & 0.16 & U \\
\hline J02FB9 & January 27,2005 & S-F2-7 & 0.052 & $\mathrm{U}$ & 0.17 & U & 0.2 & 10 \\
\hline $\mathrm{J} 02 \mathrm{FCO}$ & January 27,2005 & S.F2-10 & 0.049 & $\mathbf{U}$ & 0.15 & U & 0.19 & $\mathrm{U}$ \\
\hline $\mathrm{J} 02 \mathrm{FC1}$ & January 27,2005 & S-F2-14 & 0.039 & $\mathrm{U}$ & 0.11 & U & 0.18 & U \\
\hline J02FC2 & January 27,2005 & S-F2-15 & 0.049 & $\mathrm{u}$ & 0.16 & U & 0.18 & U \\
\hline $102 \mathrm{FC} 3$ & January 27,2005 & S-F3-1 & 0.07 & U & 0.16 & $\mathrm{U}$ & 0.23 & U \\
\hline J02FC4 & January 27,2005 & S-F3-2 & 0.049 & $\mathrm{U}$ & 0.14 & $\mathrm{U}$ & 0.2 & $\mathrm{U}$ \\
\hline J02FC5 & January 27,2005 & S-F3-4 & 0.057 & U & 0.18 & $\mathrm{U}$ & 0.19 & $\mathrm{U}$ \\
\hline $\mathrm{J} 02 \mathrm{FC6}$ & January 27,2005 & S-F3-5 & 0.053 & U & 0.15 & SU & 0.25 & $\mathrm{U}$ \\
\hline $102 \mathrm{FC7}$ & January 27,2005 & S-F $3-9$ & 0.049 & U & 0.12 & ए & 0.17 & $\mathrm{U}$ \\
\hline $\mathrm{J} 02 \mathrm{FCB}$ & January 27,2005 & $S-F 3-11$ & 0.049 & U & 0.16 & E & 0.18 & $\mathrm{U}$ \\
\hline $\mathrm{J} 02 \mathrm{FC} 9$ & January 27,2005 & S.F4-3 & 0.057 & $\mathrm{U}$ & 0.15 & bु & 0.16 & $\mathrm{U}$ \\
\hline J02FDO & January 27,2005 & S-F4-4 & 0.049 & $\mathrm{U}$ & 0.12 & U & 0.17 & $\mathrm{U}$ \\
\hline J02FD1 & January 27,2005 & S-F4-7 & 0.05 & U & 0.16 & 3u & 0.21 & U \\
\hline $\mathrm{J} 02 \mathrm{FD} 2$ & January 27,2005 & S-F4-9 & 0.051 & U & 0.13 & 3 & 0.16 & है \\
\hline $\mathrm{J} 02 \mathrm{FD} 3$ & January 27,2005 & $S-F 4-12$ & 0.037 & U & 0.11 & IU & 0.13 & $3 \mathrm{U}$ \\
\hline $102 \mathrm{FD} 4$ & January 27,2005 & $S-F-13$ & 0.046 & U & 0.15 & $\mathrm{U}$ & 0.18 & $\mathrm{U}$ \\
\hline \multicolumn{3}{|c|}{ 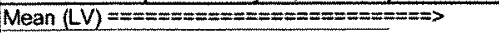 } & 0.22 & & 0.61 & & 0.26 & \\
\hline \multicolumn{3}{|c|}{ Standard Deviation $(S)============\Rightarrow$} & 0.46 & & 1.62 & & 0.15 & \\
\hline \multicolumn{3}{|c|}{ 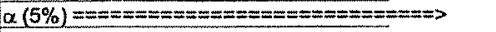 } & 1.645 & & 1.645 & & 1.645 & \\
\hline \multirow{2}{*}{\multicolumn{3}{|c|}{ 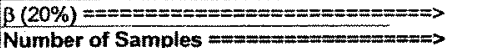 }} & 0.842 & & 0.842 & & 0.842 & \\
\hline & & & 1 & & 3 & & 1 & \\
\hline
\end{tabular}


CVP-2005-00006

Rev. 0

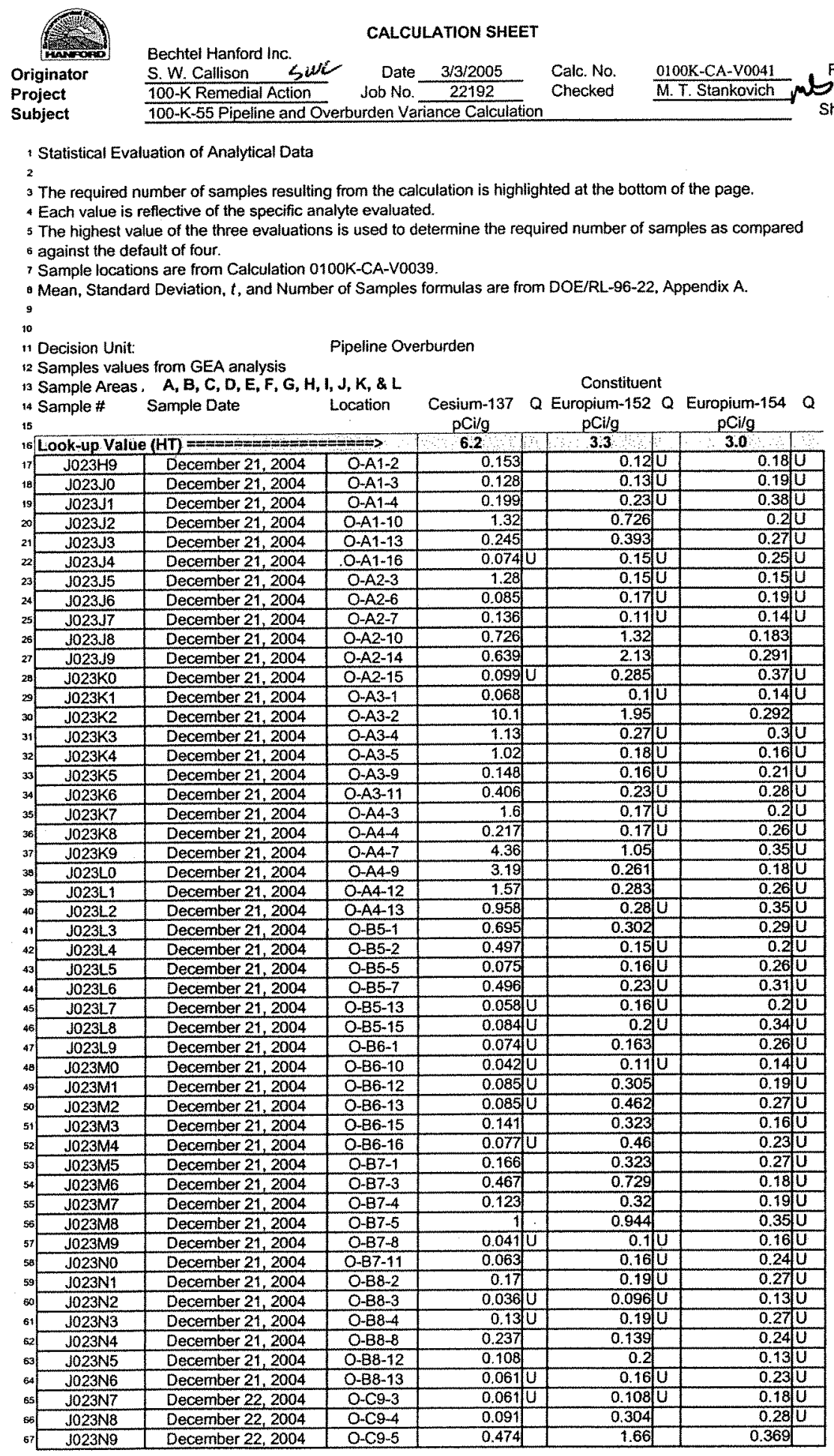




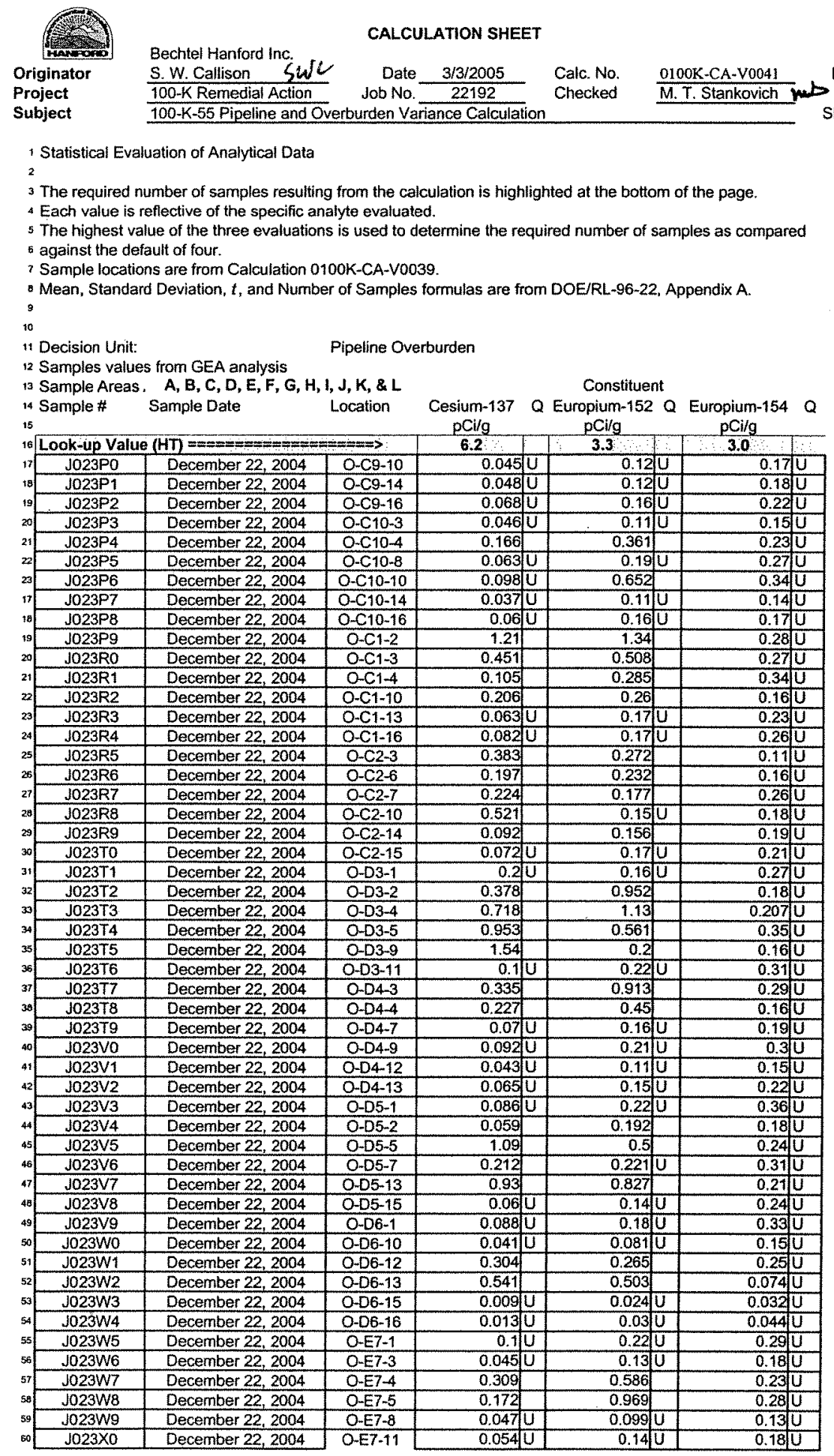


Bechtel Hanford inc. S.W. Callison Sut

CALCULATION SHEET

Subject

$100-K-55$ Pipeline and Overburden $\frac{22192}{10}$

$\begin{array}{ll}\text { Calc. No. } & \text { 0100K-CA-V0041 } \\ \text { Checked } & \text { M.T.Stankovich }\end{array}$

Rev. No.

Dheet No. $\frac{3-7.03}{7 \text { of } 10}$

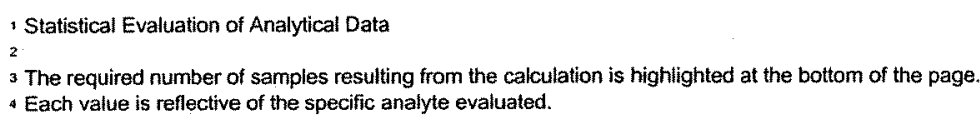

- Statistical Evaluation of Analytical Data

3 The required number of samples resulting from the calculation is highlighted at the bottom of the page.

4 Each value is reflective of the specific analyte evaluated.

5 The highest value of the three evaluations is used to determine the required number of samples as compared

$\checkmark$ against the default of four.

1 Sample locations are from Calculation 0100K-CA-V0039.

a Mean, Standard Deviation, $t$, and Number of Samples formulas are from DOE/RL-96-22, Appendix A.

9

11 Decision Unit

12 Samples values from GEA analysis

is Sample Areas. A, B, C, D, E, F, G, H, I, J, K, \& L

Pipeline Overburden

Cesium-137 Q Europium-152 Q Europium-154 Q

15 Look-up Value (HT) $=\Rightarrow$

$\mathrm{pCi} / \mathrm{g}$

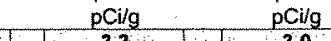

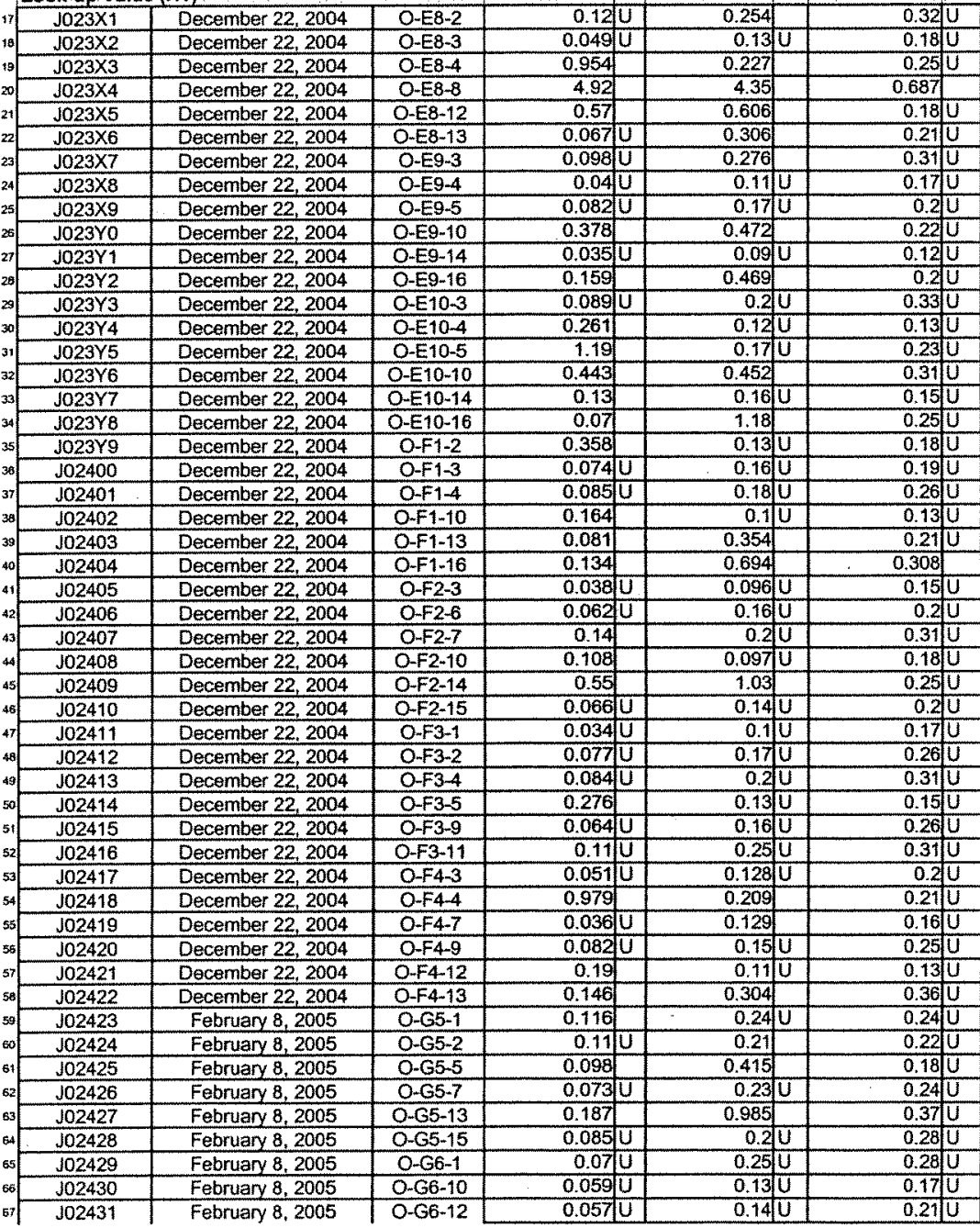


CVP-2005-00006

Rev. 0

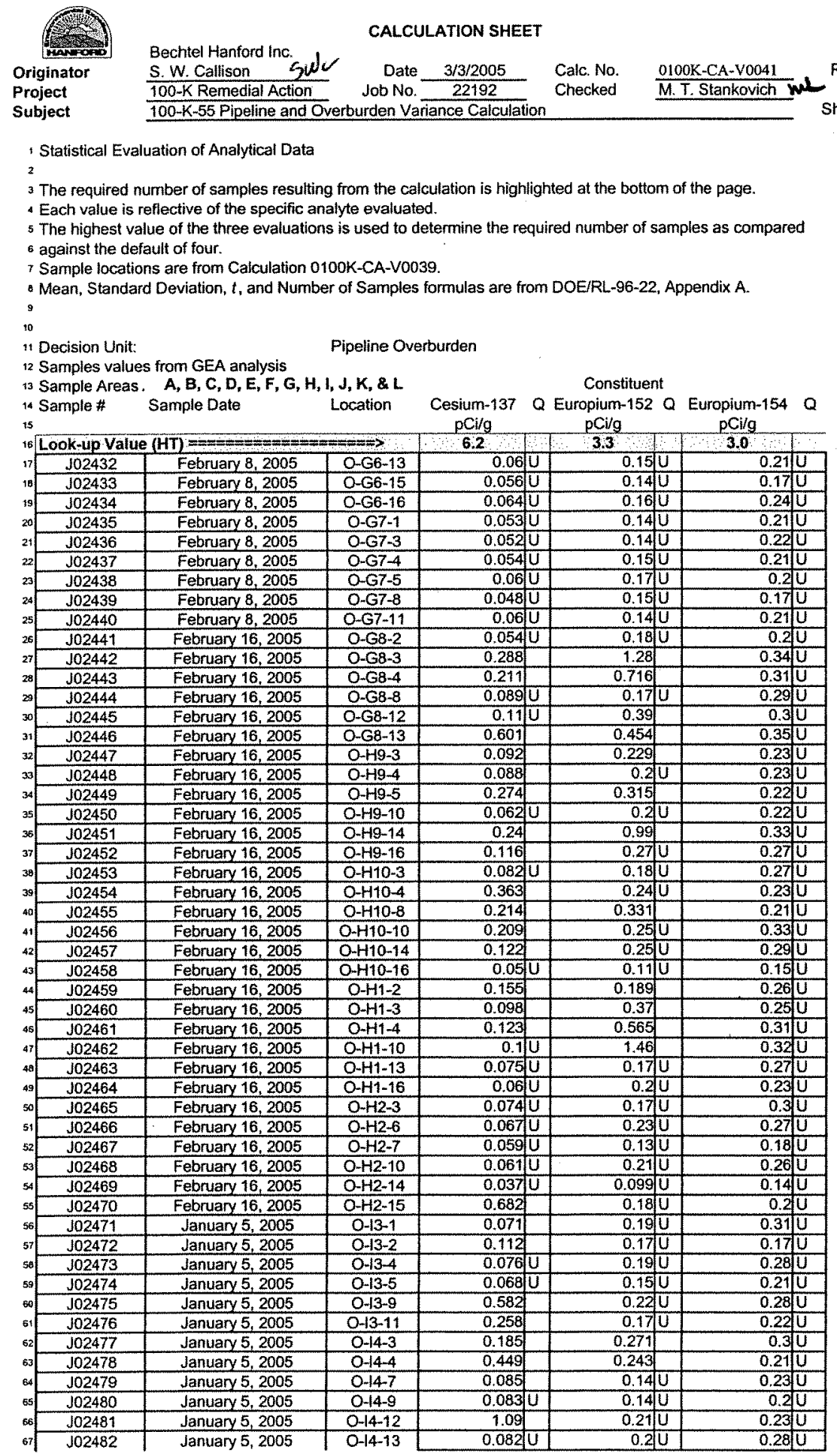


CVP-2005-00006

Rev. 0

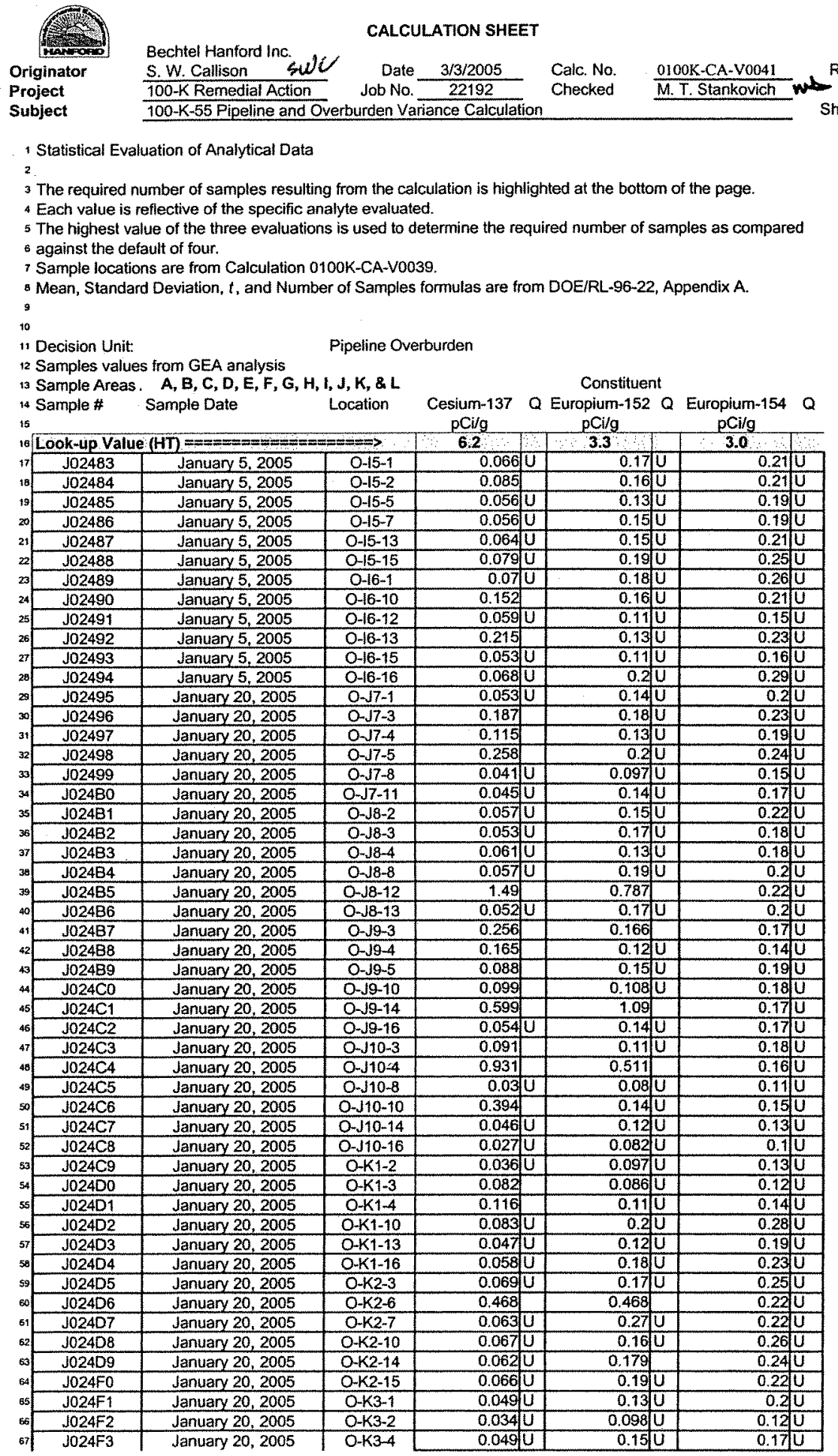


CVP-2005-00006

Rev. 0

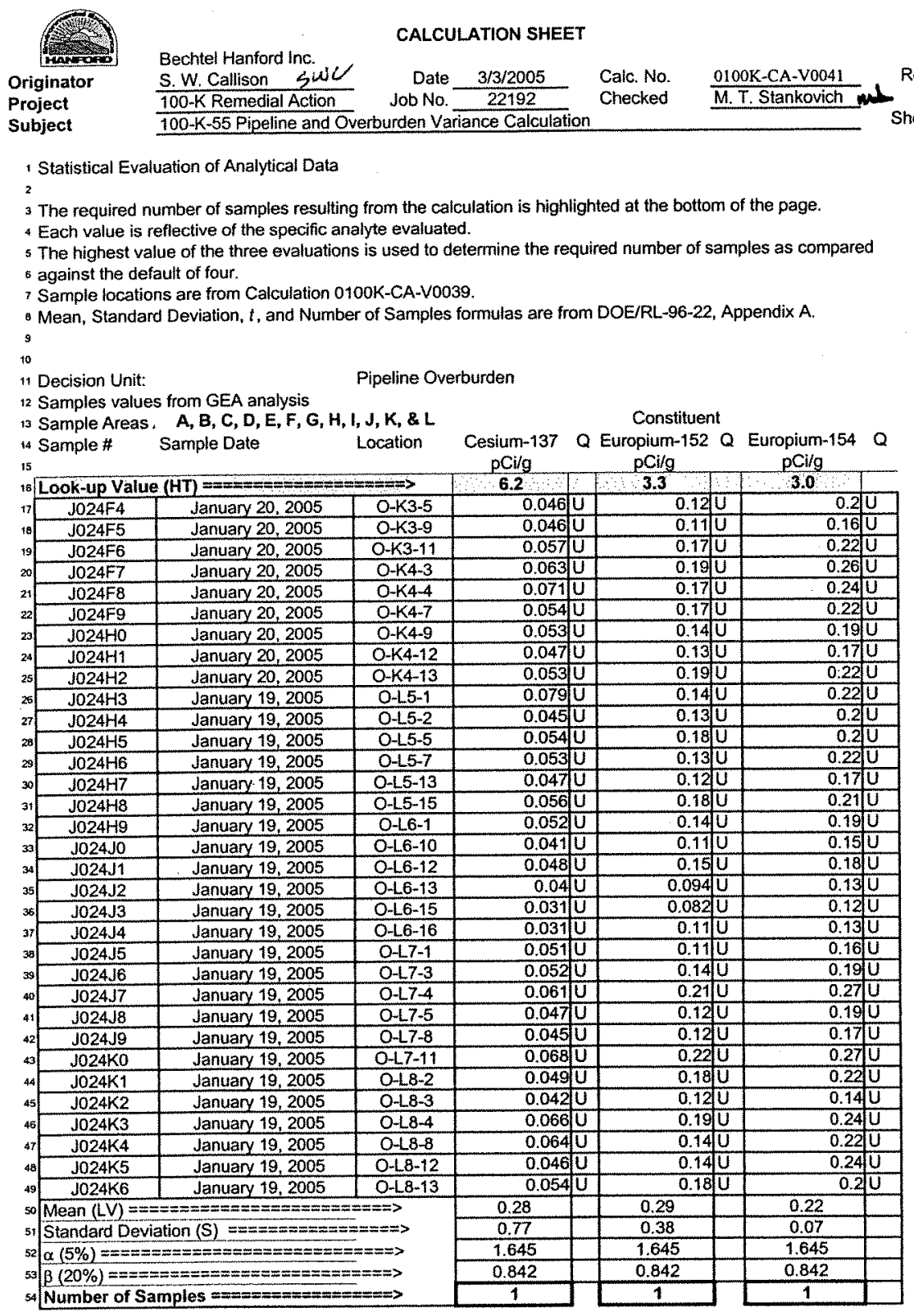


CVP-2005-00006

Rev. 0

C-66 
CVP-2005-00006

Rev. 0

\section{CALCULATION COVER SHEET}

(Sample Design, Verification, or Waste Characterization Calculation)

Project Title:

Area

Discipline

Subject

Computer Program
100-KW-55 Pipelines Sample Design 100-K

Environmental Engineering

Job No.

22192

Calc. No. 0100K-CA-V0039

Exce

Program No. Excel 97

The attached calculations have been generated to document compliance with established cleanup levels. These documents should be used in conjuction with other relevent documents in the administrative record.

Committed Calculation

X Preliminary

Superseded

\begin{tabular}{|c|c|c|c|c|c|c|}
\hline Rev. & Sheet Numbers & Originator & Checker & Reviewer & Approval & Date \\
\hline 0 & $\begin{aligned} \text { Cover } & =1 \mathrm{Sht} \\
\text { Calc } & =2 \mathrm{Shts} \\
\text { Attach } 1 & =1 \mathrm{Sht} \\
\text { Attach } 2 & =1 \mathrm{Sht} \\
\text { Attach } 3 & =6 \mathrm{Shts}\end{aligned}$ & $\begin{array}{l}\text { MCey } \\
\text { G. Cruz } \\
\sqrt{28 / 05}\end{array}$ & $\begin{array}{l}\text { Cobint } \\
\text { c.A. Bentz } \\
1 / 31 / 05\end{array}$ & $\begin{array}{l}\text { M.T. } \\
\text { Stankovich } \\
21.105\end{array}$ & $\begin{array}{l}\text { Sw Cale } \\
\text { S.w. Callison } \\
2.2-05\end{array}$ & 2.2 .05 \\
\hline & Total $=11$ Shts & & & & & \\
\hline & & & & & & \\
\hline & & & & & & \\
\hline & & & & & & \\
\hline & & & & & & \\
\hline & & & & & & \\
\hline \multicolumn{7}{|c|}{ SUMMARY OF REVISIONS } \\
\hline & & & & & & \\
\hline & & & & & & \\
\hline
\end{tabular}

* Obtain Calc. No, from DIS

January 2003

DE01-437.03 
CVP-2005-00006

Rev. 0

Bechtel Hanford, Inc.

CALCULATION SHEET

Han a o

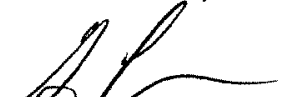

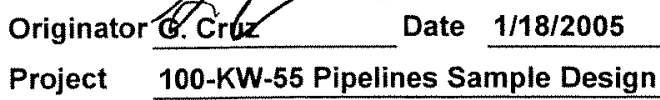

Calc. No. 0100K-CA-V0039

Rev. No. 0

Job No. 22192 Checked

Subject 100-KW-55 Pipelines Shallow, Deep, and Overburden Zone Sampling Plan

$\frac{\text { OJB }}{\text { Sheet No. } 1 \text { of } 2}$

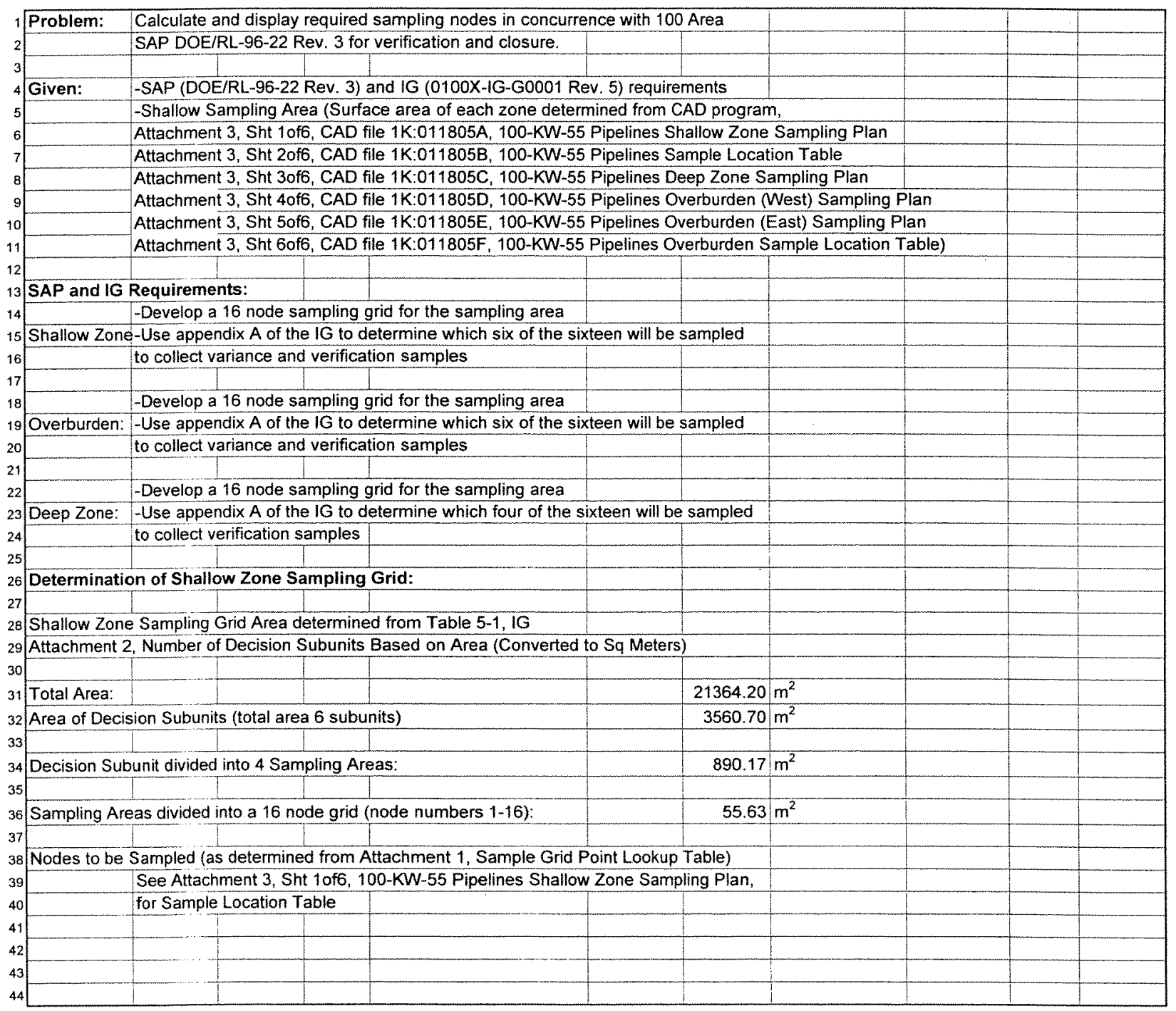


CVP-2005-00006

Rev. 0

Date $1 / 18 / 2005$

Project 100-KW-55 Pipelines Sample Design
Calc. No. 0100K-CA-V0039 Job No. 22192 Checked

Subject 100-KW-55 Pipelines Shallow, Deep, and Overburden Zone Sampling Plan
Rev. No. 0 OSB Date $/ / 3 / 05$

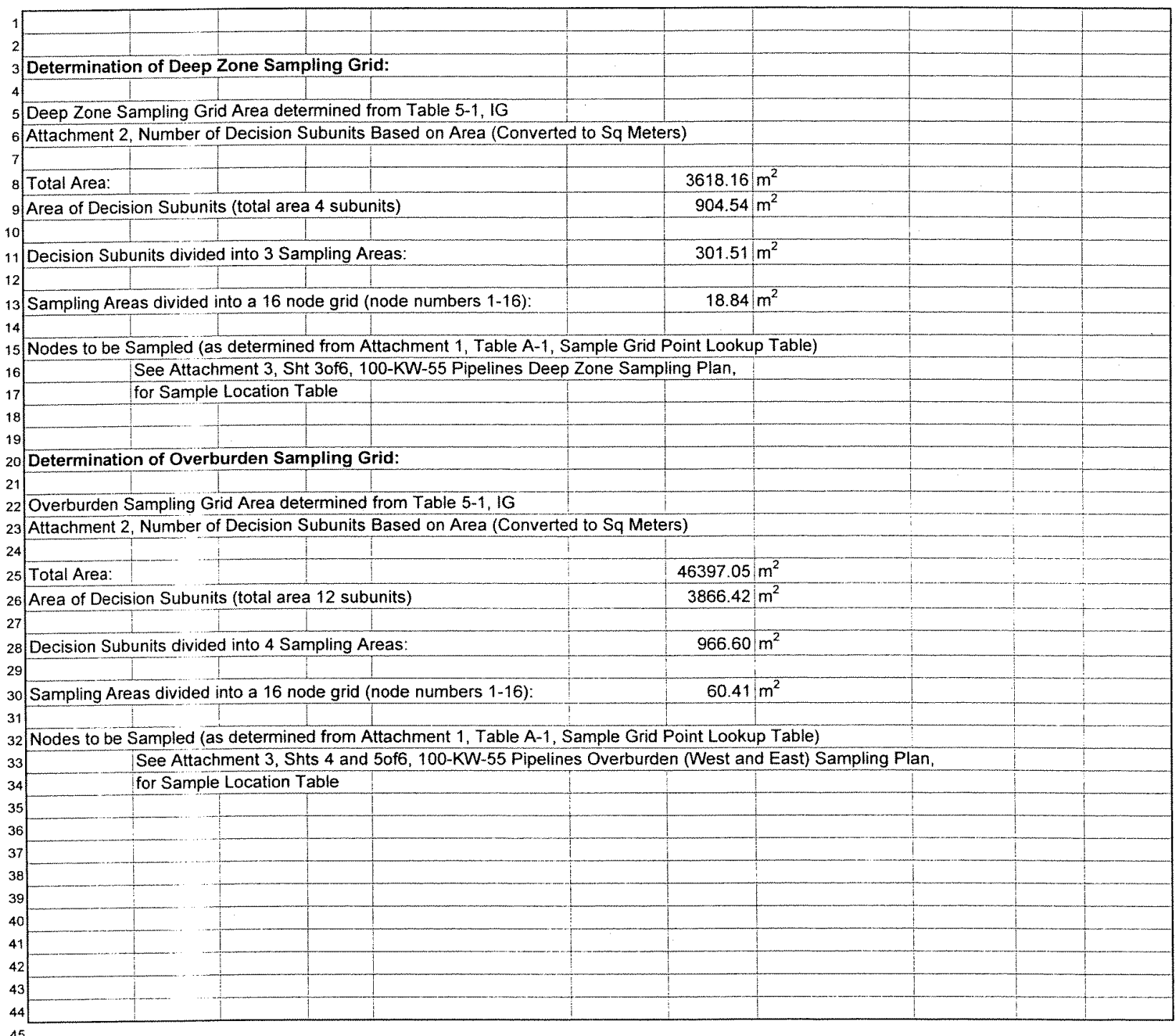


CVP-2005-00006

Rev. 0

Bechtel Hanford, Inc

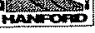

Originator

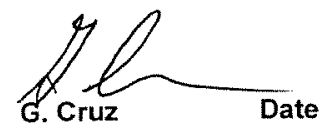

Date 1/18/2005

Calc. No.0100K-CA-V0039

Rev. No.0

Project

100-KW-55 Pipelines Sample Design Job No. 22192

Checked

cs 8 Date Sheet No 1 of 1

Subject 100-KW-55 Pipelines Shallow, Deep, and Overburden Zone Sampling Plan

\section{ATTACHMENT 1}

${ }_{3}$ Sample Grid Point Lookup Table.

\begin{tabular}{|c|c|c|c|c|c|c|c|c|c|c|}
\hline Default Plan & $\begin{array}{c}\text { Sampling } \\
\text { Area } 1\end{array}$ & $\begin{array}{c}\text { Sampling } \\
\text { Area } 2\end{array}$ & $\begin{array}{c}\text { Sampling } \\
\text { Area } 3\end{array}$ & $\begin{array}{c}\text { Sampling } \\
\text { Area } 4\end{array}$ & $\begin{array}{c}\text { Sampling } \\
\text { Area } 5\end{array}$ & $\begin{array}{c}\text { Sampling } \\
\text { Area } 6\end{array}$ & $\begin{array}{l}\text { Sampling } \\
\text { Area } 7\end{array}$ & $\begin{array}{c}\text { Sampling } \\
\text { Area } 8\end{array}$ & $\begin{array}{c}\text { Sampling } \\
\text { Area } 9\end{array}$ & $\begin{array}{c}\text { Sampling } \\
\text { Area } 10\end{array}$ \\
\hline VarianceNerification & 3 & 6 & 1 & 4 & 5 & 1 & 3 & 3 & 4 & 16 \\
\hline Variance/Nerification & 4 & 7 & 11 & 3 & 15 & 15 & 5 & 13 & 10 & 10 \\
\hline Variance/verification & 16 & 3 & 2 & 7 & 7 & 10 & 11 & 4 & 3 & 14 \\
\hline Variance/verification & 10 & 15 & 4 & 12 & 1 & 13 & 4 & 8 & 16 & 4 \\
\hline Variance & 2 & 14 & 5 & 9 & 13 & 12 & 8 & 2 & 14 & 8 \\
\hline Variance & 13 & 10 & 9 & 13 & 2 & 16 & 1 & 12 & 5 & 3 \\
\hline Not Sampling & 6 & 1 & 10 & 8 & 14 & 4 & 16 & 5 & 8 & 6 \\
\hline Not Sampling & 1 & 9 & 13 & 1 & 10 & 5 & 12 & 1 & 1 & 15 \\
\hline Not Sampling & 9 & 12 & 7 & 5 & 6 & 2 & 6 & 7 & 15 & 9 \\
\hline Not Sampling & 15 & 16 & 15 & 14 & 16 & 6 & 2 & 15 & 11 & 1 \\
\hline Not Sampling & 8 & 13 & 8 & 10 & 12 & 11 & 13 & 14 & 2 & 12 \\
\hline Not Sampling & 5 & 2 & 3 & 11 & 4 & 3 & 9 & 10 & 7 & 11 \\
\hline Not Sampling & 7 & 11 & 14 & 15 & 11 & 14 & 14 & 6 & 13 & 2 \\
\hline Not Sampling & 11 & 4 & 6 & 2 & 9 & 7 & 7 & 11 & 9 & 7 \\
\hline Not Sampling & 12 & 8 & 16 & 16 & 3 & 8 & 15 & 9 & 6 & 13 \\
\hline Not Sampling & 14 & 5 & 12 & 6 & 8 & 9 & 10 & 16 & 12 & 5 \\
\hline
\end{tabular}

** Note: Grid nodes for each sampling area in each waste site should be numbered consistently, e.g., begin numbering ${ }_{24}$ the nodes in the northwestern-most node, then number consecutively left to right. 

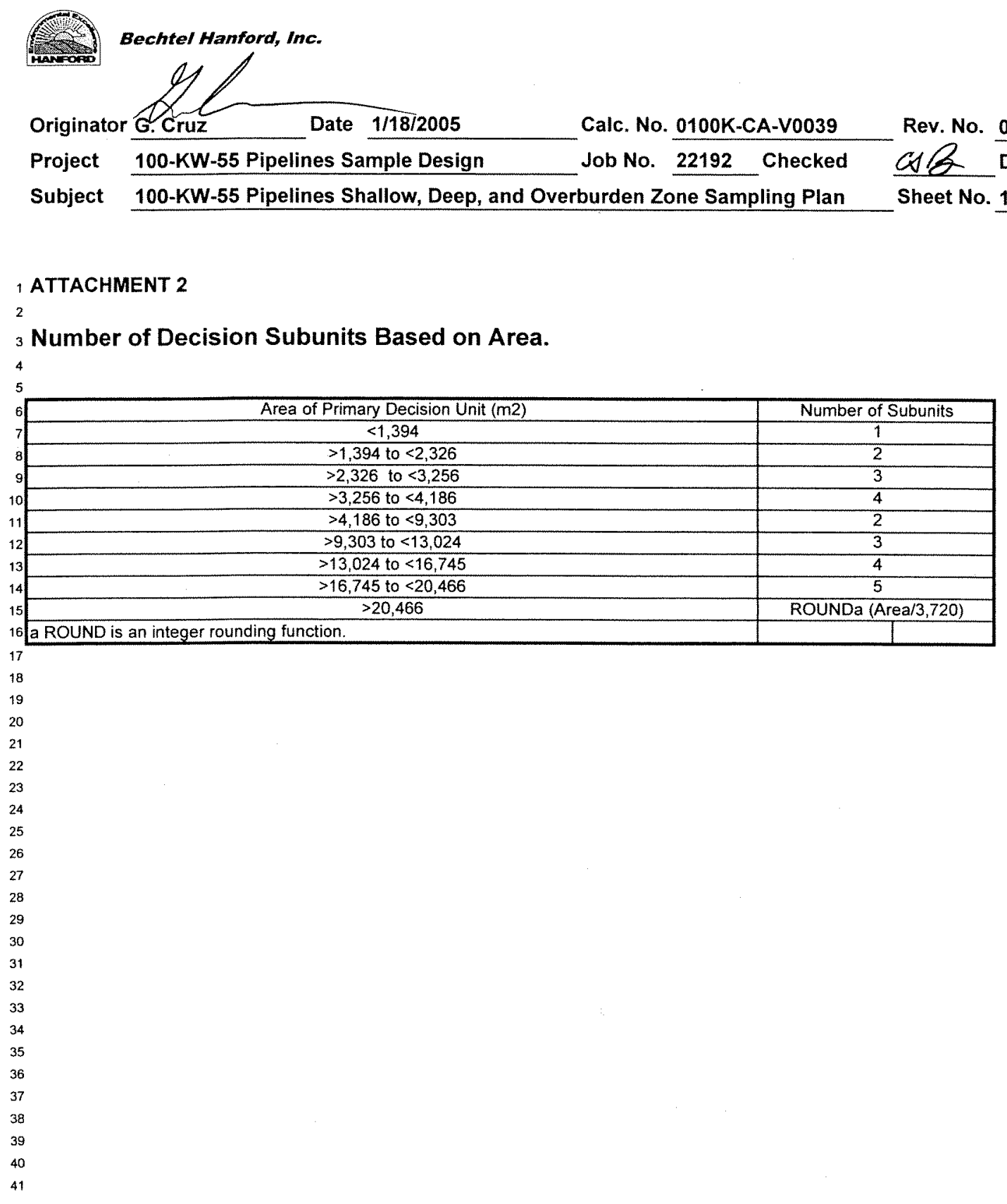
CVP-2005-00006

Rev. 0

C-72 


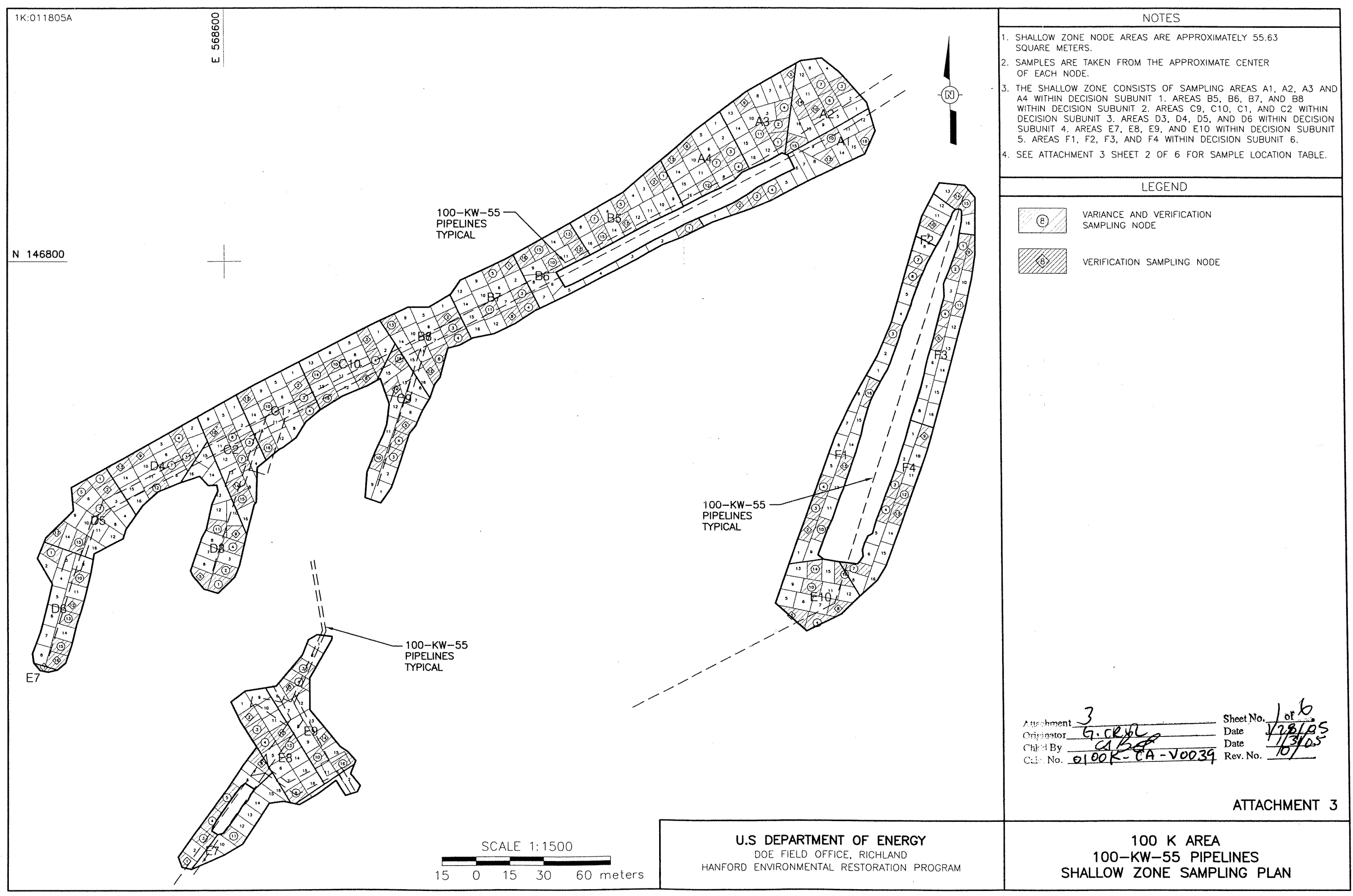




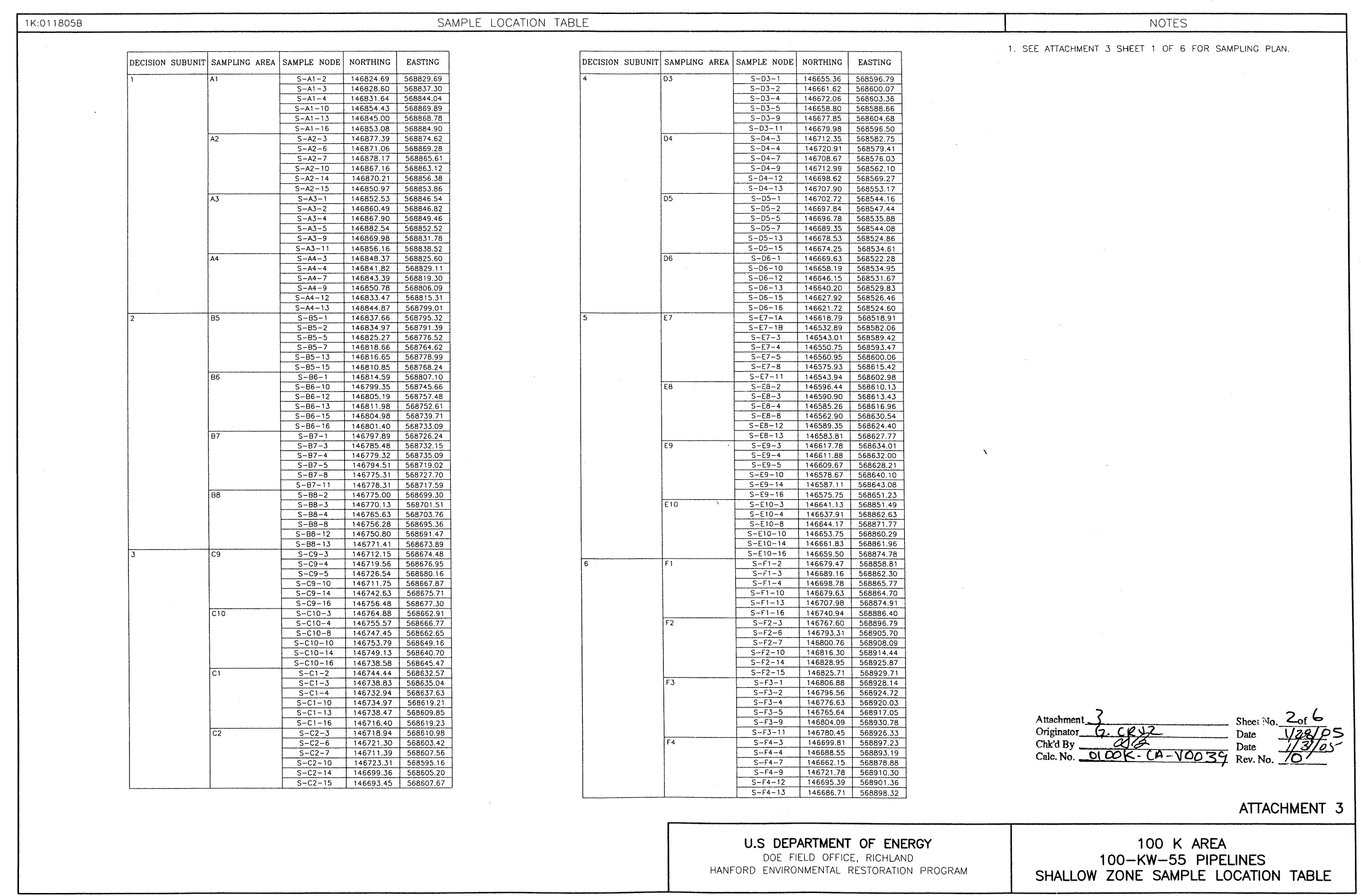




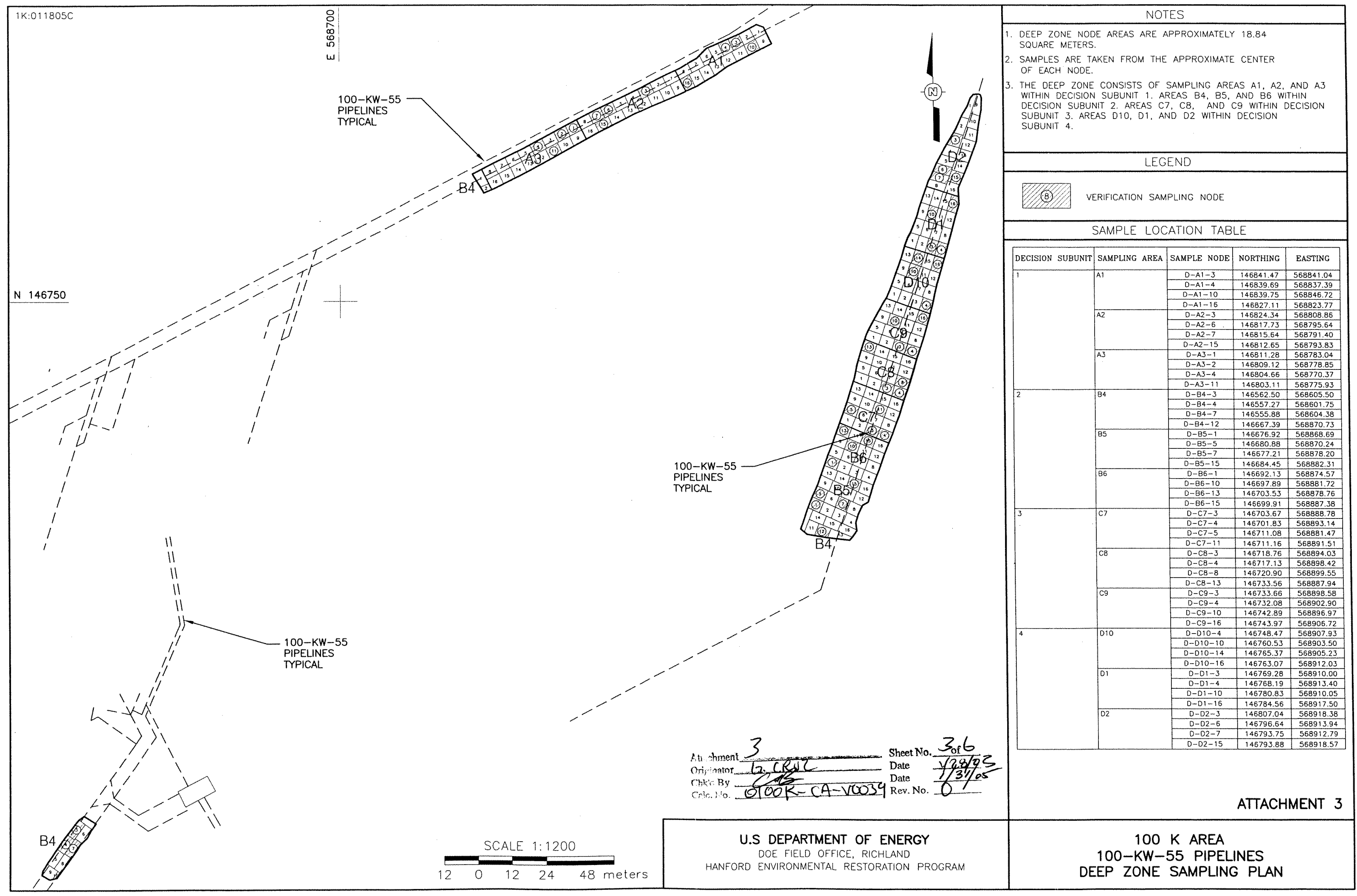




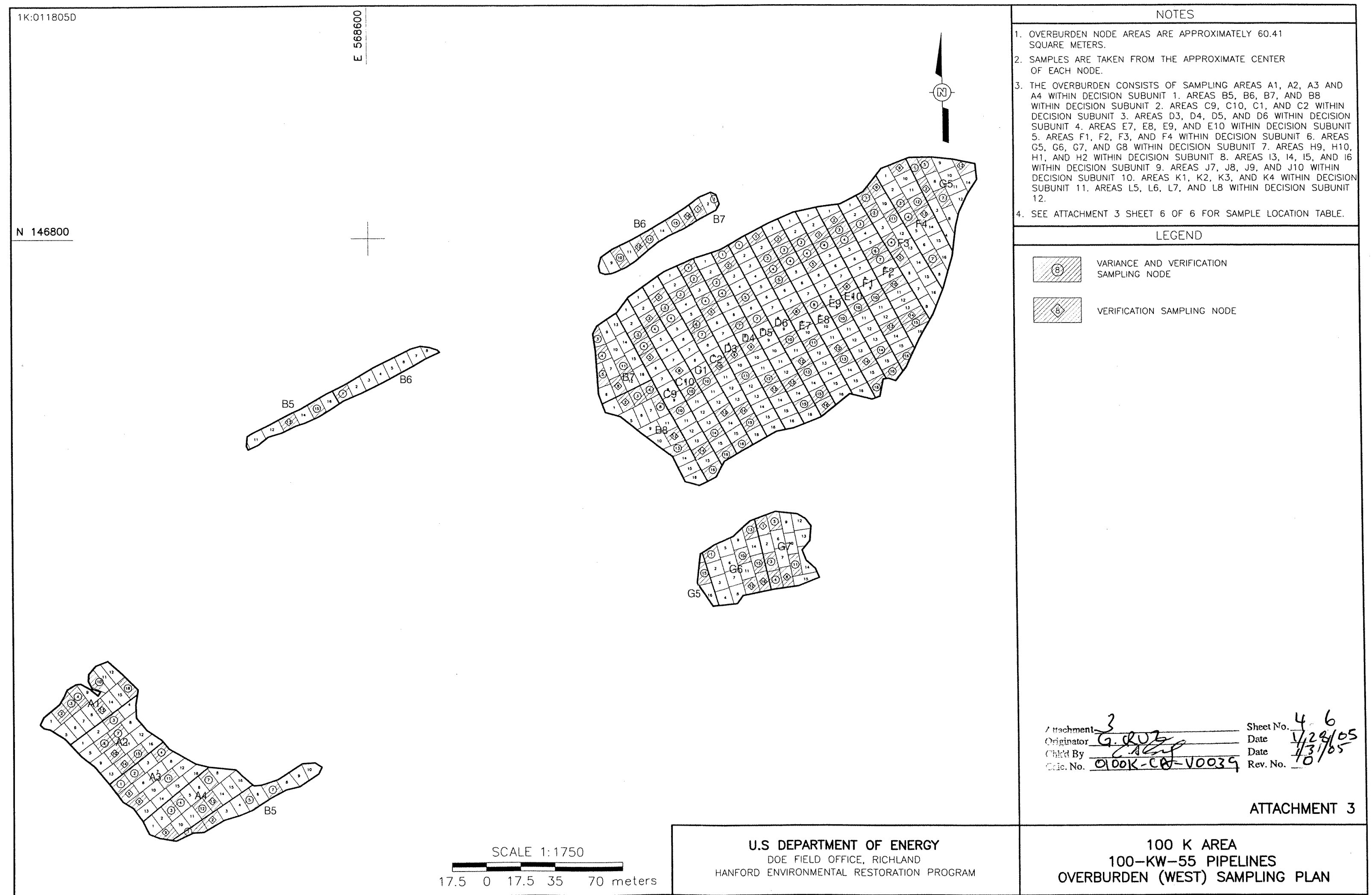




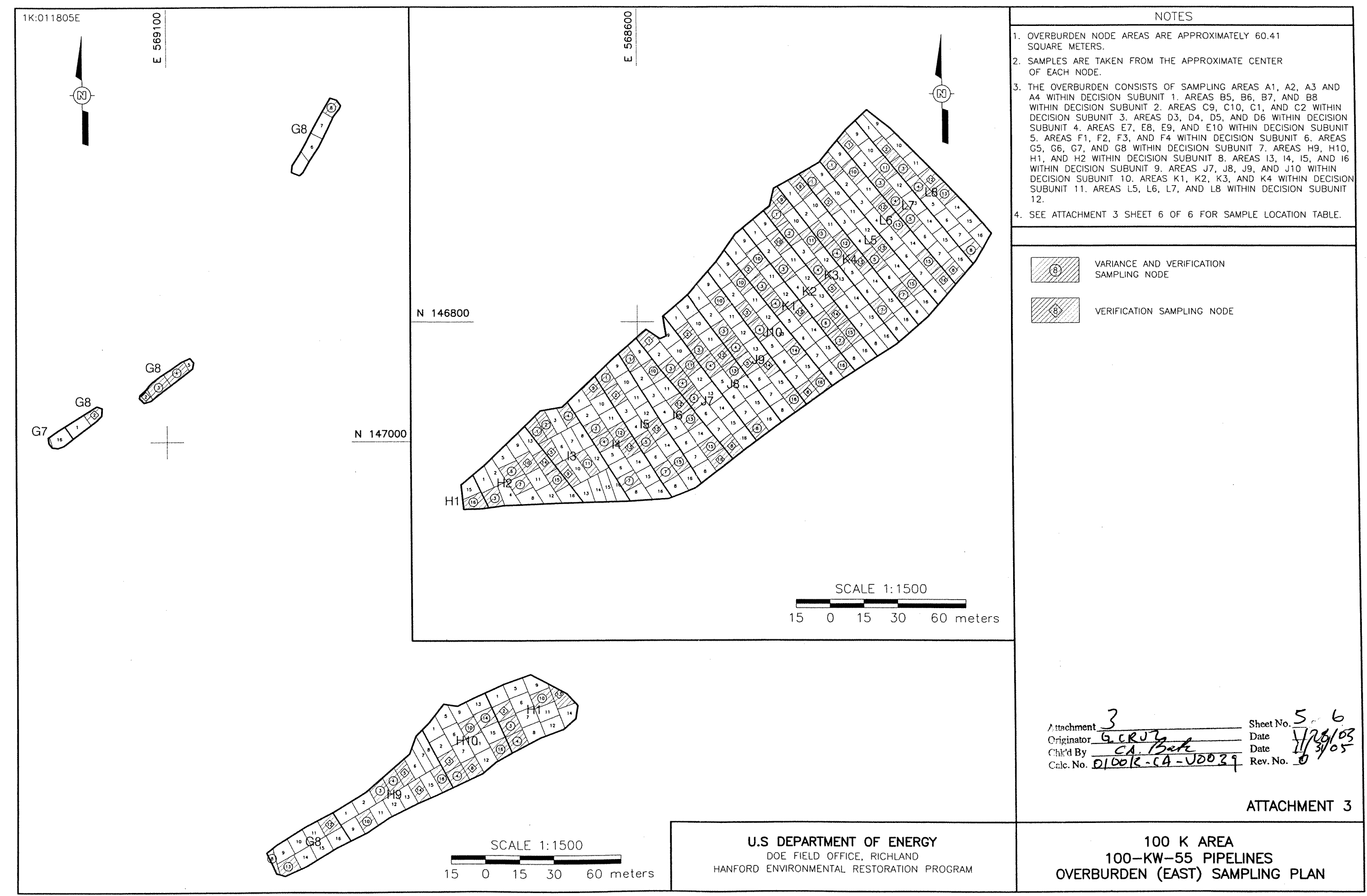




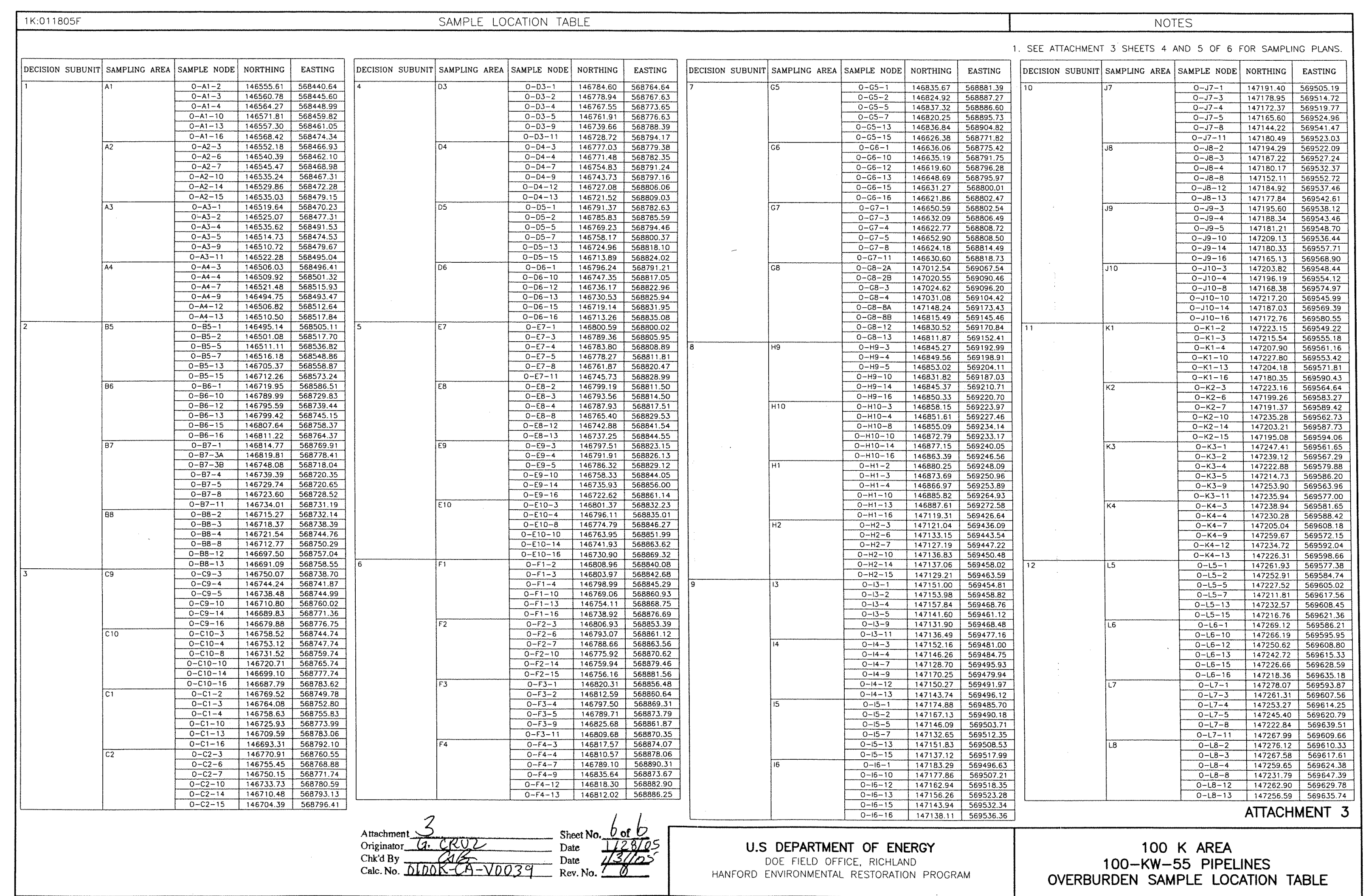


116-KW-3 and 116-KW-4 Discrete Sample Location Map

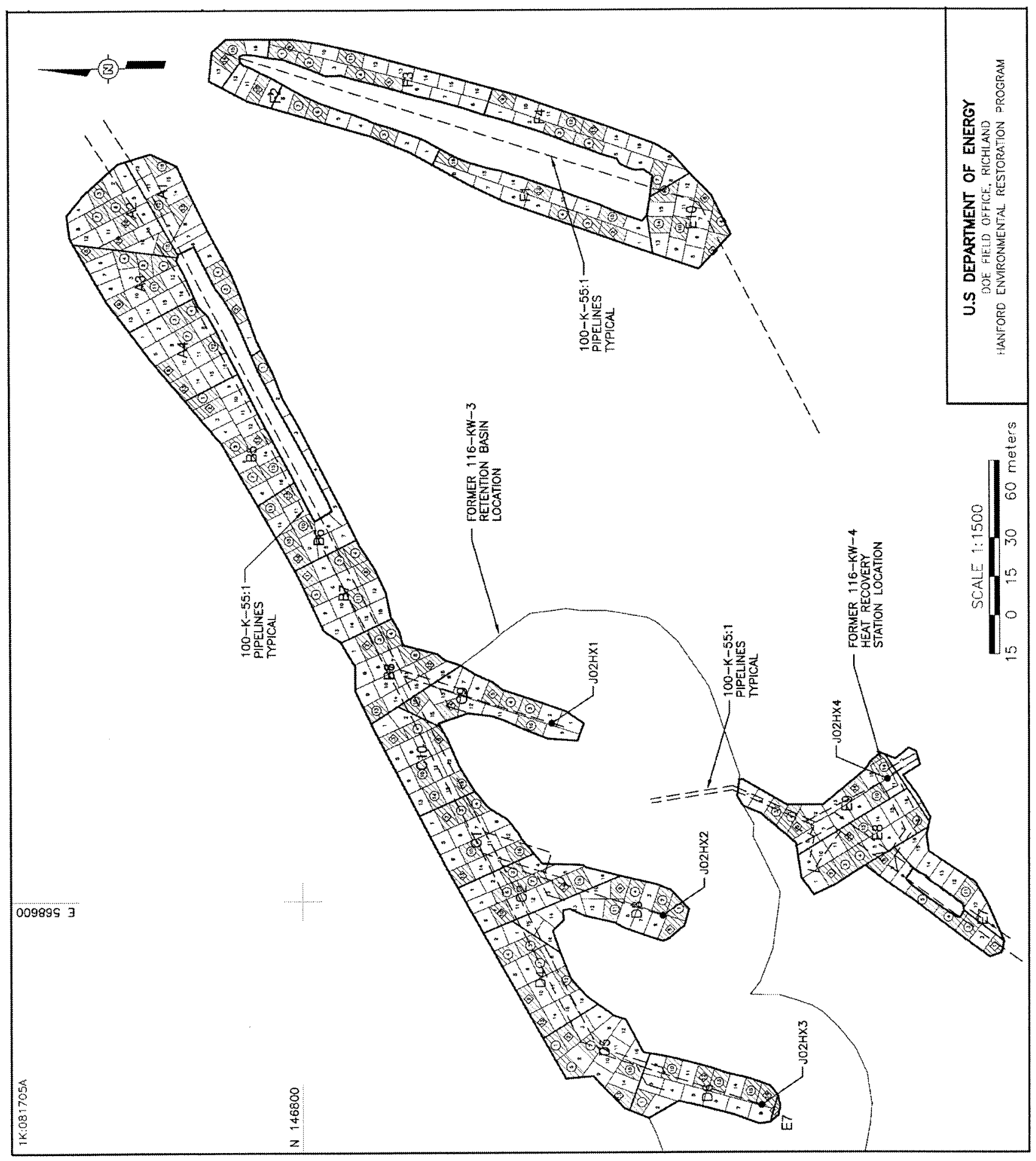


CVP-2005-00006

Rev. 0 


\section{CALCULATION COVER SHEET}

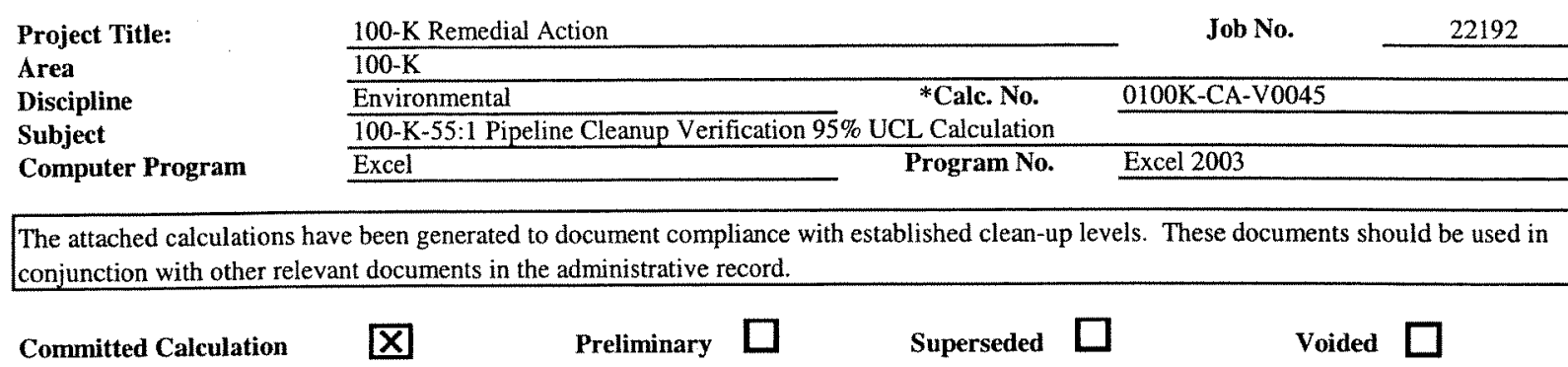

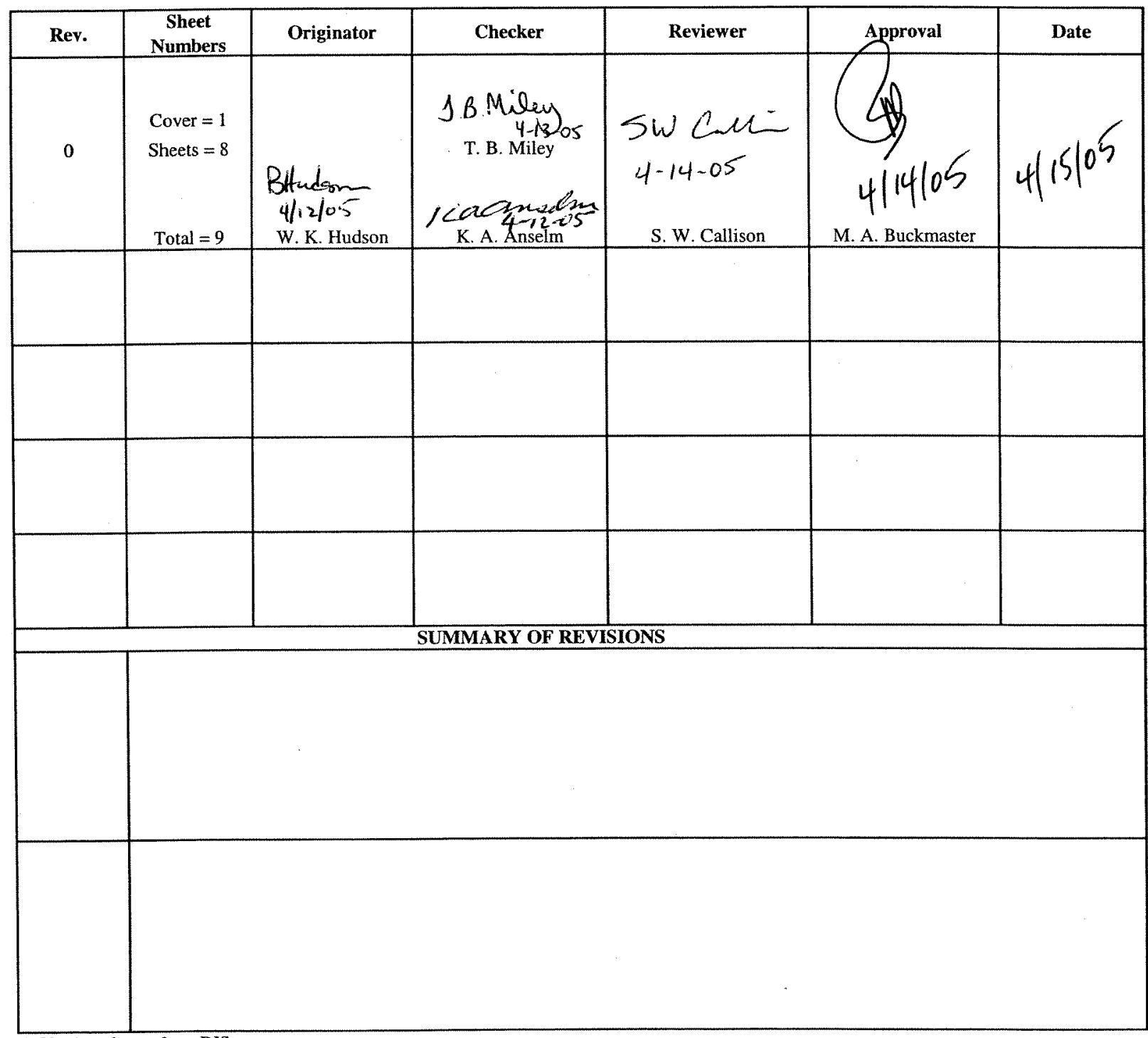

* Obtain calc no. from DIS

DE01437.03 (12/09/2004) 
Originator $\frac{\text { W.K.Hudson }}{100-K \text { Remedial Action }}$ Cerification $95 \%$ UCL Caiculation
Date $04 / 12 / 05$ Job No. 22192
Calc. No. 0100K-CA-V0045

Checked T. B. Miley $1 B M$

checked K.A.Anselm YaCh
Rev. No.

Date $\frac{0}{4-13 \cdot 05}$

Summary

1 Purpose:

Calculate the $95 \%$ upper confidence limit (UCL) to evaluate compliance with cleanup standards for the subject site. Also, calculate the carcinogenic risk for applicable nonradionuclide analyles, 4 (COC)

6 Table of Contents:

7 Sheet 1. Calculation Sheet Summary

Sheet 2 - Calculation Sheet Summary (Continued)

Sheet 3 - Shallow Zone Data

1 Sheet 4 - Deep Zone Data

1 Sheet 5 - Overburden Data

Sheet 6 - Overburden Data (Continued)

Sheet 6 - Split-Duplicate Analysis

Sheet 7 - Split-Duplicate Analysis (Continued)

6 Given/heferences:

1) Sample Results

2) All lookup values, background values, and remedial action goals (RAGs) are taken from the Remedial Design Report/Remedial Action Work Plan (RDRAAWP) (DOE-RL 2002) and

Ecology (1996) unless otherwise specified.

DOE-RL, 2001, 100 Area Remedial Action Sampling and Analysis Plan (SAP), DOE/RL.-96-22, Rev, 3, U.5. Department of Energy, Fichland Operations Office, Richland, Washington

4)

DOE-FL, 2002,
Washington.

Ecology, 1992, Statistical Guidance for Ecology Site Managers, Publication \#92:54, Washington Department of Ecology, Olympia, Washington.

6) Ecology, 1993, Statistical Guidance for Ecology Site Managers, Supplement S-6, Analyzing Site or Background Data with Below-Detection Limit or Below-PQL Values (Censored Data Sets), Publication \#92-54, Washington Department of Ecology, Olympia, Washington.

Sel Cleanup Levels and Risk Calculations (CLARC I), Publication \#94-145, Washington State Department of Ecology, Olympia, Washington.

B) EPA, 1994, USEPA Contract Laboratory Program National Functional Guidelines for Inorganic Data Review, EPA 540/R-94/013, U.S. Environmental Protection Agency. Washington,

D.C.

9) WAC 173-340, 1996, "Model Toxics Control Act--Cleanup," Washington Administrative Code.

Solution:

Calculation methodology is described in Ecology $(1992,1993)$ and below. Use data from attached worksheets to calculate the $95 \%$ UCL for each analyte, the carcinogenic risk, perform the WAC 173-340 3-part test for nonradionuclides, and the RPD for each COC. There are no nonradiunuclide COCs for this site; therefore, the WAC 173-340 3-part test and carcinogenic risk calculations are not required.

Calculation Description:

作

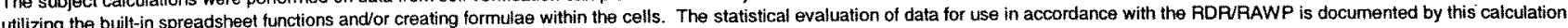
Split and duplicate RPD results are used in evaluation of data quality and are presented in the Cleanup Verfication Package (CVP) for this site.

Methodology:

The maximum value for the (the detection limit value for calculation of the statistics (Ecology 1993). For radionuclide data, calculation of the statistics was done on the reported value. In cases where the laboratory does not report a value below the minimal detectable activity (MDA), half of the MDA is used in the calculation.

The PPD is performed when both the main value and either the duplicate or split values are above detection limits and are greater than 5 times the target detection limit (TDL). The TDL is a These detection limit requirements are located in Table II-2 of the sampling and analysis plan (DOE-AL 2001) where they are re

For quality assurance/quality control (OA/OC) split and duplicate RPD calculations, a value less than $+1-30 \%$ indicates the data compare favorably. For regulatory splits, a threshold of $+1-35 \%$ is performed as part of the evaluation for these two cases involving regulator split data: difference = main - regulator split.

60

the difference is greater than $+/-2$ times the TDL, then further investigation regarding the usability of the data is performed and presented in the data quality assessment section of the applicable 
CVP-2005-00006

Rev. 0

\section{Bechtel Hanford, Inc.}

Originator W. K. Hudson BH Project 100 -K Remedial Action

Subject 100-K-55:1 Pipeline Cleanup Verification 95\% UCL Calculation

Summary (continued)

\section{Results:}

The results presented in the summary tables that follow are for use in RESRAD dose/risk analysis and the CVP for this site.

\begin{tabular}{|l|c|l|l|}
\hline \multicolumn{4}{|c|}{ Result Summary - Shallow Zone } \\
\hline Analyte & Result & Qualifier & Units \\
\hline $\mathrm{Cs}-137$ & $2.7 \mathrm{E}-01$ & & $\mathrm{pCl} / \mathrm{g}$ \\
\hline $\mathrm{Eu}-152$ & $7.3 \mathrm{E}-01$ & & $\mathrm{pCi} / \mathrm{g}$ \\
\hline $\mathrm{Eu}-154$ & $1.2 \mathrm{E}-01$ & & $\mathrm{pCi} / \mathrm{g}$ \\
\hline
\end{tabular}

\begin{tabular}{|c|c|c|c|c|}
\hline \multicolumn{5}{|c|}{ Relative Percent Difference (RPD) Results - } \\
OAOC Analysis Shallow Zone*
\end{tabular}

A blank box indicates the RPD did not need to be calculated.

** Addition discussion on RPDs $>30 \%$ is provided in the data quality assessment section of the CVP.

9

\begin{tabular}{|l|l|l|l|}
\hline \multicolumn{4}{|c|}{ Result Summary - Deep Zone } \\
\hline \multicolumn{1}{|c|}{ Analyte } & Result & Oualifier & Units \\
\hline Cs-137 & $8.2 \mathrm{E}-01$ & & $\mathrm{pCi} / \mathrm{g}$ \\
\hline $\mathrm{Eu}-152$ & $7.4 \mathrm{E}-01$ & & $\mathrm{pCl} / \mathrm{g}$ \\
\hline $\mathrm{Eu}-154$ & $1.2 \mathrm{E}-01$ & & $\mathrm{pCi} / \mathrm{g}$ \\
\hline
\end{tabular}

Eu-154

\begin{tabular}{|c|c|c|c|}
\hline \multicolumn{4}{|c|}{ RPD Results - QAQC Ar } \\
\hline Analyte & $\begin{array}{l}\text { D2 Duplicate } \\
\text { Analysis }\end{array}$ & \multicolumn{2}{|c|}{ D2 Split Analysis } \\
\hline \multicolumn{4}{|l|}{ Cs-137 } \\
\hline \multicolumn{4}{|l|}{ Eu-152 } \\
\hline \multicolumn{4}{|l|}{ Eun 154 } \\
\hline \multicolumn{4}{|c|}{ "A blank box indicates the RPD did not need to be calcule } \\
\hline \multicolumn{4}{|c|}{ Result Summary - Overburden } \\
\hline Analyte & Result & Oualifier & Units \\
\hline Cs-137 & $0(<B G)$ & & $\mathrm{pCil} / 9$ \\
\hline Eu-152 & $3.0 \mathrm{E}-01$ & & $\mathrm{pCi} / \mathrm{g}$ \\
\hline$E u-154$ & $5.3 \mathrm{E}-02$ & & $\mathrm{pCi} / \mathrm{g}$ \\
\hline
\end{tabular}

\begin{tabular}{|c|c|c|c|c|c|c|}
\hline \multicolumn{7}{|c|}{ RPD Results - QAQC Analysis Overburden* } \\
\hline Analyte & $\begin{array}{c}\text { A4 Duplicate } \\
\text { Analysis }\end{array}$ & A4 Split Analysis & F4 Duplicate Analysis & F4 Split Analysis & $\begin{array}{c}\text { K4 Duplicate } \\
\text { Analysis }\end{array}$ & K4 Split Analysis \\
\hline $\mathrm{Cs}-137$ & $11 \%$ & $29 \%$ & & & & \\
\hline Eu-152 & & & & & & \\
\hline$E u-154$ & & & & & & \\
\hline
\end{tabular}

45 * A blank box indicates the RPD did not need to be calculated.

47 Acronyms/abbreviations used on the following pages are listed below.

48 CVP = cleanup verification package

49 HEIS = Hanford Environmental Information System

$50 \mathrm{~J}=$ estimate

$51 \mathrm{MDA}=$ minimal detectable activity

52 MTCA $=$ Model Toxics Control Act

$53 \mathrm{NA}=$ not applicable

$54 \mathrm{POL}=$ practical quantitation limit

$55 \mathrm{Q}=$ qualifier

56 QAQC $=$ quality assurance/quality control

57 RESRAD = RESidual RADioactivity (dose model)

$58 \mathrm{RPD}=$ relative percent difference

$59 \mathrm{TDL}=$ target detection limit

$60 \mathrm{U}=$ undetected

61 WAC $=$ Washington Administrative Code 
CVP-2005-00006

Rev. 0

CALCULATION SHEET

Bechtel Hanford, inc.

Originator W.K. Hudson Pot

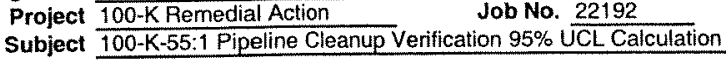

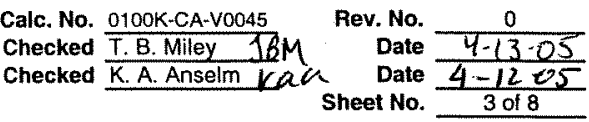

Shallow Zone Sample Data

\begin{tabular}{|c|c|c|c|c|c|c|c|c|c|c|c|}
\hline \multirow{2}{*}{$\begin{array}{c}\text { Sampling } \\
\text { Area }\end{array}$} & \multirow{2}{*}{$\begin{array}{c}\text { HEIS } \\
\text { Number }\end{array}$} & \multirow{2}{*}{$\begin{array}{l}\text { Sample } \\
\text { Date }\end{array}$} & \multicolumn{3}{|c|}{ Cs-137 } & \multicolumn{3}{|c|}{ Eu-152 } & \multicolumn{3}{|c|}{ Eu-154 } \\
\hline & & & $\mathrm{pCi} / \mathrm{g}$ & a & MDA & $\mathrm{pCi} / \mathrm{g}$ & a & MDA & $\mathrm{pCl} / \mathrm{g}$ & $\mathbf{Q}$ & MDA \\
\hline $\mathrm{A} 4$ & J02HK9 & $02 / 23 / 05$ & $3.51 E-01$ & & $5.5 E-02$ & $1.26 \mathrm{E}+00$ & & $1.0 \mathrm{E}-01$ & $2.48 E-01$ & & $1.6 \mathrm{E}-01$ \\
\hline $\begin{array}{c}\text { Duplicate of } \\
\text { J02HKg }\end{array}$ & JO2HLO & $02 / 23 / 05$ & $5.39 \mathrm{E}-01$ & & 5.3E-02 & $2.04 E+00$ & & $1.1 E-01$ & $1.7 \mathrm{E}-01$ & $\mathrm{U}$ & $1.7 E-01$ \\
\hline$F_{4}$ & $\mathrm{JO} 2 \mathrm{HNO}$ & $02 / 28 / 05$ & $3.0 E-02$ & $\mathrm{U}$ & $3.0 \mathrm{E}-02$ & $7.0 \mathrm{E}-02$ & $\mathrm{U}$ & $7.0 \mathrm{E}-02$ & $1.1 \mathrm{E}-01$ & $\mathrm{U}$ & $1.1 \mathrm{E}-01$ \\
\hline $\begin{array}{c}\text { Duplicate of } \\
\text { J02HNO }\end{array}$ & $\mathrm{J02HN}$ & $02 / 28 / 05$ & $3.4 \mathrm{E}-02$ & $U$ & $3.4 \mathrm{E}-02$ & $1.2 \mathrm{E}-01$ & U & $1.2 E-01$ & 1.3E-01 & U & 1.3E-01 \\
\hline A1 & J02HK6 & $02 / 23 / 05$ & $2.9 E-02$ & $\mathrm{U}$ & 3.4E-02 & $8.1 \mathrm{E}-02$ & & $7.3 E-02$ & $9.5 E-02$ & U & $9.5 E-02$ \\
\hline $\mathrm{A} 2$ & $\mathrm{JO} 2 \mathrm{HK} 7$ & $02 / 23 / 05$ & $4.1 \mathrm{E}-02$ & $\bar{U}$ & $4.1 E-02$ & $1,4 E-01$ & & $1.4 \mathrm{E}-01$ & $1.5 \mathrm{E}-01$ & U & $1.5 E-01$ \\
\hline $\mathrm{A3}$ & JO2HK8 & $02 / 23 / 05$ & $1.42 \mathrm{E}-01$ & & $3.6 E-02$ & $4.62 \mathrm{E}-01$ & & $7.8 \mathrm{E}-02$ & $1.1 \mathrm{E}=01$ & U & $1.1 E-01$ \\
\hline 85 & $\mathrm{~J} 02 \mathrm{HL} 1$ & $02 / 23 / 05$ & $6.3 E-02$ & & $3.6 \mathrm{E}-02$ & $1.82 \mathrm{E}-01$ & & $7.4 \mathrm{E}-02$ & $1.3 \mathrm{E}-01$ & $\mathrm{U}$ & 1.3E-01 \\
\hline 86 & J02HL2 & $02 / 23 / 05$ & $3.2 \mathrm{E}-02$ & $\mathrm{U}$ & $3.2 E-02$ & $1.16 \mathrm{E}-01$ & & $7.5 \mathrm{E}-02$ & $1.0 \mathrm{E}-01$ & $\mathrm{U}$ & $1.0 \mathrm{E}=01$ \\
\hline $\mathrm{B} 7$ & J02HL3 & $02 / 23 / 05$ & $4.0 \mathrm{E}-02$ & $\mathrm{U}$ & $4.0 \mathrm{E}-02$ & $1.3 \mathrm{E}-01$ & & $1.3 \mathrm{E}-01$ & $1.6 \mathrm{E}-01$ & $\mathrm{U}$ & $1.6 \mathrm{E}-01$ \\
\hline $\mathrm{B8}$ & $\mathrm{J} 02 \mathrm{HL} 4$ & $02 / 23 / 05$ & $2.26 E-01$ & & $4.7 E-02$ & $6.4 \mathrm{E}-01$ & & $7.8 \mathrm{E}-02$ & $1.3 E-01$ & $\mathrm{U}$ & $1.3 \mathrm{E}=01$ \\
\hline $\mathrm{Cg}$ & $\mathrm{J} 02 \mathrm{HL} 5$ & $02 / 23 / 05$ & $3.4 \mathrm{E}-02$ & $\mathrm{U}$ & $3.4 E-02$ & $6.6 \mathrm{E}-02$ & & $7.5 E-02$ & $1.0 \mathrm{E}-01$ & $\mathrm{U}$ & $1.0 \mathrm{E}-01$ \\
\hline $\mathrm{C} 10$ & J02HL6 & $02 / 23 / 05$ & $2.90 \mathrm{E}-01$ & & $5.5 E-02$ & $3.09 \mathrm{E}-01$ & & $1.0 \mathrm{E}-01$ & $1.6 \mathrm{E}-01$ & U & $1.6 \mathrm{E}=01$ \\
\hline $\mathrm{C} 1$ & J02HL7 & $02 / 23 / 05$ & $3.20 \mathrm{E}-01$ & & $4.3 E-02$ & $7.07 \mathrm{E}-01$ & & $8.5 E-02$ & $1.3 \mathrm{E}-01$ & U & $1.3 \mathrm{E}-01$ \\
\hline $\mathrm{C} 2$ & J02HL8 & $02 / 23 / 05$ & $2.34 \mathrm{E}-01$ & & $4.0 \mathrm{E}-02$ & 2.79E-01 & & $8.4 \mathrm{E}-02$ & 1.1E -01 & $\mathrm{U}$ & $1.1 \mathrm{E}=01$ \\
\hline D3 & $\mathrm{J02HL9}$ & $02 / 24 / 05$ & $4.4 E-02$ & & $3.5 E-02$ & $1.4 \mathrm{E}-01$ & & $9.2 \mathrm{E}-02$ & $1.4 \mathrm{E}-01$ & U & $1.4 \mathrm{E}-01$ \\
\hline D4 & J02HMO & $02 / 24 / 05$ & $3.7 \mathrm{E}-02$ & & $2.8 \mathrm{E}-02$ & $1.61 \mathrm{E}-01$ & & $8.2 E=02$ & $1.3 \mathrm{E}=01$ & $U$ & $1.3 \mathrm{E}=01$ \\
\hline D5 & $\mathrm{JO} 2 \mathrm{HMI}$ & $02 / 24 / 05$ & $6.1 \mathrm{E}-01$ & & $4.5 \mathrm{E}-02$ & $2.28 \mathrm{E}-01$ & & $9.3 E^{-02}$ & $1.6 \mathrm{E}-01$ & $\mathrm{U}$ & $1.6 \mathrm{E}-01$ \\
\hline D6 & $\mathrm{J} 02 \mathrm{HM} 2$ & $02 / 24 / 05$ & $3.98 E-01$ & & $7.7 \mathrm{E}-02$ & $3.87 \mathrm{E}+00$ & & $1.3 E=01$ & $5.21 E-01$ & & $1.7 \mathrm{E}-01$ \\
\hline E7 & $\mathrm{J} 02 \mathrm{HM} 3$ & $02 / 24 / 05$ & $5.7 \mathrm{E}=01$ & & $4,3 \mathrm{E}-02$ & $3.15 \mathrm{E}-01$ & & $7.9 \mathrm{E}-02$ & 1.1E-01 & $\mathrm{U}$ & $1.1 \mathrm{E}-01$ \\
\hline E8 & JO2HM4 & $02 / 24 / 05$ & $2.05 E+01$ & & $3.8 \mathrm{E}-02$ & $1.4 E-01$ & $\mathrm{U}$ & $1.4 \mathrm{E}-01$ & $1.6 \mathrm{E}-01$ & $U$ & $1.6 \mathrm{E}-01$ \\
\hline E9 & J02HM5 & $02 / 24 / 05$ & $8.44 E-01$ & & $6.3 \mathrm{E}-02$ & $1.07 \mathrm{E}+00$ & & $1.2 \mathrm{E}-01$ & $1.9 E-01$ & $\mathrm{U}$ & $1.9 \mathrm{E}-01$ \\
\hline E10 & Jo2HM6 & $02 / 24 / 05$ & 6.3E-02 & $\mathrm{U}$ & $6.3 \mathrm{E}-02$ & $8.2 E-02$ & $u$ & $8.2 E-02$ & $1.2 \mathrm{E}-01$ & $\mathrm{U}$ & $1.2 \mathrm{E}-01$ \\
\hline$F 1$ & J02HM7 & $02 / 24 / 05$ & $3.6 \mathrm{E}-02$ & $\mathrm{U}$ & $3.6 \mathrm{E}-02$ & $1.2 \mathrm{E}-01$ & $\mathrm{U}$ & $1.2 \mathrm{E}-01$ & $1.4 \mathrm{E}-01$ & $\mathrm{U}$ & $1.4 \mathrm{E}-01$ \\
\hline$F_{2}$ & $\mathrm{JO} 2 \mathrm{HMB}$ & $02 / 24 / 05$ & 3.1E-02 & U & $3.1 \mathrm{E}-02$ & $7.5 E-02$ & $\mathrm{U}$ & $7.5 E-02$ & 1.1E-01 & $\mathrm{U}$ & $1.1 \mathrm{E}-01$ \\
\hline$F_{3}$ & J02HM9 & $02 / 24 / 05$ & $3.3 E-02$ & U & $3.3 \mathrm{E}-02$ & $7.8 E-02$ & U & $7.8 \mathrm{E}-02$ & $1.3 \mathrm{E}-01$ & $U$ & $1.3 E-01$ \\
\hline
\end{tabular}

29 Statistical Computation input Data

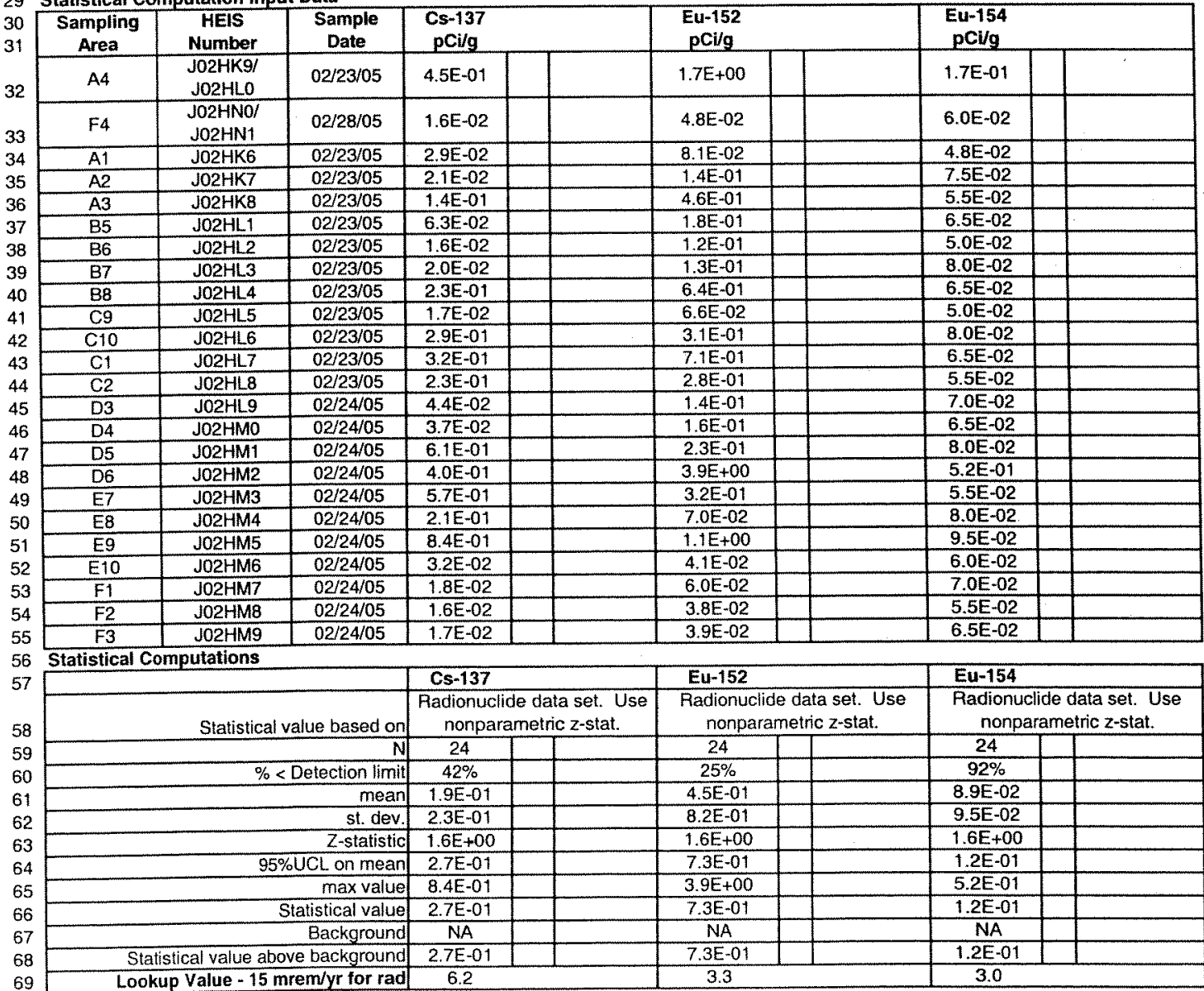

69 Lookup Value - $15 \mathrm{mrem} / \mathrm{yr}$ for rad 
CVP-2005-00006

Rev. 0

Bechtel Hanford, Inc.

Originator W. K. Hudson Project $100-K$ Remedial Action

Subject $100-K-55: 1$ Pipeline Cleanup Verification $95 \%$ UCL Calculation

\section{CALCULATION SHEET}

Date 04/12/05 Job No. 22192
Calc. No. 0100K-CA-V0045 Checked T.B. Miley IBM

Checked K.A. Anselm icaur
Rev. No.

Date 4-13-05

Date $\frac{4-12-05}{4-12-05}$ Sheet No. $\frac{4 \text { of } 8}{4}$

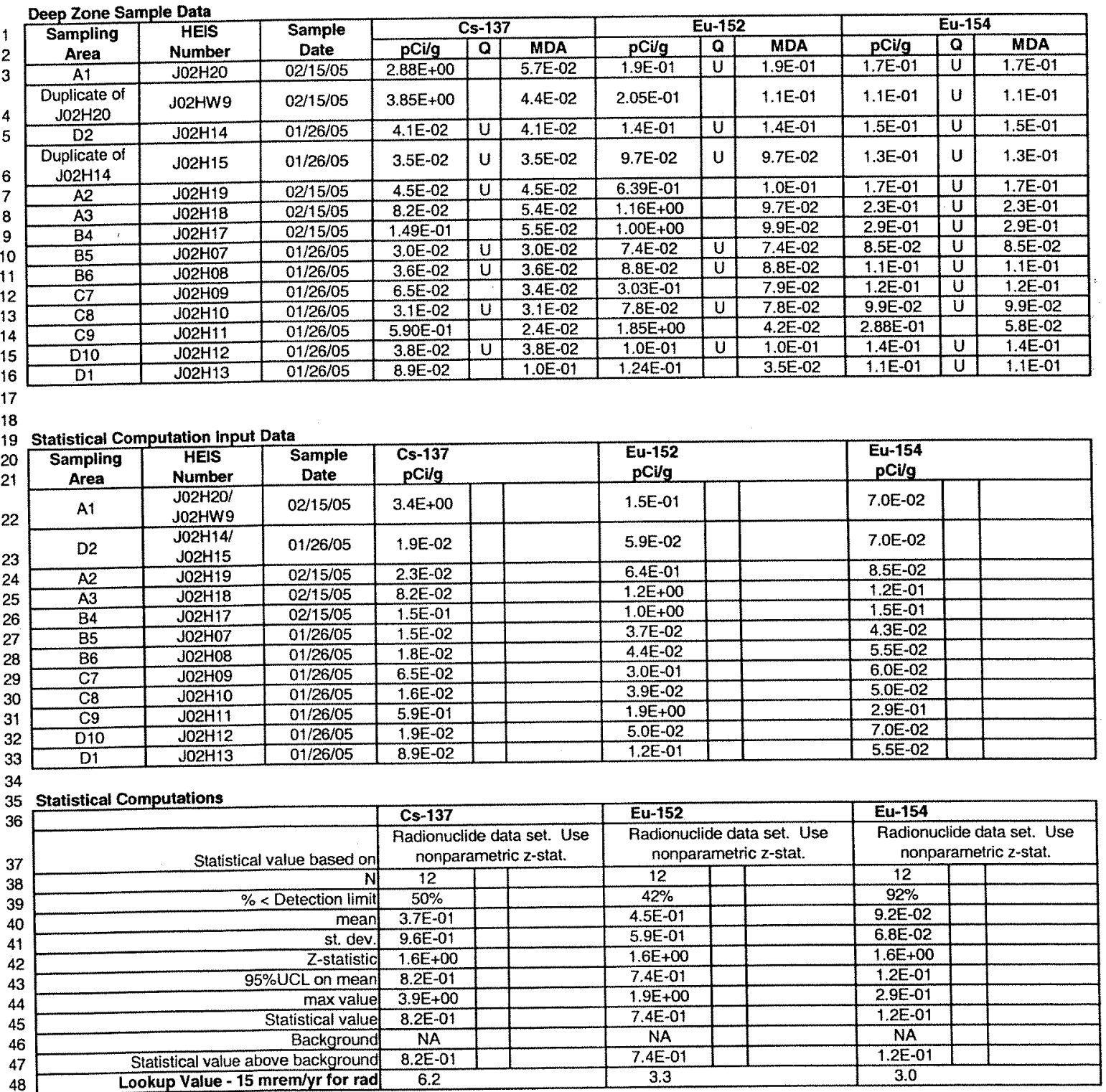




\section{Bechtel Hanford, Inc.}

Originator W. K. Hudson

Subject 100-K-55:1 Pipeline Cleanup Verification $95 \%$ UCL Calculation
CALCULATION SHEET

Date 04/12/05

Calc. No. 0100K-CA-V0045 Checked T. B. Miley $1 B M$ Checked K.A. Anselm Kaa
Rev. No.

Date $4-13.05$

Date $4-12-05$

Sheet No. 5 of 8

\begin{tabular}{|c|c|c|c|c|c|c|c|c|c|c|c|c|}
\hline & \multirow{2}{*}{$\begin{array}{c}\text { Sampling } \\
\text { Area }\end{array}$} & \multirow{2}{*}{$\begin{array}{c}\text { HEIS } \\
\text { Number }\end{array}$} & \multirow{2}{*}{$\begin{array}{c}\text { Sample } \\
\text { Date }\end{array}$} & \multicolumn{3}{|c|}{ Cs-137 } & \multicolumn{3}{|c|}{ Eu-152 } & \multicolumn{3}{|c|}{ Eu-154 } \\
\hline & & & & $\mathrm{pCi} / \mathrm{g}$ & $Q$ & MDA & $\mathrm{pCi} / \mathrm{g}$ & $\mathbf{a}$ & MDA & $\mathrm{pCi} / \mathrm{g}$ & $a$ & MDA \\
\hline & $\mathrm{A} 4$ & J02HN7 & $02 / 18 / 05$ & $1.61 \mathrm{E}+00$ & & $5.4 \mathrm{E}-02$ & $2.65 \mathrm{E}-01$ & & $8.5 E-02$ & $1.2 \mathrm{E}-01$ & $\bar{U}$ & $1.2 \mathrm{E}-01$ \\
\hline & $\begin{array}{c}\text { Duplicate of } \\
\text { J02HN7 }\end{array}$ & J02HNB & $02 / 18 / 05$ & $1.80 E+00$ & & $3.9 \mathrm{E}-02$ & 2.03E-01 & & $8.2 E-02$ & $1.2 \mathrm{E}-01$ & $u$ & 1.2E-01 \\
\hline & $\mathrm{F} 4$ & J02HR8 & $03 / 01 / 05$ & $1.48 \mathrm{E}-01$ & & $4.0 \mathrm{E}-02$ & 1.14E-01 & & $8.3 \mathrm{E}-02$ & $1.3 \mathrm{E}-01$ & $\mathrm{U}$ & $1.3 \mathrm{E}-01$ \\
\hline & $\begin{array}{l}\text { Duplicate of } \\
\text { J02HR8 }\end{array}$ & J02HR9 & $03 / 01 / 05$ & $2.00 \mathrm{E}-01$ & & 4.0E-02 & $1.2 \mathrm{E}-01$ & $u$ & $1.2 \mathrm{E}-01$ & $1.2 \mathrm{E}-01$ & u & $1.2 \mathrm{E}-01$ \\
\hline & $\mathrm{K} 4$ & $\mathrm{~J} 02 \mathrm{HVg}$ & $02 / 17 / 05$ & $3.9 \mathrm{E}-02$ & $U$ & 3.9E-02 & $9.5 \mathrm{E}-02$ & U & $9.5 \mathrm{E}-02$ & $1.4 \mathrm{E}-01$ & $\mathrm{U}$ & $1.4 \mathrm{E}-01$ \\
\hline & $\begin{array}{l}\text { Duplicate of } \\
\text { Jo2HV9 }\end{array}$ & J02HWO & $02 / 17 / 05$ & $4.5 \mathrm{E}-02$ & $u$ & $4.5 E-02$ & $1.4 \mathrm{E}-01$ & u & $1.4 \mathrm{E}-01$ & $1.7 \mathrm{E}-01$ & U & $1.7 \mathrm{E}-01$ \\
\hline & A1 & J02HN4 & $02 / 18 / 05$ & $2.94 \mathrm{E}-01$ & & $4.1 E-02$ & $1.59 \mathrm{E}-01$ & & $7.5 \mathrm{E}-02$ & $1.3 \mathrm{E}-01$ & $\mathrm{U}$ & $1.3 \mathrm{E}-01$ \\
\hline & A2 & J02HN5 & $02 / 18 / 05$ & $1.94 \mathrm{E}-01$ & & $4.4 \mathrm{E}-02$ & $1.44 \mathrm{E}+00$ & & $8.4 \mathrm{E}-02$ & $3.27 \mathrm{E}-01$ & & $1.4 \mathrm{E}-01$ \\
\hline & $\mathrm{A} 3$ & J02HN6 & $02 / 18 / 05$ & $3.95 \mathrm{E}-01$ & & $4.3 \mathrm{E}-02$ & $1.5 \mathrm{E}-01$ & $\bar{U}$ & $1.5 \mathrm{E}-01$ & $1.5 \mathrm{E}-01$ & U & $1.5 \mathrm{E}-01$ \\
\hline & $\mathrm{B} 5$ & J02HN9 & $03 / 03 / 05$ & $3.44 \mathrm{E}-01$ & & $4.4 \mathrm{E}-02$ & 2.03E-01 & & $8.4 \mathrm{E}-02$ & $1.2 \mathrm{E}=01$ & $\mathrm{U}$ & $1.2 E-01$ \\
\hline & $B 6$ & $\mathrm{~J} 02 \mathrm{HPO}$ & $03 / 03 / 05$ & $8.1 \mathrm{E}-02$ & & $3.7 E-02$ & $1.61 \mathrm{E}-01$ & & $8.9 \mathrm{E}-02$ & $1.5 \mathrm{E}-01$ & $U$ & $1.5 E-01$ \\
\hline & B7 & J02HP1 & $03 / 03 / 05$ & $5.60 \mathrm{E}-01$ & & $5.3 \mathrm{E}-02$ & $1.12 \mathrm{E}+00$ & & $8.9 \mathrm{E}-02$ & $1.6 \mathrm{E}-01$ & $\mathrm{U}$ & $1.6 \mathrm{E}-01$ \\
\hline & $B 8$ & $\mathrm{~J} 02 \mathrm{HP} 2$ & $03 / 03 / 05$ & $2.19 \mathrm{E}-01$ & & $3.9 \mathrm{E}-02$ & $1.2 \mathrm{E}-01$ & $\mathrm{U}$ & $1.2 \mathrm{E}-01$ & $1.4 \mathrm{E}-01$ & $U$ & $1.4 \mathrm{E}-01$ \\
\hline & $\mathrm{Cg}$ & J02HP3 & $02 / 22 / 05$ & $4.4 \mathrm{E}-02$ & & $3.0 \mathrm{E}-02$ & $1.2 \mathrm{E}-01$ & $\bar{U}$ & $1.2 \mathrm{E}-01$ & $1.2 \mathrm{E}-01$ & $U$ & $1.2 E-01$ \\
\hline & $\mathrm{C} 10$ & J02HP4 & $02 / 22 / 05$ & $5.3 \mathrm{E}-02$ & & $3.5 \mathrm{E}-02$ & $2.17 E-01$ & & $7.2 \mathrm{E}-02$ & $1.1 \mathrm{E}-01$ & $\bar{U}$ & $1.1 \mathrm{E}-01$ \\
\hline & $\mathrm{C} 1$ & J02HP5 & $02 / 22 / 05$ & $8.2 \mathrm{E}-02$ & & $3.6 \mathrm{E}-02$ & $1.21 \mathrm{E}-01$ & & $7.7 \mathrm{E}-02$ & $1.3 \mathrm{E}-01$ & $\bar{U}$ & $1.3 \mathrm{E}-01$ \\
\hline & $\mathrm{C} 2$ & J02HP6 & $02 / 22 / 05$ & $3.38 \mathrm{E}-01$ & & $4.1 E-02$ & $1.5 \mathrm{E}-01$ & U & $1.5 \mathrm{E}-01$ & $1.4 \mathrm{E}-01$ & $\bar{U}$ & $1.4 \mathrm{E}-01$ \\
\hline & $\mathrm{D} 3$ & J02HP7 & $02 / 22 / 05$ & $2.86 \mathrm{E}-01$ & & $3.0 \mathrm{E}-02$ & $4.82 \mathrm{E}-01$ & & $7.1 \mathrm{E}-02$ & $1.3 \mathrm{E}-01$ & $\mathrm{U}$ & 1.3E-01 \\
\hline & D4 & J02HP8 & $02 / 22 / 05$ & $6.1 \mathrm{E}-02$ & & $3.6 \mathrm{E}-02$ & $1.26 \mathrm{E}-01$ & & $8.1 \mathrm{E}-02$ & $1.2 \mathrm{E}-01$ & $\bar{U}$ & $1.2 \mathrm{E}-01$ \\
\hline & D5 & J02HP9 & $02 / 22 / 05$ & $1.05 \mathrm{E}-01$ & & $3.7 \mathrm{E}-02$ & $1.5 \mathrm{E}-01$ & $\mathrm{U}$ & $1.5 \mathrm{E}-01$ & $1.4 \mathrm{E}-01$ & U & $1.4 \mathrm{E}-01$ \\
\hline & D6 & J02HRO & $02 / 22 / 05$ & $1.52 \mathrm{E}-01$ & & $3.5 \mathrm{E}-02$ & $1.4 \mathrm{E}-01$ & $\mathrm{U}$ & $1.4 \mathrm{E}-01$ & $1.4 \mathrm{E}-01$ & $\bar{U}$ & $1.4 \mathrm{E}-01$ \\
\hline & E7 & J02HA1 & $03 / 01 / 05$ & $1.08 \mathrm{E}-01$ & & $3.6 \mathrm{E}-02$ & $2.65 \mathrm{E}-01$ & & $1.0 \mathrm{E}-01$ & $1.6 \mathrm{E}-01$ & U & $1.6 \mathrm{E}-01$ \\
\hline & E8 & J02HR2 & $03 / 01 / 05$ & $1.62 E+00$ & & $5.0 \mathrm{E}-02$ & $1.24 \mathrm{E}+00$ & & $1.0 \mathrm{E}-01$ & $2.3 \mathrm{E}-01$ & U & $2.3 \mathrm{E}-01$ \\
\hline & E9 & J02HA3 & $03 / 01 / 05$ & $1.36 \mathrm{E}-01$ & & $4.5 \mathrm{E}-02$ & $4.65 \mathrm{E}-01$ & & $1.0 \mathrm{E}-01$ & $1.8 \mathrm{E}-01$ & $\bar{U}$ & $1.8 \mathrm{E}-01$ \\
\hline & E10 & J02HR4 & $03 / 01 / 05$ & $1.69 \mathrm{E}-01$ & & 4.5E-02 & $5.96 E-01$ & & $1.0 \mathrm{E}-01$ & 1.7E-01 & $\mathrm{U}$ & $1.7 \mathrm{E}-01$ \\
\hline & $\mathrm{F} 1$ & J02HR5 & $03 / 01 / 05$ & $1.92 \mathrm{E}-01$ & & $3.8 \mathrm{E}-02$ & $4.08 \mathrm{E}-01$ & & $7.8 \mathrm{E}-02$ & $1.4 \mathrm{E}-01$ & $\mathrm{U}$ & $1.4 \mathrm{E}-01$ \\
\hline & $\mathrm{F} 2$ & J02HRG & $03 / 01 / 05$ & $3.6 \mathrm{E}-02$ & & $3.6 \mathrm{E}-02$ & $1.0 \mathrm{E}-01$ & $\bar{U}$ & $1.0 \mathrm{E}-01$ & $1.3 \mathrm{E}-01$ & $\mathrm{U}$ & $1.3 \mathrm{E}-01$ \\
\hline & $F 3$ & J02HR7 & $03 / 01 / 05$ & $7.6 \mathrm{E}-02$ & & $3.4 \mathrm{E}-02$ & $1.2 \mathrm{E}-01$ & $\mathrm{U}$ & $1.2 \mathrm{E}-01$ & 1.5E-01 & $U$ & $1.5 \mathrm{E}-01$ \\
\hline & G5 & JO2HTO & $03 / 02 / 05$ & $5.3 \mathrm{E}-02$ & $\mathrm{U}$ & $5.3 \mathrm{E}-02$ & $1.3 \mathrm{E}-01$ & $U$ & $1.3 \mathrm{E}-01$ & $1.4 \mathrm{E}-01$ & $\mathrm{U}$ & $1.4 \mathrm{E}-01$ \\
\hline & G6 & $\mathrm{J} 02 \mathrm{HT1}$ & $03 / 02 / 05$ & $3.6 \mathrm{E}-02$ & U & $3.6 \mathrm{E}-02$ & $8.6 \mathrm{E}-02$ & $\mathrm{U}$ & $8.6 \mathrm{E}-02$ & $1.2 \mathrm{E}-01$ & $\bar{U}$ & $1.2 \mathrm{E}-01$ \\
\hline & G7 & J02HT2 & $03 / 02 / 05$ & $5.1 \mathrm{E}-02$ & $\mathrm{U}$ & $5.1 E-02$ & $1.1 \mathrm{E}-01$ & $U$ & $1.1 \mathrm{E}-01$ & $1.6 \mathrm{E}-01$ & $\mathrm{U}$ & $1.6 \mathrm{E}-01$ \\
\hline & G8 & $\mathrm{JO2HT3}$ & $03 / 02 / 05$ & $2.58 \mathrm{E}-01$ & & $3.1 \mathrm{E}-02$ & $5.63 \mathrm{E}-01$ & & $7.2 \mathrm{E}-02$ & $1.1 \mathrm{E}-01$ & $\bar{U}$ & $1.1 \mathrm{E}-01$ \\
\hline & $\mathrm{H9}$ & $\mathrm{J} 02 \mathrm{HT} 4$ & $03 / 02 / 05$ & $2.06 \mathrm{E}-01$ & & $3.1 \mathrm{E}-02$ & $3.33 \mathrm{E}-01$ & & $6.0 \mathrm{E}-02$ & $9.7 \mathrm{E}-02$ & U & $9.7 E-02$ \\
\hline & $\mathrm{H} 10$ & J02HT5 & $03 / 02 / 05$ & $2.56 \mathrm{E}-01$ & & $4.4 \mathrm{E}-02$ & 1.91E-01 & & $8.2 E-02$ & $1.2 \mathrm{E}-01$ & $\bar{U}$ & $1.2 \mathrm{E}-01$ \\
\hline & $\mathrm{H1}$ & J02HT6 & $03 / 02 / 05$ & $1.40 \mathrm{E}-01$ & & $4.1 \mathrm{E}-02$ & $7.60 \mathrm{E}-01$ & & $7.3 \mathrm{E}-02$ & $1.83 \mathrm{E}-01$ & & $1.1 \mathrm{E}-01$ \\
\hline & $\mathrm{H} 2$ & J02HT7 & $03 / 02 / 05$ & $3.58 \mathrm{E}-01$ & & $3.9 \mathrm{E}-02$ & $1.3 \mathrm{E}-01$ & $\mathrm{U}$ & $1.3 \mathrm{E}-01$ & $1.5 \mathrm{E}-01$ & $\bar{U}$ & $1.5 \mathrm{E}-01$ \\
\hline & 13 & J02HT8 & $02 / 18 / 05$ & $1.26 \mathrm{E}-01$ & & $3.0 \mathrm{E}-02$ & $1.1 \mathrm{E}-01$ & $\mathrm{U}$ & $1.1 \mathrm{E}-01$ & $1.4 \mathrm{E}-01$ & $\mathrm{U}$ & $1.4 \mathrm{E}-01$ \\
\hline & 14 & J02HT9 & $02 / 18 / 05$ & $3.34 \mathrm{E}-01$ & & $3.3 \mathrm{E}-02$ & $1.2 \mathrm{E}-01$ & $\mathrm{U}$ & $1.2 \mathrm{E}-01$ & $1.5 \mathrm{E}-01$ & $\mathrm{U}$ & $1.5 \mathrm{E}-01$ \\
\hline & 15 & JO2HVO & $02 / 18 / 05$ & $3.3 \mathrm{E}-02$ & U & $3.3 \mathrm{E}-02$ & $8.1 \mathrm{E}-02$ & $\mathrm{U}$ & $8.1 \mathrm{E}-02$ & $1.2 \mathrm{E}-01$ & $\bar{U}$ & $1.2 \mathrm{E}-01$ \\
\hline & 16 & $\mathrm{JO2HV1}$ & $02 / 18 / 05$ & $8.3 \mathrm{E}-02$ & & $3.0 \mathrm{E}-02$ & $1.1 \mathrm{E}-01$ & $U$ & 1.1E-01 & $1.3 \mathrm{E}-01$ & U & $1.3 \mathrm{E}-01$ \\
\hline & $\sqrt{7}$ & $\mathrm{~J} 02 \mathrm{HV} 2$ & $02 / 17 / 05$ & $3.62 E-01$ & & $3.4 \mathrm{E}-02$ & $7.4 \mathrm{E}-02$ & $U$ & $7.4 \mathrm{E}-02$ & $1.1 \mathrm{E}-01$ & $\mathrm{U}$ & $1.1 \mathrm{E}-01$ \\
\hline & $\sqrt{8}$ & $\mathrm{~J} 02 \mathrm{HV} 3$ & $02 / 17 / 05$ & $5.1 \mathrm{E}-02$ & & $2.0 \mathrm{E}-02$ & $4.3 \mathrm{E}-02$ & & $5.0 \mathrm{E}-02$ & $7.8 \mathrm{E}-02$ & $\bar{U}$ & $7.8 \mathrm{E}-02$ \\
\hline & J9 & $\mathrm{J} 2 \mathrm{HV} 4$ & $02 / 17 / 05$ & $1.51 E-01$ & & $2.6 \mathrm{E}-02$ & $9.4 \mathrm{E}-02$ & $U$ & $9.4 \mathrm{E}-02$ & $1.0 \mathrm{E}-01$ & $\bar{U}$ & $1.0 \mathrm{E}-01$ \\
\hline & $\mathrm{J10}$ & J02HV5 & $02 / 17 / 05$ & $4.59 \mathrm{E}-01$ & & $4.7 \mathrm{E}-02$ & $2.55 \mathrm{E}-01$ & & $9.4 \mathrm{E}-02$ & $1.7 \mathrm{E}-01$ & $\bar{U}$ & $1.7 \mathrm{E}-01$ \\
\hline & $\mathrm{K} 1$ & J02HV6 & $02 / 17 / 05$ & $1.44 \mathrm{E}-01$ & & $3.3 \mathrm{E}-02$ & $8.3 \mathrm{E}-02$ & $\mathrm{U}$ & $8.3 \mathrm{E}-02$ & $1.2 \mathrm{E}-01$ & $\bar{U}$ & $1.2 \mathrm{E}-01$ \\
\hline & $\mathrm{K} 2$ & J02HV7 & $02 / 17 / 05$ & $3.1 E-02$ & & $2.9 \mathrm{E}-02$ & $1.4 \mathrm{E}-01$ & U & $1.4 \mathrm{E}-01$ & 1.5E-01 & $\bar{U}$ & $1.5 \mathrm{E}-01$ \\
\hline & $\mathrm{K} 3$ & $\mathrm{~J} 02 \mathrm{HVB}$ & $02 / 17 / 05$ & $4.2 E-02$ & $\bar{U}$ & $4.2 \mathrm{E}-02$ & $1.0 \mathrm{E}-01$ & $\mathrm{U}$ & $1.0 \mathrm{E}-01$ & $1.6 \mathrm{E}-01$ & 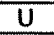 & $1.6 \mathrm{E}-01$ \\
\hline & $L 5$ & J02HW1 & $02 / 17 / 05$ & $3.7 E-02$ & $\mathrm{u}$ & $3.7 \mathrm{E}-02$ & $9.3 \mathrm{E}-02$ & U & $9.3 E-02$ & $1.3 \mathrm{E}-01$ & $U$ & $1.3 \mathrm{E}-01$ \\
\hline & L6 & $\mathrm{J} 02 \mathrm{HW} 2$ & $02 / 17 / 105$ & $3.2 \mathrm{E}-02$ & $\mathrm{U}$ & $3.2 \mathrm{E}-02$ & $7.4 \mathrm{E}-02$ & U & $7.4 \mathrm{E}-02$ & $1.0 \mathrm{E}-01$ & $\bar{U}$ & $1.0 \mathrm{E}-01$ \\
\hline & 17 & J02HW3 & $02 / 17 / 05$ & $3.7 \mathrm{E}-02$ & $\mathrm{U}$ & $3.7 \mathrm{E}-02$ & $1.3 \mathrm{E}-01$ & $U$ & $1.3 \mathrm{E}-01$ & $1.4 \mathrm{E}-01$ & $U$ & $1.4 \mathrm{E}-01$ \\
\hline 3 & L8 & $\mathrm{JO}_{2 \mathrm{HW}}$ & $02 / 17 / 05$ & $3.6 \mathrm{E}-02$ & $U$ & $3.6 \mathrm{E}-02$ & $9.3 \mathrm{E}-02$ & $U$ & $9.3 \mathrm{E}-02$ & $1.3 \mathrm{E}-01$ & U & $1.3 \mathrm{E}-01$ \\
\hline
\end{tabular}


CVP-2005-00006

Rev. 0

\section{Bechtel Hanford, Inc.}

Originator W.K. Hudson BH

Project 100-K Remedial Action
CALCULATION SHEET

Date 04/12/05 Calc. No. 0100K-CA-V0045

Checked K.A.Anselm icaa
Subject 100-K-55:1 Pipeline Cleanup Verification 95\% UCL Calculation

Rev. No.

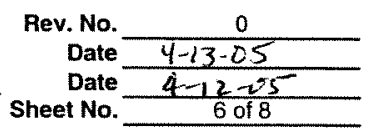

Statistical Computation Input Data (Overburden continued)

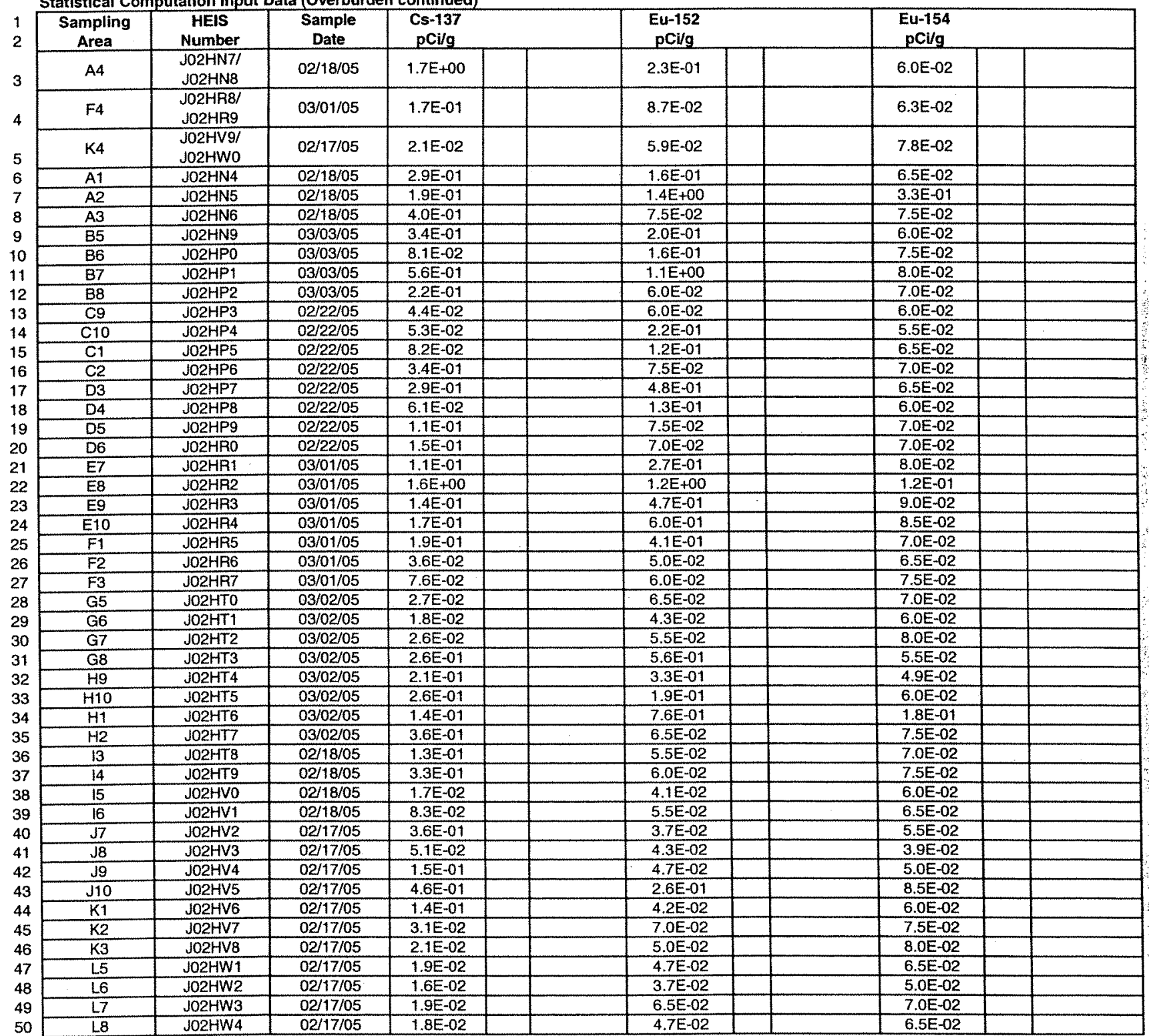

51

52 Statistical Computations

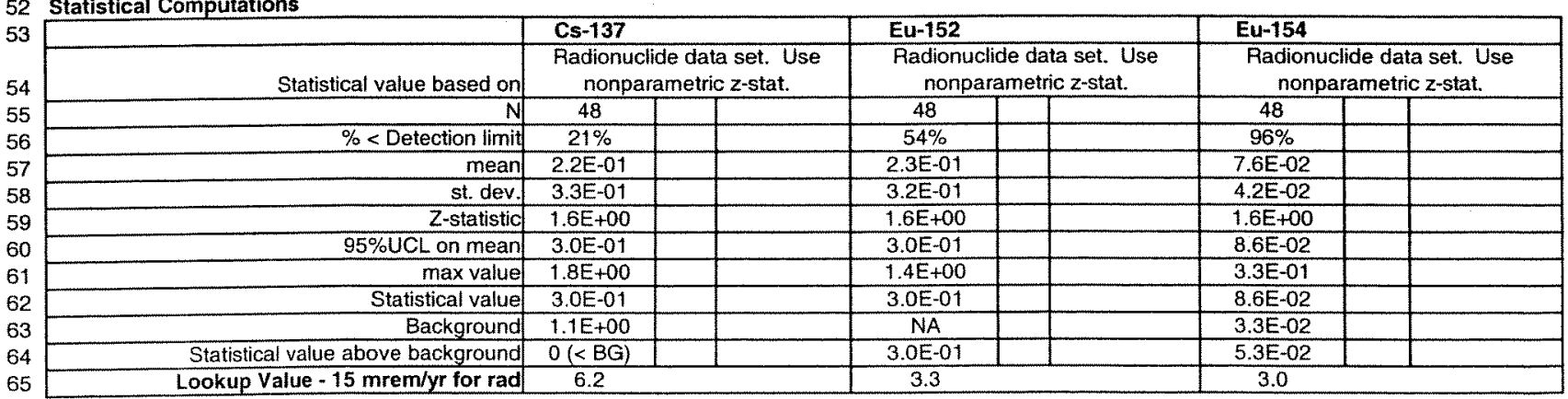


CVP-2005-00006

Rev. 0

\section{Bechtel Hanford, Inc.}

Originator W. K. Hudson Project $100-\mathrm{K}$ Remedial Action

Subject $100-\mathrm{K}-55: 1$ Pipeline Cleanup Verification $95 \%$ UCL Calculation
Date $04 / 12 / 05$

Job No. 22192

\section{CALCULATION SHEET}

Calc. No. 0100K-CA-V0045

Checked T.B. Miley $18 \mathrm{M}$

Checked K.A. Anselm icaa
Rev. No. 0 Date $\frac{0}{4-13-05}$

Date $\frac{4-13-05}{4-2-25}$ Sheet 7 of 8

Split-Duplicate Analysis

1 Shallow Zone Sample Results:

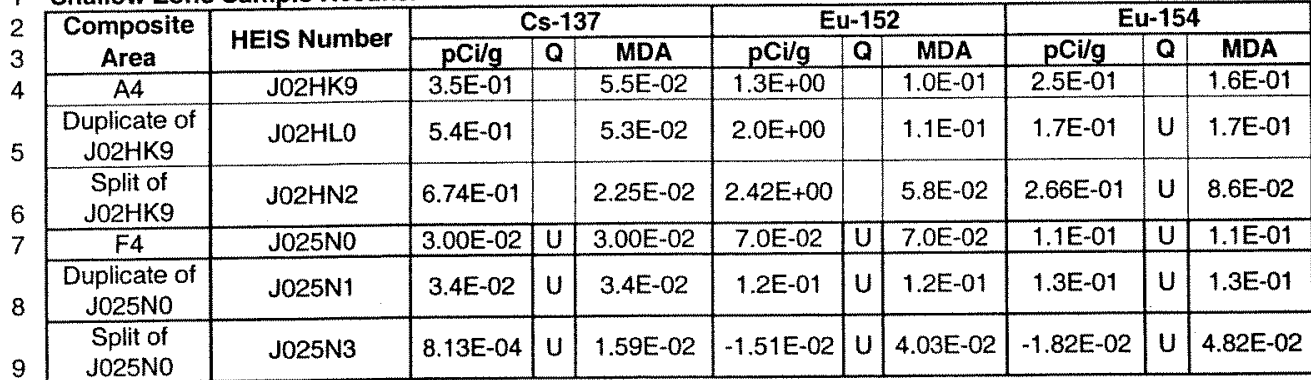

10

11 Shallow Zone Sample Analysis:

\begin{tabular}{|c|c|c|c|c|}
\hline \multicolumn{2}{|c|}{ (TDL) } & 0.1 & 0.1 & 0.1 \\
\hline \multirow{3}{*}{$\begin{array}{l}\text { Duplicate } \\
\text { Analysis } 1\end{array}$} & Both > MDA? & Yes (continue) & Yes (continue) & No-Stop (acceptable) \\
\hline & Both >5xTDL? & No-Stop (acceptable) & Yes (calc RPD) & \\
\hline & $\mathrm{RPD}$ & & $47 \%$ & \\
\hline \multirow{3}{*}{ Split Analysis } & Both > MDA? & Yes (continue) & Yes (continue) & Yes (continue) \\
\hline & Both >5xTDL? & No-Stop (acceptable) & Yes (calc RPD) & No-Stop (acceptable) \\
\hline & $R P D$ & & $63 \%$ & \\
\hline \multirow{3}{*}{$\begin{array}{l}\text { Duplicate } \\
\text { Analysis } 2\end{array}$} & Both > MDA? & No-Stop (acceptable) & No-Stop (acceptable) & No-Stop (acceptable) \\
\hline & Both $>5 \times$ TDL? & & & \\
\hline & $\mathrm{APD}$ & & & \\
\hline \multirow{3}{*}{$\begin{array}{c}\text { Split Analysis } \\
2\end{array}$} & Both > MDA? & No-Stop (acceptable) & No-Stop (acceptable) & No-Stop (acceptable) \\
\hline & Both $>5 \times$ TDL? & & & \\
\hline & $\mathrm{RPD}$ & & & \\
\hline
\end{tabular}

25

26

27 Split-Duplicate Analysis (continued)

28 Deep Zone Sample Results:

\begin{tabular}{|c|c|c|c|c|c|c|c|c|c|c|}
\hline Composite & HFIS Number & & $s-13$ & & & -15 & & & -154 & \\
\hline Area & HEIS Numper & $\mathrm{pCi} / \mathrm{g}$ & $\mathbf{a}$ & MOA & $\mathrm{pCi} / \mathrm{g}$ & $\mathbf{Q}$ & MDA & $\mathrm{pCi} / \mathrm{g}$ & $\mathbf{Q}$ & MDA \\
\hline $\mathrm{D} 2$ & $\mathrm{~J} 02 \mathrm{H}_{14}$ & $4.1 E-02$ & $\mathbf{U}$ & $4.1 \mathrm{E}-02$ & $1.4 \mathrm{E}-01$ & $\mathbf{U}$ & $1.4 E-01$ & $1.5 \mathrm{E}-01$ & $\mathbf{U}$ & $1.5 \mathrm{E}-01$ \\
\hline $\begin{array}{c}\text { Duplicate of } \\
\text { J02 } 114\end{array}$ & J02H15 & $3.5 E-02$ & $\mathbf{U}$ & $3.5 E-02$ & $9.7 E-02$ & $\mathbf{U}$ & 9.7E-02 & $1.3 \mathrm{E}-01$ & U & $1.3 \mathrm{E}=01$ \\
\hline $\begin{array}{c}\text { Split of } \\
102 \mathrm{H14}\end{array}$ & $\mathrm{J} 02 \mathrm{H} 21$ & 2.12E-02 & $\mathbf{U}$ & 2.01E-02 & $-3.34 E-02$ & U & $4.9 E-02$ & $-9.17 E-03$ & U & $6.4 \mathrm{E}-02$ \\
\hline $\mathrm{A} 1$ & $\mathrm{JO} 2 \mathrm{H} 20$ & $2.88 \mathrm{E}+00$ & & $5.7 \mathrm{E}-02$ & $1.9 E-01$ & $\mathrm{U}$ & $1.9 \mathrm{E}-01$ & 1.7E-01 & U & 1.7E-01 \\
\hline $\begin{array}{c}\text { Duplicate of } \\
\text { J02 } 200\end{array}$ & J02HW9 & $3.85 E+00$ & & $4.4 E-02$ & $2.05 E-01$ & & $1.1 \mathrm{E}-01$ & $1.1 \mathrm{E}-01$ & $U$ & 1.1E-01 \\
\hline $\begin{array}{l}\text { Split of } \\
\text { J02H2O }\end{array}$ & $\mathrm{J} 02 \mathrm{H} \times \mathrm{O}$ & $3.86 E+00$ & & $1.61 \mathrm{E}-02$ & 2.13E-01 & U & 5.75E-02 & 1.35E-02 & $\mathbf{u}$ & 4.77E-02 \\
\hline
\end{tabular}

37

38 Deep Zone Sample Analysis:

\begin{tabular}{|c|c|c|c|c|}
\hline \multicolumn{2}{|c|}{ (TDL) } & 0.1 & 0.1 & 0.1 \\
\hline \multirow{3}{*}{$\begin{array}{c}\text { D2 Duplicate } \\
\text { Analysis }\end{array}$} & Both > MDA? & No-Stop (acceptable) & No-Stop (acceptable) & No-Stop (acceptable) \\
\hline & Both $>5 \times T D L ?$ & & & \\
\hline & RPD & & & \\
\hline \multirow{3}{*}{$\begin{array}{c}\text { A1 Duplicate } \\
\text { Analysis }\end{array}$} & Both > MDA? & Yes (continue) & Yes (continue) & Yes (continue) \\
\hline & Both >5xTDL? & Yes (calc RPD) & No-Stop (acceptable) & No-Stop (acceptable) \\
\hline & RPD & $29 \%$ & & \\
\hline \multirow{3}{*}{$\begin{array}{l}\text { D2 Split } \\
\text { Analysis }\end{array}$} & Both > MDA? & No-Stop (acceptable) & No-Stop (acceptable) & No-Stop (acceptable) \\
\hline & Both >5xTDL? & & & \\
\hline & RPD & & & \\
\hline \multirow{3}{*}{$\begin{array}{l}\text { A1 Split } \\
\text { Analysis }\end{array}$} & Both > MDA? & Yes (continue) & No-Stop (acceptable) & No-Stop (acceptable) \\
\hline & Both > 5xTDL? & Yes (calc RPD) & & \\
\hline & RPD & $29 \%$ & & \\
\hline
\end{tabular}


CVP-2005-00006

Rev. 0

CALCULATION SHEET

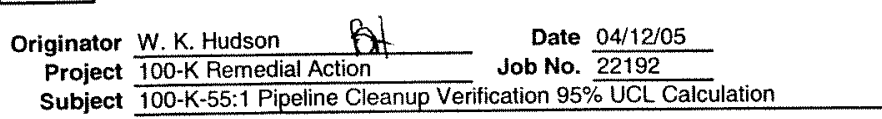

Calc. No. 0100K-CA-V0045 Checked T. B. Miley $15 \mathrm{M}$ Checked K.A.Anselm Kaik

Split-Duplicate Analysis (continued)

1 Overburden Sample Results:

\begin{tabular}{|c|c|c|c|c|c|c|c|c|c|c|}
\hline \multirow{2}{*}{$\begin{array}{c}\text { Composite } \\
\text { Area }\end{array}$} & \multirow{2}{*}{ HEIS Number } & \multicolumn{3}{|c|}{ Cs-137 } & \multicolumn{3}{|c|}{$E \mathrm{u}-152$} & \multicolumn{3}{|c|}{ Eu-154 } \\
\hline & & $\mathrm{pCi} / \mathrm{g}$ & $\mathbf{Q}$ & MDA & $\mathrm{pCi} / \mathrm{g}$ & Q & MDA & $\mathrm{pCi} / \mathrm{g}$ & a & MDA \\
\hline A4 & J02HN7 & $1.61 \mathrm{E}+00$ & & $5.4 E-02$ & $2.65 E-01$ & & $8.5 E-02$ & $1.2 \mathrm{E}-01$ & & $1.2 \mathrm{E}-01$ \\
\hline $\begin{array}{c}\text { Duplicate of } \\
\text { J02HN7 }\end{array}$ & J02HNB & $1.80 E+00$ & & $3.9 E-02$ & 2.03E-01 & & 8.2E-02 & $1.2 E-01$ & & $1.2 E \sim 01$ \\
\hline $\begin{array}{c}\text { Split of } \\
\text { JO2HN7 }\end{array}$ & J02HW5 & $2.15 E+00$ & & 2.26E-02 & $2.90 E-01$ & U & $8.46 E-02$ & $3.26 \mathrm{E}-02$ & U & $7.38 E-02$ \\
\hline$F_{4}$ & J02HAB & $1.48 \mathrm{E}-01$ & & $4.0 \mathrm{E}-02$ & $1.1 \mathrm{E}-01$ & & $8.3 \mathrm{E}-02$ & $1.3 \mathrm{E}-01$ & $\mathrm{U}$ & $1.3 \mathrm{E}-01$ \\
\hline $\begin{array}{c}\text { Duplicate of } \\
\text { JO2HA8 }\end{array}$ & J02HR9 & $2.00 E-01$ & & $4.0 E=02$ & $1.2 E-01$ & $U$ & $1.2 E-01$ & $1.2 E-01$ & $u$ & $1.2 E-01$ \\
\hline $\begin{array}{c}\text { Split of } \\
\text { JO2HR8 }\end{array}$ & J02HW6 & $1.98 E-01$ & & $1.51 E-02$ & 1.57E-01 & $\mathrm{U}$ & $5.12 E-02$ & $2.48 E-03$ & $\mathbf{U}$ & 5.43E-02 \\
\hline$K_{4}$ & J02HV9 & $3.9 E-02$ & $\mathrm{U}$ & $3.9 \mathrm{E}-02$ & $9.5 E-02$ & U & $9.5 E-02$ & $1.4 \mathrm{E}-01$ & $\mathrm{U}$ & $1.4 \mathrm{E}-01$ \\
\hline $\begin{array}{c}\text { Duplicate of } \\
\text { J02HV9 }\end{array}$ & J02HWO & $4.5 E-02$ & $\mathrm{U}$ & $4.5 E-02$ & $1.4 \mathrm{E}-01$ & $U$ & 1.4E-01 & $1.7 \mathrm{E}-01$ & U & $1.7 \mathrm{E}-01$ \\
\hline $\begin{array}{l}\text { Split of } \\
\text { J02HV9 }\end{array}$ & J02HW7 & $1.18 E-02$ & U & $1.52 E-02$ & $2.13 E-02$ & $U$ & $3.9 \mathrm{E}-02$ & $-1.78 E-02$ & $\mathrm{U}$ & $4.15 E-02$ \\
\hline
\end{tabular}

13

14 Overburden Sample Analysis:

\begin{tabular}{|c|c|c|c|c|}
\hline \multicolumn{2}{|c|}{ (TDL) } & 0.1 & 0.1 & 0.1 \\
\hline \multirow{3}{*}{$\begin{array}{c}\text { A4 Duplicate } \\
\text { Analysis }\end{array}$} & Both > MDA? & Yes (continue) & Yes (continue) & No-Stop (acceptable) \\
\hline & Both >5xTDL? & Yes (calc RPD) & No-Stop (acceptable) & \\
\hline & RPD & $11 \%$ & & \\
\hline \multirow{3}{*}{$\begin{array}{c}\text { F4 Duplicate } \\
\text { Analysis }\end{array}$} & Both > MDA? & Yes (continue) & No-Stop (acceptable) & No-Stop (acceptable) \\
\hline & Both >5xTDL? & No-Stop (acceptable) & & \\
\hline & RPD & & & \\
\hline \multirow{3}{*}{$\begin{array}{l}\text { K4 Duplicate } \\
\text { Analysis }\end{array}$} & Both > MDA? & Yes (continue) & No-Stop (acceptable) & No-Stop (acceptable) \\
\hline & Both >5xTDL? & No-Stop (acceptable) & & \\
\hline & RPD & & & \\
\hline \multirow{3}{*}{$\begin{array}{l}\text { A4 Split } \\
\text { Analysis }\end{array}$} & Both > MDA? & Yes (continue) & Yes (continue) & No-Stop (acceptable) \\
\hline & Both $>5 \times T D L ?$ & Yes (calc RPD) & No-Stop (acceptable) & \\
\hline & APD & $29 \%$ & & \\
\hline \multirow{3}{*}{$\begin{array}{l}\text { F4 Split } \\
\text { Analysis }\end{array}$} & Both > MDA? & Yes (continue) & Yes (continue) & No-Stop (acceptable) \\
\hline & Both >5xTDL? & No-Stop (acceptable) & No-Stop (acceptable) & \\
\hline & APD & & & \\
\hline \multirow{3}{*}{$\begin{array}{l}\text { K4 Split } \\
\text { Analysis }\end{array}$} & Both > MDA? & No-Stop (acceptable) & No-Stop (acceptable) & No-Stop (acceptable) \\
\hline & Both > 5xTDL? & & & \\
\hline & APD & & & \\
\hline
\end{tabular}


CVP-2005-00006

Rev. 0

C-90 


\section{CALCULATION COVER SHEET}

Project Title 100-K Remedial Action Job No. 22192

Area 100-K Area

Discipline Environmental

Subject 100-K-55:1 Pipeline RESRAD Calculation

Computer Program RESRAD Program No.
*Calc. No. 0100K-CA-V0046

The attached calculations have been generated to document compliance with established cleanup levels.
These documents should be used in conjunction with other relevant documents in the administrative record.

\section{Committed Calculation $\otimes \quad$ Preliminary $\square \quad$ Superseded $\square \quad$ Voided $\square$}

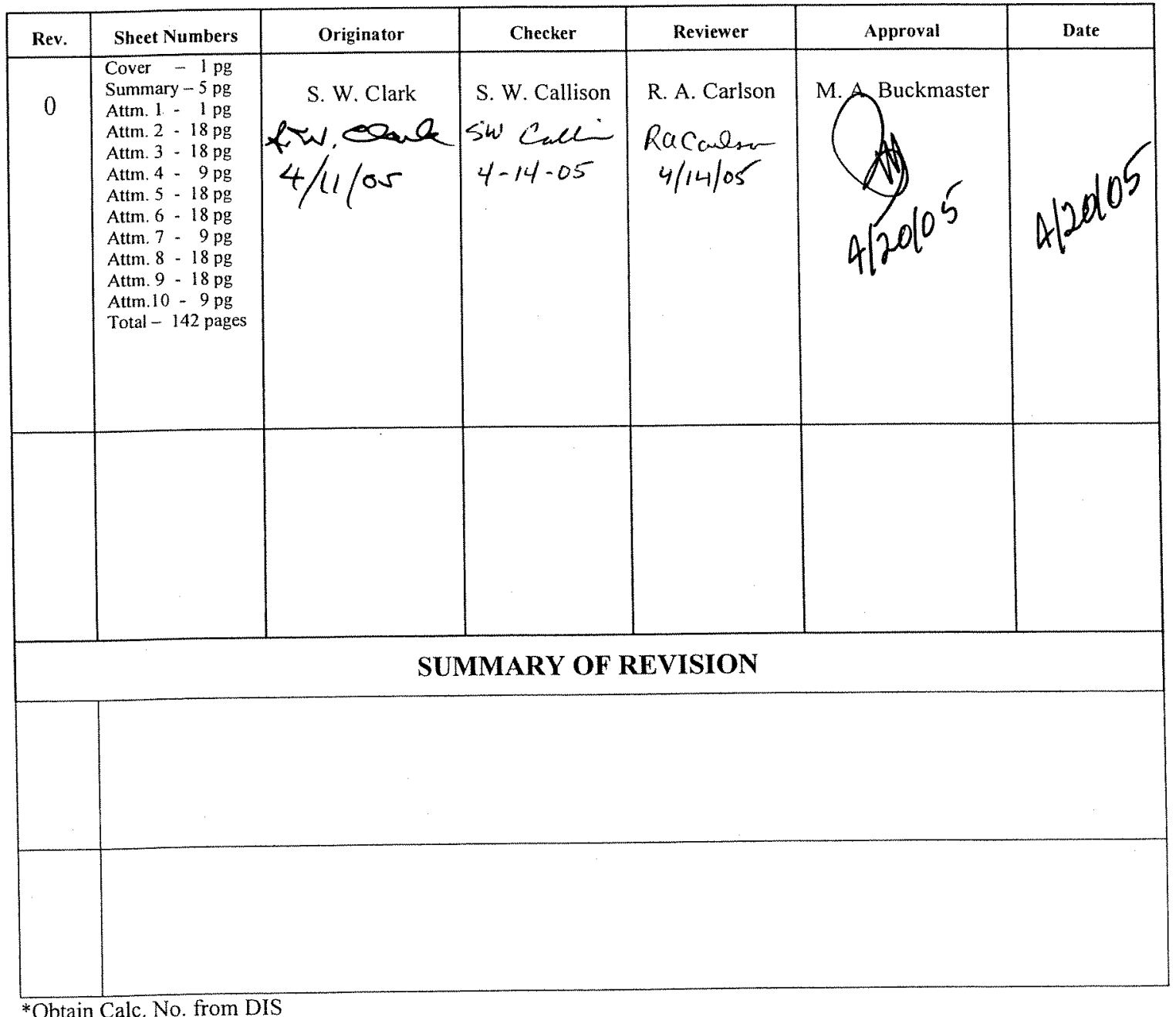

DE01-437.03 (12/09/2004) 
CVP-2005-00006

Rev. 0

Bancorom Bechtel Hanford, Inc. CALCULATION SHEET

\begin{tabular}{|c|c|c|c|c|}
\hline Originator: & S. W. Clark $\quad$ Wue & Date: $4 / 11 / 05$ & Calc. No.: & $0100 \mathrm{~K}-\mathrm{CA}-\mathrm{V} 0046$ \\
\hline & 100-K Remedial Action & Job No: & Checked: & S. W. Callison \\
\hline
\end{tabular}

Subject: $100-\mathrm{K}-55: 1$ Pipeline RESRAD Calculation

\section{PURPOSE:}

Calculate the soil and groundwater concentrations, dose, and risk contributions from remaining radionuclide contaminants in the vadose zone and in stockpiled overburden soil over a period of 1,000 years.

\section{GIVEN/REFERENCES:}

1) Cleanup verification data from 100-K-55:1 Pipeline Cleanup Verification 95\% UCL Calculation, Calculation No. 0100K-CA-V0045, Rev. 0, Bechtel Hanford, Inc., Richland, Washington.

2) Remedial Design Report/Remedial Action Work Plan for the 100 Area (RDR/RAWP), DOE/RL-96-17, Rev. 4, U.S. Department of Energy, Richland Operations Office, Richland, Washington.

3) Radioactive and nonradioactive contaminants of concern from the 100 Area Remedial Action Sampling and Analysis Plan (100 Area SAP), DOE/RL-96-22, Rev. 3, U.S. Department of Energy, Richland Operations Office, Richland, Washington. For the purpose of these RESRAD calculations, the radioactive contaminants of concern (COCs) are cesium-137, europium-152, and europium-154.

4) For the purpose of these RESRAD calculations, there are no nonradionuclide contaminants of concern.

5) RESidual RADioactivity (RESRAD) computer code, version 6.22 , to calculate compliance with residual radioactivity guidelines, developed for the U.S. Department of Energy by the Environmental Assessment Division of Argonne National Laboratory, Argonne, Illinois.

6) Sample design data from the 100-K-55 Pipelines Shallow, Deep, and Overburden Zone Sampling Plan, Calculation No. 0100-CA-V0039, Rev. 0, Bechtel Hanford, Inc., Richland, Washington. For the purpose of these RESRAD calculations, the shallow and deep zone sampling areas identified in the sampling plan calculation brief were summed for a total site area of $24,982 \mathrm{~m}^{2}$. Overburden area is $46,397 \mathrm{~m}^{2}$.

\section{SOLUTION:}

1) Separate RESRAD runs were performed for the shallow and deep vadose zone soil horizons, and for overburden soil. Table 1 shows the waste site parameters used for RESRAD modeling. The model for the RESRAD runs uses the conservative assumption that the residual contamination levels from the data set extend uniformly from the ground surface to groundwater.

2) Table 2 shows the radionuclide activities for each COC. For overburden assays all background concentrations are subtracted from the $95 \%$ UCL values. Input factors for each RESRAD run are shown in the "Summary" section of the RESRAD 
CVP-2005-00006

Rev. 0

Han

CALCULATION SHEET

\begin{tabular}{|c|c|c|c|c|c|c|c|}
\hline Originator: & S.W. Clark,$\alpha<$ & Date: & $4 / 11 / 05$ & Calc. No: & $0100 \mathrm{~K}-\mathrm{CA}-\mathrm{V} 0046$ & Rev.: & 0 \\
\hline Project: & 100-K Remedial Action & Job No: & $2 2 \longdiv { 9 2 }$ & Checked: & S. W. Callison swe & Date: & $4-14-05$ \\
\hline Subject: & \multicolumn{5}{|c|}{ 100-K-55:1 Pipeline RESRAD Calculation } & \multicolumn{2}{|c|}{ Sheet No. 2 of 5} \\
\hline
\end{tabular}

"Mixture Sums and Single Radionuclide Guidelines" printouts in Attachments to this Calculation Summary.

3) The year where the peak dose (or concentration) occurs from each individual radionuclide COC was determined by a preliminary run. This year was then included in all final RESRAD runs. For the direct exposure pathway (i.e. soil ingestion and inhalation and external radiation), the peak year occurred at year zero (year 2005) for all COCs. For the water pathways (i.e., drinking water and food ingestion) the peak year was 42 for cesium-137. The 42 -year time period was included in all of the RESRAD runs.

Table 1. Waste Site Dimensions for RESRAD Modeling

\begin{tabular}{|l|c|c|c|c|c|}
\hline \multicolumn{5}{|c|}{ Table 1. Waste Site Dimensions for RESRAD Modeling } \\
\hline \multicolumn{1}{|c|}{ Parameter } & Units & Shallow Zone & Deep Zone & Overburden & \multicolumn{1}{|c|}{ Comments } \\
\hline Cover Depth & $\mathrm{m}$ & 0 & 4.6 & 0 & \\
\hline Area of Zone & $\mathrm{m}^{2}$ & 24,982 & 24,982 & 46,397 & Based on Sample Design $^{\text {a }}$ \\
\hline $\begin{array}{l}\text { Length Parallel to Aquifer } \\
\text { Flow }\end{array}$ & $\mathrm{m}$ & 204 & 204 & 189 & Based on Sample Design $^{\text {a }}$ \\
\hline Elevation: Zone Surface & $\mathrm{m}$ & 142.0 & 137.4 & 142.0 & NAVD88 $^{-1}$ \\
\hline $\begin{array}{l}\text { Elevation: Groundwater } \\
\text { Thickness: Contaminated } \\
\text { Zone }\end{array}$ & $\mathrm{m}$ & 119.8 & 119.8 & 119.8 & NAVD88 \\
\hline $\begin{array}{l}\text { a Sample design data from the 100-K-55 Pipelines Shallow, Deep, and Overburden Zone Sampling Plan, Calculation } \\
\text { No. 0100K-CA-V0039 }\end{array}$ & 22.2 & 17.6 & 22.2 & $\begin{array}{l}\text { Conservative assumption for } \\
\text { modeling }\end{array}$ \\
\hline
\end{tabular}

\section{METHODOLOGY:}

1) Runs of RESRAD version 6.22 were completed for the shallow zonesusing the radionuclide concentrations shown in Table 2. RESRAD numerical output reports for dose, risk, and concentration for the shallow zones are presented in the Attachments to this calculation summary.

$$
\begin{gathered}
\lambda \text { deepand overbunden } \\
\text { Rac u/iulos' }
\end{gathered}
$$


CVP-2005-00006

Rev. 0

Bechtel Hanford, Inc.

CALCULATION SHEET

\begin{tabular}{|r|l|l|l|l|l|l|l|}
\hline Originator: & S. W. Clark Ru & Date: & $4 / 11 / 05$ & Calc. No.: & $0100 \mathrm{~K}-\mathrm{CA}-$ V0046 & Rev: & 0 \\
\hline Project: & $100-\mathrm{K}$ Remedial Action & Job No: & 22192 & Checked: & S. W. Callison SWL & Date: & $4-14-05$ \\
\hline Subject: & $100-$ K-55:1 Pipeline RESRAD Calculation & & & Sheet No. 3 of 5 \\
\hline
\end{tabular}

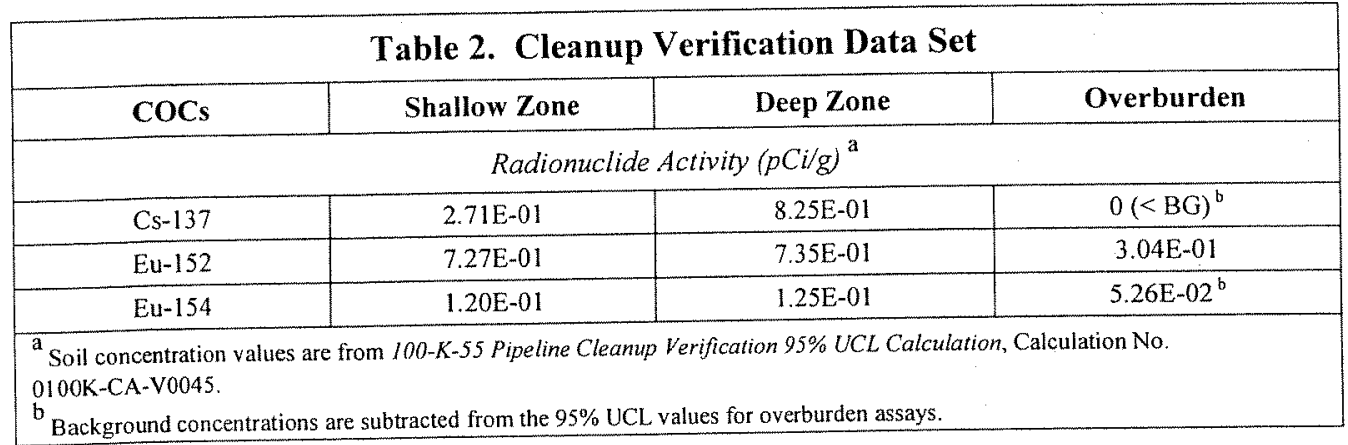

\section{RESULTS:}

\section{1) Radionuclide "All Pathways" Dose Rate}

The "all pathways" (maximum) dose rates are shown in Table 3. The combined shallow and deep zone maximum all-pathways dose is $4.59 \mathrm{mrem} / \mathrm{yr}$ which occurs at year zero (2005). The maximum dose rate from the overburden $(1.66 \mathrm{mrem} / \mathrm{yr})$ also occurs at year zero (2005).

\begin{tabular}{|c|c|c|c|c|c|c|c|c|c|}
\hline \multicolumn{8}{|c|}{ Table 3. All Pathways Dose Rate (mrem/yr) } \\
\hline $\begin{array}{c}\text { RESRAD } \\
\text { Run \# }\end{array}$ & $\begin{array}{c}\text { Vadose Zone } \\
\text { Horizons }\end{array}$ & \multicolumn{2}{c|}{ "All Pathways" Dose Contributions in mrem/yr at Each Time Slice (yr) } \\
\cline { 3 - 10 } & 0 & 1 & 3 & 13 & 42 & 100 & 300 & 1000 \\
\hline 1 & Shallow Zone & $4.59 \mathrm{E}+00$ & $4.37 \mathrm{E}+00$ & $3.95 \mathrm{E}+00$ & $2.41 \mathrm{E}+00$ & $6.60 \mathrm{E}-01$ & $8.88 \mathrm{E}-02$ & $7.06 \mathrm{E}-04$ & $7.09 \mathrm{E}-11$ \\
\hline 2 & Deep Zone & $3.71 \mathrm{E}-25$ & $2.43 \mathrm{E}-04$ & $7.80 \mathrm{E}-04$ & $2.79 \mathrm{E}-03$ & $4.66 \mathrm{E}-03$ & $2.91 \mathrm{E}-03$ & $8.57 \mathrm{E}-05$ & $2.69 \mathrm{E}-11$ \\
\hline $\begin{array}{c}\text { Total All Pathway Dose } \\
\text { Rate (mrem/yr) }\end{array}$ & $4.59 \mathrm{E}+00$ & $4.37 \mathrm{E}+00$ & $3.95 \mathrm{E}+00$ & $2.41 \mathrm{E}+00$ & $6.65 \mathrm{E}-01$ & $9.17 \mathrm{E}-02$ & $7.92 \mathrm{E}-04$ & $9.77 \mathrm{E}-11$ \\
\hline 3 & Overburden & $1.66 \mathrm{E}+00$ & $1.57 \mathrm{E}+00$ & $1.40 \mathrm{E}+00$ & $8.04 \mathrm{E}-01$ & $1.67 \mathrm{E}-01$ & $7.78 \mathrm{E}-03$ & $2.33 \mathrm{E}-07$ & $5.39 \mathrm{E}-16$ \\
\hline
\end{tabular}

\section{2) Radionuclide Excess Cancer Risk}

The radionuclide excess lifetime cancer risk results are shown in Table 4. The maximum excess lifetime cancer risk $\left(5.45 \times 10^{-5}\right)$ for the combined shallow and deep zone occurs at year zero (2005). The maximum excess lifetime cancer risk from the overburden $\left(1.83 \times 10^{-5}\right)$ also occurs at year zero $(2005)$. 
CVP-2005-00006

Rev. 0

\begin{tabular}{|c|c|c|c|c|c|c|c|c|c|}
\hline 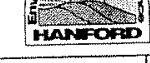 & \multicolumn{6}{|c|}{ CALCULATION SHEET } & & & \\
\hline Originator: & S. W. Clark & \multicolumn{2}{|l|}{ Lwe } & \multicolumn{2}{|c|}{ Date: $4 / 11 / 05$} & Calc. No.: & \multicolumn{2}{|c|}{$0100 \mathrm{~K}-\mathrm{CA}-\mathrm{V} 0046$} & Rev.: \\
\hline Project: & 100-K Remedial & Action & Job & No: $\quad 722$ & 192 & Checked: & \multicolumn{2}{|c|}{ S. W. Callison Swe } & Date: \\
\hline Subject: & \multicolumn{8}{|c|}{ 100-K-55:1 Pipeline RESRAD Calculation } & Sheet $N$ \\
\hline \multicolumn{10}{|c|}{ Table 4. Radionuclide Excess Lifetime Cancer Risk } \\
\hline \multirow{2}{*}{$\begin{array}{l}\text { RESRAD } \\
\text { Run \# }\end{array}$} & \multirow{2}{*}{$\begin{array}{c}\text { Vadose Zone } \\
\text { Horizons }\end{array}$} & \multicolumn{8}{|c|}{ Excess Cancer Risk at Each Time Slice (yr) } \\
\hline & & 0 & 1 & 3 & 13 & 42 & 100 & 300 & 1000 \\
\hline 1 & Shallow Zone & $5.45 \mathrm{E}-05$ & $5.19 \mathrm{E}-05$ & $4.71 \mathrm{E}-05$ & $2.96 \mathrm{E}-05$ & $5 \quad 8.78 \mathrm{E}-06$ & $1.35 \mathrm{E}-06$ & $1.14 \mathrm{E}-08$ & $1.14 \mathrm{E}-15$ \\
\hline 2 & Deep Zone & $5.94 \mathrm{E}-08$ & $6.24 \mathrm{E}-08$ & $6.80 \mathrm{E}-08$ & $8.74 \mathrm{E}-08$ & $8 \mid 9.43 \mathrm{E}-08$ & $5.07 \mathrm{E}-08$ & $1.38 \mathrm{E}-09$ & $4.19 \mathrm{E}-16$ \\
\hline Total Exc & ess Cancer Risk & 5.45E-05 & $5.20 \mathrm{E}-05$ & 4.72E-05 & $2.97 \mathrm{E}-05$ & $58.88 \mathrm{E}-06$ & $1.40 \mathrm{E}-06$ & $1.28 \mathrm{E}-08$ & $1.56 \mathrm{E}-15$ \\
\hline 3 & Overburden & $1.83 \mathrm{E}-05$ & $1.73 \mathrm{E}-05$ & $1.55 \mathrm{E}-05$ & $8.97 \mathrm{E}-06$ & $6 \quad 1.89 \mathrm{E}-06$ & $8.91 \mathrm{E}-08$ & $2.68 \mathrm{E}-12$ & $3.58 \mathrm{E}-21$ \\
\hline
\end{tabular}

\section{3) Radionuclide Groundwater Protection}

The radionuclide concentrations in groundwater calculated by the RESRAD model are summarized in Table 5. The organ specific dose via the groundwater (and river) pathway is presented in a separate calculation brief. Only concentrations are presented here.

Table 5. RESRAD Predicted Radionuclide Groundwater (Well Water) Concentrations

\begin{tabular}{|c|c|c|c|c|c|c|c|c|c|c|}
\hline \multirow{2}{*}{$\begin{array}{l}\text { Radio- } \\
\text { nuclides }\end{array}$} & \multirow{2}{*}{$\begin{array}{c}\text { Vadose Zone } \\
\text { Horizon }\end{array}$} & \multicolumn{8}{|c|}{ Groundwater Concentrations in $\mathrm{pCi} / \mathrm{L}$ at Each Time Slice (yr) } & \multirow{2}{*}{$\begin{array}{l}\text { RAGs } \\
\text { From } \\
\text { RDR }\end{array}$} \\
\hline & & 0 & 1 & 3 & 13 & 42 & 100 & 300 & 1000 & \\
\hline \multirow{4}{*}{ Cs-137 } & Shallow Zone & 0 & $6.82 \mathrm{E}-04$ & $1.95 \mathrm{E}-03$ & $6.72 \mathrm{E}-03$ & $1.11 \mathrm{E}-02$ & $6.91 \mathrm{E}-03$ & $2.03 \mathrm{E}-04$ & $6.34 \mathrm{E}-11$ & \multirow{4}{*}{60} \\
\hline & Deep Zone & 0 & $2.08 \mathrm{E}-03$ & $5.94 \mathrm{E}-03$ & $2.04 \mathrm{E}-02$ & $3.38 \mathrm{E}-02$ & $2.11 \mathrm{E}-02$ & $6.20 \mathrm{E}-04$ & $1.94 \mathrm{E}-10$ & \\
\hline & Total & 0 & $2.76 \mathrm{E}-03$ & $7.90 \mathrm{E}-03$ & $2.72 \mathrm{E}-02$ & $4.49 \mathrm{E}-02$ & $2.80 \mathrm{E}-02$ & $8.24 \mathrm{E}-04$ & $2.58 \mathrm{E}-10$ & \\
\hline & Overburden & 0 & 0 & 0 & 0 & 0 & 0 & 0 & 0 & \\
\hline \multirow{2}{*}{ Eu-152 } & Total & 0 & 0 & 0 & 0 & 0 & 0 & 0 & 0 & \multirow{2}{*}{200} \\
\hline & Overburden & 0 & 0 & 0 & 0 & 0 & 0 & 0 & 0 & \\
\hline \multirow{2}{*}{ Eu-154 } & Total & 0 & 0 & 0 & 0 & 0 & 0 & 0 & 0 & \multirow{2}{*}{60} \\
\hline & Overburden & 0 & 0 & 0 & 0 & 0 & 0 & 0 & 0 & \\
\hline
\end{tabular}

\section{CONCLUSIONS:}

- The maximum combined all-pathways dose rate for the shallow zone and deep zone is $4.59 \mathrm{mrem} / \mathrm{yr}$ and occurs at year zero (2005).

- The dominant pathway for the dose rate is direct external exposure.

- The primary radionuclide contributing to the direct exposure pathway is europium-152.

- None of the site COCs are projected to exceed remedial action goals (RAGs).

- Maximum lifetime cancer risk $\left(5.45 \times 10^{-5}\right)$ for the combined shallow zone and deep zone occurs at year zero (2005). 


\begin{tabular}{|c|c|c|c|c|c|c|}
\hline Originator: & S. W. Clark $\Omega N$ & Date: $\quad 4 / 1 / 65$ & Calc. No: & $0100 \mathrm{~K}-\mathrm{CA}-\mathrm{V} 0046$ & Rev: & 0 \\
\hline Project: & 100-K Remedial Action & Job No: 722192 & Checked: & S. W. Callison Swe & Date: & $4-14-05$ \\
\hline Subject: & \multicolumn{4}{|c|}{ 100-K-55:1 Pipeline RESRAD Calculation } & \multicolumn{2}{|c|}{ Sheet No. 5 of 5} \\
\hline
\end{tabular}

- Among the radionuclide contaminants of concern only cesium-137 is calculated to reach groundwater in the 1,000 years of the RESRAD model run. Cesium-137 is predicted to reach groundwater at concentrations significantly below the RAGs.

\section{ATTACHMENTS:}

1. Graphic showing 100-K-55:1 Cleanup Verification Model (1 page)

2. RESRAD Output: 100-K-55:1 Shallow Zone Radionuclides, Mixture Sums and Single Radionuclide Guidelines (18 pages)

3. RESRAD Output: 100-K-55:1 Shallow Zone Radionuclides, Intake Quantities and Health Risk Factors (18 pages)

4. RESRAD Output: 100-K-55:1 Shallow Zone Radionuclides, Concentration of Radionuclides, ( 9 pages)

5. RESRAD Output: 100-K-55:1 Deep Zone Radionuclides, Mixture Sums and Single Radionuclide Guidelines (18 pages)

6. RESRAD Output: 100-K-55:1 Deep Zone Radionuclides, Intake Quantities and Health Risk Factors (18 pages)

7. RESRAD Output: 100-K-55:1 Deep Zone Radionuclides, Concentration of Radionuclides, (9 pages)

8. RESRAD Output: 100-K-55:1 Overburden Radionuclides, Mixture Sums and Single Radionuclide Guidelines (18 pages)

9. RESRAD Output: 100-K-55:1 Overburden Radionuclides, Intake Quantities and Health Risk Factors (18 pages)

10. RESRAD Output: 100-K-55:1 Overburden Radionuclides, Concentration of Radionuclides, ( 9 pages) 


\section{0-K-55 Pipelines Cleanup Verification Model}

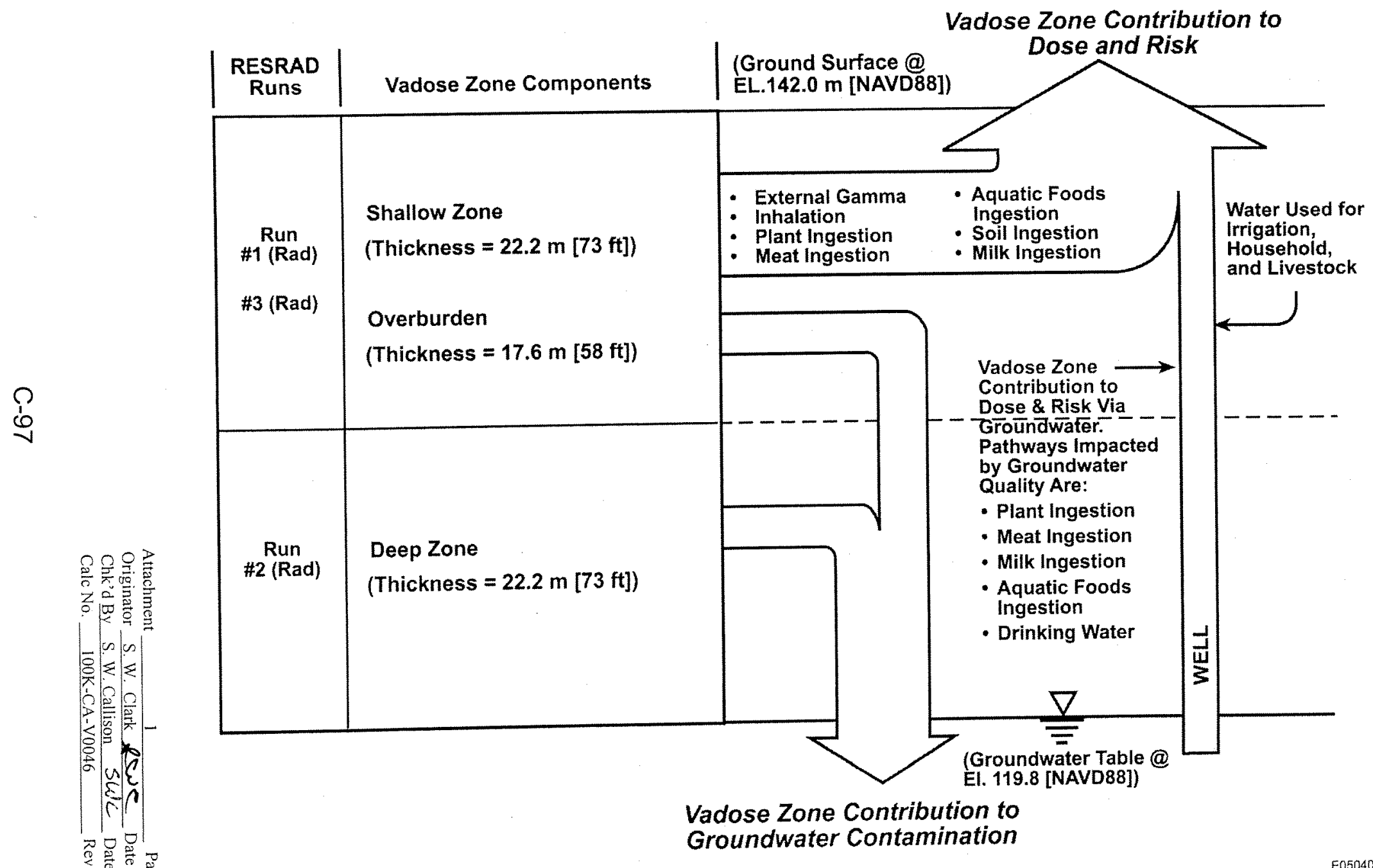


CVP-2005-00006

Rev. 0

C-98 
CVP-2005-00006

Rev. 0

CALCULATION COVER SHEET

(Sample Design, Verification, or Waste Characterization Calculation)

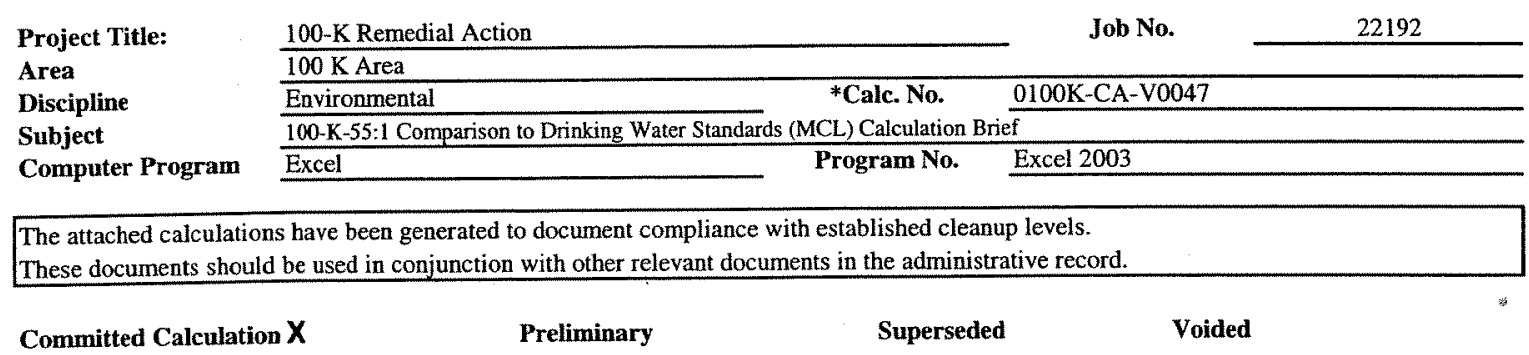

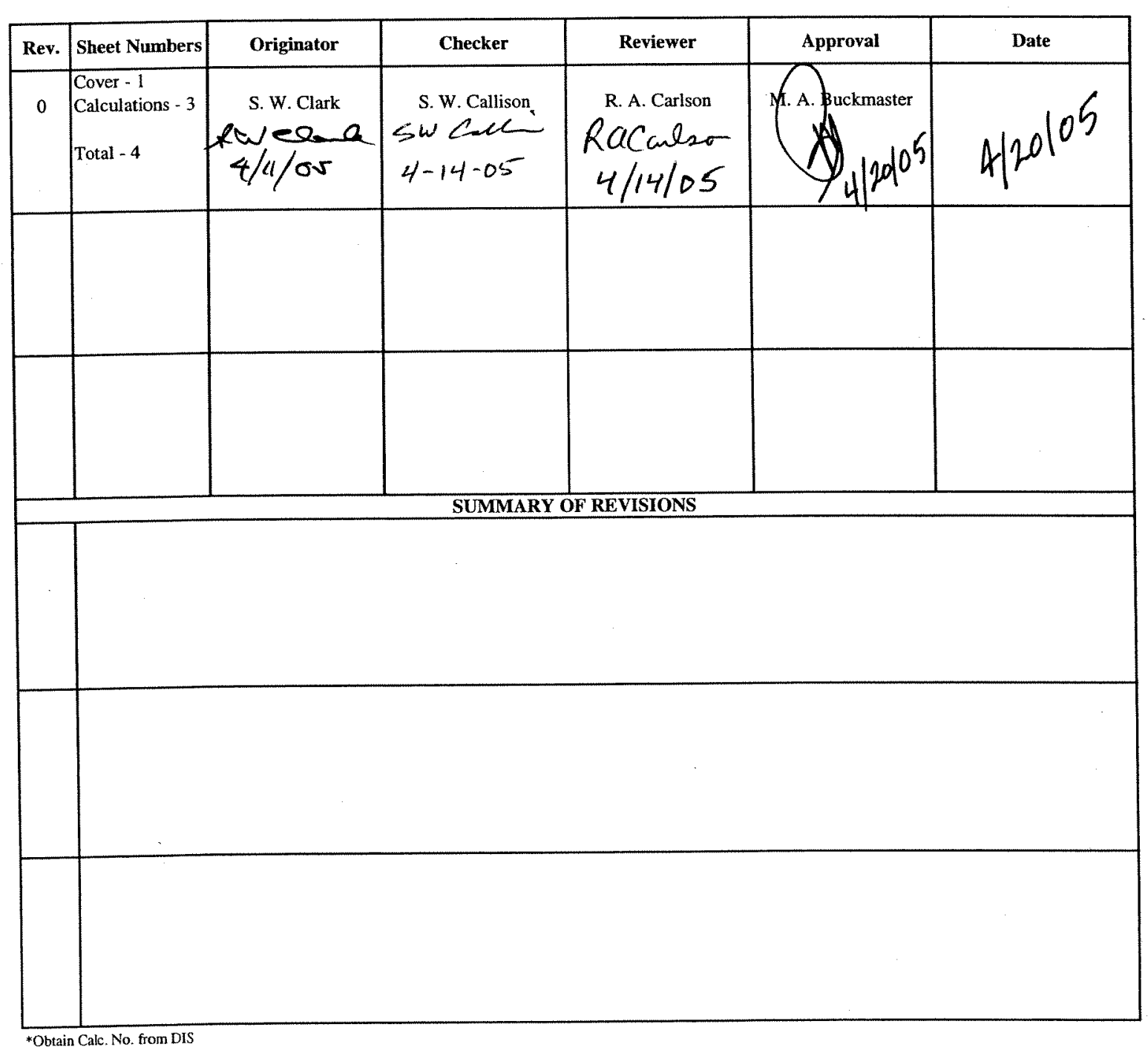


CVP-2005-00006

Rev. 0

Bechtel Hanford, inc.

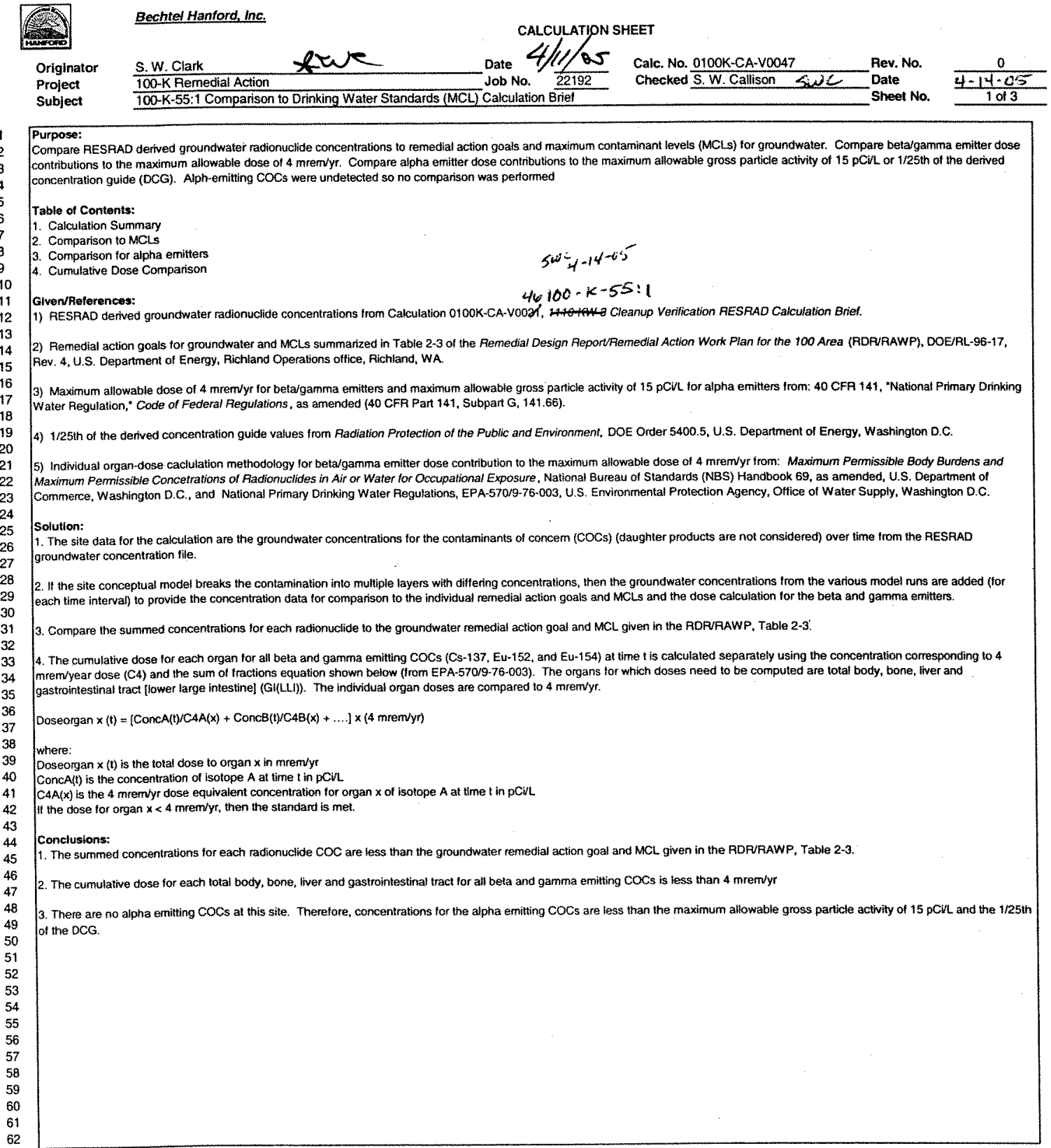




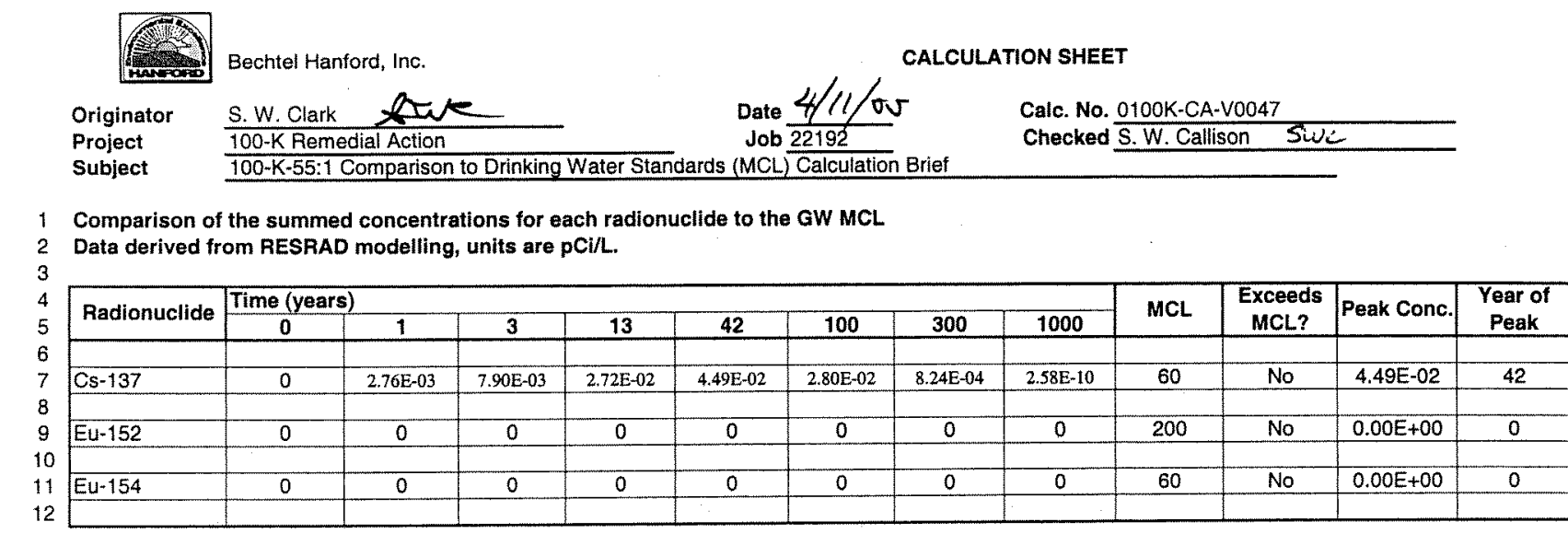


CVP-2005-00006

Rev. 0

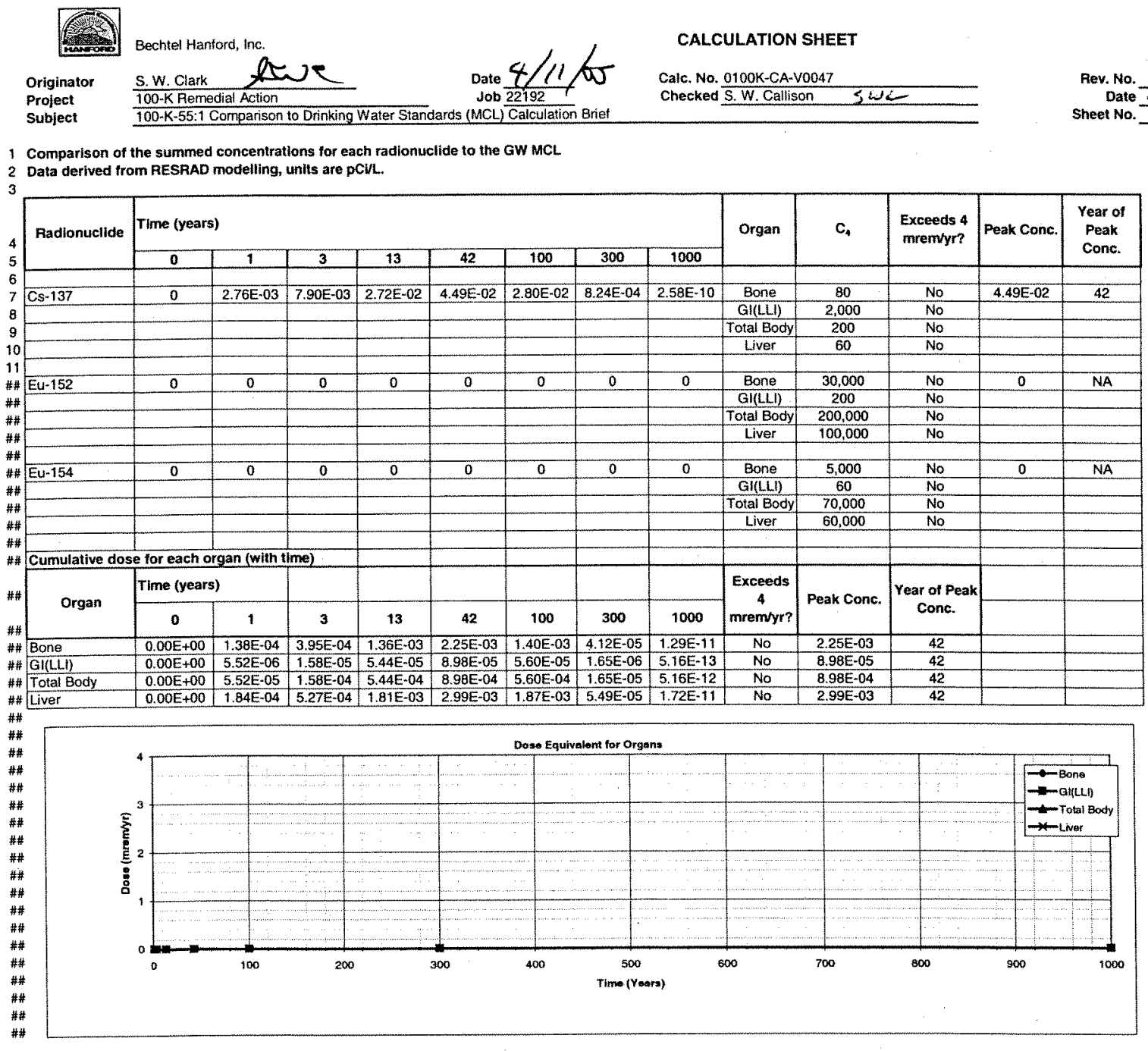




\section{CALCULATION COVER SHEET}

Area

Discipline

Subject

Computer Program
Project Title:

\begin{tabular}{|c|c|c|c|}
\hline \multirow{2}{*}{\multicolumn{2}{|c|}{$\frac{100-\mathrm{K} \text { Remedial Action }}{100-\mathrm{K}}$}} & \multirow[t]{2}{*}{ Job No. } & 22192 \\
\hline & & & \\
\hline Environmental & *Calc. No. & $0100 \mathrm{~K}-\mathrm{CA}-\mathrm{V} 0052$ & \\
\hline 100-K-56:1 Pipeline Variance Calculation & & & \\
\hline
\end{tabular}

The attached calculations have been generated to document compliance with established cleanup levels. These documents should be used in conjunction with other relevant documents in the administrative record.

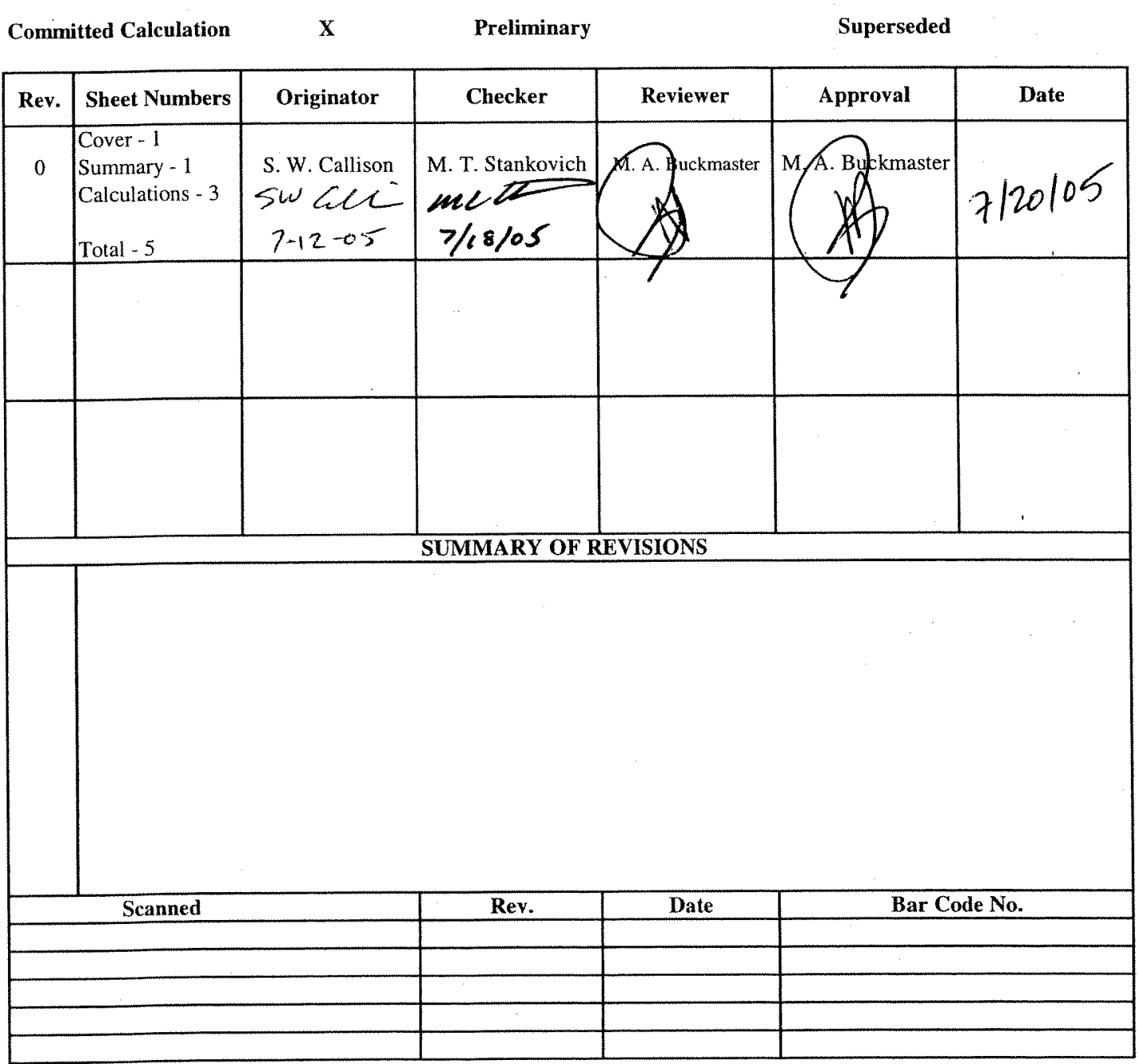

* Obtain Calc. No. from DIS.

DE01437.03 (12/09/2004) 
CVP-2005-00006

Rev. 0

\section{CALCULATION SHEET}

Har Bechtel Hanford Inc.

Originator S.W. Callison Sw C bate

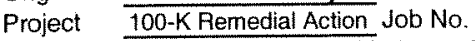

$7 / 12 / 2005$ Calc. No.

Subject 100-K-56:1 Pipeline Variance Calculation

Rev. No.

Date

$7 / 10 / 0$

Conclusion:

2 The required number of samples calculated ( 1 or 2 sample) for each decision sub-unit is less than the default 3 number (4 samples) specified in the DOE/RL-96-22, Rev 4 . Therefore, the default number of samples will be

4 collected from each shallow zone decision sub-unit. 5

6 Problem:

7 Calculate the number of close out samples required for 100-K-56:1 Pipeline Shallow Unit verification sampling 8 as required in "100 Area Remedial Action Sampling and Analysis Plan" (DOE/RL-96-22, Rev 4) and "Instruction 9 Guide for the Remediation of 100 Areas Waste Sites" (0100X-IG-G0001, Rev 5).

1 Given:

12 1) Sample locations for the 100-K-56:1 Pipeline Decision Unit are identified on the 100-K-56:1 Pipelines 13 Shallow Sample Design, Calculation number 0100K-CA-V0053, Rev. 0.

14 2) Lookup values from DOE/RL-96-22, Rev $4 . \quad$ Sw2 7-20-45

15 3) Sample Design requirements from DOE/RL-96-22, Rev 4 and 0100X-IG-G0001, Rev 5.

16 4) Field sampling intormation from sampling logbook EL-1572-2 and EL-1572-3.

18 Solution:

19 Calculation methodology is described in Appendix A of DOE/RL-96-22, Rev 4. Data from attached worksheets

20 are used to calculate the required number of closeout samples. Variance calculation is based on the same

21 three isotopes used to develop the statistical approach in DOE/RL-96-22, Rev 4. The statistical design is

22 based on the premise that these isotopes are the predominant components of the contamination and are

23 representative of the contamination distribution.

24

25 Sheet No. Contents Topic

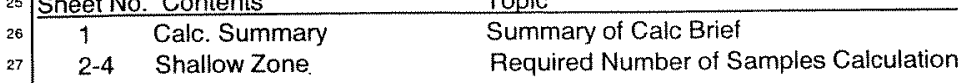

Calc. Summary 


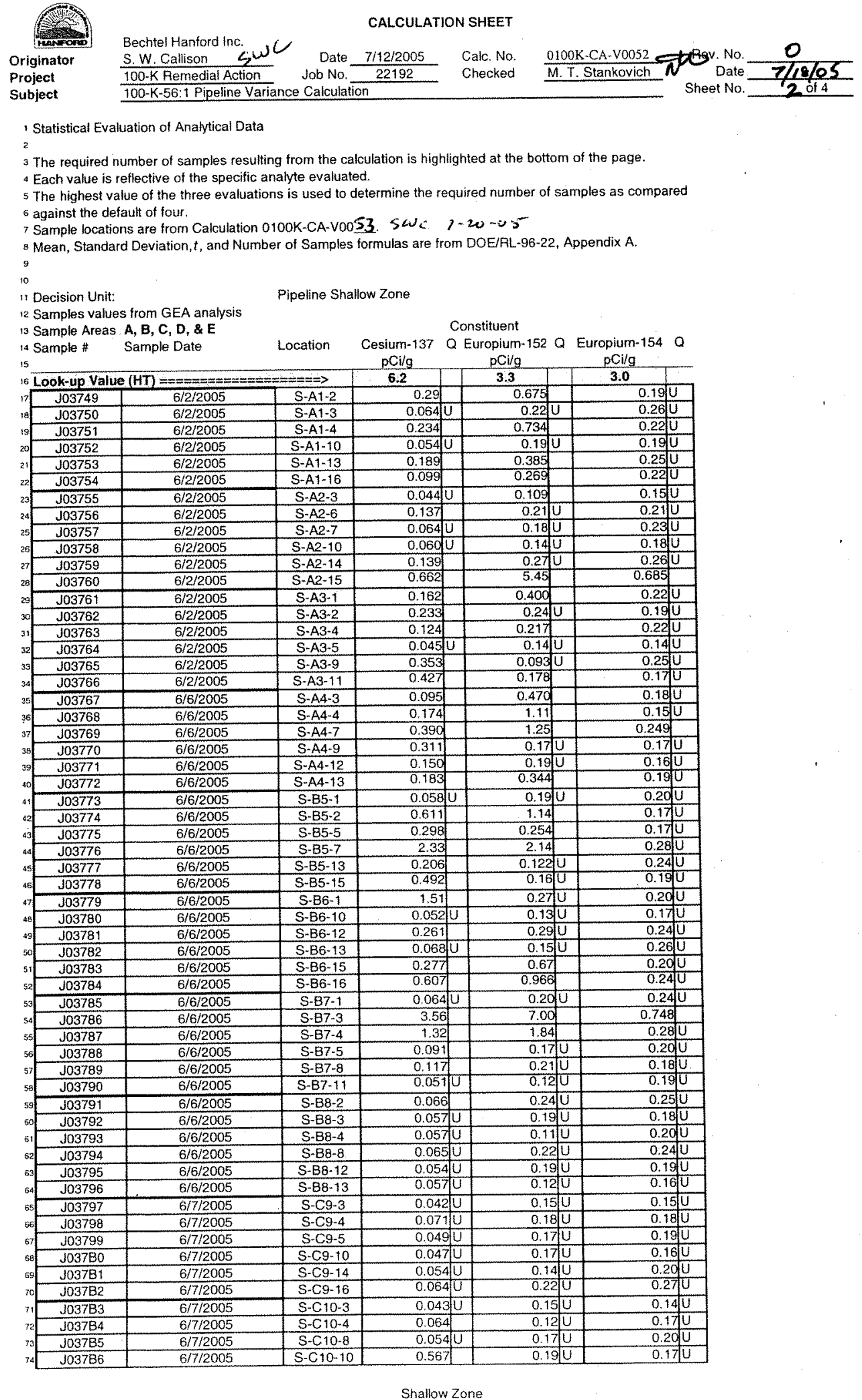




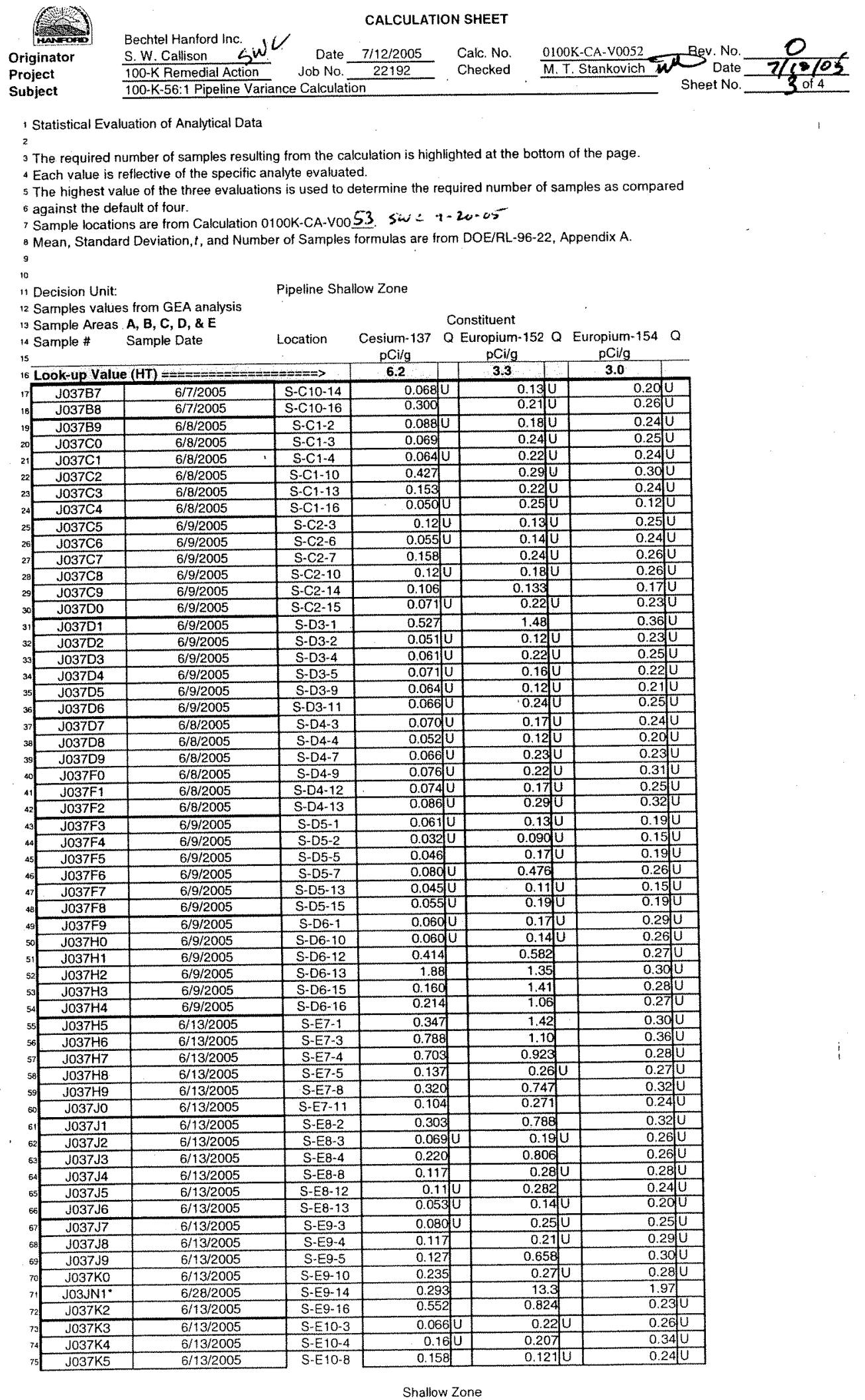


CVP-2005-00006

Rev. 0

CALCULATION SHEET

Originator
Project

\begin{tabular}{|c|c|c|c|c|c|c|}
\hline \multirow{2}{*}{$\begin{array}{l}\text { Bechtel Hanford Inc. } W U \\
\text { S. W. Callison s }\end{array}$} & \multicolumn{3}{|c|}{ CALCULATION SHEET } & \multirow{3}{*}{\multicolumn{2}{|c|}{$\frac{0100 \mathrm{~K}-\mathrm{CA}-\mathrm{V} 00 \mathrm{z}}{\text { M.T.Stankovich } \mathrm{Ul}}$}} & \\
\hline & Date & $7 / 12 / 2005$ & Calc. No. & & & 0 \\
\hline 100-K Remedial Action & Job No. & 22192 & Checked & & & \\
\hline
\end{tabular}

1 Statistical Evaluation of Analytical Data

The required number of samples resulting from the calculation is highlighted at the bottom of the page.

${ }^{4}$ Each value is reflective of the specific analyte evaluated.

5 The highest value of the three evaluations is used to determine the required number of samples as compared

6 against the default of four.

7 Sample locations are from Calculation 0100K-CA-VOO $\$ 3$ swc 7-20-to

* Mean, Standard Deviation, $i$, and Number of Samples formulas are from DOE/RL-96-22, Appendix A.

9

1 Decision Unit:

12 Samples values from $G E A$ analysis

13 Sample Areas A, B, C, D, \& E

14 Sample \# Sample Date

Pipeline Shallow Zone

Constituent

Q

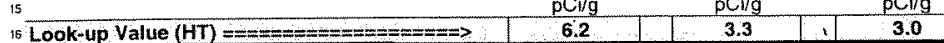

\begin{tabular}{|c|c|c|c|c|c|c|c|}
\hline \multicolumn{3}{|c|}{ 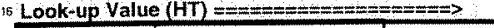 } & 6.2 & & & \multirow{2}{*}{\multicolumn{2}{|c|}{0.270}} \\
\hline J037K6 & $6 / 13 / 2005$ & S-E10-10 & 0.465 & 0.26 & $\mathrm{U}$ & & \\
\hline J037K 7 & $6 / 13 / 2005$ & S.E10-14 & 0.341 & 0.347 & & 0.26 & \\
\hline J037K8 & $6 / 13 / 2005$ & S.E10-16 & 0.083 & 0.121 & & 0.20 & U \\
\hline \multirow{2}{*}{\multicolumn{3}{|c|}{ Mean (LV) $=-x=\ldots$}} & 0.26 & 0.52 & & 0.25 & \\
\hline & & & 0.48 & 1.35 & & 0.19 & \\
\hline \multicolumn{3}{|c|}{$\alpha(5 \%)=m=0==$} & 1.645 & 1.645 & & 1.645 & \\
\hline \multicolumn{3}{|c|}{ 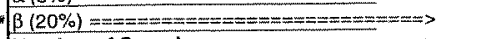 } & 0.842 & 0.842 & & 0.842 & \\
\hline \multicolumn{3}{|c|}{ 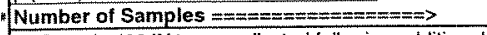 } & 1 & 2 & & 1 & \\
\hline
\end{tabular}


CVP-2005-00006

Rev. 0

C-108 


\section{CALCULATION COVER SHEET}

(Sample Design, Verification, or Waste Characterization Calculation)

Project Title:

\section{Area}

Discipline

Subject

Computer Program

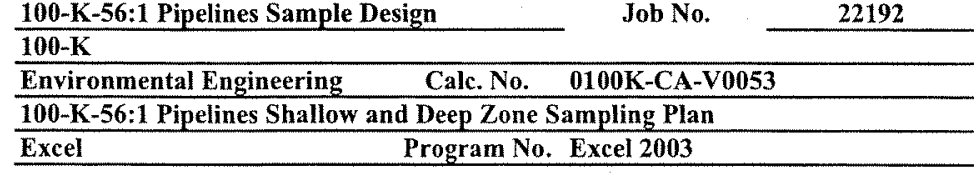

The attached calculations have been generated to document compliance with established cleanup levels. These documents should be used in conjuction with other relevent documents in the administrative record.

$\begin{array}{llll}\text { Committed Calculation } & \text { Preliminary } & \square & \text { Superseded } \\ \end{array}$

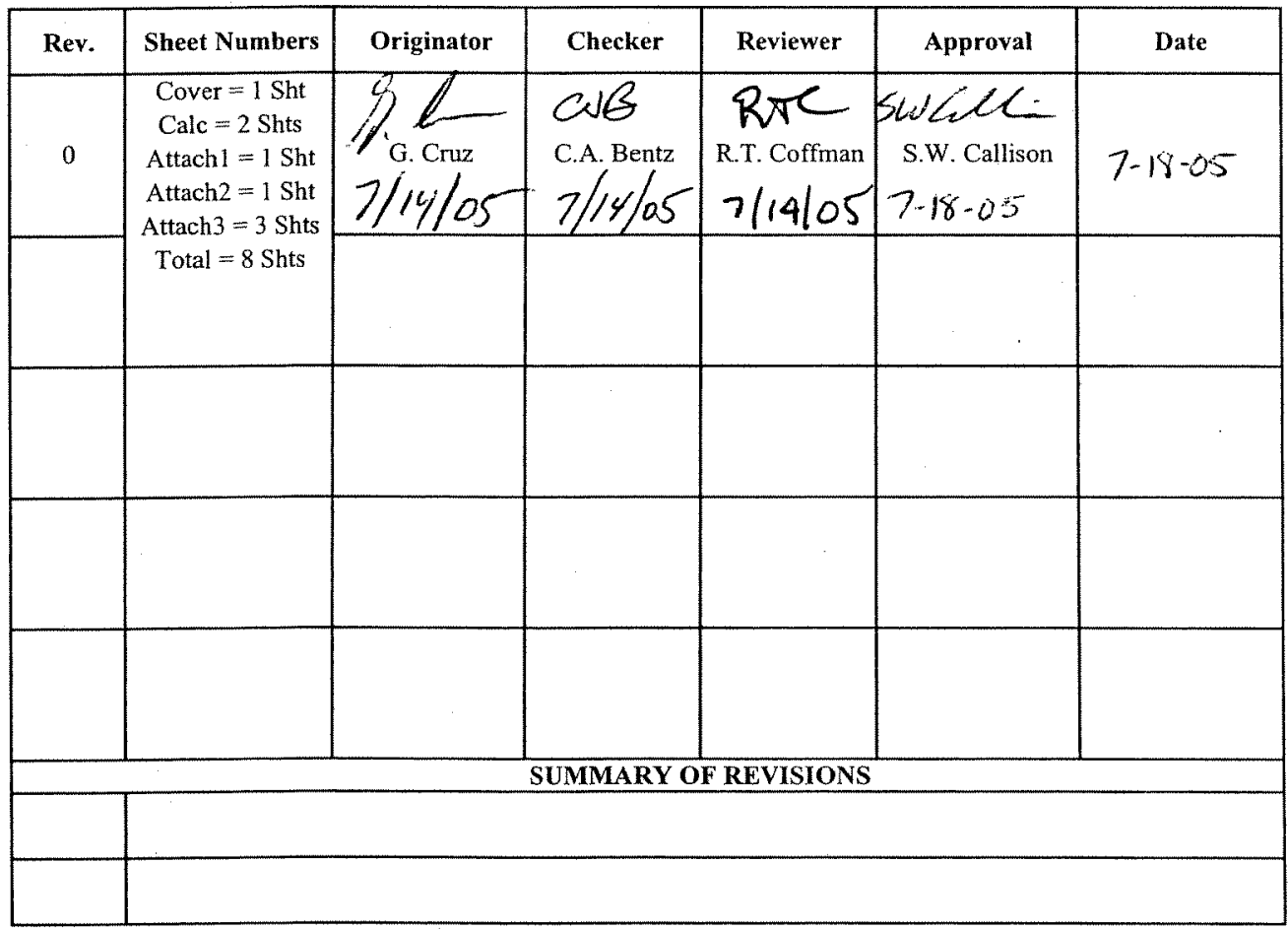

* Obtain Calc. No, from DIS

January 2003

DE01-437.03 
Date $7 / 13 / 2005$

Project 100-K-56:1 Pipelines Sample Design

Subject
Calc. No. $0100 \mathrm{~K}-\mathrm{CA}-\mathrm{V} 0053$

Job No. 22192 Checked
Rev. No. 0 QsB Date $7 / 14 / 05$ Sheet No. 1 of 2

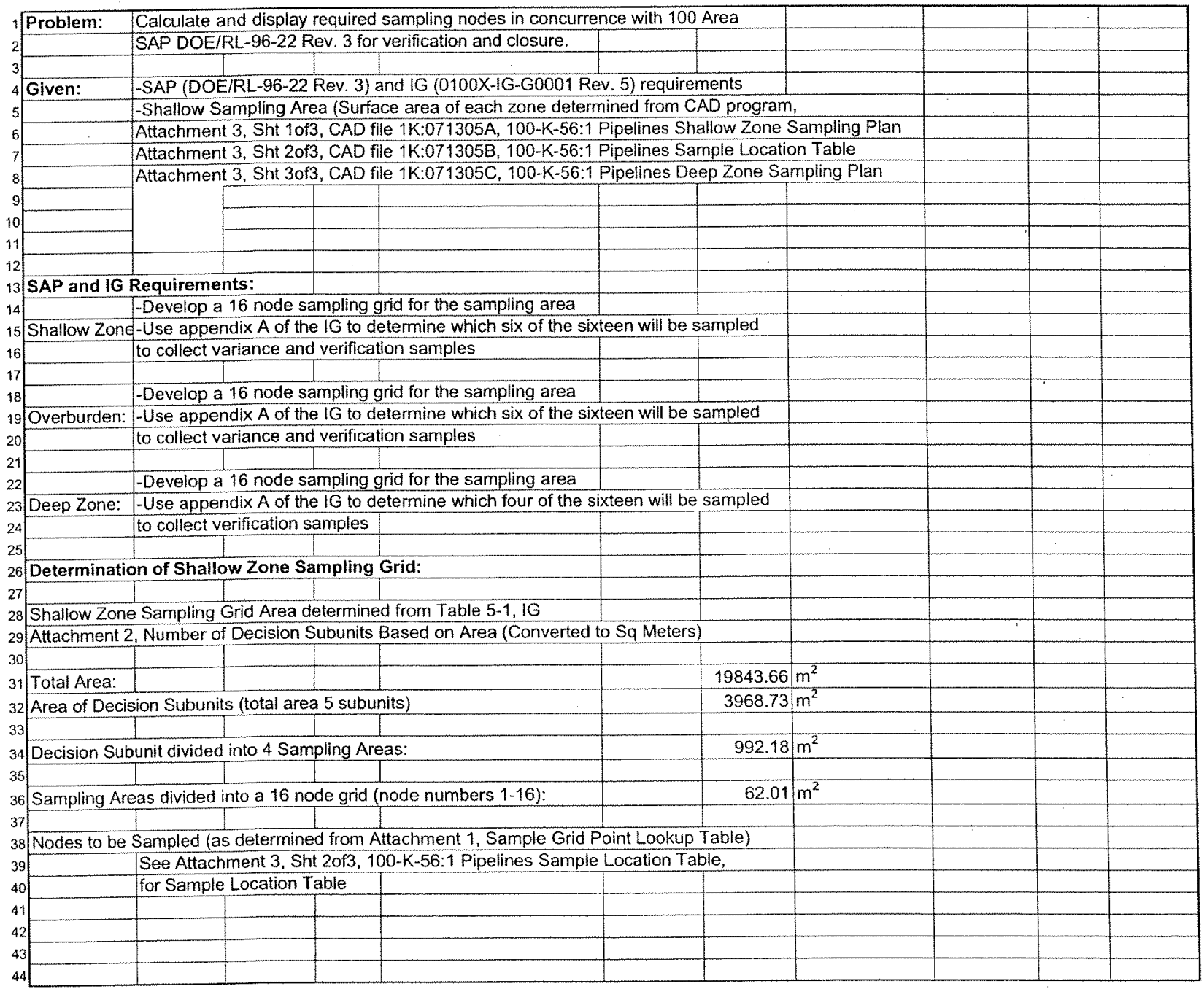


Date $7 / 13 / 2005$

Project $\quad$ 100-K-56:1 Pipelines Sample Design

Subject

100-K-56:1 Pipelines Shallow and Dee
Calc. No. 0100K-CA-V0053

Job No. 22192 Checked
Rev. No. 0 cos Date $7 / 14 / 05$ Sheet No. 2 of 2

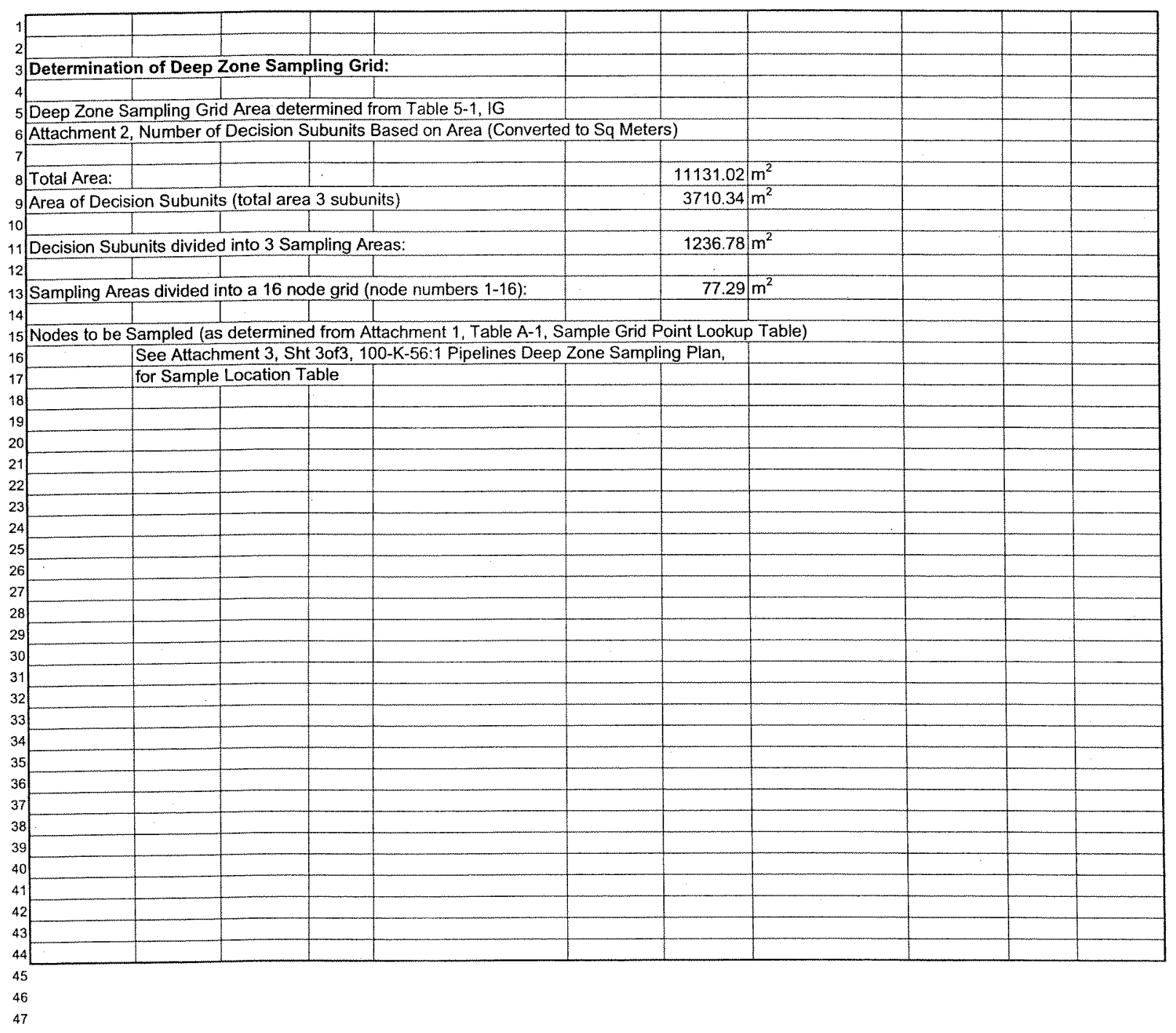


CVP-2005-00006

Rev. 0

Bechtef Hanford, Jnc.

runions

Originator

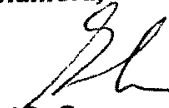

Date

$7 / 13 / 2005$

Calc. No.0100K-CA-V0053

Rev. No.0

Project 100-K-56:1 Pipelines Sample Design Job No. 22192 Checked csB Date $7 / 14 / 05$

Subject 100-K-56:1 Pipelines Shallow and Deep Zone Sampling Plan Sheet No 1 of 1

\footnotetext{
4
}

, ATTACHMENT 1 ${ }_{3}$ Sample Grid Point Lookup Table.

\begin{tabular}{|c|c|c|c|c|c|c|c|c|c|c|}
\hline Default Plan & $\begin{array}{c}\text { Sampling } \\
\text { Area } 1\end{array}$ & $\begin{array}{l}\text { Sampling } \\
\text { Area } 2\end{array}$ & $\begin{array}{l}\text { Sampling } \\
\text { Area } 3\end{array}$ & $\begin{array}{c}\text { Sampling } \\
\text { Area } 4\end{array}$ & $\begin{array}{c}\text { Sampling } \\
\text { Area } 5\end{array}$ & $\begin{array}{c}\text { Sampling } \\
\text { Area } 6\end{array}$ & $\begin{array}{c}\text { Sampling } \\
\text { Area } 7\end{array}$ & $\begin{array}{c}\text { Sampling } \\
\text { Area } 8\end{array}$ & $\begin{array}{c}\text { Sampling } \\
\text { Area } 9\end{array}$ & $\begin{array}{c}\text { Sampling } \\
\text { Area } 10\end{array}$ \\
\hline VarianceNerification & 3 & 6 & 1 & 4 & 5 & 1 & 3 & 3 & 4 & 16 \\
\hline Variance/Nerification & 4 & 7 & 11 & 3 & 15 & 15 & 5 & 13 & 10 & 10 \\
\hline VarianceNerification & 16 & 3 & 2 & 7 & 7 & 10 & 11 & 4 & 3 & 14 \\
\hline Variance Nerification & 10 & 15 & 4 & 12 & 1 & 13 & 4 & 8 & 16 & 4 \\
\hline Variance & 2 & 14 & 5 & 9 & 13 & 12 & 8 & 2 & 14 & 8 \\
\hline Variance & 13 & 10 & 9 & 13 & 2 & 16 & 1 & 12 & 5 & 3 \\
\hline Not Sampling & 6 & 1 & 10 & 8 & 14 & 4 & 16 & 5 & 8 & 6 \\
\hline Not Sampling & 1 & 9 & 13 & 1 & 10 & 5 & 12 & 1 & 1 & 15 \\
\hline Not Sampling & 9 & 12 & 7 & 5 & 6 & 2 & 6 & 7 & 15 & 9 \\
\hline Not Sampling & 15 & 16 & 15 & 14 & 16 & 6 & 2 & 15 & 11 & 1 \\
\hline Not Sampling & 8 & 13 & 8 & 10 & 12 & 11 & 13 & 14 & 2 & 12 \\
\hline Not Sarnpling & 5 & 2 & 3 & 11 & 4 & 3 & 9 & 10 & 7 & 11 \\
\hline Nat Sampling & 7 & 11 & 14 & 15 & 11 & 14 & 14 & 6 & 13 & 2 \\
\hline Not Sampling & 11 & 4 & 6 & 2 & 9 & 7 & 7 & 11 & 9 & 7 \\
\hline Not Sampling & 12 & 8 & 16 & 16 & 3 & 8 & 15 & 9 & 6 & 13 \\
\hline Not Sampling & 14 & 5 & 12 & 6 & 8 & 9 & 10 & 16 & 12 & 5 \\
\hline
\end{tabular}

Not Sampling

** Note: Grid nodes for each sampling area in each waste site should be numbered consistently, e.g., begin numbering

24 the nodes in the northwestern-most node, then number consecutively left to right. 
CVP-2005-00006

Rev. 0

Bechtel/Hanford, Inc.

than

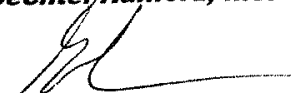

Originator $\mathrm{G}$. Cruz

Date $7 / 13 / 2005$

Calc. No. 0100K-CA-V0053

Rev. No. 0

Project 100-K-56:1 Pipelines Sample Design Job No. 22192 Checked CSB Date $7 / 14 / 05$

Subject 100-K-56:1 Pipelines Shallow and Deep Zone Sampling Plan Sheet No. 1 of 1

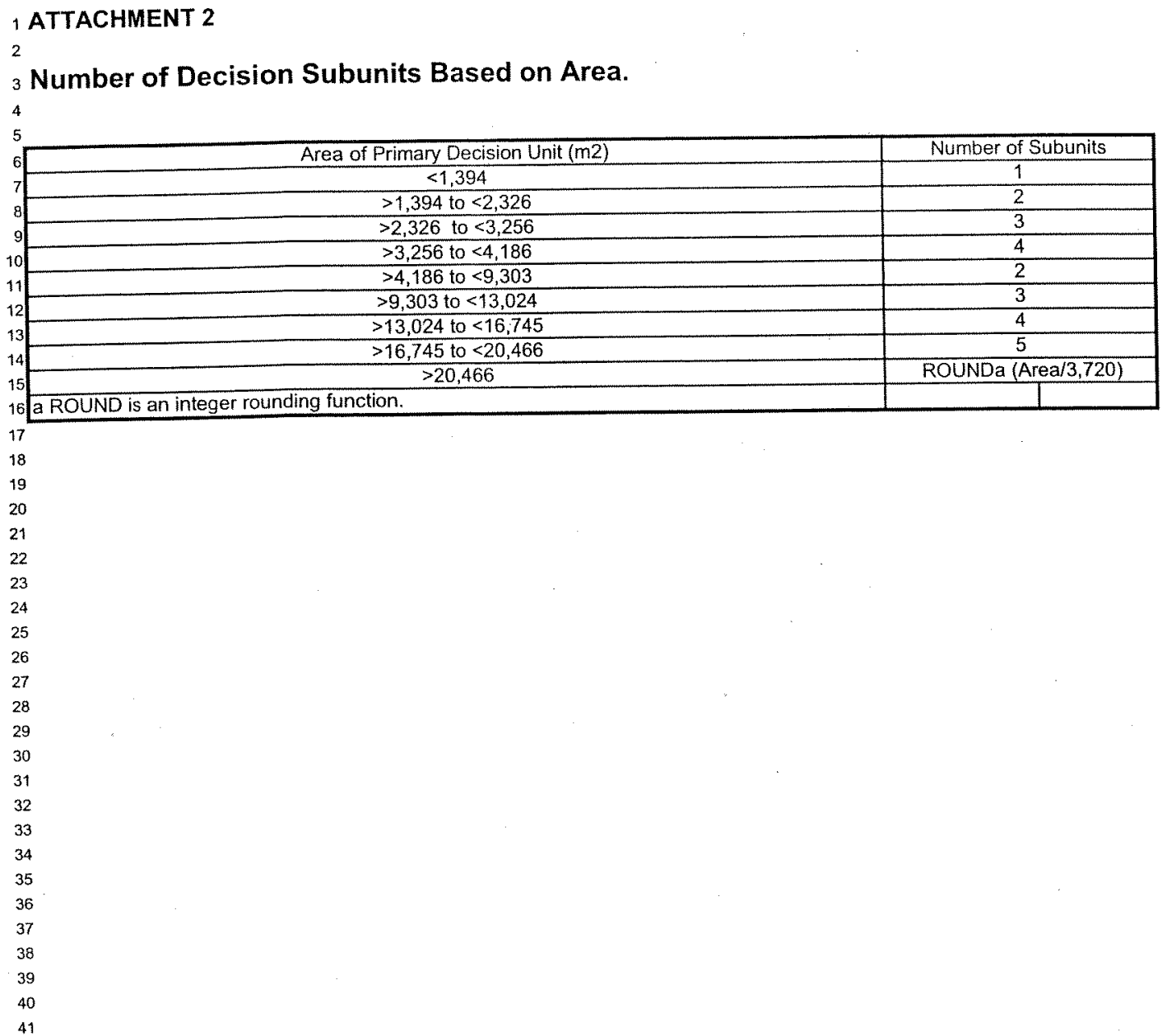


CVP-2005-00006

Rev. 0

C-114 


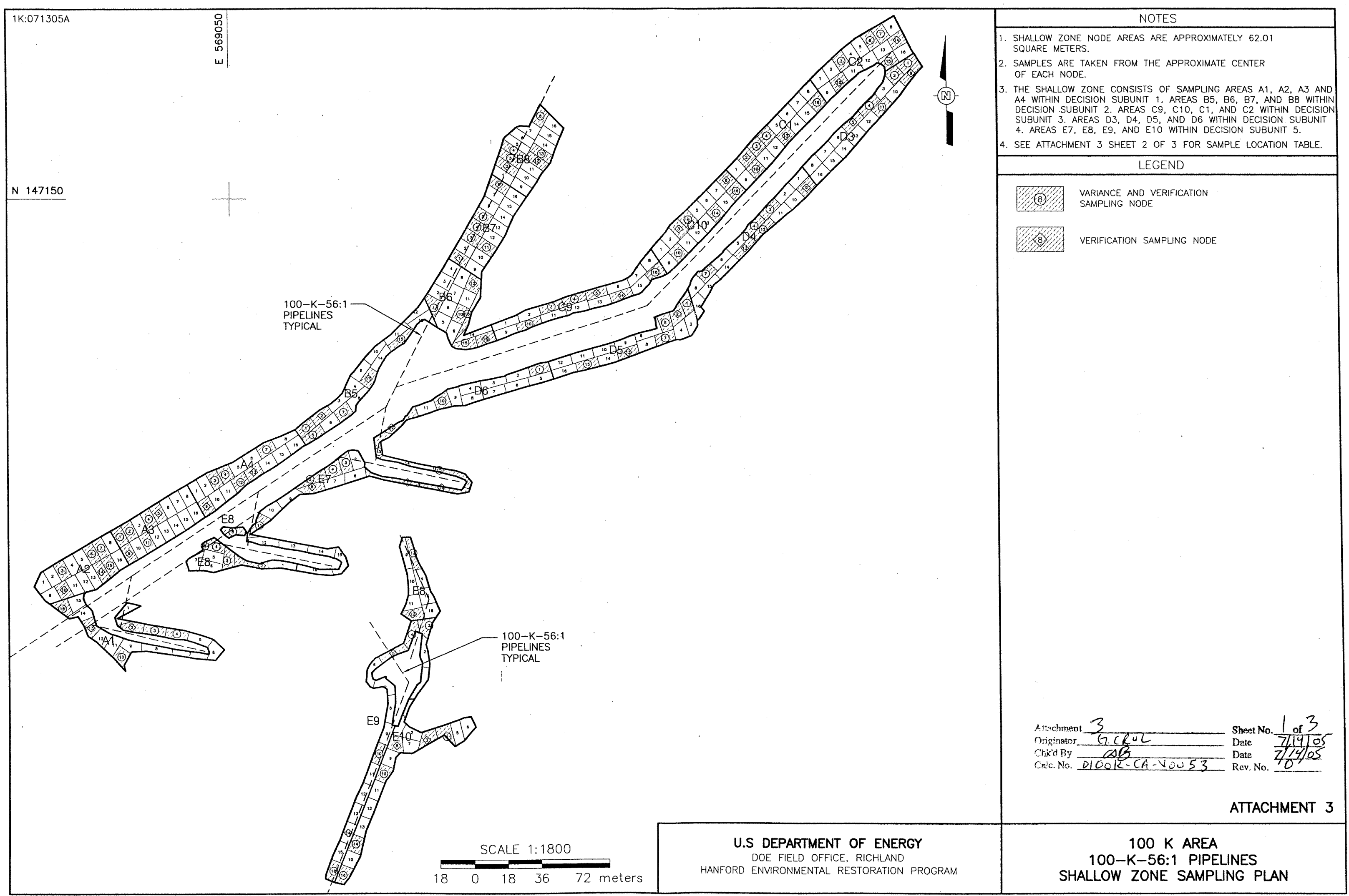



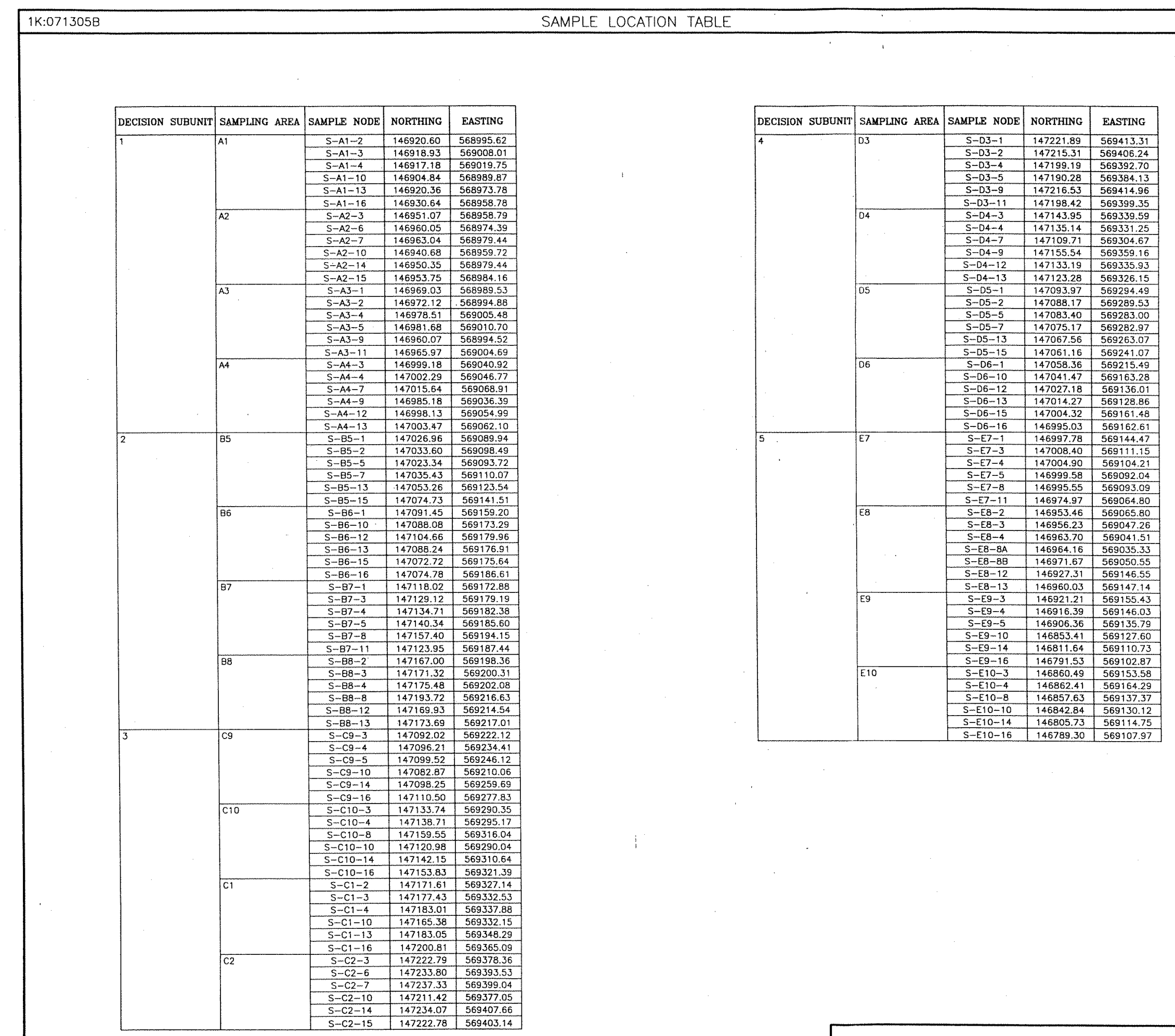

Atrachmen 3

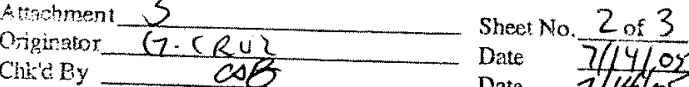

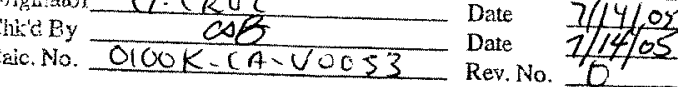

ATTACHMENT 3 


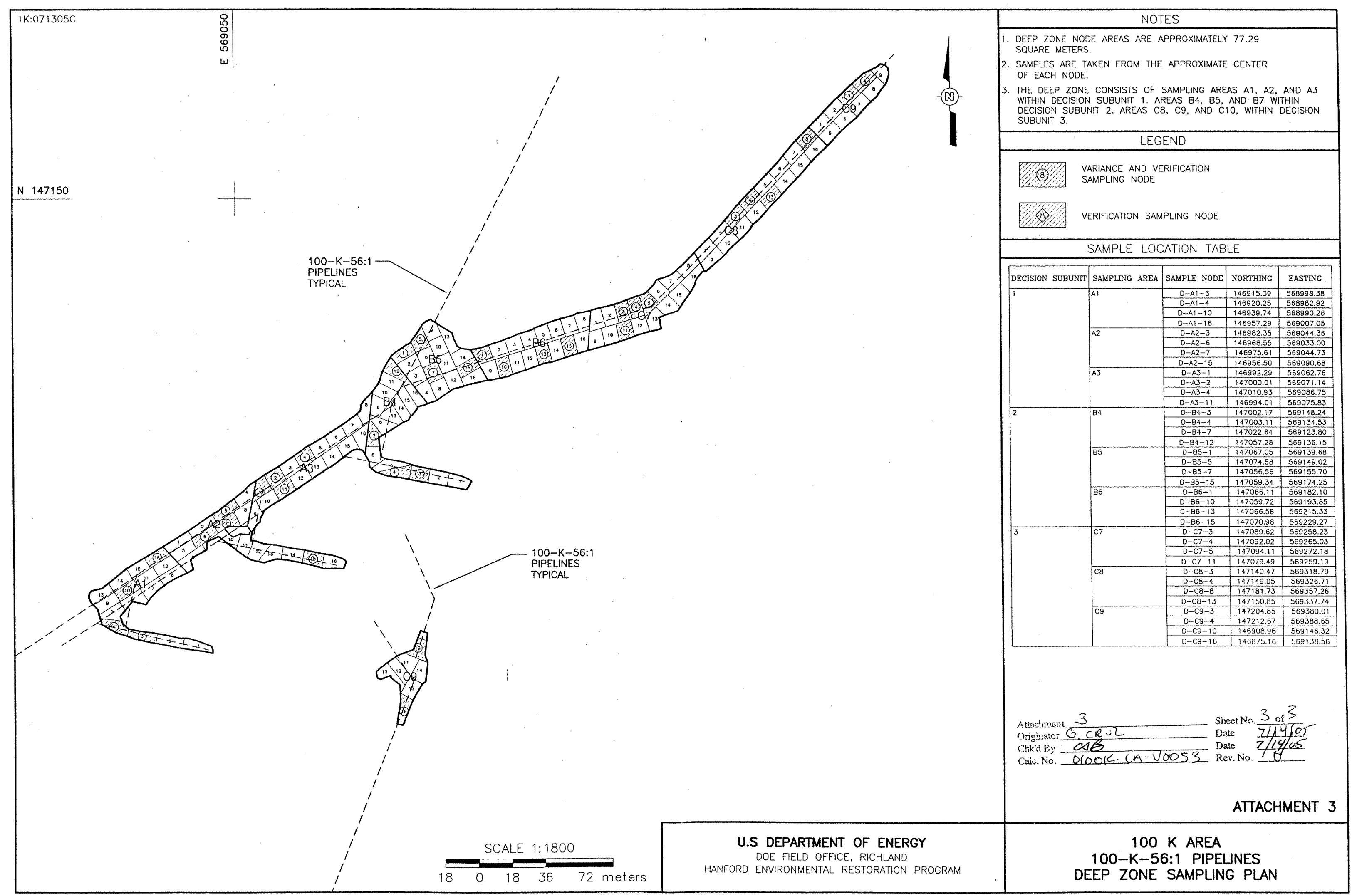




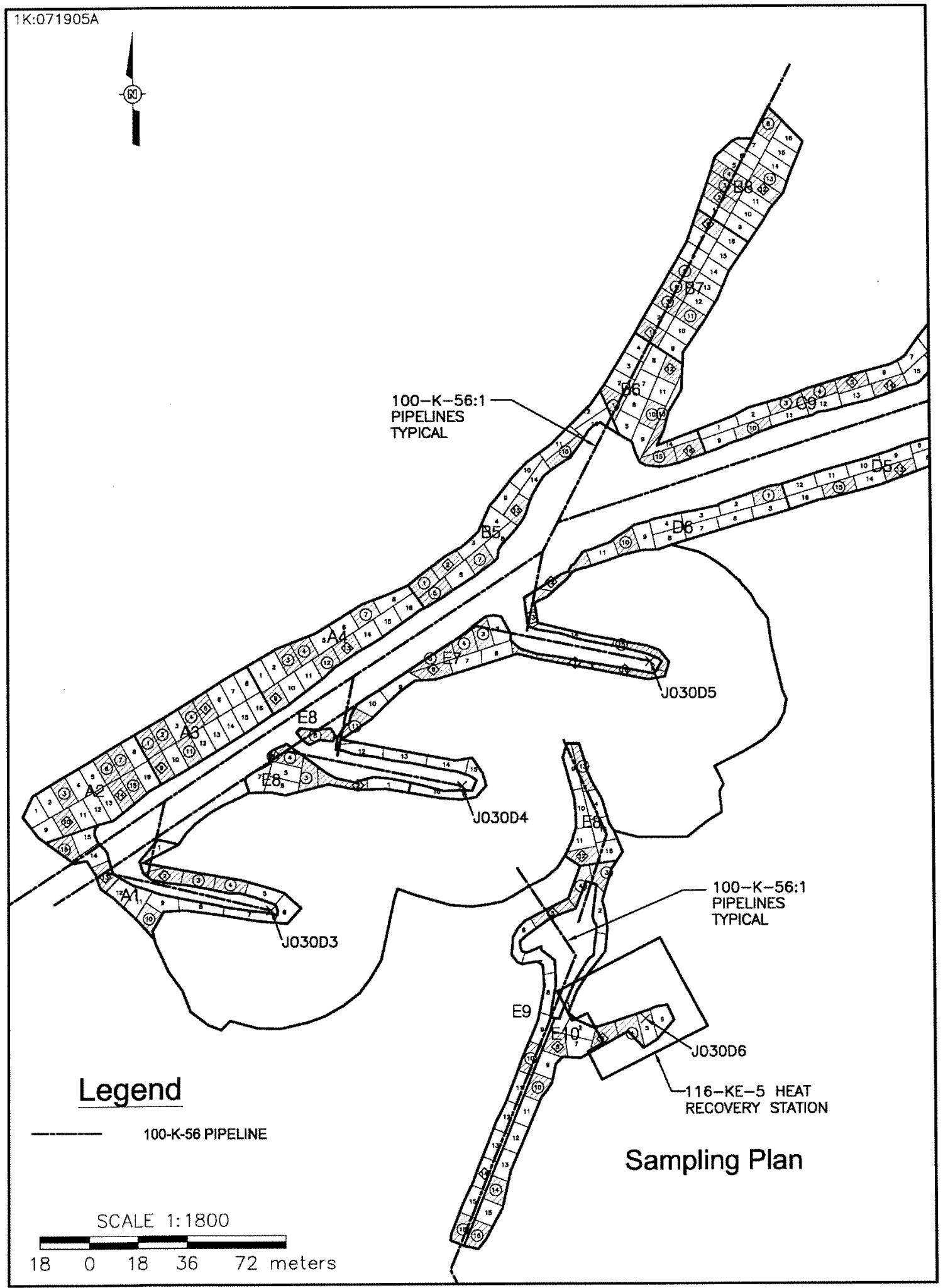


CVP-2005-00006

Rev. 0

C-120 
CVP-2005-00006

Rev. 0

\section{CALCULATION COVER SHEET}

Project Title:

Area

Discipline

Subject

Computer Program
100-K Area Remedial Action

Job No. $100-\mathrm{K}$

Environmental

100-K-56:1 Pipeline Cleanup Verification 95\% UCL Calculation

Excel Program No. Excel 2003

The attached calculations have been generated to document compliance with established cleanup levels. These documents should be used in conjunction with other relevant documents in the administrative record.

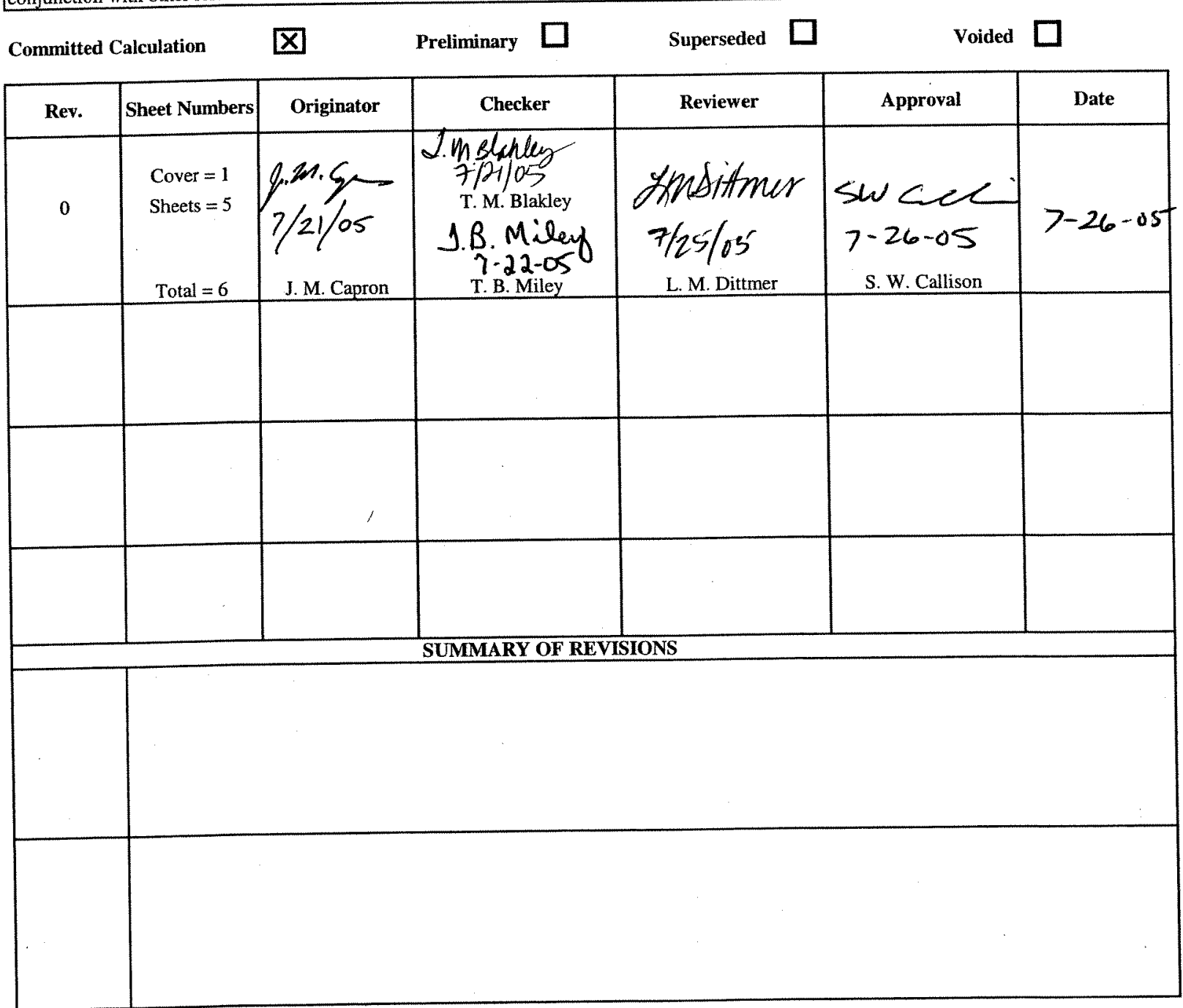

* Obtain cale no. from DIS

DE01437.03 (12/09/2004) 
CVP-2005-00006

Rev. 0

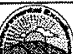

Calc. No. 0100K-CA-V0049

Checked T.M. Blakleyotho

Checked T. B. Miley $\$ 8 M$

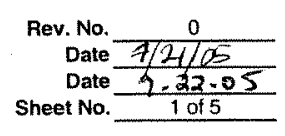

\section{Purpose:}

Calculate the $95 \%$ upper confidence limit (UCL) to evaluate compliance with cleanup standards for the subject site. Also, calculate the carcinogenic risk for applicable nonradionuclide 3 analytes, pertorm the Washington Administrative Code (WAC) 173-340 (Model Toxics Control Act [MTCA]) 3-part test, if required, and calculate the relative percent difference (RPD) 4 analytes, pertorm the Washington Administrative Code (WAC)

15 2) All lookup values and remedial action goals (RAGs) are taken from the Remedial Design Repor/Remedial Action Work Plan (RDR/RAWP) (DOE-RL 2005b) and

16 Ecology (1996) unless otherwise specified.

17 3) DOE-RL, 2005a, 100 Area Remedial Action Sampling and Analysis Plan, DOE/RL-96-22, Rev. 4, U.S. Department of Energy, Richland Operations Office, Richland,

18 Washington.

19 4) DOE-RL, 2005b, Remedial Design Report/Pemedial Action Work Plan for the 100 Area, DOE/RL-96-17. Rev. 5, U.S. Department of Energy, Richland Operations Office,

20 4) DOE-RL, 2005b, Remedia

21 Fichland, Washington.

22 5) Ecology, 1992, Statistical Guldance for Ecology Site Managers, Supplement S-6, Analyzing Site or Background Data with Below-Detection Limit or Below-POL

23 6) Ecology, 1993 , Statistical Guldance (Censored Data Sets). Publication \#92-54, Washington State Department of Ecology, Olympia, Washington.

24 Values (Censored Data Sets), Publication \#92-54, Washington State Department of Ecology, Olympla, Washington.

25 7) Ecology, 1996,

26 Washington.

28 Washington, D.C.

WAC 173-340, 1996, "Model Toxics Control Act--Cleanup," Washington Administrative Cade

Solution:

Calculation methodology is described in Ecology Pub. \#92-54 (Ecology 1992, 1993), below, and in the RDR/RAWP (DOE-RL 2005b). Use data from the attached worksheets to performed by utilizing the bulit-in spreadsheet functions and/or creating formulae within the cells. The statistical evaluation of data for USe in accordance with the RDF/RAWP (DOE-AL

42 Melhodology: 43 The statistical value calculated to evaluate the effectiveness of cleanup was the $95 \%$ uch. For no being below detection limits are set to $1 / 2$ the detection limit value for calculation of the

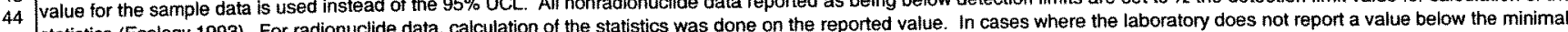
45 statistics (Ecology 1993). For radionuclide data, calculation of the statistics was done on the reported duplicate sample pairs, the samples are averaged before being included in the data delectable acliny (NDA), hallof the mata as described above.

47 set,

48

49 For nonradionuclides, the WAC $173-340$ statistical guidance suggests that a test for distributional form be performed on the data and the $95 \%$ UCL calculated on the appropriate 50 distribution using Ecology software. For nonradionuclide small data sets $(n<10)$ and all radionuclide data sets, the calculations are performed assuming nonparametric distribution, so 1 dest for distribution is performed. For nonradionuclide data sets of ten or greater, distributional testing is done using Ecology's MTCAStat software (Ecology 1993). Note that no 52 nonradionuclide COCs have been identified for the 100-K-56:1 pipelines.

53

The estimated hazard quotient (for applicable nonradionuclide COCs) is determined by dividing the statistical value (derived in this calculation) by the WAC $173-340$ non carcinogenic 55 . estim limit.

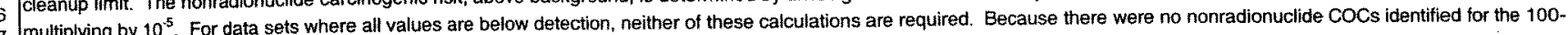
K-56:1 pipelines, neither of these calculations was performed.

58

The WAC 173-340 3-part test is performed for nonradionuclide analytes only and determines if

61 1) the statistical value exceeds the most stringent cleanup limit for each non-radionuclide COC

62 2) greater than $10 \%$ of the raw data exceed the most stringent cleanup limit for each non-radionuclide COC,

63 2) gre maximum value of the raw data set exceeds two times the most stringent cleanup limit for each non-radionuclide $\mathrm{COC}$

64

RPD is calculated when both the main value and either the duplicate or split values are above detection limits and are greater than 5 times the target detection limit (TDL). The

The

2005a). The APD calculations use the following formula: $\mathrm{RPD}=[\mathrm{M}-\mathrm{S} V((\mathrm{M}+\mathrm{S}) / 2)]^{*} 100$

\section{$S=$ Split (or duplicate) Sample Vatue} 政 discussion as necessary is provided in the data quality assessment section of the applicable CVP.

75 
CVP-2005-00006

Rev. 0

\section{CALCULATION SHEET}

Haw ooro Bechtel Hantord, lne

Date 07/21/05 Job No. 22192

\section{Project 100 -K Area Regredial Action \\ Subject $100-\mathrm{K}-56: 1$ Ppeline Cleanup Verification $95 \%$ UCL Calculation}

Summary (continued)

\section{Results:}

2 The results presented in the summary tables that follow are for use in RESAAD dose/risk analysis and the CVP for this site

\begin{tabular}{|c|c|c|c|}
\hline \multicolumn{4}{|c|}{ Results Summary - Shallow Zone } \\
\hline Analyte & Result & Qualifier & Units \\
\hline Cesium-137 & $6.1 \mathrm{E}-01$ & & $\mathrm{pCi} / \mathrm{g}$ \\
\hline Europium-152 & $1.7 E+00$ & & $\mathrm{pCl} / \mathrm{g}$ \\
\hline Europium-154 & $1.4 \mathrm{E}-01$ & U & $\mathrm{pCi} / \mathrm{g}$ \\
\hline
\end{tabular}

10 Relative Percent Difference (RPD)

11 Results (Shallow Zone)* QAOC Analysis

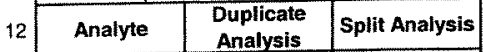

$1 3 \longdiv { \text { Cesium-137 } }$

14 Europium-152

15 Europium-154

16 "A blank cell indicates that RPD evaluation was not required.

$17 \mathrm{CVP}$ = cleanup verification package

$18 O A O C=$ quality assurance/quality control

19 RESPAD = RESidual RADioactivity (dose model)

$20 \mathrm{U}=$ undetected

\begin{tabular}{|c|c|c|c|}
\hline \multicolumn{4}{|c|}{ Results Summary - Deep Zone } \\
\hline Analyte & Result & Qualitier & Units \\
\hline Cesium-137 & $1.32 \mathrm{E}+01$ & & $\mathrm{pCl} / \mathrm{g}$ \\
\hline Europium-152 & $1.37 \mathrm{E}+01$ & & $\mathrm{pCi} / \mathrm{g}$ \\
\hline Europium-154 & $1.87 \mathrm{E}+00$ & & $\mathrm{pCi} / \mathrm{g}$ \\
\hline
\end{tabular}

\begin{tabular}{|c|c|c|}
\hline \multicolumn{3}{|c|}{$\begin{array}{r}\text { Relative Percent Difference (APD) } \\
\text { Results (Deep Zone)* OA/OC Analysis }\end{array}$} \\
\hline Analyte & $\begin{array}{c}\text { Duplicate } \\
\text { Analysis }\end{array}$ & Split Analysis \\
\hline Cesium-137 & & $47.5 \%$ \\
\hline Europium-152 & & $51.3 \%$ \\
\hline Europium-154 & & \\
\hline
\end{tabular}

Rev. No.

Calc. No. $0100 \mathrm{~K}$-CA-V0049

Checked T. M. Blakley $\mathrm{m} / 3$

Checked T. B. Miley JBM
Date

$7 / 24$ th

Date $\{-22-05$ 2 o1 


\section{(itim 11. Bechtel Hanford, Inc.}

Originator J.M. Capron $\& \ln C$ Project 100-K Area Rentedial Action Subject 100-K-56:1 Pipeline Cleanup Verification 95\% UCL Calculation

Job No. 22192

Shallow Zone Sample Data

1 Shallow Zone Sample Data

\begin{tabular}{|c|c|c}
\hline $\begin{array}{c}\text { Sample } \\
\text { Date }\end{array}$ \\
$6 / 23 / 2005$ \\
$6 / 23 / 2005$ \\
\hline $6 / 23 / 2005$ \\
\hline $6 / 23 / 2005$ \\
\hline $6 / 23 / 2005$ \\
\hline $6 / 23 / 2005$ \\
\hline $6 / 23 / 2005$ \\
\hline $6 / 23 / 2005$ \\
\hline $6 / 23 / 2005$ \\
\hline $6 / 23 / 2005$ \\
\hline $6 / 23 / 2005$ \\
\hline $6 / 23 / 2005$ \\
\hline $6 / 23 / 2005$ \\
\hline $6 / 23 / 2005$ \\
\hline $6 / 23 / 2005$ \\
\hline $6 / 23 / 2005$ \\
\hline $6 / 23 / 2005$ \\
\hline $6 / 23 / 2005$ \\
\hline $6 / 23 / 2005$ \\
\hline $6 / 23 / 2005$ \\
\hline $6 / 23 / 2005$ \\
\hline
\end{tabular}

\begin{tabular}{|c|c|c|c|}
\hline \multicolumn{3}{|c|}{ Cesium-137 } \\
\hline $\mathrm{pCl} / \mathrm{g}$ & $\mathrm{Q}$ & MDA & \\
\hline $1.21 \mathrm{E}-01$ & & $4.6 \mathrm{E}-02$ & 3.42 \\
\hline
\end{tabular}

Calc. No. 0100K-CA-V0049 Checked T.M. Blaklew ma/s Checked T. B. Miley $1 B \mathrm{BM}$

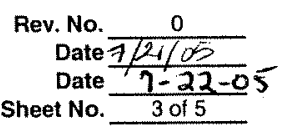

Europium-154

\begin{tabular}{|c|c|c|c|c|c|}
\hline \multicolumn{3}{|c|}{ Europium-152 } & \multicolumn{3}{|c|}{ Europium-154 } \\
\hline $\mathrm{pCi} / \mathrm{g}$ & $Q$ & MDA & $\mathrm{pCi} / \mathrm{g}$ & $\mathbf{Q}$ & MDA \\
\hline $42 \mathrm{E}-01$ & & $7.0 \mathrm{E}-02$ & $1.3 \mathrm{E}-01$ & $\bar{U}$ & $1.3 E-01$ \\
\hline $89 E-01$ & & 8.9E-02 & $1.6 \mathrm{E}-01$ & $\mathrm{U}$ & $1.6 \mathrm{E}-01$ \\
\hline $41 E+00$ & & $1.2 \mathrm{E}-01$ & $2.2 \mathrm{E}-01$ & $\mathbf{U}$ & $2.2 \mathrm{E}-01$ \\
\hline $10 \mathrm{E}-01$ & & 1.0E-01 & $1.7 \mathrm{E}-01$ & $\mathrm{U}$ & $1.7 E-01$ \\
\hline $.5 \mathrm{E}-01$ & $\mathrm{U}$ & $1.5 \mathrm{E}-0 \mathrm{t}$ & $1.1 \mathrm{E}-0 t$ & $\underline{U}$ & $1.1 \mathrm{E}-01$ \\
\hline $48 \mathrm{E}+00$ & & $1.3 \mathrm{E}-01$ & $2.4 \mathrm{E}-01$ & $\bar{U}$ & $2.4 \mathrm{E}-01$ \\
\hline $.3 E-01$ & $\bar{U}$ & 1.3E-01 & $1.0 \mathrm{E}-01$ & $\mathrm{U}$ & $1.0 \mathrm{E}-01$ \\
\hline $17 E+00$ & & 1.1E-01 & 4.7E-01 & $U$ & $4.7 \mathrm{E}-01$ \\
\hline $4 \mathrm{E}-02$ & $\bar{U}$ & $9.4 E-02$ & $1.3 \mathrm{E}-01$ & $U$ & $1.3 \mathrm{E}-01$ \\
\hline IE-01 & $\bar{U}$ & $1.1 \mathrm{E}-01$ & $1.0 \mathrm{E}-01$ & $U$ & $1.0 \mathrm{E}-01$ \\
\hline $7 \mathrm{E}-02$ & $\mathrm{U}$ & $7.7 \mathrm{E}-02$ & $1.0 \mathrm{E}-01$ & $\bar{U}$ & $1.0 \mathrm{E}-01$ \\
\hline $.1 E-01$ & $\bar{U}$ & 1.1E-01 & 1.5E-01 & $U$ & $1.5 \mathrm{E}-01$ \\
\hline $1.1 \mathrm{E}-01$ & $\mathrm{U}$ & $1.1 \mathrm{E}-01$ & $1.0 E-01$ & $\mathrm{U}$ & $1.0 \mathrm{E}-01$ \\
\hline $7.0 \mathrm{E}-02$ & $\bar{U}$ & $7.0 \mathrm{E}-02$ & 1.0E-01 & $\mathrm{U}$ & 1.0E-01 \\
\hline $1.0 \mathrm{E}-01$ & U & $1.0 \mathrm{E}-01$ & $1.2 \mathrm{E}-01$ & U & $1.2 E-01$ \\
\hline $1.1 \mathrm{E}-01$ & $U$ & 1.1E-01 & $1.3 \mathrm{E}-01$ & $U$ & $1.3 \mathrm{E}-01$ \\
\hline $1.6 \mathrm{E}-01$ & $U$ & $1.6 \mathrm{E}-01$ & $1.1 \mathrm{E}-01$ & $\bar{u}$ & $1.1 E-01$ \\
\hline $.04 \mathrm{E}+01$ & & $1.6 \mathrm{E}-01$ & $1.1 \mathrm{E}+00$ & $u$ & $1.1 \mathrm{E}+00$ \\
\hline $42 \mathrm{E}-01$ & & $9.9 \mathrm{E}-02$ & $1.6 \mathrm{E}-01$ & $\mathrm{U}$ & $1.6 \mathrm{E}-01$ \\
\hline$=01$ & & $1.0 \mathrm{E}-01$ & $1.4 \mathrm{E}-01$ & $\mathrm{U}$ & $1.4 \mathrm{E}-01$ \\
\hline & & & & & \\
\hline
\end{tabular}

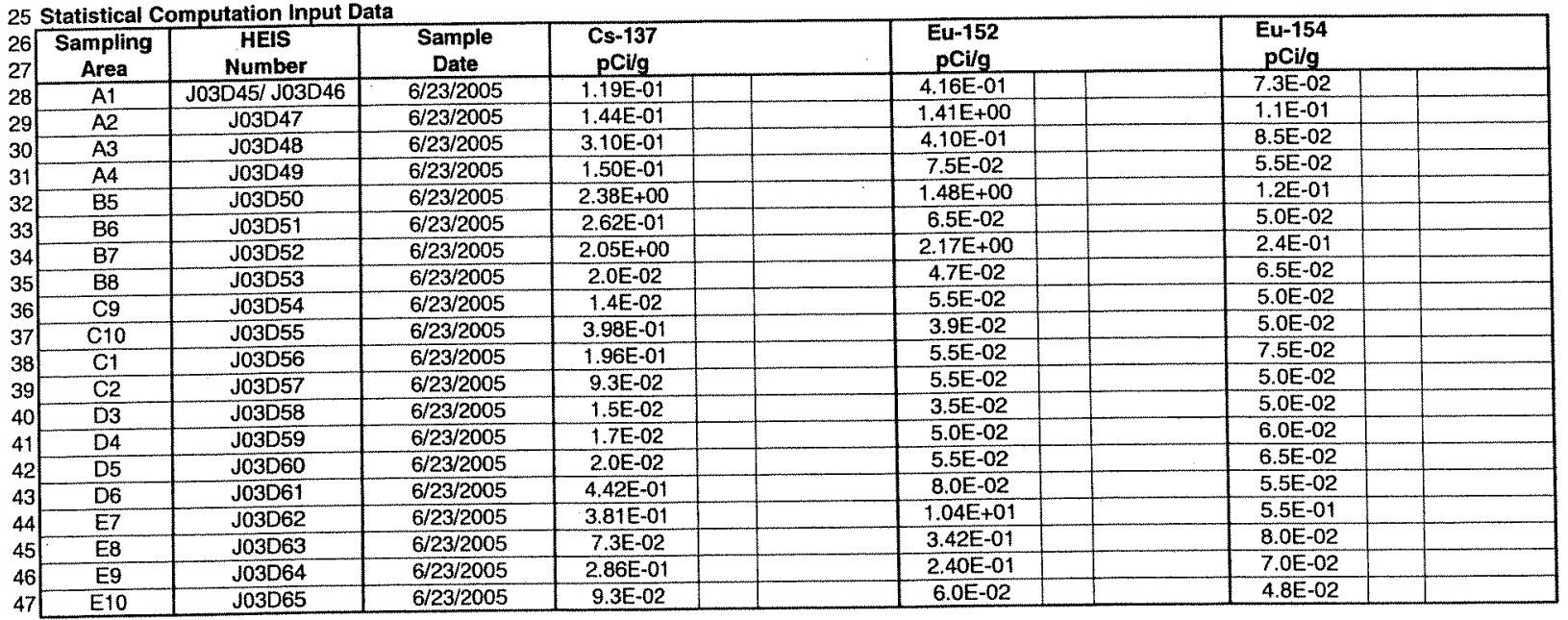

48 Statistical Computations

\begin{tabular}{|c|c|c|c|c|c|c|c|}
\hline \multirow[t]{2}{*}{49} & \multirow{3}{*}{ Statistical value based on } & \multirow{2}{*}{\multicolumn{2}{|c|}{$\begin{array}{l}\text { Cs-137 } \\
\text { Radionuclide data set. Use } \\
\text { nonparametric } z \text {-statistic. }\end{array}$}} & \multirow{2}{*}{\multicolumn{2}{|c|}{$\begin{array}{l}\text { Eu-152 } \\
\text { Radionuclide data set. Use } \\
\text { nonparametric } z \text {-statistic. }\end{array}$}} & \multirow{2}{*}{\multicolumn{2}{|c|}{$\begin{array}{l}\text { Eu-154 } \\
\text { Padionuclide data set. Use } \\
\text { nonparametric } z \text {-statistic. }\end{array}$}} \\
\hline & & & & & & & \\
\hline 51 & & 20 & & 20 & & 20 & \\
\hline 52 & $\%<$ Detection limit & $25 \%$ & & $60 \%$ & & $100 \%$ & \\
\hline $\begin{array}{r}53 \\
54\end{array}$ & mean & $3.7 \mathrm{E}-01$ & & 9.E-01 & & $1.0 \mathrm{E}-01$ & \\
\hline 54 & st. dev & $6.5 \mathrm{E}-01$ & & $2.3 \mathrm{E}+00$ & & $1.1 \mathrm{E}-01$ & \\
\hline $\begin{array}{r}55 \\
56\end{array}$ & Z-statistic & 1.645 & & 1.645 & & 1.645 & \\
\hline 56 & $95 \%$ UCL on mean & $6.1 \mathrm{E}-01$ & & $1.7 \mathrm{E}+00$ & & $1.4 \mathrm{E}-01$ & \\
\hline $\begin{array}{l}57 \\
58\end{array}$ & max value & $2.38 \mathrm{E}+00$ & & $1.04 E+01$ & & $1.1 \mathrm{E}+00$ & \\
\hline $\begin{array}{r}58 \\
59\end{array}$ & Statistical value & $6.1 \mathrm{E}-01$ & & $1.7 \mathrm{E}+00$ & & $1.4 \mathrm{E}-01$ & \\
\hline $\begin{array}{l}59 \\
60\end{array}$ & Background & NA & & NA & & NA & \\
\hline 60 & Statistical value above background & $6.1 \mathrm{E}-01$ & & $1.7 \mathrm{E}+00$ & & $1.4 \mathrm{E}-01$ & \\
\hline & Lookup Value - 15 mrern/yr for rad & 6.2 & & 3.3 & & 3.05 & \\
\hline
\end{tabular}

62 HEIS = Hanford Environmental Information System

63 MDA = minimum detectable activity

$64 \mathrm{NA}=$ not applicable

$65 Q=$ qualifier

$66 \mathrm{U}=$ undetected 
CVP-2005-00006

Rev. 0

\section{CALCULATION SHEET}

\section{Bechtel Hanford, lnc.}

Project 100-K Area Remedial Action

Subject 100-K-56:1 Pipeline Cleanup Verification 95\% UCL Calculation
Originator J.M. Capron gan C

Date 7/21/05

Job No. 22192

Zone Sample Data

\begin{tabular}{|c|c|c|c|c|c|c|c|c|c|c|c|}
\hline \multirow{2}{*}{$\begin{array}{c}\text { Sampling } \\
\text { Area }\end{array}$} & \multirow{2}{*}{$\begin{array}{c}\text { HEIS } \\
\text { Number }\end{array}$} & \multirow{2}{*}{$\begin{array}{c}\text { Sample } \\
\text { Date }\end{array}$} & \multicolumn{3}{|c|}{ Cesium-137 } & \multicolumn{3}{|c|}{ Europium-152 } & \multicolumn{3}{|c|}{ Europium-154 } \\
\hline & & & $\mathrm{pCi} / \mathrm{g}$ & $Q$ & MDA & $\mathrm{pCi} / \mathrm{g}$ & $\mathrm{Q}$ & MDA & $\mathrm{pCi} / \mathrm{g}$ & $\mathbf{Q}$ & MDA \\
\hline $\mathrm{A} 1$ & J037K9 & $6 / 15 / 2005$ & $4.57 \mathrm{E}+00$ & & $1.0 \mathrm{E}-01$ & $3.14 \mathrm{E}+01$ & & $3.5 \mathrm{E}-01$ & $4.08 \mathrm{E}+00$ & & $3.1 \mathrm{E}-01$ \\
\hline $\mathrm{A} 2$ & J037LO & $6 / 15 / 2005$ & $2.83 E+00$ & & $8.5 \mathrm{E}-02$ & $1.02 E+01$ & & $1.5 \mathrm{E}-01$ & $1.42 E+00$ & & $1.4 \mathrm{E}-01$ \\
\hline $\mathrm{A3}$ & J037L1 & $6 / 15 / 2005$ & $2.16 E+01$ & & $1.2 \mathrm{E}-01$ & $8.83 \mathrm{E}+00$ & & $3.7 \mathrm{E}-01$ & $1.18 \mathrm{E}+00$ & & $2.3 \mathrm{E}-01$ \\
\hline B4 & J037L2 & $6 / 15 / 2005$ & $5.40 \mathrm{E}+00$ & & $9.2 \mathrm{E}-02$ & $5.23 \mathrm{E}+00$ & & 1.5E-01 & $5.68 \mathrm{E}-01$ & & $1.7 \mathrm{E}-01$ \\
\hline B5 & $\mathrm{J} 037 \mathrm{~L} 3$ & $6 / 15 / 2005$ & $2.86 \mathrm{E}+01$ & & $1.1 \mathrm{E}-01$ & $1.04 \mathrm{E}+01$ & & $2.4 \mathrm{E}-01$ & $1.78 E+00$ & & 1.7E-01 \\
\hline B6 & J037L4 & $6 / 16 / 2005$ & 8.49E-01 & & $3.6 \mathrm{E}-02$ & $5.06 \mathrm{E}-01$ & & $9.4 \mathrm{E}-02$ & $1.4 \mathrm{E}-01$ & $U$ & 1.4E-01 \\
\hline C7 & J037L5 & $6 / 16 / 2005$ & $1.15 \mathrm{E}-01$ & & $4.9 \mathrm{E}-02$ & $1.0 \mathrm{E}-01$ & $U$ & $1.0 \mathrm{E}-01$ & $1.3 \mathrm{E}-01$ & $U$ & $1.3 \mathrm{E}-01$ \\
\hline $\mathrm{CB}$ & J037L6 & $6 / 16 / 2005$ & $3.28 \mathrm{E}+00$ & & $8.6 \mathrm{E}-02$ & $8.48 E+00$ & & $1.6 \mathrm{E}-01$ & $1.31 \mathrm{E}+00$ & & $1.9 \mathrm{E}-01$ \\
\hline C9 & J037L7 & $6 / 16 / 2005$ & $9.24 \mathrm{E}-01$ & & 5.1E-02 & $1.55 \mathrm{E}+00$ & & $1.2 \mathrm{E}-01$ & $1.8 \mathrm{E}-01$ & $U$ & $1.8 \mathrm{E}-01$ \\
\hline Duplicate of & J037L8 & $6 / 16 / 2005$ & 4.5E-02 & $\mathrm{u}$ & $4.5 E-02$ & 1.2E-01 & u & $1.2 \mathrm{E}-01$ & $1.6 \mathrm{E}-01$ & U & 1.6E-01 \\
\hline
\end{tabular}

14 Statistical Computation Input Data

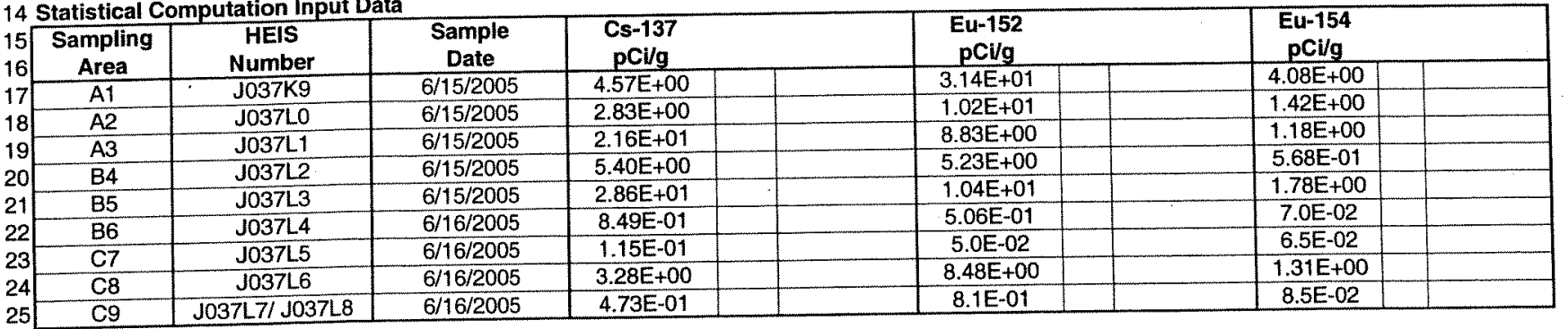

26 Statistical Computations

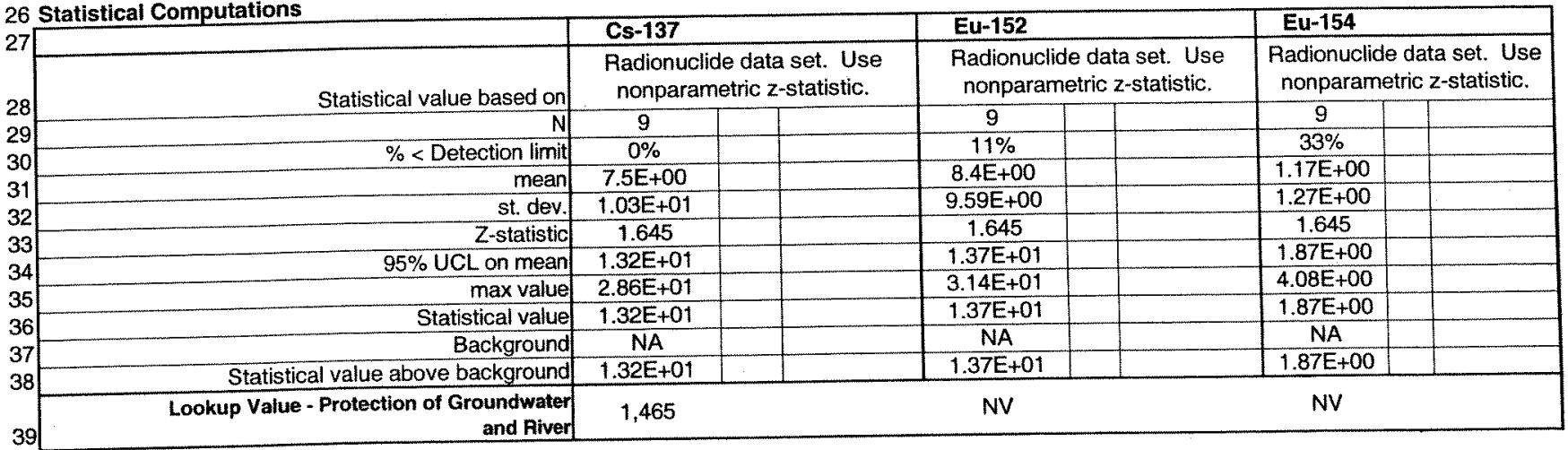

40 HEIS = Hanford Environmental Information System

$41 \mathrm{MDA}=$ minimum detectable activity

$42 \mathrm{NA}=$ not applicable

$43 \mathrm{NV}=$ no value

$44 \mathrm{Q}=$ qualifier

$45 \mathrm{U}=$ undetected 


\section{CALCULATION SHEET}

\section{Bechtel Hanford, Inc.}

Originator J.M. Capron $\operatorname{Cu}_{\mathrm{C}} \mathrm{C}$ Project 100-K Area Remedial Action Subject 100-K-56:1 Pipeline Cleanup Verification 95\% UCL Calculation
Date $7 / 21 / 05$

Job No. 22192
Calc. No. 0100K-CA-V0049 Checked T.M. Blaklex $2 \mathrm{mB}$ Checked T. B. Miley JBM
Rev. No. 0 Date $7 / 2,105$

Date $\frac{712}{1-22-05}$

Split-Duplicate Analysis

1 Shallow Zone Sample Results:

\begin{tabular}{|c|c|c|c|c|c|c|c|c|c|c|}
\hline \multirow{2}{*}{$\begin{array}{c}\text { Sampling } \\
\text { Area }\end{array}$} & \multirow[b]{2}{*}{ HEIS Number } & \multicolumn{3}{|c|}{ Cesium-137 } & \multicolumn{3}{|c|}{ Europium-152 } & \multicolumn{3}{|c|}{ Europium-154 } \\
\hline & & $\mathrm{pCi} / \mathrm{g}$ & $\mathbf{Q}$ & MDA & $\mathrm{pCi} / \mathrm{g}$ & Q & MDA & $\mathrm{pCi} / \mathrm{g}$ & $\mathbf{a}$ & MDA \\
\hline A1 & $\mathrm{J} 03 \mathrm{D} 45$ & $1.21 \mathrm{E}-01$ & & $4.6 E-02$ & $3.42 \mathrm{E}-01$ & & $7.0 \mathrm{E}-02$ & $1.3 \mathrm{E}-01$ & $\bar{U}$ & $1.3 \mathrm{E}-01$ \\
\hline Duplicate of J03D 45 & J03D46 & 1.17E-01 & & $4.8 \mathrm{E}-02$ & $4.89 \mathrm{E}-01$ & & $8.9 \mathrm{E}-02$ & $1.6 \mathrm{E}-01$ & $U$ & $1.6 \mathrm{E}-01$ \\
\hline Split of J03D45 & J03D86 & $1.51 E-01$ & & $1.52 E-02$ & 4.68E-01 & & $3.76 \mathrm{E}-02$ & $3.48 \mathrm{E}-02$ & $U$ & $5.03 \mathrm{E}-02$ \\
\hline
\end{tabular}

7 Shallow Zone Analysis:

\begin{tabular}{|c|c|c|c|c|}
\hline \multicolumn{2}{|c|}{ TDL } & 0.05 & 0.1 & 0.1 \\
\hline \multirow{3}{*}{ Duplicate Analysis } & Both > MDA? & Yes (continue) & Yes (continue) & No-Stop (acceptable) \\
\hline & Both $>5 \times$ TDL? & No-Stop (acceptable) & No-Stop (acceptable) & \\
\hline & $\mathrm{RPD}$ & & & \\
\hline \multirow{3}{*}{ Split Analysis } & Both > MDA? & Yes (continue) & Yes (continue) & No-Stop (acceptable) \\
\hline & Both > 5xTDL? & No-Stop (acceptable) & No-Stop (acceptable) & \\
\hline & RPD & & & \\
\hline
\end{tabular}

16

17 Deep Zone Sample Results:

\begin{tabular}{|c|c|c|c|c|c|c|c|c|c|c|c|}
\hline \multirow[t]{3}{*}{8} & \multirow{2}{*}{$\begin{array}{c}\text { Sampling } \\
\text { Area }\end{array}$} & \multirow[b]{2}{*}{ HEIS Number } & \multicolumn{3}{|c|}{ Cesium-137 } & \multicolumn{3}{|c|}{ Europium-152 } & \multicolumn{3}{|c|}{ Europium-154 } \\
\hline & & & $\mathrm{pCl} / \mathrm{g}$ & a & MDA & $\mathrm{pCl} / \mathrm{g}$ & $\mathbf{Q}$ & MDA & $\mathrm{pCl} / \mathrm{g}$ & $\mathbf{Q}$ & MDA \\
\hline & $\mathrm{C9}$ & J037L7 & $9.24 \mathrm{E}-01$ & & $5.1 \mathrm{E}-02$ & $1.55 E+00$ & & $1.2 \mathrm{E}-01$ & $1.8 \mathrm{E}-01$ & $\mathbf{U}$ & $1.8 \mathrm{E}-01$ \\
\hline & & & $4.5 \mathrm{E}-02$ & $\mathbf{U}$ & $4.5 \mathrm{E}-02$ & & $\mathrm{U}$ & $1.2 E$ & $1.6 \mathrm{E}-01$ & $\mathrm{U}$ & $1.6 \mathrm{E}-01$ \\
\hline & Split of J037L7 & J037L9 & $1.50 E+00$ & & $2.19 \mathrm{E}-02$ & $2.62 E+00$ & & $5.51 \mathrm{E}-02$ & $2.39 \mathrm{E}-01$ & $\mathrm{u}$ & $9.10 \mathrm{E}-02$ \\
\hline
\end{tabular}

23 Deep Zone Analysis:

\begin{tabular}{|c|c|c|c|c|c|}
\hline 24 & & & 0.05 & 0.1 & 0.1 \\
\hline 25 & & Both $>$ MDA? & No-Stop (acceptable) & No-Stop (acceptable) & No-Stop (acceptable) \\
\hline 26 & Duplicate Analysis & Both > 5xTDL? & & & \\
\hline 27 & & RPD & & & \\
\hline 28 & & Both > MDA? & Yes (continue) & Yes (continue) & No-Stop (acceptable) \\
\hline 29 & Split Analysis & Both > 5xTDL? & Yes (calc RPD) & Yes (calc RPD) & \\
\hline 30 & & $\mathrm{RPD}$ & $47.52 \%$ & $51.32 \%$ & \\
\hline
\end{tabular}

31 HEIS = Hanford Environmental Information System

32 MDA $=$ minimum detectable activity

$33 Q=$ qualifier

$34 \mathrm{RPD}=$ relative percent difference

$35 \mathrm{TDL}=$ target detection limit

$36 \mathrm{U}=$ undetected 


\section{CALCULATION COVER SHEET}

Project Title $100-\mathrm{K}$ Remedial Action Job No. 22192 Area 100-K Area

Discipline Environmental *Calc. No. 0100K-CA-V0050

Subject 100-K-56:1 Pipeline RESRAD Calculation Computer Program RESRAD Program No. Version 6.22

The attached calculations have been generated to document compliance with established cleanup levels. These documents should be used in conjunction with other relevant documents in the administrative record.

\section{$\begin{array}{llll}\text { Committed Calculation } \otimes & \text { Preliminary } \square & \text { Superseded } \square & \text { Voided }\end{array}$}

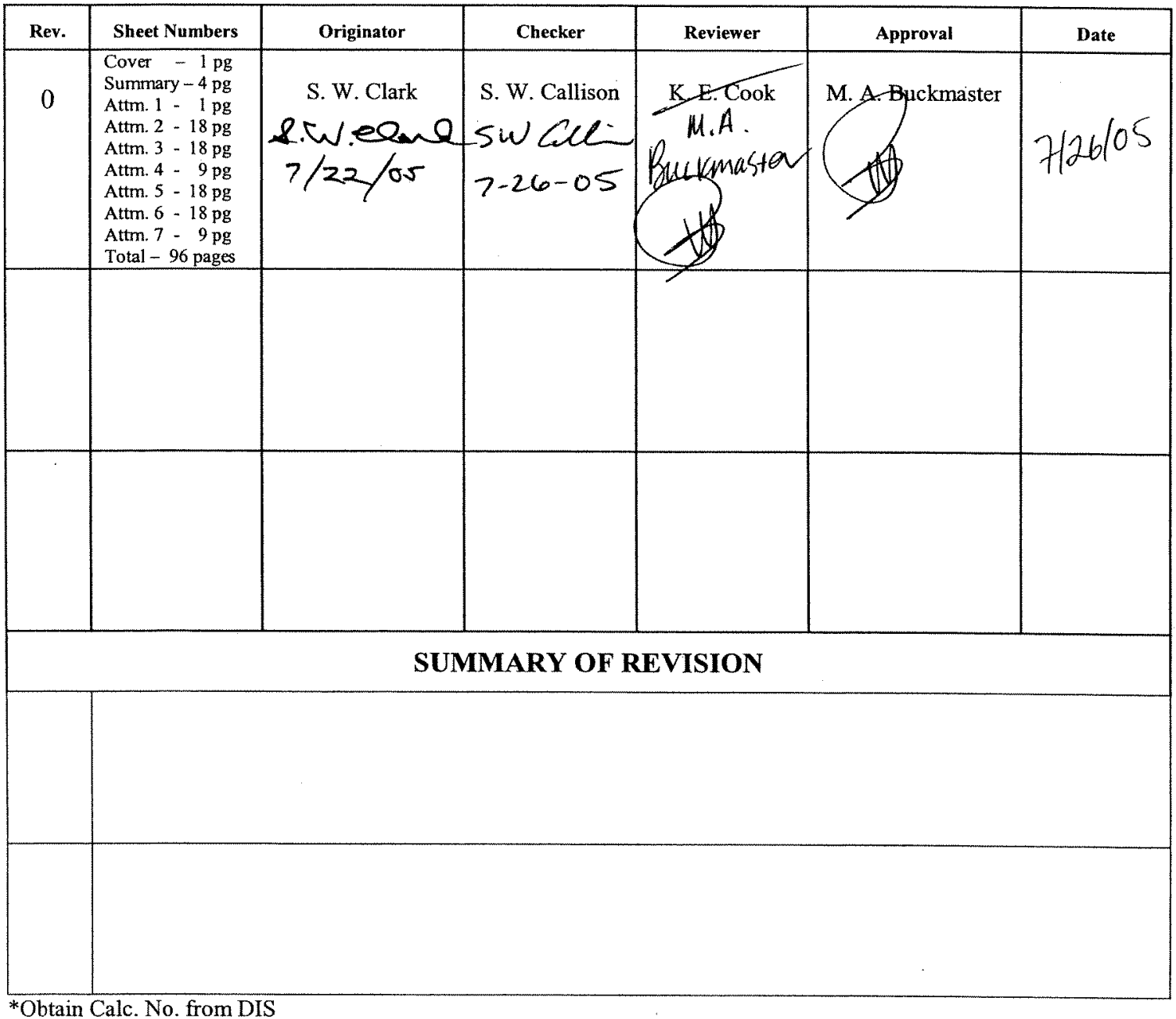


CVP-2005-00006

Rev. 0

manfored Bechtel Hanford, Inc. CALCULATION SHEET

\begin{tabular}{|r|l|l|l|l|l|l|c|}
\hline Originator: & S. W. Clark 2 V & Date: & $7 / 22 /$ as & Calc. No.: & $0100 \mathrm{~K}-\mathrm{CA}-V 0050$ & Rev.: & 0 \\
\hline Project: & $100-\mathrm{K}-56: 1$ Cleanup Verification & Job No: & 22192 & Checked: & S. W. Callison SWC & Date: & $7-26-05$ \\
\hline Subject: & $100-\mathrm{K}-56: 1$ Pipeline Cleanup Verification RESRAD Calculation & & Sheet No. 1 of 4 \\
\hline
\end{tabular}

\section{PURPOSE:}

Calculate the soil and groundwater concentrations, dose, and risk contributions from remaining radionuclide contaminants in the vadose zone over a period of 1,000 years.

\section{GIVEN/REFERENCES:}

1) Cleanup verification data from 100-K-56:1 Pipeline Cleanup Verification $95 \%$ UCL Calculation, Calculation No. 0100K-CA-V0049, Rev. 0, Bechtel Hanford, Inc., Richland, Washington.

2) Remedial Design Report/Remedial Action Work Plan for the 100 Area (RDR/RAWP), DOE/RL-96-17, Rev. 4, U.S. Department of Energy, Richland Operations Office, Richland, Washington.

3) Radioactive and nonradioactive contaminants of concern from the 100 Area Remedial Action Sampling and Analysis Plan (100 Area SAP), DOE/RL-96-22, Rev. 3, U.S. Department of Energy, Richland Operations Office, Richland, Washington. For the purpose of these RESRAD calculations, the radioactive contaminants of concern (COCs) are cesium-137, europium-152, and europium-154.

4) For the purpose of these RESRAD calculations there are no nonradionuclide contaminants of concern.

5) RESidual RADioactivity (RESRAD) computer code, version 6.22, to calculate compliance with residual radioactivity guidelines, developed for the U.S. Department of Energy by the Environmental Assessment Division of Argonne National Laboratory, Argonne, Illinois.

6) Sample design data from the 100-K-56:1 Pipeline Sample Design, Calculation No. 0100K-CA-V0053, Rev. 0, Bechtel Hanford, Inc., Richland, Washington. For the purpose of these RESRAD calculations, the sampling areas identified in the sampling plan calculation brief are $19,844 \mathrm{~m}^{2}$ for the Shallow Zone and $11,131 \mathrm{~m}^{2}$ for the Deep Zone.

\section{SOLUTION:}

1) Table 1 shows the waste site parameters used for RESRAD modeling. The model for the RESRAD runs uses the conservative assumption that the residual contamination

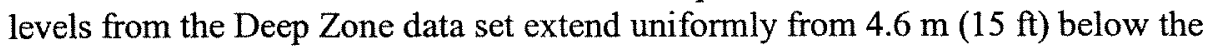
ground surface to groundwater.

2) Table 2 shows the radionuclide activities for each COC. Input factors for each RESRAD run are shown in the "Summary" section of the RESRAD "Mixture Sums and Single Radionuclide Guidelines" printouts in Attachments to this Calculation Summary. 


\begin{tabular}{|c|c|c|c|c|c|c|}
\hline Originator: & Que & Date: & ar Calc. No.: & $0100 \mathrm{~K}-\mathrm{CA}-\mathrm{V} 0050$ & Rev.: & 0 \\
\hline Project: & 100-K-56: I Cleanup Verification & Job No: & ' 22192 Checked: & S. W. Callison $\mathrm{sw} C$ & Date: & $7-26-05$ \\
\hline Subject: & \multicolumn{4}{|c|}{ 100-K-56:1 Pipeline Cleanup Verification RESRAD Calculation } & \multicolumn{2}{|c|}{ Sheet No. 2 of 4} \\
\hline
\end{tabular}

3) The year where the peak dose (or concentration) occurs from each individual radionuclide $\mathrm{COC}$ was determined by a preliminary run. This year was then included in all final RESRAD runs. For the direct exposure pathway (i.e. soil ingestion and inhalation and external radiation), the peak year occurred at year zero (year 2005) for all COCs. For the water pathways (i.e., drinking water and food ingestion) the peak year was 43 for cesium-137. The 43 -year time period was included in all RESRAD runs.

\begin{tabular}{|l|c|c|l|}
\hline \multicolumn{4}{|c|}{ Table 1. Waste Site Dimensions for RESRAD Modeling } \\
\hline \multicolumn{1}{|c|}{ Parameter } & Units & Value & \multicolumn{1}{|c|}{ Comments } \\
\hline Cover Depth & $\mathrm{m}$ & 0 & \\
\hline Area of Shallow Zone & $\mathrm{m}^{2}$ & 19,844 & Based on Sample Design $^{\text {a }}$ \\
\hline Thickness: Shallow Zone & $\mathrm{m}$ & 4.6 & Appendix B of 100 Area RDR/RAWP $^{-1}$ \\
\hline Elevation: Ground Surface & $\mathrm{m}$ & 135.5 & NAVD88 \\
\hline Elevation: Groundwater & $\mathrm{m}$ & 119.8 & NAVD88 \\
\hline Area of Deep Zone & $\mathrm{m}^{2}$ & 11,131 & Based on Sample Design $^{\text {a }}$ \\
\hline Thickness: Contaminated Deep Zone & $\mathrm{m}$ & 11.1 & Conservative assumption for modeling \\
\hline Length Parallel to Aquifer Flow & $\mathrm{m}$ & 216 & Based on Sample Design ${ }^{\text {a }}$ \\
\hline $\begin{array}{l}\text { a Sample design data from the 100-K-56:I Pipeline Sample Design, Calculation No. 0100K-CA-V0053, Rev. 0, Bechtel Hanford, } \\
\text { Inc., Richland, Washington. }\end{array}$
\end{tabular}

\section{METHODOLOGY:}

1) Runs of RESRAD version 6.22 were completed for the shallow zone using the radionuclide concentrations shown in Table 2. RESRAD numerical output reports for dose, risk, and concentration for the shallow zone are presented in the Attachments to this calculation summary.

\begin{tabular}{|c|c|c|}
\hline \multicolumn{3}{|c|}{ Table 2. Radionuclide Concentrations } \\
\hline COCs & Shallow Zone & Deep Zone \\
\hline \multicolumn{3}{|c|}{ Radionuclide Activity $(p$ Ci/g) } \\
\hline Cs-137 & 0.61 & 13.2 \\
\hline Eu-152 & 1.7 & 13.7 \\
\hline Eu-154 & 0.14 & 1.87 \\
\hline
\end{tabular}


CVP-2005-00006

Rev. 0

Bandord Bechtel Hanford, Inc.

CALCULATION SHEET

\begin{tabular}{|r|l|l|l|l|l|l|l|}
\hline Originator: & S. W. Clark \& & Date: & $7 / 28 / 05$ & Calc. No.: & $0100 \mathrm{~K}-\mathrm{CA}-\mathrm{V} 0050$ & Rev.: & 0 \\
\hline Project: & $100-\mathrm{K}-56: 1$ Cleanup Verification & Job No: & 22492 & Checked: & S. W. Callison SwC & Date: & $7-26-05$ \\
\hline Subject: & $100-\mathrm{K}-56: 1$ Pipeline Cleanup Verification RESRAD Calculation & & Sheet No. 3 of 4 \\
\hline
\end{tabular}

\section{RESULTS:}

\section{1) Radionuclide "All Pathways" Dose Rate}

The "all pathways" (maximum) dose rates are shown in Table 3. The maximum total all-pathways dose rate is $9.95 \mathrm{mrem} / \mathrm{yr}$ which occurs at year zero (2005).

\begin{tabular}{|c|c|c|c|c|c|c|c|c|c|}
\hline \multicolumn{8}{|c|}{ Table 3. All Pathway Dose Rate (mrem/yr) } \\
\hline $\begin{array}{c}\text { RESRAD } \\
\text { Run \# }\end{array}$ & $\begin{array}{c}\text { Vadose Zone } \\
\text { Horizons }\end{array}$ & \multicolumn{7}{c|}{ "All Pathways" Dose Contributions in mrem/yr at each time slice (yr) } \\
\cline { 5 - 11 } & 0 & 1 & 3 & 13 & 43 & 100 & 300 & 1000 \\
\hline 1 & Shallow Zone & $9.95 \mathrm{E}+00$ & $9.47 \mathrm{E}+00$ & $8.59 \mathrm{E}+00$ & $5.32 \mathrm{E}+00$ & $1.42 \mathrm{E}+00$ & $1.96 \mathrm{E}-01$ & $1.44 \mathrm{E}-03$ & $1.17 \mathrm{E}-10$ \\
\hline 2 & Deep Zone & $1.38 \mathrm{E}-25$ & $2.55 \mathrm{E}-03$ & $8.04 \mathrm{E}-03$ & $2.86 \mathrm{E}-02$ & $4.77 \mathrm{E}-02$ & $2.97 \mathrm{E}-02$ & $8.76 \mathrm{E}-04$ & $2.73 \mathrm{E}-10$ \\
\hline $\begin{array}{c}\text { Total All Pathways Dose } \\
\text { Rate (mrem/yr) }\end{array}$ & $9.95 \mathrm{E}+00$ & $9.47 \mathrm{E}+00$ & $8.59 \mathrm{E}+00$ & $5.34 \mathrm{E}+00$ & $1.47 \mathrm{E}+00$ & $2.25 \mathrm{E}-01$ & $2.32 \mathrm{E}-03$ & $3.90 \mathrm{E}-10$ \\
\hline
\end{tabular}

\section{2) Radionuclide Excess Cancer Risk}

The radionuclide excess lifetime cancer risk results are shown in Table 4. The maximum total excess lifetime cancer risk $\left(1.20 \times 10^{-4}\right)$ occurs at year zero (2005).

\begin{tabular}{|c|c|c|c|c|c|c|c|c|c|}
\hline \multicolumn{8}{|c|}{ Table 4. Radionuclide Excess Lifetime Cancer Risk } \\
\hline $\begin{array}{c}\text { RESRAD } \\
\text { Run \# }\end{array}$ & $\begin{array}{c}\text { Vadose Zone } \\
\text { Horizons }\end{array}$ & \multicolumn{7}{|c|}{ Excess Cancer Risk at Each Time Slice (yr) } \\
\cline { 3 - 10 } & 0 & 1 & 3 & 13 & 43 & 100 & 300 & 1000 \\
\hline 1 & Shallow Zone & $1.20 \mathrm{E}-04$ & $1.14 \mathrm{E}-04$ & $1.04 \mathrm{E}-04$ & $6.58 \mathrm{E}-05$ & $1.89 \mathrm{E}-05$ & $2.96 \mathrm{E}-06$ & $2.33 \mathrm{E}-08$ & $1.89 \mathrm{E}-15$ \\
\hline 2 & Deep Zone & $5.90 \mathrm{E}-07$ & $6.20 \mathrm{E}-07$ & $6.76 \mathrm{E}-07$ & $8.68 \mathrm{E}-07$ & $9.32 \mathrm{E}-07$ & $5.03 \mathrm{E}-07$ & $1.37 \mathrm{E}-08$ & $4.13 \mathrm{E}-15$ \\
\hline \multicolumn{2}{|c|}{ Total Excess Cancer Risk } & $1.20 \mathrm{E}-04$ & $1.15 \mathrm{E}-04$ & $1.05 \mathrm{E}-04$ & $6.66 \mathrm{E}-05$ & $1.99 \mathrm{E}-05$ & $3.46 \mathrm{E}-06$ & $3.70 \mathrm{E}-08$ & $6.03 \mathrm{E}-15$ \\
\hline
\end{tabular}

\section{3) Radionuclide Groundwater Protection}

The radionuclide concentrations in groundwater calculated by the RESRAD model are summarized in Table 5. The organ specific dose via the groundwater (and river) pathway is presented in a separate calculation brief. Only concentrations are presented here. 
CVP-2005-00006

Rev. 0

Bechtel Hanford, Inc. CALCULATION SHEET

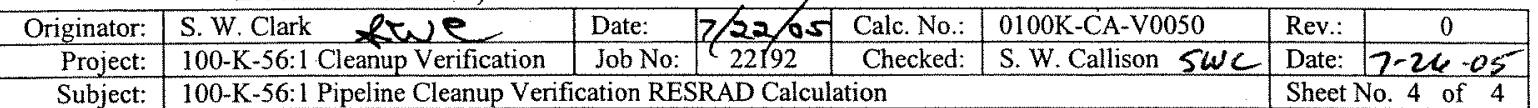

Table 5. RESRAD Predicted Radionuclide Groundwater (Well Water) Concentrations

\begin{tabular}{|c|c|c|c|c|c|c|c|c|c|c|}
\hline \multirow{2}{*}{$\begin{array}{l}\text { Radiom } \\
\text { nuclides }\end{array}$} & \multirow{2}{*}{$\begin{array}{c}\text { Vadose Zone } \\
\text { Horizon }\end{array}$} & \multicolumn{8}{|c|}{ Groundwater Concentrations in $\mathrm{pCi} / \mathrm{L}$ at Each Time Slice (yr) } & \multirow{2}{*}{$\begin{array}{c}\text { RAGs } \\
\text { From RDR }\end{array}$} \\
\hline & & 0 & 1 & 3 & 8 & 13 & 42 & 300 & 1000 & \\
\hline \multirow{3}{*}{ Cs-137 } & Shallow Zone & 0 & 0 & 0 & 0 & 0 & 0 & 0 & 0 & \multirow{3}{*}{60} \\
\hline & Deep Zone & 0 & $3.30 \mathrm{E}-02$ & $9.49 \mathrm{E}-02$ & $3.27 \mathrm{E}-01$ & $5.41 \mathrm{E}-01$ & $3.37 \mathrm{E}-01$ & $9.91 \mathrm{E}-03$ & $3.09 \mathrm{E}-09$ & \\
\hline & Total & 0 & $3.30 \mathrm{E}-02$ & $9.49 \mathrm{E}-02$ & $3.27 \mathrm{E}-01$ & $5.41 \mathrm{E}-01$ & $3.37 \mathrm{E}-01$ & $9.91 \mathrm{E}-03$ & $3.09 \mathrm{E}-09$ & \\
\hline Eu-152 & Total & 0 & 0 & 0 & 0 & 0 & 0 & 0 & 0 & 200 \\
\hline Eu-154 & Total & 0 & 0 & 0 & 0 & 0 & 0 & 0 & 0 & 60 \\
\hline
\end{tabular}

\section{CONCLUSIONS:}

- The "all pathways" (maximum) dose rates are shown in Table 3. The combined maximum all-pathways dose rate for the shallow and deep zones is $9.95 \mathrm{mrem} / \mathrm{yr}$ which occurs at year zero (2005).

- The dominant pathway for the dose rate is direct external exposure.

- The primary radionuclide contributing to the direct exposure pathway is europium-152.

- None of the site COCs are projected to exceed remedial action goals (RAGs).

- The radionuclide excess lifetime cancer risk results are shown in Table 4. The maximum combined shallow and deep zone excess lifetime cancer risk $\left(1.20 \times 10^{-4}\right)$ occurs at year zero (2005).

- Among the radionuclide contaminants of concern, cesium-137 is calculated to reach groundwater in the 1,000 years of the RESRAD model runs. Cesium-137 is predicted to reach groundwater at concentrations significantly below the RAGs.

\section{ATTACHMENTS:}

1. Graphic showing 100-K-56:1 Cleanup Verification Model (1 page)

2. RESRAD Output: 100-K-56:1 Shallow Zone Radionuclides, Mixture Sums and Single Radionuclide Guidelines (18 pages)

3. RESRAD Output: 100-K-56:1 Shallow Zone Radionuclides, Intake Quantities and Health Risk Factors (18 pages)

4. RESRAD Output: 100-K-56:1 Shallow Zone Radionuclides, Concentration of Radionuclides, ( 9 pages)

5. RESRAD Output: 100-K-56:1 Deep Zone Radionuclides, Mixture Sums and Single Radionuclide Guidelines (18 pages)

6. RESRAD Output: 100-K-56:1 Deep Zone Radionuclides, Intake Quantities and Health Risk Factors (18 pages)

7. RESRAD Output: 100-K-56:1 Deep Zone Radionuclides, Concentration of Radionuclides, ( 9 pages) 
100-K-56:1 Pipeline Cleanup Verification Model

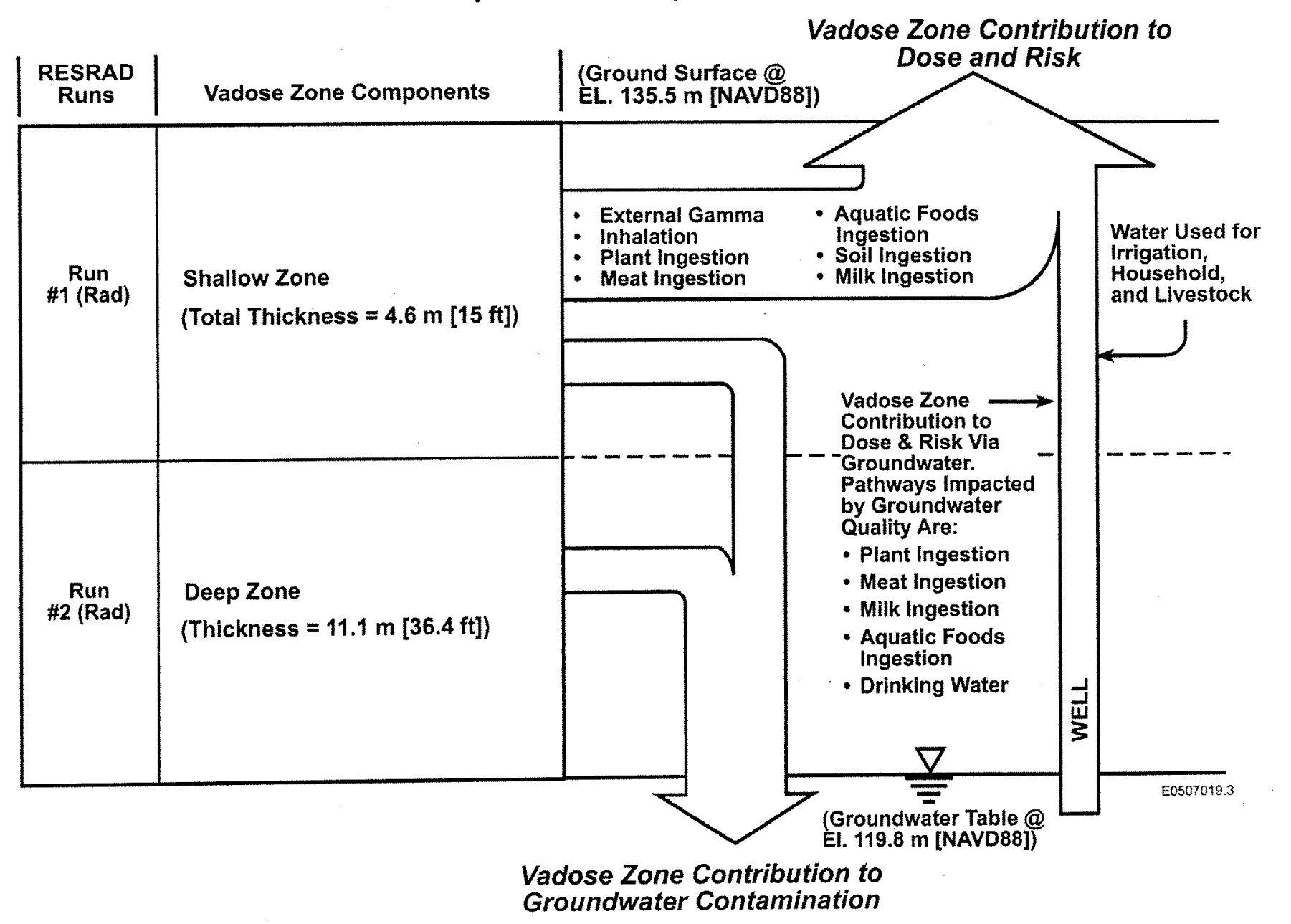




\section{CALCULATION COVER SHEET}

(Sample Design, Verification, or Waste Characterization Calculation)

\begin{tabular}{|c|c|c|c|c|}
\hline Project Title: & 100-K Remedial Action & & Job No. & 22192 \\
\hline Area & $100 \mathrm{~K}$ Area & & & \\
\hline Discipline & Environmental & *Calc. No. & $0100 \mathrm{~K}-\mathrm{CA}-\mathrm{V} 0051$ & \\
\hline Subject & 100-K-56:1 Comparison to Drinking Water Standards & (CL) Calculation & & \\
\hline Computer Program & Excel & Program No. & Excel 2003 & \\
\hline
\end{tabular}

The attached calculations have been generated to document compliance with established cleanup levels.

These documents should be used in conjunction with other relevant documents in the administrative record.

$\begin{array}{llll}\text { Committed Calculation } X & \text { Preliminary } & \text { Superseded } & \text { Voided }\end{array}$

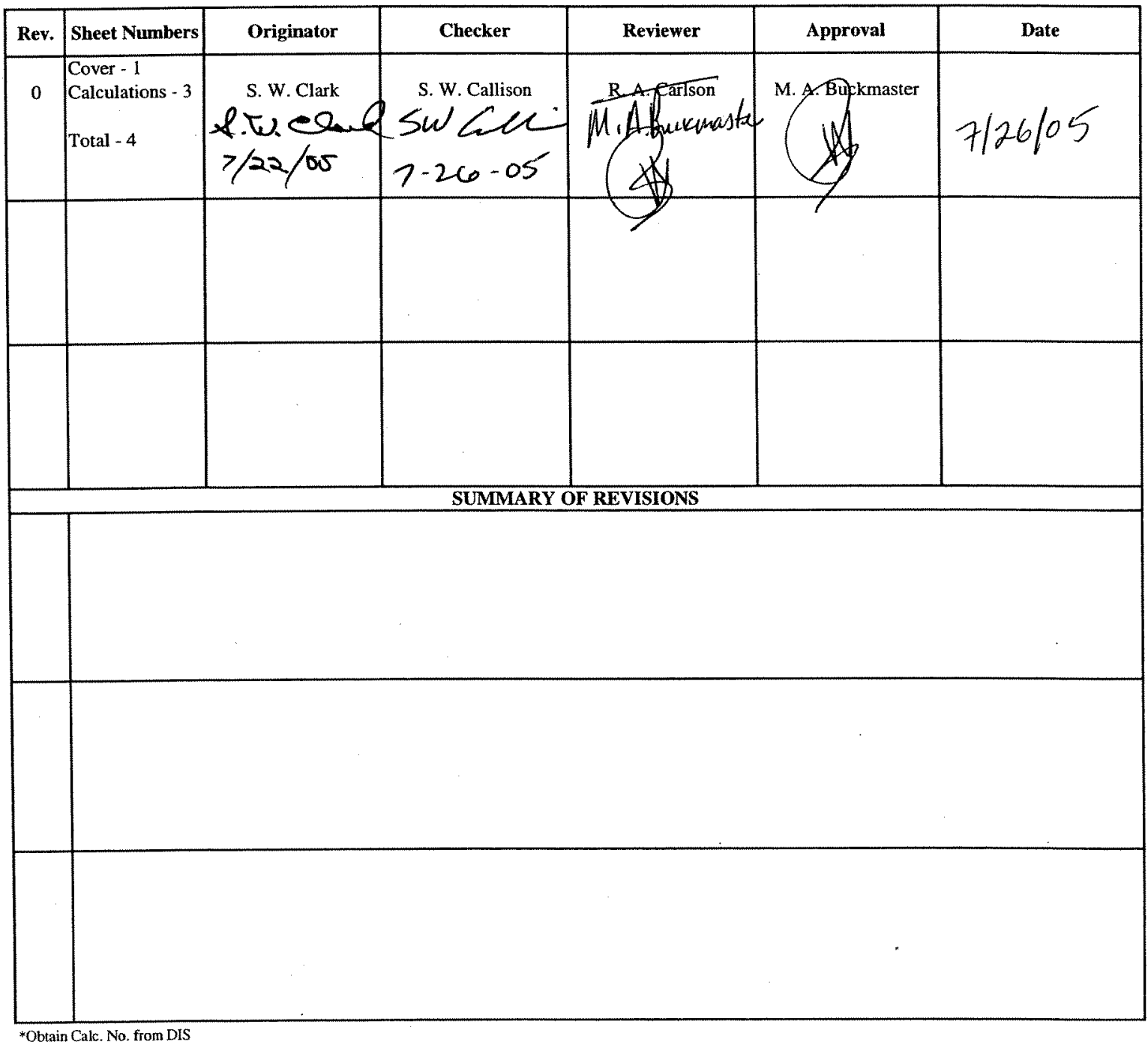


CVP-2005-00006

Rev. 0

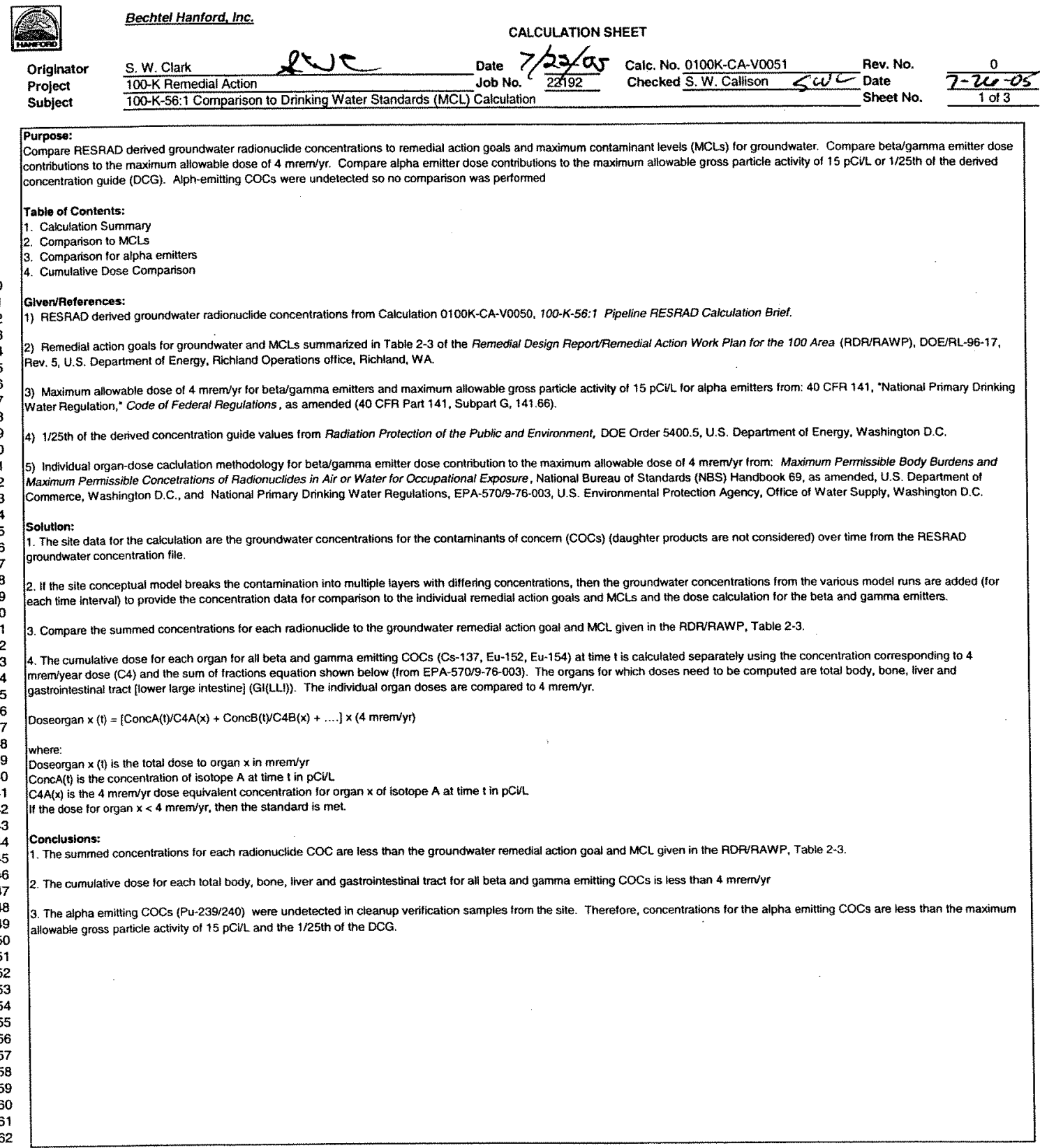




\section{CALCULATION SHEET}

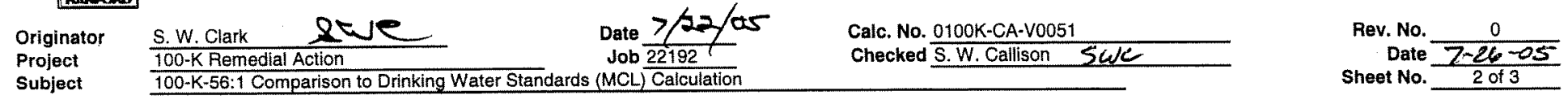

Comparison of the summed concentrations for each radionuclide to the GW MCL

Data derived from RESAAD modelling, units are $\mathrm{PCl} / \mathrm{L}$.

\begin{tabular}{|c|c|c|c|c|c|c|c|c|c|c|c|c|}
\hline Radionuclide & \multicolumn{8}{|c|}{ Time (years) } & MCL & $\begin{array}{c}\text { Exceeds } \\
\text { MCL? }\end{array}$ & Peak Conc. & $\begin{array}{c}\text { Year of } \\
\text { Peak }\end{array}$ \\
\hline Cs -137 & 0 & $3.30 \mathrm{E}-02$ & $9.49 \mathrm{E}-02$ & $3.27 \mathrm{E}-01$ & $5,41 \mathrm{E}-0 \mathrm{I}$ & $3.37 \mathrm{E}-01$ & $9.91 \mathrm{E}-03$ & $3.09 \mathrm{E}-09$ & 60 & No & $5.41 E-01$ & 43 \\
\hline Eu-152 & 0 & 0 & 0 & 0 & 0 & 0 & 0 & 0 & 200 & No & $0.00 \mathrm{E}+00$ & 0 \\
\hline & & & & & 0 & 0 & 0 & 0 & 60 & $\mathrm{No}$ & 0 OOF+00 & 0 \\
\hline Eu-154 & 0 & 0 & 0 & 0 & 0 & 0 & 0 & & $\infty 0$ & No & & \\
\hline
\end{tabular}




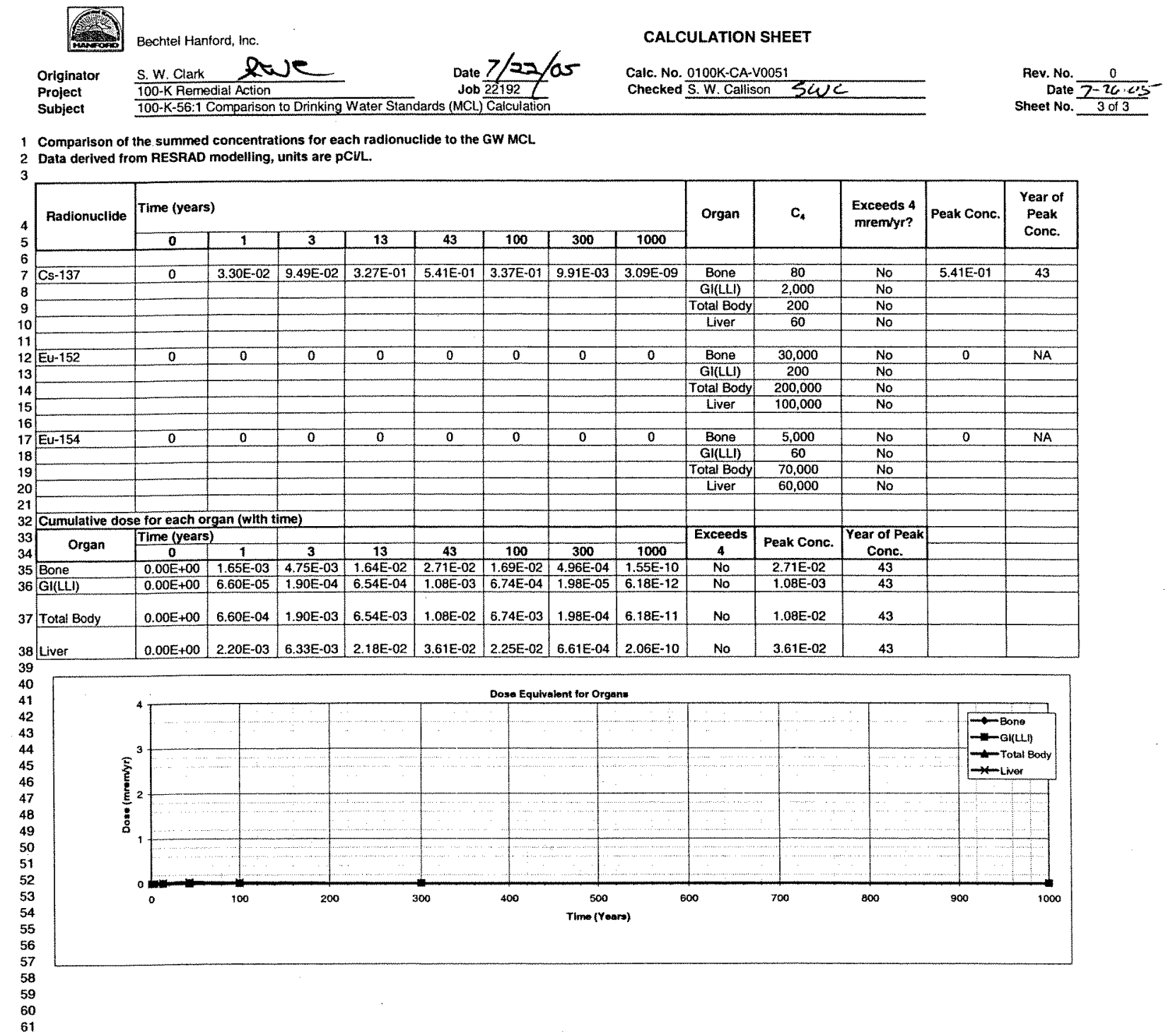




\section{CALCULATION COVER SHEET}

Project Title $100-\mathrm{K}$ Area Remedial Action

Job No. 22192

Area $100-\mathrm{K}$

Discipline Environmental

*Calc. No. 0100K-CA-V0054

Subject $116-\mathrm{KW}-4$ and 116-KE-5 Heat Recovery Stations Hazard Quotient and Carcinogenic Risk Calculations

Computer Program Excel Program No. Excel 2003

The attached calculations have been generated to document compliance with established cleanup levels. These documents should be used in conjunction with other relevant documents in the administrative record.

\section{Committed Calculation $\otimes \quad$ Preliminary $\square \quad$ Superseded $\square \quad$ Voided $\square$}

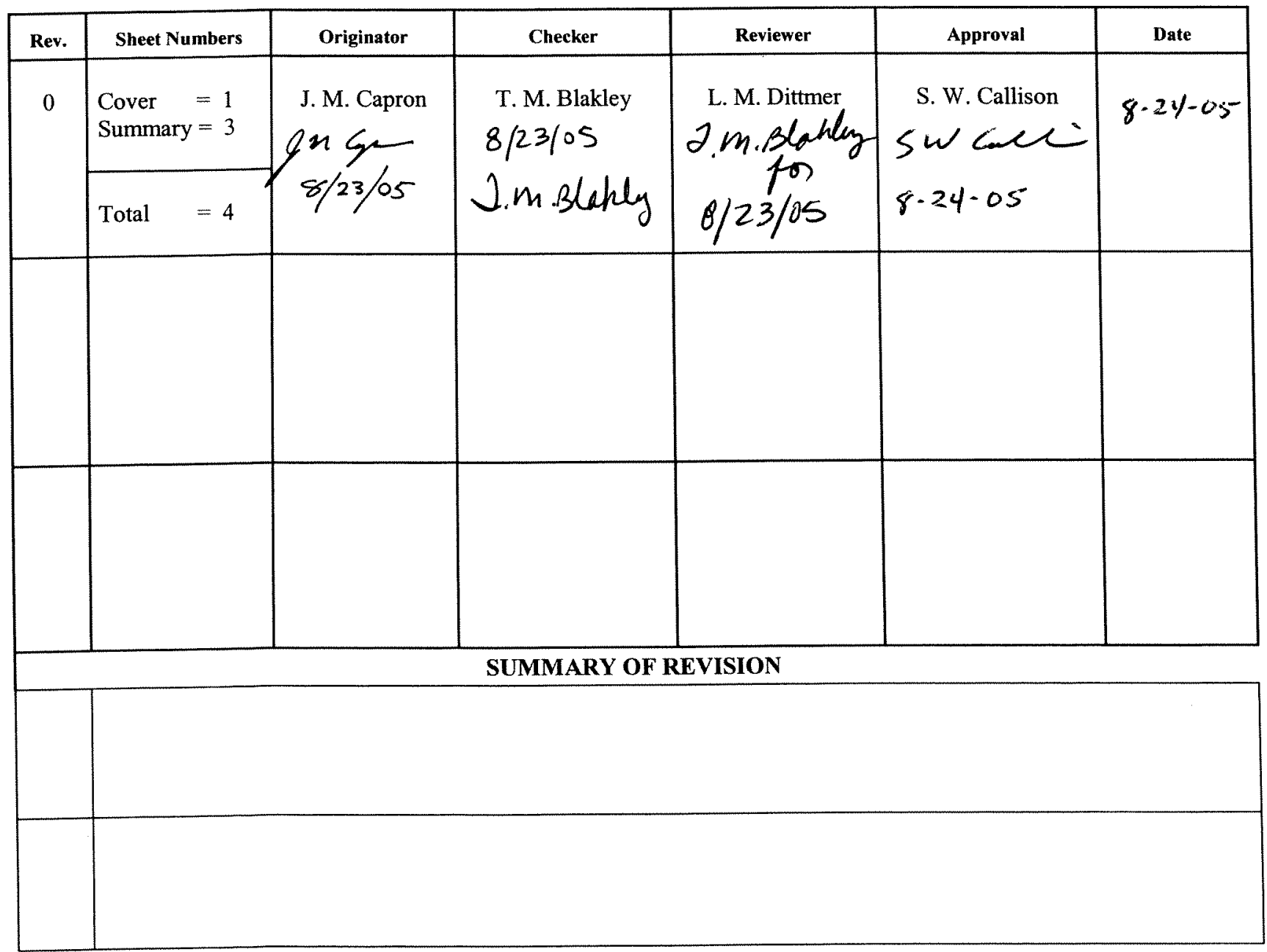

*Obtain Calc. No. from DIS 


\section{PURPOSE:}

Provide documentation to support the calculation of the hazard quotient $(\mathrm{HQ})$ and carcinogenic (excess cancer) risk values for the 116-KW-4 and 116-KE-5 Heat Recovery Stations presented in the Cleanup Verification Package for the 100-K-55:1 and 100-K-56:1 Pipelines and the 116-KW-4 and 116-KE-5 Heat Recovery Stations (BHI 2005). In accordance with the remedial action goals (RAGs) in the remedial design report/remedial action work plan (RDR/RAWP) (DOE-RL 2005), the following criteria must be met:

1) An HQ of $<1.0$ for all individual noncarcinogens

2) A cumulative $\mathrm{HQ}$ of $<1.0$ for noncarcinogens

3) An excess cancer risk of $<1 \times 10^{-6}$ for individual carcinogens

4) A cumulative excess cancer risk of $<1 \times 10^{-5}$ for carcinogens.

\section{GIVEN/REFERENCES:}

1) BHI, 2005, Cleanup Verification Package for the 100-K-55:1 and 100-K-56:1 Pipelines and the 116-KW-4 and 116-KE-5 Heat Recovery Stations, CVP-2005-00006, Bechtel Hanford, Inc., Richland, Washington.

2) DOE-RL, 2005, Remedial Design Report/Remedial Action Work Plan for the 100 Areas, DOE/RL-96-17, Rev. 5, U.S. Department of Energy, Richland Operations Office, Richland, Washington.

3) EPA, 1994, Guidance Manual for the Integrated Exposure Uptake Biokinetic Model for Lead in Children, EPA/540/R-93/081, Publication No. 9285.7-15-1, U.S. Environmental Protection Agency, Washington, D.C.

4) WAC 173-340, "Model Toxics Control Act - Cleanup," Washington Administrative Code, 1996.

\section{SOLUTION:}

1) Generate an $H Q$ for each noncarcinogenic constituent detected above background or required detection limit/practical quantitation limit and compare it to the individual HQ criterion of $<1.0$ (DOE-RL 2005).

2) Sum the HQs and compare to the cumulative $\mathrm{HQ}$ criterion of $<1.0$.

3) Generate an excess cancer risk value for each carcinogenic constituent detected above background or required detection limit/practical quantitation limit and compare it to the excess cancer risk criterion of $<1 \times 10^{-6}$ (DOE-RL 2005).

4) Sum the excess cancer risk values and compare to the cumulative cancer risk criterion of $<1 \times 10^{-5}$. 
CVP-2005-00006

Rev. 0

Harfores Bechtel Hanford, Inc. CALCULATION SHEET

Originator: J. M. Capron 2 \& C

Project: $100-\mathrm{K}$ Area Remedial Action

\begin{tabular}{r|c|c|c} 
Date: & $08 / 23 / 05$ & Calc. No.: & $0100 \mathrm{~K}-\mathrm{CA}-V 0054$ \\
Job No: & 22192 & Checked: & T. M. Blakley $h$
\end{tabular}

Subject: $\quad 116-\mathrm{KW}-4$ and 116-KE-5 Heat Recovery Stations Hazard Quotient and Carcinogenic Risk Calculations

\begin{tabular}{|c|c|c|}
\hline Rev.: & 0 \\
\hline Date: & $8 / 23 / 05$ \\
\hline & \multicolumn{2}{|c|}{ Sheet No. 2 of 3} \\
\hline
\end{tabular}

\section{METHODOLOGY:}

Hazard quotient and carcinogenic risk calculations were computed separately for the 116-KW-4 and 116-KE-5 sites using the discrete sample data from BHI (2005). Of the contaminants of potential concern for the 116-KE-5 site, chromium (total and hexavalent) and lead require the HQ and risk calculations because these analytes were detected above their Hanford Site background values. Ethylene glycol requires calculations because it was detected by laboratory analysis and cannot be attributed to natural occurrence. For the 116-KW-4 site, calculations are only required for hexavalent chromium, as no other contaminants of potential concern were detected above background levels or laboratory detection limits. An example of the HQ and risk calculations is presented below:

1) For example, the maximum value for total chromium at the $116-\mathrm{KE}-5$ site is $30 \mathrm{mg} / \mathrm{kg}$, divided by the noncarcinogenic RAG value of $120,000 \mathrm{mg} / \mathrm{kg}$ (chromium is identified as a noncarcinogen in WAC 173-340-740[3]), is $2.5 \times 10^{-4}$. Comparing this value, and all other individual values, to the requirement of $<1.0$, this criteria is met.

2) After the HQ calculation is completed for the appropriate analytes, the cumulative HQs can be obtained by summing the individual values for each site. The sums of the HQ values for the $116-\mathrm{KW}-4$ and 116-KE-5 sites are $1.0 \times 10^{-3}$ and $3.4 \times 10^{-2}$, respectively. Comparing these values to the requirement of $<1.0$, this criterion is met.

3) To calculate the excess cancer risk, the statistical value is divided by the carcinogenic RAG value, then multiplied by $1 \times 10^{-6}$. For example, the maximum value for hexavalent chromium at the $116-\mathrm{KW}-4$ site is $0.24 \mathrm{mg} / \mathrm{kg}$; divided by $2.1 \mathrm{mg} / \mathrm{kg}$ and multiplied as indicated is $1.1 \times 10^{-7}$. Comparing this value, and all other individual values, to the requirement of $<1 \times 10^{-6}$, this criterion is met.

4) After these calculations are completed for the carcinogenic analytes, the cumulative excess cancer risk can be obtained by summing the individual values. The sums of the excess cancer risk values for the $116-\mathrm{KW}-4$ and $116-\mathrm{KE}-5$ sites are $1.1 \times 10^{-7}$ and $1.6 \times 10^{-7}$, respectively. Comparing these values to the requirement of $<1 \times 10^{-5}$, this criterion is met.

\section{RESULTS:}

1) List individual noncarcinogens and corresponding HQs $>1.0$ : None

2) List the cumulative noncarcinogenic $H Q>1.0$ : None

3) List individual carcinogens and corresponding excess cancer risk $>1 \times 10^{-6}$ : None

4) List the cumulative excess cancer risk for carcinogens $>1 \times 10^{-5}:$ None.

Tables 1 and 2 show the results of the calculations for the 116-KW-4 and 116-KE-5 sites, respectively. 
Originator: J.M. Capron 43K Project: $100-\mathrm{K}$ Area Remedial Action

\begin{tabular}{|r|c|c|c|}
\hline Date: & $08 / 23 / 05$ & Calc. No.: & 0100K-CA-V0054 \\
\hline
\end{tabular} \begin{tabular}{l|c|c|c} 
Job No: & 22192 & Checked: & T. M. Blakley $\operatorname{Lm} \beta$
\end{tabular}

Subject: $116-\mathrm{KW}-4$ and 116-KE-5 Heat Recovery Stations Hazard Quotient and Carcinogenic Risk Calculations

\begin{tabular}{|c|c|c|}
\hline Rev: & 0 \\
\hline Date: & $8 / 2^{3} / 05$ \\
\hline \multicolumn{2}{|c|}{ Sheet No. 3 of 3} \\
\hline
\end{tabular}

Table 1. Hazard Quotient and Excess Cancer Risk Results for the 116-KW-4 Site.

\begin{tabular}{|l|c|c|c|c|c|}
\hline Contaminants of Potential Concern & $\begin{array}{c}\text { Maximum } \\
\text { Value } \\
(\mathbf{m g} / \mathbf{k g})\end{array}$ & $\begin{array}{c}\text { Noncarcinogen } \\
\mathbf{R A G}^{\mathbf{b}} \\
(\mathbf{m g} / \mathbf{k g})\end{array}$ & $\begin{array}{c}\text { Hazard } \\
\text { Quotient }\end{array}$ & $\begin{array}{c}\text { Carcinogen } \\
\text { RAG } \\
(\mathbf{m g} / \mathbf{k g})\end{array}$ & $\begin{array}{c}\text { Carcinogen } \\
\text { Risk }\end{array}$ \\
\hline Metals & 0.24 & 240 & $1.0 \mathrm{E}-03$ & 2.1 & $1.1 \mathrm{E}-07$ \\
\hline Chromium, hexavalent & & & & \\
\hline Totals & & $\mathbf{1 . 0 E}-\mathbf{0 3}$ & & $\mathbf{1 . 1 E - 0 7}$ \\
\hline Cumulative Hazard Quotient: & & & & \\
\hline Cumulative Excess Cancer Risk:
\end{tabular}

\section{Notes:}

$\mathrm{RAG}=$ remedial action goal

.. = not applicable

${ }^{a}=$ From Table 4 (BHI 2005).

$b=$ Value obtained from Washington Administrative Code (WAC) 173-340-740(3), Method B, 1996, unless otherwise noted.

$c=$ Value for the carcinogen RAG calculated based on the inhalation exposure pathway (WAC) 173-340-750(3), 1996.

Table 2. Hazard Quotient and Excess Cancer Risk Results for the 116-KE-5 Site.

\begin{tabular}{|c|c|c|c|c|c|}
\hline Contaminants of Potential Concern & $\begin{array}{c}\text { Maximum } \\
\text { Value }^{\mathrm{a}} \\
(\mathrm{mg} / \mathrm{kg})\end{array}$ & $\begin{array}{c}\text { Noncarcinogen } \\
\text { RAG }^{\mathbf{b}} \\
(\mathrm{mg} / \mathrm{kg})\end{array}$ & $\begin{array}{c}\text { Hazard } \\
\text { Quotient }\end{array}$ & $\begin{array}{c}\text { Carcinogen } \\
\text { RAG } \\
\text { (mg/kg) } \\
\end{array}$ & $\begin{array}{c}\text { Carcinogen } \\
\text { Risk }\end{array}$ \\
\hline \multicolumn{6}{|l|}{ Metals } \\
\hline Chromium, total & 30 & 120,000 & $2.5 \mathrm{E}-04$ & & \\
\hline Chromium, hexavalent $^{c}$ & 0.33 & 240 & $1.4 \mathrm{E}-03$ & 2.1 & $1.6 \mathrm{E}-07$ \\
\hline Lead $^{d}$ & 11.3 & 353 & $3.2 \mathrm{E}-02$ & - & -- \\
\hline \multicolumn{6}{|l|}{ Semivolatiles } \\
\hline Ethylene glycol & 59 & 160,000 & $3.7 \mathrm{E}-04$ & & \\
\hline \multicolumn{6}{|l|}{ Totals } \\
\hline \multicolumn{3}{|l|}{ Cumulative Hazard Quotient: } & $3.4 E-02$ & & \\
\hline Cumulative Excess Cancer Risk: & & & & & $1.6 \mathrm{E}-07$ \\
\hline
\end{tabular}

Notes:

RAG = remedial action goal

.- = not applicable

${ }^{a}=$ From Table 4 (BHI 2005).

$\mathrm{b}=$ Value obtained from Washington Administrative Code (WAC) 173-340-740(3), Method B, 1996, unless otherwise noted.

$\mathfrak{c}=$ Value for the carcinogen RAG calculated based on the inhalation exposure pathway (WAC) 173-340-750(3), 1996.

$d=$ Value for the noncarcinogen RAG obtained from EPA (1994).

\section{CONCLUSION:}

This calculation demonstrates that the 116-KW-4 and 116-KE-5 sites meet the requirements for the hazard quotients and carcinogenic (excess cancer) risk as identified in the RDR/RAWP (DOE-RL 2005). 


\section{DISTRIBUTION}

U.S. Department of Energy

Richland Operations Office

J. Zeisloft (5)

A3-04

DOE-RL Public Reading Room

$\mathrm{H} 2-53$

Fluor Hanford, Inc.

J. P. Shearer (WIDS)

A0-21

Washington Closure Hanford

K. A. Anselm H9-02

M. A. Buckmaster X3-16

S. W. Callison X3-16

R. A. Carlson $\quad \mathrm{XO}-17$

S. W. Clark H9-01

L. A. Dietz HO-23

L. M. Dittmer H9-01

J. E. Thomson HO-23

Records and Document Control H0-30

Hanford Technical Library P8-55 
CVP-2005-00006

Rev. 0

Distr-2 